\title{
FORMULATION OF AN OPTIMAL SEARCH STRATEGY FOR SPACE DEBRIS AT GEO
}

\author{
A Thesis \\ presented to \\ the Faculty of California Polytechnic State University, \\ San Luis Obispo
}

In Partial Fulfillment

Of the Requirement for the Degree

Master of Science in Aerospace Engineering

By

Daniel Jonathan Jackson

November 2011 
(C)2011

Daniel Jonathan Jackson

ALL RIGHTS RESERVED 


\section{Committee Membership Page}

TITLE:

AUTHOR:

DATE SUBMITTED:

COMMITTEE CHAIR:

COMMITTEE MEMBER: Dr. Ed Barker

COMMITTEE MEMBER: Dr. Eric A. Miehl

COMMITTEE MEMBER: Dr. Kristina Jameson
FORMULATION OF AN OPTIMAL SEARCH STRATEGY FOR SPACE DEBRIS AT GEO

Daniel Jonathan Jackson

November 21, 2011

Dr. Kira Abercromby 


\title{
ABSTRACT
}

\section{FORMULATION OF AN OPTIMAL SEARCH STRATEGY FOR SPACE DEBRIS AT GEO}

\author{
By \\ Daniel Jonathan Jackson
}

The purpose of this thesis is to create a search strategy to find orbital debris when the object fails to appear in the sky at its predicted location. This project is for NASA Johnson Space Center Orbital Debris Program Office through the MODEST (Michigan Orbital Debris Survey Telescope) program. This thesis will build upon the research already done by James Biehl in "Formulation of a Search Strategy for Space Debris at GEO.” MODEST tracks objects at a specific right ascension and declination. A circular orbit assumption is then used to predict the location of the object at a later time. Another telescope performs a follow-up to the original observation to provide a more accurate orbit predication. This thesis develops a search strategy when the follow-up is not successful. A general search strategy for finding space debris was developed based on previous observations. A GUI was also generated to find a search strategy in real-time for a specific object based upon previous observations of that object.

Search strategies were found by adding a $2 \%$ mean random error to the position and velocity vectors. Adding a random error allows for finding the most likely location of space debris when the orbital elements are slightly incorrect. A bivariate kernel density estimator was used to find the probability density function. The probability density function was used to find the most probable location of an object. A correlation between error in the orbital elements and error in right ascension and declination root mean square (RMS) error was investigated. It was found that the orbital elements affect the RMS error 
nonlinearly, but the relation between orbital element and error depended on the object and no general pattern was found. It was found that how long after the original object was found until the follow-up was attempted did not have a large impact on the probability density function or the search strategy. 


\section{TABLE OF CONTENTS}

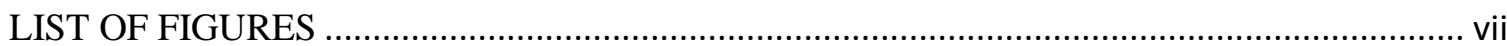

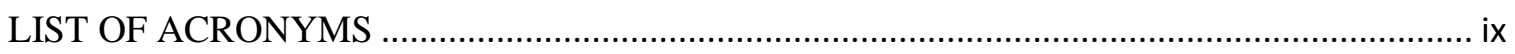

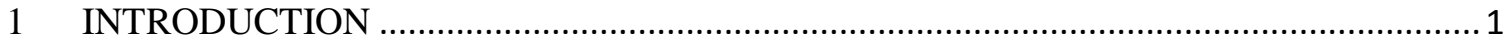

1.1 Purpose

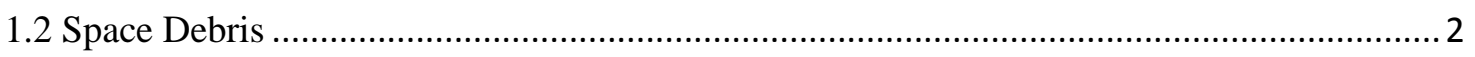

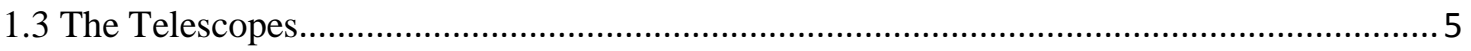

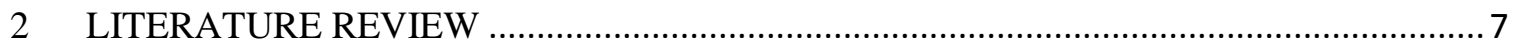

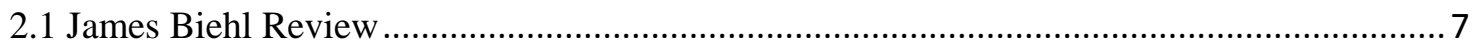

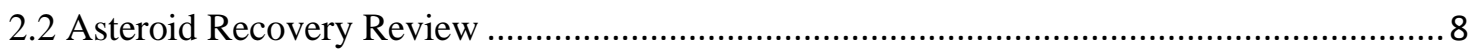

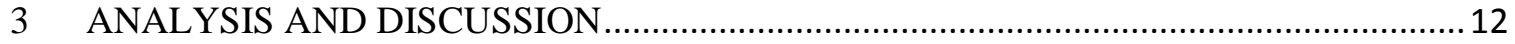

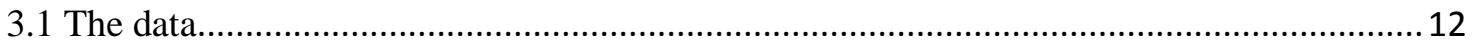

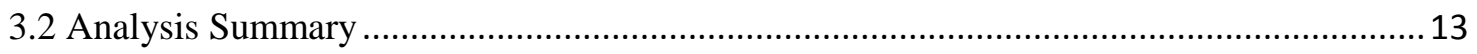

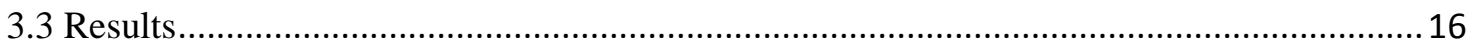

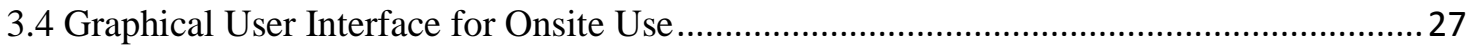

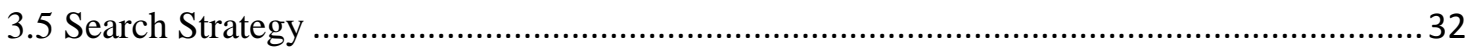

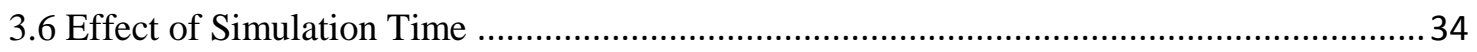

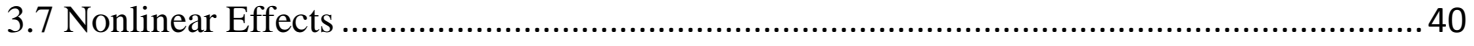

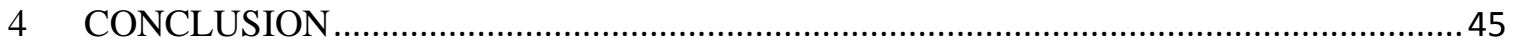

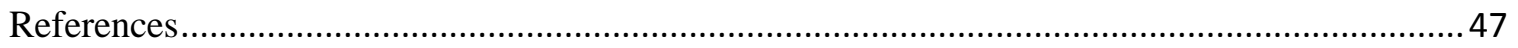

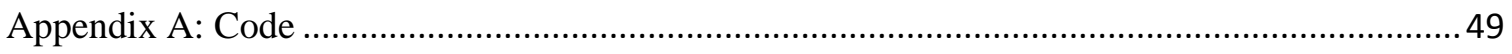

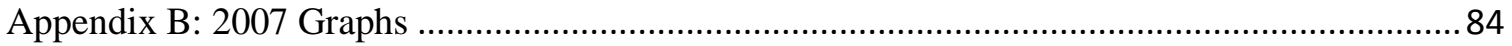

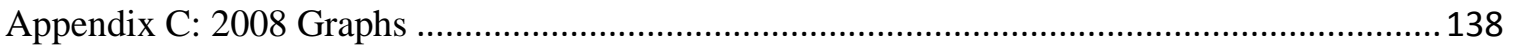

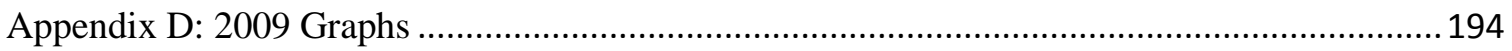




\section{LIST OF FIGURES}

Figure 1. The Cerro Tololo Inter-American Observatory with C9 on the left and

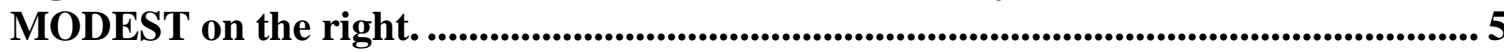

Figure 2. An example of a survey detection sequence from MODEST. Each subimage is 2.5 arc-minutes square...........................................................................................6 6

Figure 3. Zoomed in View with search grid for low INC orbits telescope limitations 8

Figure 4. Distribution of Klet' NEA recoveries in the sky. ....................................... 10

Figure 5. The simulated recovery of the asteroid 9076........................................ 11

Figure 6. Summary of the analysis. .............................................................................. 15

Figure 7. The right ascension versus declination distribution for object 70312...... 17

Figure 8. The right ascension versus declination distribution for object 70302 ...... 18

Figure 9. The right ascension versus declination distribution for object 70321...... 19

Figure 10. The probability density functions for object 70312................................... 20

Figure 11. The probability density functions for object 70302................................ 21

Figure 12. Each square represents the field of view of the telescope with the center square representing the field of view with object 70312 in the center 20 minutes after the last observation. The green square represents the predicted location of object 70312 .

Figure 13. Each square represents the field of view of the telescope with the center square representing the field of view with object 70302 in the center 20 minutes after the last observation. The green square represents the predicted location of object70302........................................................................19

Figure 14. Designations of possible areas for the telescope to move to...................... 24

Figure 15. This figure shows the number of times and percent that each region had the highest probability of finding an object for all 73 objects

Figure 16. Optimal search strategy for the telescope. 
Figure 17. Realistic search strategy given physical constraints of the telescope.

Figure 18. Start up screen of the executable.............................................................. 28

Figure 19. Browser to navigate and select the TLE............................................ 29

Figure 20. The load bar shows the progress and what iteration the program is on. 30

Figure 21. The probability density function displayed in the GUI. .......................... 31

Figure 22. An alternative search strategy for object 70304................................... 33

Figure 23. The right ascension versus declination distribution for object 70312 for 20 minutes of simulation time.................................................. 35

Figure 24. The right ascension versus declination distribution for object 70312 for

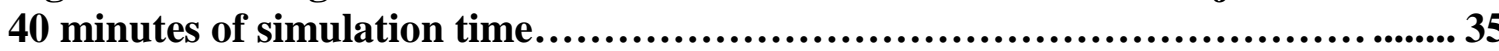

Figure 25. The right ascension versus declination distribution for object 70312 for 60 minutes of simulation time.................................................... 36

Figure 26. Field of view of the telescope with the center square representing the field of view with object 70312 in the center 20 minutes after the last observation............

Figure 27. Field of view of the telescope with the center square representing the field of view with object 70312 in the center 40 minutes after the last observation...........

Figure 28. Field of view of the telescope with the center square representing the field of view with object 70312 in the center 60 minutes after the last observation...............

Figure 29. Right ascension versus declination when the error is added to the orbital elements........................................................................... 40

Figure 30. The statistical analysis of object 70304 from MINITAB......................... 43 


\section{LIST OF ACRONYMS}

\begin{tabular}{|c|c|}
\hline AP & Argument of Perigee \\
\hline C9 & Smarts 0.9 m Telescope \\
\hline $\mathrm{COE}$ & Classical Orbital Elements \\
\hline CTIO & Cerro Tololo Inter-American Observatory \\
\hline CCD & Charge-Coupled Device \\
\hline DEC & Declination \\
\hline ECC & Eccentricity \\
\hline GEO & Geosynchronous Orbit \\
\hline GUI & Graphical User Interface \\
\hline HEO & high Earth orbit \\
\hline HST & Hubble Space Telescope \\
\hline INC & Inclination \\
\hline ISS & International Space Station \\
\hline JSC & Johnson Space Center \\
\hline
\end{tabular}


LEO low Earth orbit

MANOM Mean Anomaly

MODEST Michigan Orbital Debris Survey Telescope

NASA National Aeronautics and Space Administration

NEA Near Earth Asteroid

RA Right Ascension

RMS Root Mean Square

RAAN Right Ascension of the Ascending Node

SEDS-2 Small Expendable Deployer System-2

TLE Two Line Element 


\section{INTRODUCTION}

\subsection{Purpose}

The purpose of this thesis is to create a search strategy to reacquire orbital debris when the object fails to appear in the sky at the predicted location. This is for NASA Johnson Space Center Orbital Debris Program Office through the MODEST (Michigan Orbital Debris Survey Telescope) program. The MODEST telescope is used to locate objects by tracking specific right ascension, declination, and time. A circular orbit prediction is used to predict the future orbit because the first observation does not contain enough data to accurately predict the eccentricity. Using a circular orbit assumption, the orbit of the object is then predicted. The 0.9 meter telescope at CTIO is known as C9.The orbit is used find the right ascension and declination at a later time, and C9 is used to relocate the object. The data from observing the object with $\mathrm{C} 9$ is then used to find a more accurate orbit. The $\mathrm{C} 9$ telescope will track the object several times, sometimes over several nights. Sometimes when the C9 goes on a "follow-up" to relocate the object, the object is not found.

The goal is to develop a search strategy that will increase the likelihood of finding an object that is not found in its predicted location. This thesis will build upon the research already done on this by James Biehl in "Formulation of a Search Strategy for Space Debris at GEO." This thesis will also investigate the correlation between errors in the orbital elements to the RMS location error. The orbital elements used in this report are Inclination, Eccentricity, Argument of Perigee, Right Ascension of the Ascending Node, Mean Anomaly, and Mean Motion. 


\subsection{Space Debris}

Space debris is defined as a manmade object orbiting earth that does not have a useful purpose. ${ }^{2}$ Examples of space debris are upper stages of launch vehicles, debris released during spacecraft separation or mission operations, and flecks of paint released from thermal stress. Most debris is within 2,000 km of the Earth's surface with the highest concentration at $800-850 \mathrm{~km} .^{3}$ The average impact speed of orbital debris is 10 $\mathrm{km} / \mathrm{s}$. Debris with an altitude less than $600 \mathrm{~km}$ will usually fall into the atmosphere within several years, while debris with altitudes above $1,000 \mathrm{~km}$ will usually orbit Earth for over a century. ${ }^{3}$ The exact time before entering the atmosphere depends on the altitude, eccentricity, and drag. Ground-based space debris measurements can be done by radar or optically.

Radar measurements are usually used in low Earth orbit (LEO), while optical measurements are used for high Earth orbit (HEO). Radar is well suited for ground-based observations because of its all-weather and day-and-night performance. The power budget, operating wavelengths, and the size and orientation of the object are the limiting factors for detection of small objects at long ranges using radar. From a radar measurement, the orbital elements, size and shape, orbital lifetime, ballistic coefficient, mass, and material properties can be determined.

For optical measurements, debris can be detected by a telescope when the debris object is sunlit and the sky background is dark. Objects in LEO can only be observed for a few hours, just after sunset or before sunrise. For objects in HEO and GEO, observations can be continued for the entire night. Optical measurements are limited by the requirement of clear and dark skies, and the size and orientation of the object. 
Space-based measurements have a smaller distance between the observer and the object and thus have a higher resolution. There are also no disturbances from the atmosphere. The cost of space-based measurements is usually much higher than the ground-based observations. The complexity of designing and maintaining a satellite drives the cost very high. Cost versus performance trade-off studies must be done carefully before space-based space debris monitoring can be started.

Large space debris are defined as objects greater than $10 \mathrm{~cm} .{ }^{2}$ In mid-2009, NASA estimated there were 19,000 space debris larger than $10 \mathrm{~cm}, 500,000$ space debris with a diameter between $1 \mathrm{~cm}$ and $10 \mathrm{~cm}$, and tens of millions of space debris less than $1 \mathrm{~cm} .{ }^{3}$ Satellite explosions and collisions are the primary source of large debris. The intentional destruction of the Chinese Fengyun-1C weather satellite in 2007 and the collision of an American and Russian satellite in 2009 significantly increased the amount of large debris in space. Large space debris can be tracked, and orbital elements are maintained as accurately as possible. Collisions between functional satellites and space debris are not common because the probability is so low. As the number of functional satellites and large space debris increases, the risk for destructive collisions will increase. Collisions between large space debris will also increase the small space debris population. The number of space debris less than 1 meter in diameter near GEO is not well known. Small space debris with a diameter of a few millimeters has caused damage to operations space systems. Small space debris has caused damage to shuttle windows, damage to HST (Hubble Space Telescope) high gain antenna, severed the SEDS-2 (Small Expendable Deployer System-2) tether, and damaged other exposed shuttle surfaces. 
Space debris models are mathematical descriptions of the distribution of objects in space, the movement, and the physical characteristics of the objects. These models must account for the increase in the space debris population over time. New launches add launch vehicle upper stages, payloads, and mission related objects. On-orbit maneuvers that use solid rocket motors adds space debris. Break-ups by explosions and collisions as well as material separation from surfaces increase the population. Material leakage such as nuclear power source coolant is another factor. About 12 percent of the catalogued space debris consist of objects discarded during normal satellite deployment and operations. ${ }^{2}$ These models have a small amount of data to validate the derived relationships. Accurately modeling the long term space debris population requires information on future space flight activities. Since future space plans are tentative, always changing, and often behind schedule, this is very difficult.

Studies have shown that the space debris growth can be limited by reducing the orbital lifetimes. Reducing orbital lifetimes can be achieved with controlled re-entry or lowering the altitude of the orbit. Shields can be effective for spacecraft subjected to small particles. Protection against particles less than $1 \mathrm{~cm}$ can be achieved by shielding. ${ }^{2}$ The International Space Station (ISS) has the most shields of any spacecraft. The ISS shields can withstand collisions with space debris of $1 \mathrm{~cm}$. Collision avoidance is difficult and impacts satellite operations. Collision avoidance uses propellant and can interrupt payload activities. Collision avoidance is most successful when the uncertainty in the approach distance is small. The acceptable risk criterion of NASA is 1 in $100,000 .^{2}$ 


\subsection{The Telescopes}

The University of Michigan and NASA have been using MODEST since early 2001. In March of 2005, the modernization of the telescope allowed for observation modes other than tracking at the sidereal rate, and allowed for more accurate orbits for all types of objects. In March of 2007, observations with two telescopes began. It has been used to observe orbital debris with a geostationary orbit (GEO). The goal is to characterize a complete sample of faint GEO objects selected on the basis of angular rates and brightness. The two telescopes used are the MODEST survey telescope and the CTIO 0.9-m telescope for follow-up which can be seen in Figure 1 below.

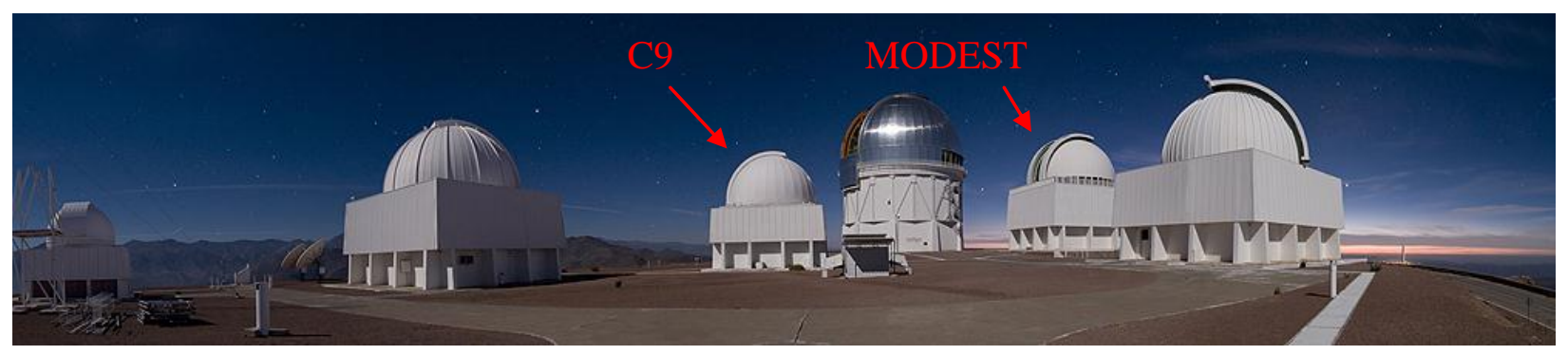

Figure 1. The Cerro Tololo Inter-American Observatory with C9 on the left and MODEST on the right.

MODEST is a 0.61/0.91-m Schmidt telescope that is located near La Serena, Chile at the

Cerro Tololo Inter-American Observatory (CTIO). The camera is a charge coupled device (CCD) with a resolution of 2048 x 2048 pixels. It has a field-of-view of 1.3 degrees by 1.3 degrees and 2.318 arc-seconds per pixel. In a 5 second exposure the maximum magnitude is $\mathrm{R}=18^{\text {th }}$ with a signal to noise ratio of 10 . In survey mode, MODEST tracks at the sidereal rate so that the right ascension (RA) and declination (DEC) are constant and point close to the anti-solar point and outside of Earth's shadow. The GEO objects appear as short streaks and stars appear as fixed length streaks. Figure 2 
below shows a sample detection. Most observations are for 5.3 minutes and 8 independent positions can be measured. For a real detection 4 independent positions are required.
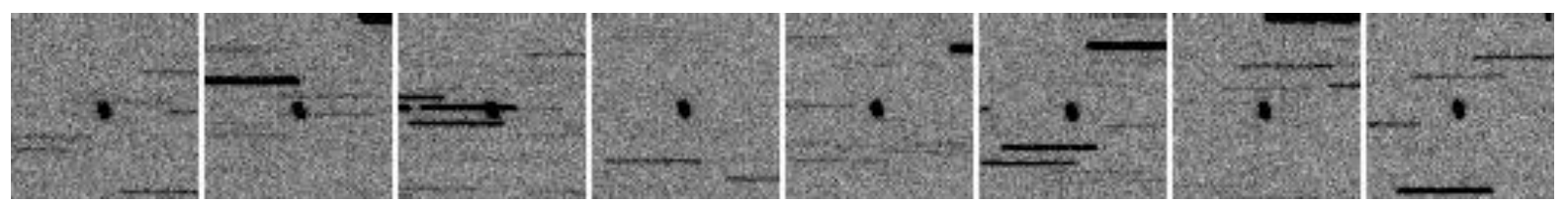

Figure 2. An example of a survey detection sequence from MODEST. Each sub-image is 2.5 arc-minutes square. ${ }^{4}$

The follow-up telescope is the CTIO 0.9-m Cassegrain telescope with a blue sensitive CCD. It has a field-of-view of 0.22 degrees by 0.22 degrees and 0.792 arc-seconds per pixel. The telescope tracks objects at their angular rates. ${ }^{4}$

Observations are done with in a "survey and chase" mode where MODEST is in survey mode and CTI 0.9-m is the chase or follow-up telescope. MODEST observes for 5.3 minutes and the orbit is propagated using a circular assumption to get right ascension, declination, and time. A circular observation is assumed because the initial MODEST observation is not long enough to accurately determine the eccentricity. If MODEST was used for follow-up, it would have to stop survey observations. Thus, a follow-up observation with the CTIO 0.9-m telescope is then made to obtain a longer time arc and get a more accurate orbit. The first follow-up observation typically occurs less than 20 minutes after the MODEST observation. The recovery rates of the follow-up observations are greater than $80 \%$. Most of the observations concentrate on objects fainter than $R=15^{\text {th }}$ magnitude. The data is combined with brighter objects from public catalogs for a complete view of the GEO satellite population. ${ }^{4}$ 


\section{LITERATURE REVIEW}

\subsection{Previous Search Strategy}

This thesis is a continuation of James Biehl's "FORMULATION OF A SEARCH STRATEGY FOR SPACE DEBRIS AT GEO.” The main goal of Biehl's report was to create a search pattern for when an object did not appear in the field of view of the C9 telescope. The search pattern takes into account the best places to observe based on the data as well as the minimizing telescope movement time. The data analyzed in the report used a variation of $\pm 10 \%$ of the classical orbital elements (COE's). This was sufficient to calculate the average maximum variation of inclination, eccentricity, and mean motion that could be allowed and still have the object in the field of view.

The search pattern was dependent upon the COE that is believed to be the largest source of error. Since there were no objects in the data set analyzed in the report that had much variation in the inclination or the right ascension of the ascending node throughout the observations the decision was limited to argument of perigee, mean anomaly, eccentricity and mean motion. It was concluded that mean motion is difficult to plan around because a variation in mean motion could put the object in front, behind, above or below the originally predicted location. Eccentricity somewhat follows the orbital path and this can be used as a search direction; whereas argument of perigee and mean anomaly follow the orbital path exactly and make a good search direction when they are the COE most likely incorrect. The search pattern used for the initial search when an object is missing is a spiral-like motion around the object, which can be seen in Figure 3. The numbers in the figure correspond to the order where the telescope searches for the object. 


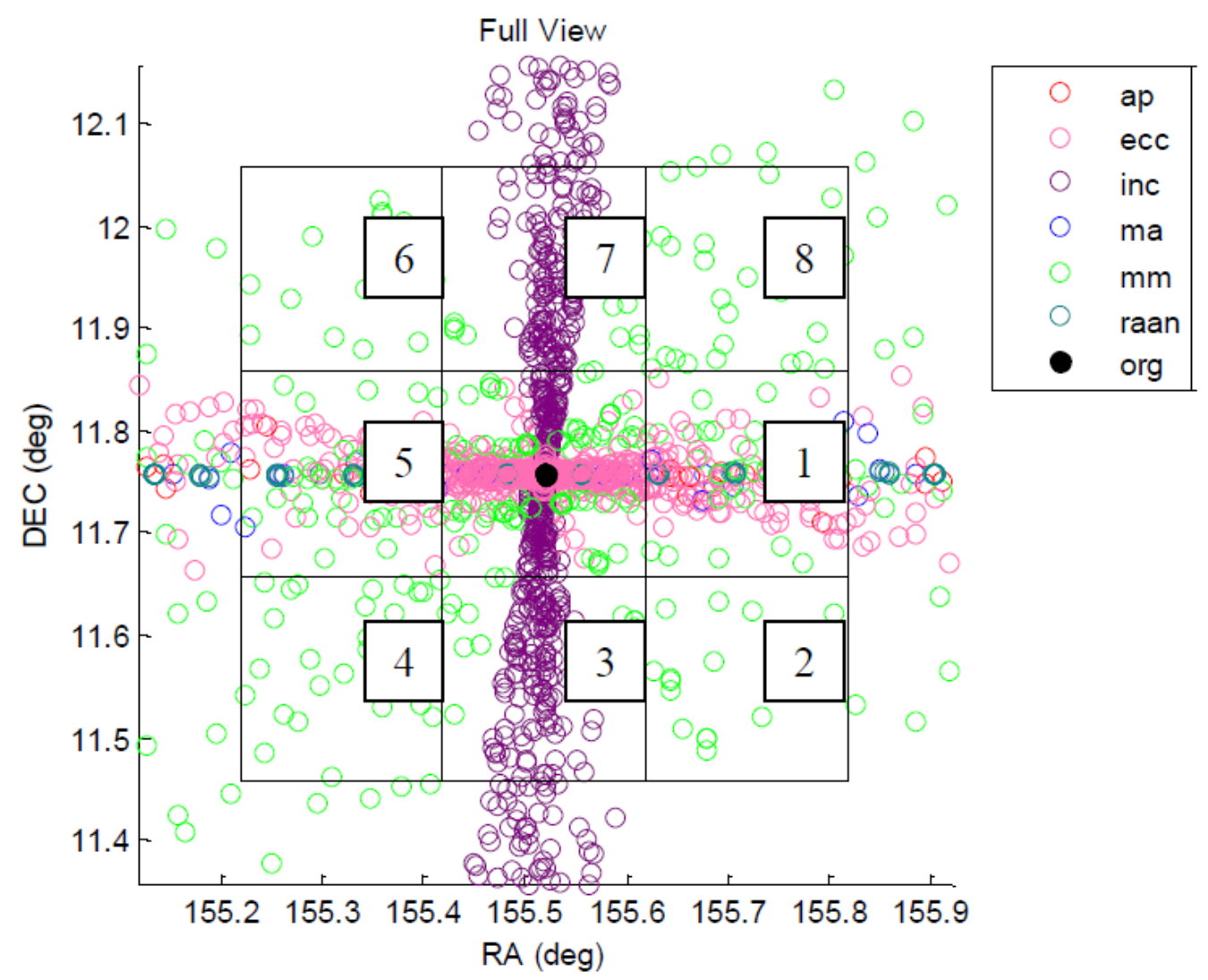

Figure 3. Zoomed in View with search grid for low INC orbits telescope limitations

These search patterns do not take into account the effect of varying multiple COEs at once, which will be the focus of this thesis. The results obtained by Biehl were replicated as a starting point for this thesis and to verify the methods. ${ }^{1}$

\subsection{Asteroid Recovery Review}

There are not many other projects looking at recovering space debris. However, asteroids are often lost and need to be relocated. "The Recovery as an Important Part of NEA Astrometric Follow-up" by J. Ticha, M. Tichy, and M. Kocer, focuses on the recoveries of asteroids at the Klet' Observatory using a 0.57-m telescope equipped with a CCD detector. ${ }^{5}$ Accurate orbit determination requires observations from at least two 
oppositions. If asteroids are not found in the next apparition, different from the discovery apparition, then they can be considered lost. This is particularly embarrassing for near earth asteroids (NEAs). NEA recovery is a very important part of NEA follow-up.

If an object is not found in a field centered on the predicted position, they search along the line of variation of the mean anomaly. In some cases the width of the prediction region is covered by the field of view. Usually such searching area covers \pm 2 fields starting from the field centered on the nominal ephemeris. They search for objects by the ephemeris uncertainty or sometimes the Near Earth Object's Dynamic Site including maps of uncertainty regions (see http://newton.dm.unipi.it/neodys).

Their linear approximation of the prediction region is not bad in many cases, when a short arc asteroid has been lost for a long time, but it sometimes fails, especially in the case of near-Earth asteroids lost not too long ago. Objects that have large ephemeris uncertainty are not searched for but left for a wide-field instrument or survey recovery. Three consecutive images are taken for each searching field. A good set of precise astrometric data is taken to confirm the recovery. They recovered 20 objects from 1999-2001. A graph of the recovered asteroids can be seen in Figure 4. 


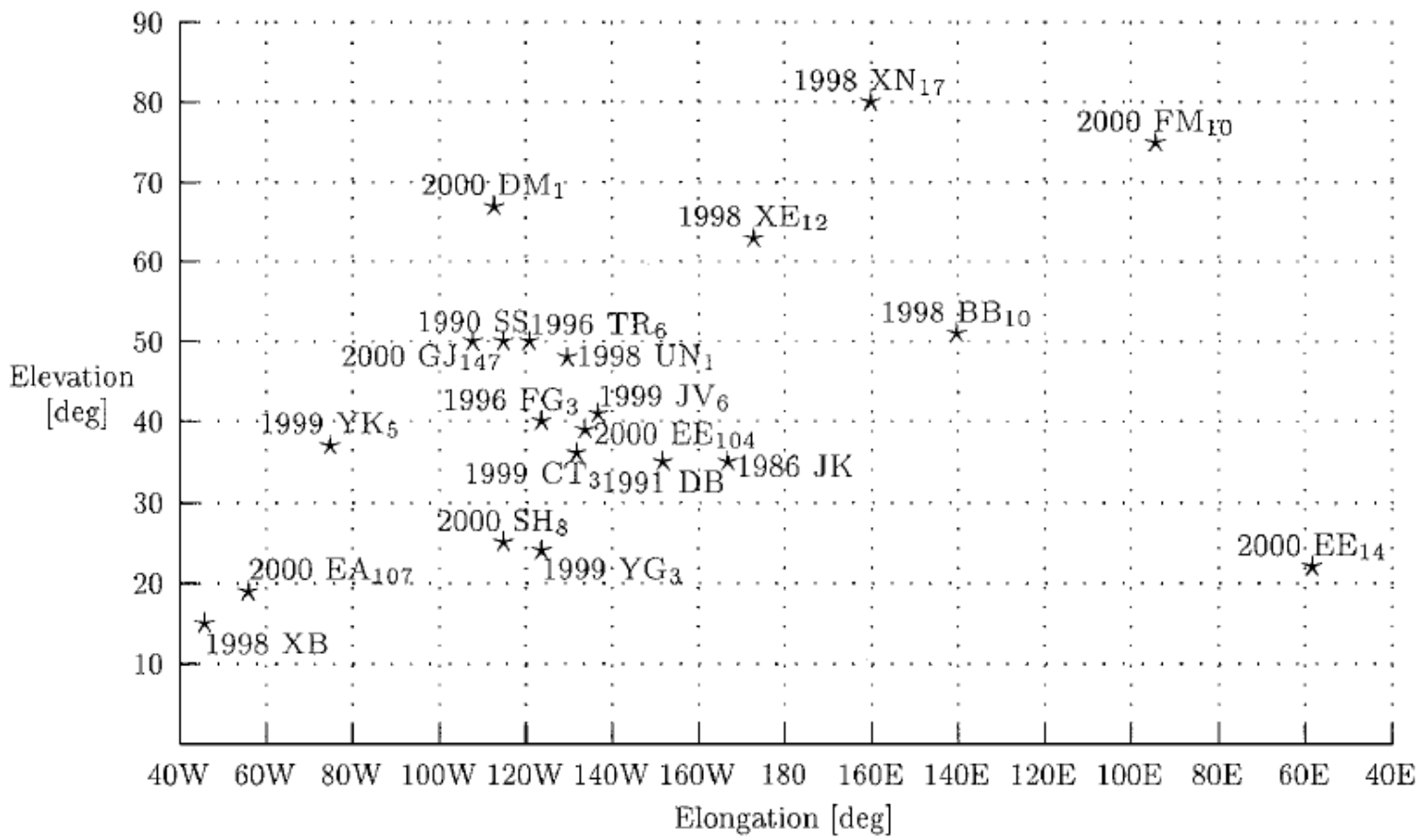

\section{Figure 4. Distribution of Klet' NEA recoveries in the sky.}

In "The Asteroid Identification Problem" by Andrea Milani, the observers need to know the portion of the celestial sphere where an asteroid could be recovered during a given time. ${ }^{6}$ The recovery fails by a large amount when an asteroid has been observed only for short arc or if it has been lost for a long time. The availability of an efficient algorithm to bound the recovery region is essential to finding a lost asteroid and to determine if the recovery effort is worthwhile. Milani developed three algorithms to account for the nonlinearity of their prediction function and allow better predictions on the celestial sphere where lost asteroids could be recovered. The simulated recovery of 9076 PLS, lost in September 1960, can be seen in Figure 5. 


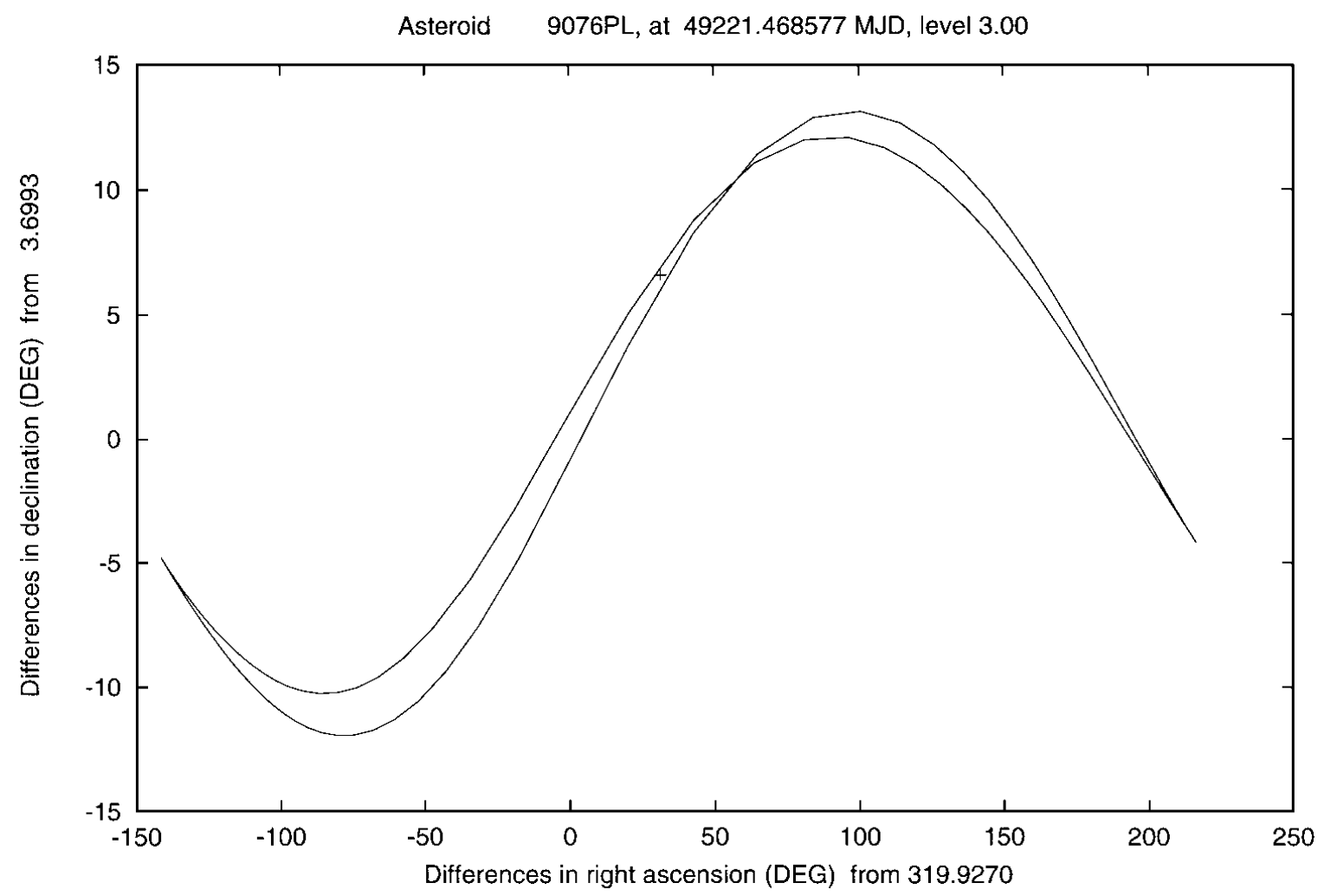

Figure 5. The simulated recovery of the asteroid 9076.

This simulated recovery looks very similar to the right ascension and declination distributions found in this thesis. Their algorithms develop error ellipses where the lost asteroid is most likely to be. The use of the semi-linear confidence boundary accounts for the nonlinearity of the observation function and gives a more reliable boundary curve than the classical confidence ellipse. The covariance matrix for an epoch near the observation times accounts for the nonlinearity of the integral flow. To account for the nonlinearity of the least-squares solution, they have developed an algorithm to compute multiple solutions compatible with the observations. 


\section{ANALYSIS AND DISCUSSION}

\subsection{The data}

For each object MODEST provided data that included RA, DEC, and time values for each observation. There are also predictions from these observations. These predictions are the RA and DEC for future times, COE's, and the two-line element (TLE). Most initial observations are for 5.3 minutes. These observations are used to predict the location of an object at a later time. Initial observations are combined with more observations to create a sufficient orbital arc to predict an accurate orbit.

The data used in this report is 73 different objects from three different years. From 2007, 27 different objects were used, which were observed between 03/13/2007 and 03/26/2007. From 2008, 29 different objects were used, which were observed from 07/31/2008 to 08/08/2008. From 2009, 16 different objects were used, which were observed from 06/18/2009 to 06/26/2009. Most of these objects were not lost, but some were not successfully located on follow-ups. The objects have a designated naming scheme for organization. The first part of the file name is the year and day of year (Ex. 2007214). The second part of the object name is the combined name of the MODEST number and the C9 number. The MODEST number starts at 0001 and increases by 1 . The C9 number starts at 1001 and increases by 1 . The object number is the last two numbers from the MODEST number and the last two numbers from the $\mathrm{C} 9$ number. If an object has an MODEST number of 0001 and a C9 number of 1009, the object number is 0109. The full name of this object would then be 20072140109. JSC numbers are also arbitrarily assigned from 70000 to 71000 for heritage reasons. As the number of 
observations increase the object number changes but the heritage number remains constant.

\subsection{Analysis Summary}

This section will summarize the steps taken to go from an observation file to a probability density graph that shows the most likely location of the object. Observations files with position data for objects were obtained from MODEST. A Fortran script was supplied by NASA to calculate a TLE for each observation. The input to the Fortran script is the observation file, if you are doing a circular or eccentric prediction, what telescope the observations are from, and how long you want the prediction for. The output is the TLE file. This script is run for every observation in the data set.

The function sim_iterate.m returns the original orbital elements (inclination, eccentricity, right ascension of the ascending node, argument of perigee, mean anomaly, and mean motion) and the orbital elements with a random error for each iteration. It also returns the right ascension and declination of the object 20, 40, and 60 minutes after the original time. The input to sim_iterate.m is the file name of the TLE that you want to iterate on.

The function starts by extracting the orbital elements from the input TLE using the elements.m function. The state vector (the position and velocity vector) of original object is then calculated by sv_from_coe.m using the method outlined in Curtis ${ }^{7}$. The function now adds a random normally distributed error to the $\mathrm{x}, \mathrm{y}$, and $\mathrm{z}$ components of the position and velocity vector. This is done by using the MATLAB randn function to generate a random number and multiplying the output by $2 \%$. This creates errors with a normal distribution and a mean of $2 \%$. A unique error is then multiplied to each 
component of both vectors. The vectors now have a normally distributed error on each component with a mean of $2 \%$. The error was originally added to the orbital elements. This was done because the standard deviations for the orbital elements were known for each year from previous measurements. A distribution function was then easily fit to each orbital element. This created clusters of data that were not understood. After communicating with the MODEST team it was concluded that adding error to orbital elements has nonlinear effects, and can cause abnormal results, especially near degenerate values. It was then decided to add the error directly to the state vector. Adding the error directly to the state vector fixed the clustering problem.

The orbital elements of the state vector with random error is then calculated from the state vector using coe_from_sv using the method outline in Curtis ${ }^{7}$. A Fortran script supplied by NASA is then run inside MATLAB. The Fortran script inputs are the start time, how long you want the prediction, and the orbital elements, and it outputs the time, the predicted right ascension, the predicted declination, the right ascension rate of change, the declination right of change, the right ascension acceleration, the declination acceleration, a shadow flag, and a phase angle. Originally the Fortran code required manual inputs by a user. The Fortran code was altered in order to take command line inputs. The Fortran program can then be used in a loop in MATLAB. The code was recompiled using the Visual Studio 2008 Express compiler. The right ascension and declination, orbital elements, and state vectors, for 20, 40, and 60 minutes are calculated and stored. The rest of the data is erased. This process is run in a loop 2000 times, each time calculating new errors for the components of the state vectors. 
For each time frame there is now a data set of 2000 points of right ascension and declination for 20, 40, and 60 minutes after the original object. This represents possible locations of the object if there was a random $2 \%$ error in the state vector. A matrix formatted for insertion into a statistical analysis program called MINITAB was also saved. The original COE's, percent error of each COE, the error in declination, the error in right ascension, and the total RMS error were added in columns to the matrix. This matrix was easily imported into MINITAB for analysis. In order to accurately predict where the object is most likely to be, these 2000 points are used to calculate a probability density function. The probability density function is calculated using a bivariate kernel density estimator by kde2.m. A kernel density estimator is a non-parametric way to estimate the probability density function of a random variable. The kernel density estimator is calculated by

$$
\hat{f}(x)=\frac{1}{n} \sum_{i=1}^{n} K_{h}\left(x-x_{i}\right)=\frac{1}{n h} \sum_{i=1}^{n} K\left(\frac{x-x_{i}}{h}\right)
$$

where $\mathrm{K}$ is the kernel, $\mathrm{h}$ is greater than 0 and a smoothing factor called the bandwidth. Different kernel functions such as uniform, triangular, normal, and biweight can be used. The probability density function is then plotted to determine the most likely location of the object with a $2 \%$ random error.

The probability density function for each object is calculated by having MATLAB cycle through all the objects with mainsim.m and running sim_iterate for the TLE corresponding to that object. A function is also used to calculate the total observing time, the amount of observations, and dates of the observation from the position data. These observations statistics are written to a text file for analysis. A summary of the analysis can be seen in Figure 6 below. 
1. Observations from C9/MODEST for each object

2. NASA Fortran code to get TLE for each object

3. Run mainsim.m

Cycles through all the objects
Runs sim_iterate.m

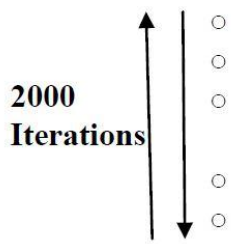

Gets orbital elements from TLE

Run sv_from_coe.m to get state vector

Adds errors with a normal distribution and a mean $2 \%$.

Run coe_from_sv.m to get orbital elements

Run NASA Fortran script to predict RA and DEC

Plots RA versus DEC Distribution

Calculates and plots probability density function and observation information

Figure 6. Summary of the analysis.

\subsection{Results}

All the COE's were varied as described previously. The goal of varying the

COE's is to find the most probable location of debris if it is not in the field of view of the camera. The telescope operator will then know where to move its field of view for the highest chance of recovering the debris. All the COE's were varied randomly with a mean error of $2 \%$. This is in contrast to Biehl's thesis that varied one COE at a time. The output for each object is 2000 pairs of RA and DEC from the simulation. This

distribution is used to find the most likely location of the object. Distributions for objects 70312 and 70302 after 20 minutes can be seen in Figure 7 and Figure 8 below. 


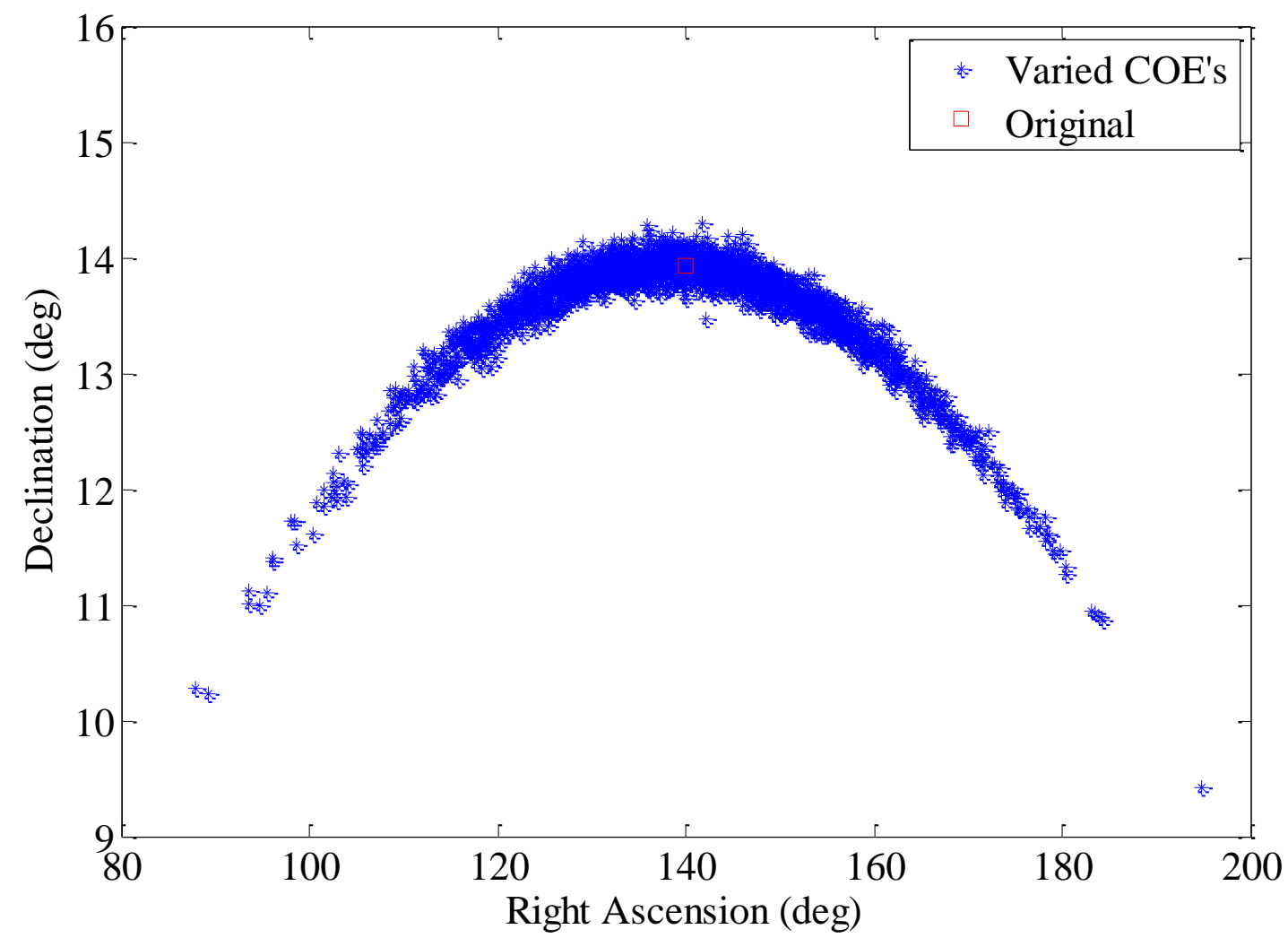

Figure 7. The right ascension versus declination distribution of object 70312 for $\mathbf{2 0}$ minutes after the original time. 


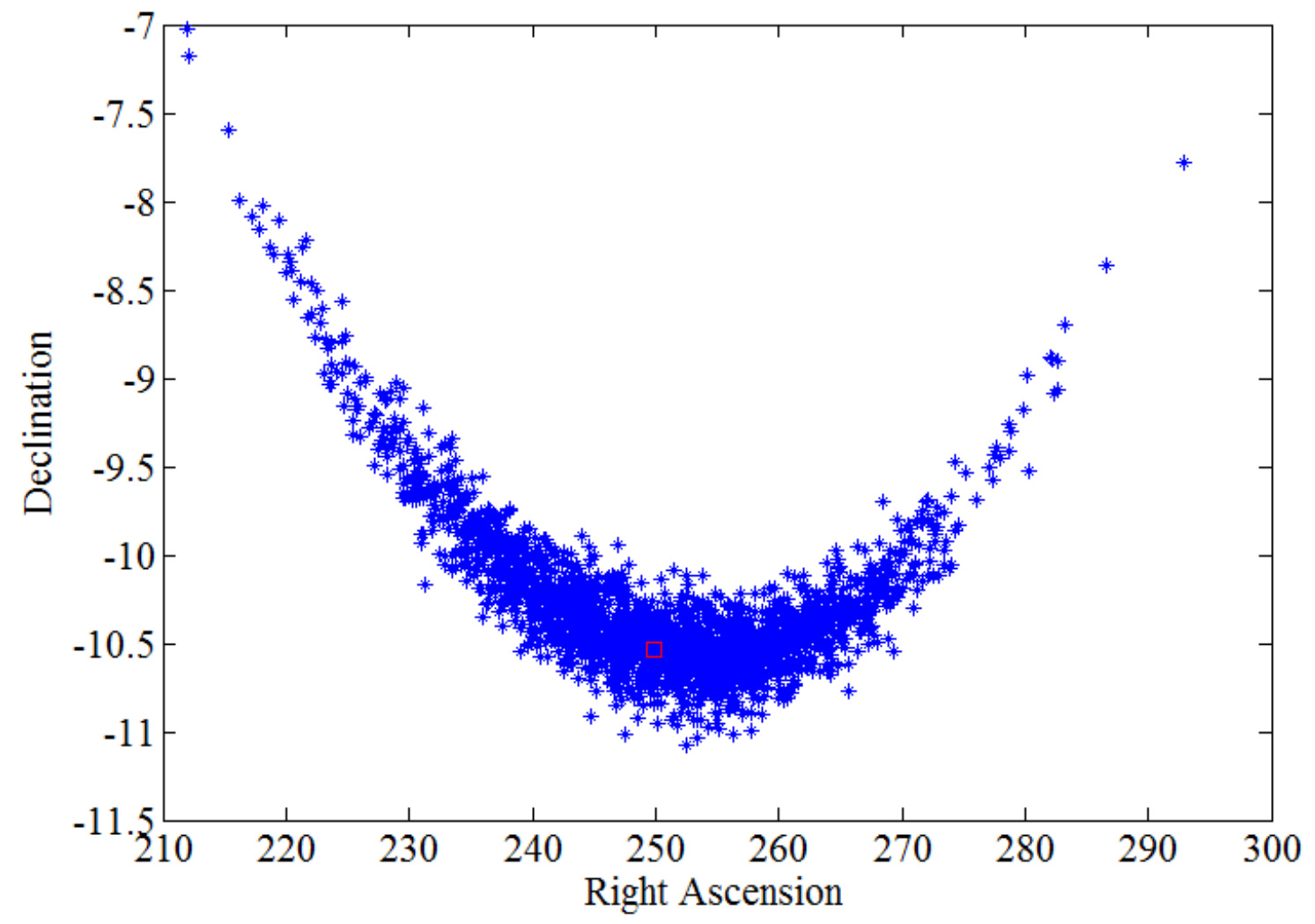

Figure 8. The right ascension versus declination distribution for object 70302 for 20 minutes after the original time.

Each blue star $(*)$ represents the location of the object with a $2 \%$ mean error for one iteration. There are 2,000 blue stars displayed in the graphs above. The red square is the location of the original object with no error added. This distribution was calculated for each object. Object 70312 has a concave up distribution, and object 70302 has a concave up distribution. All objects have similar parabolic distributions. If the distribution covers a wide range of right ascensions, the distribution may have both concave up and concave down components. The right ascension distribution has as big range. The distribution spread can be anywhere from 60 degrees to 360 degrees. The declination typically only varies by 5 degrees or less. It is thus concluded that an object is most likely going to be 
found by moving along the right ascension with a constant declination. Some objects have wider distributions than others given the same $2 \%$ random error, but all follow the same shape. Object 70321 is very sensitive to error and has a large right ascension range of 360 degrees. The distribution has both concave up and concave down sections. The right ascension versus declination distribution of object 70321 for 20 minutes after the original time can be seen in Figure 9.

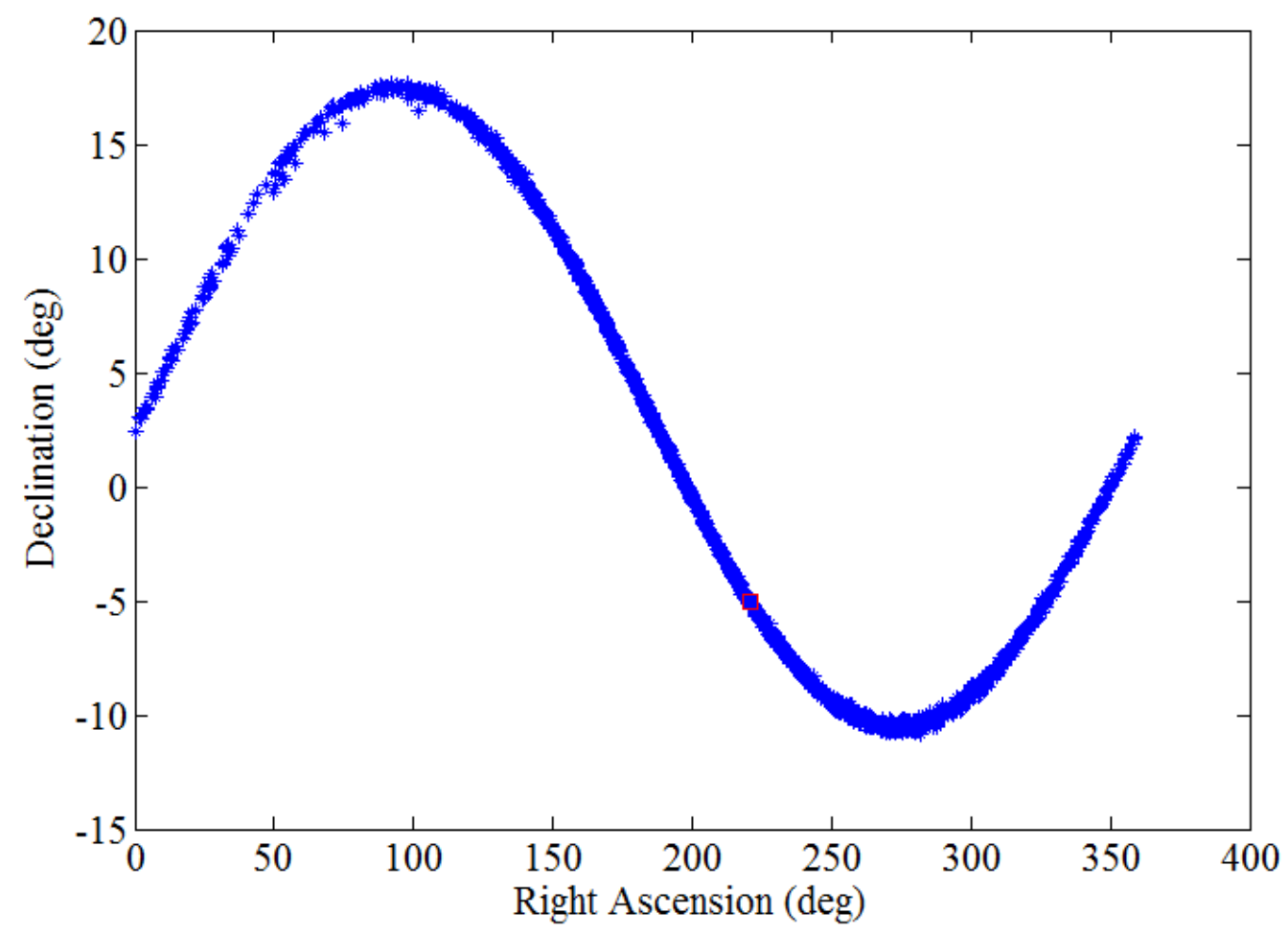

Figure 9. The right ascension versus declination distribution for object 70321.

The original object is found in the middle of the distribution. This makes sense since most iterations will have large errors in only a few orbital elements, placing the object close to the original location. The farther away a simulated point is from the original object, the larger the error on the state vector and orbital elements. 
A bivariate kernel estimator was then used to find the probability density function. The probability density function of the objects 70312 and 70302 after 20 minutes is shown below in Figure 10 and Figure 11.

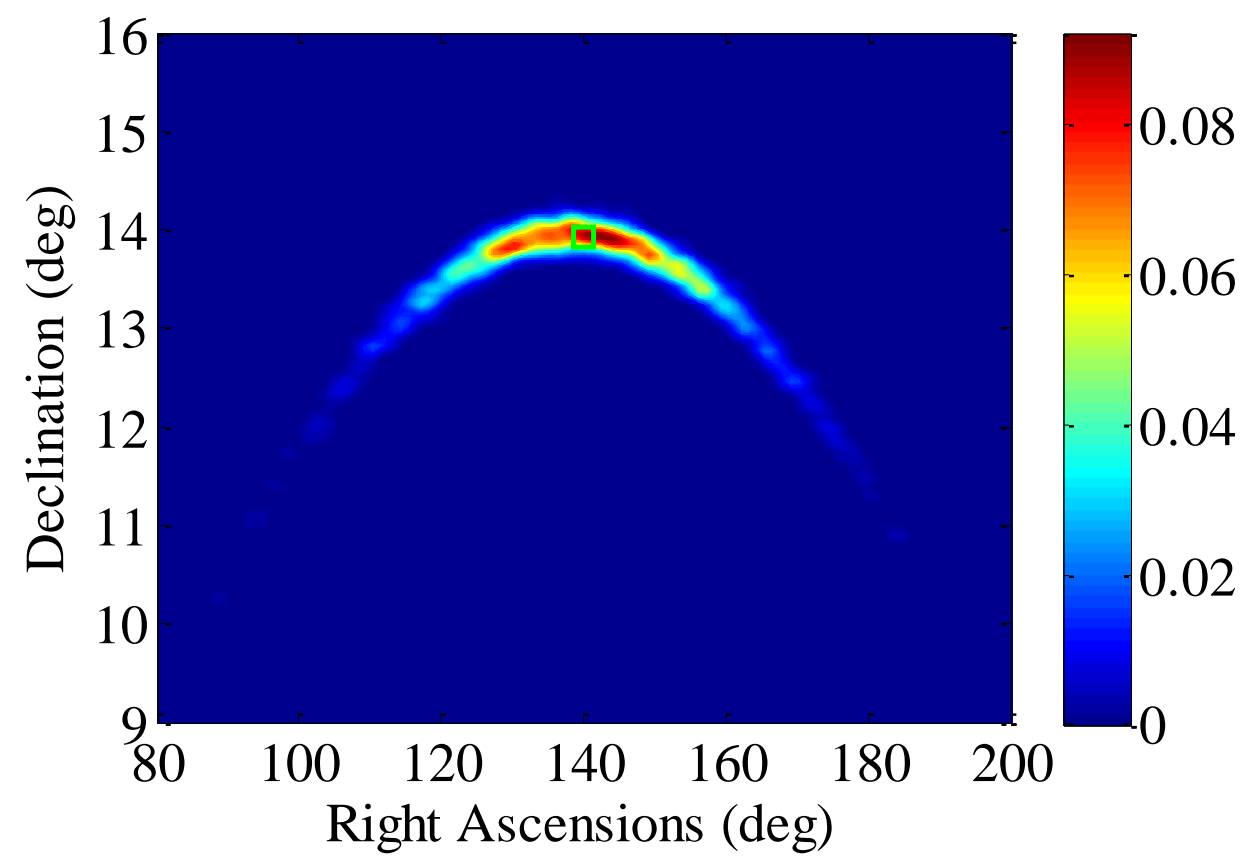

Figure 10. The probability density functions of object 70312 for 20 after the original time. 


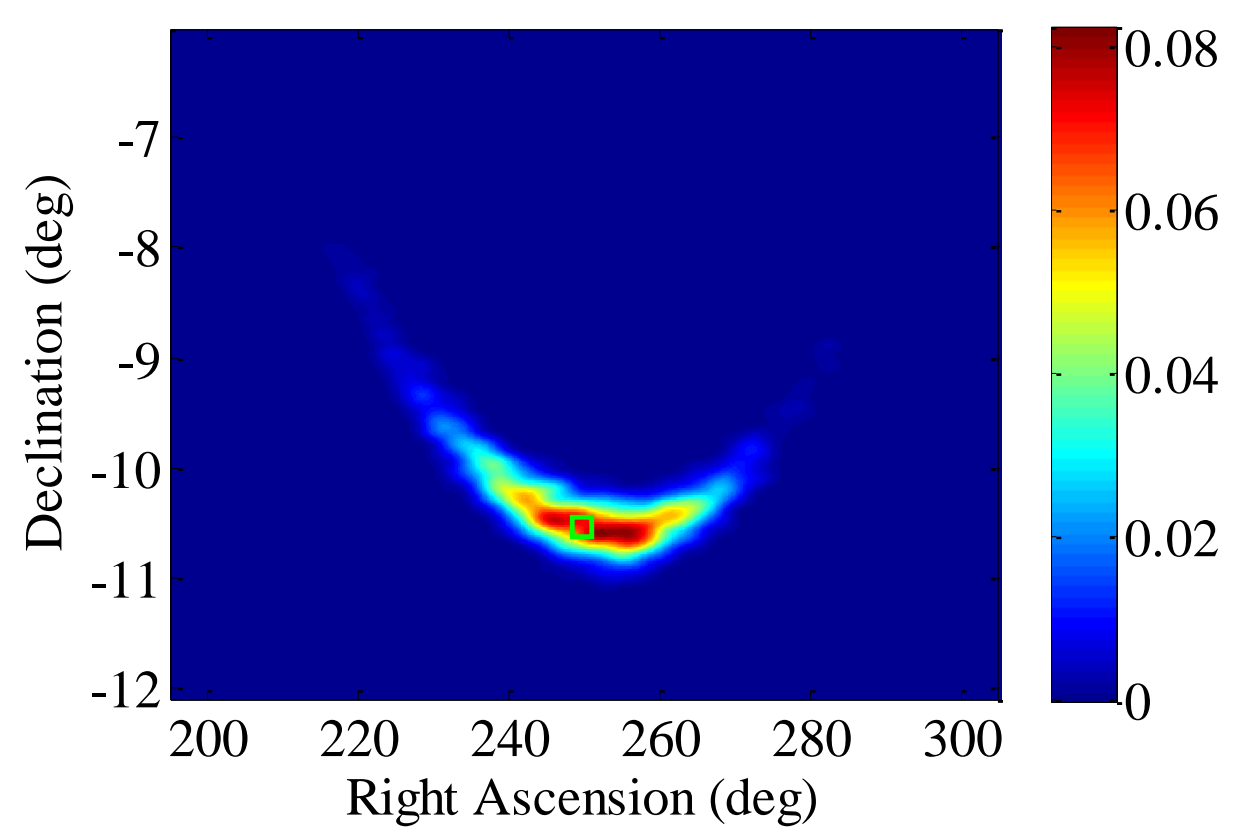

Figure 11. The probability density functions of object 70302 for 20 minutes after the original time.

The green square represents the location of the original object. The colors indicate the probability of the object being in that region. The dark red region is the region of highest probability, and the blue region has the lowest probability. On the right is a legend showing the probability density for each color. The probability density function confirms the conclusions made previously about the distribution. The highest probability is near the original object. The highest probability density is found to the right and left of the original object. Moving the telescope in the right ascension while keeping the declination constant will increase the telescope operator's chance of finding a missing object.

The goal is to find where to move the telescope to find missing objects. Figure 12 and Figure 13 are zoomed in graphs of the above graphs. The zoomed in views show the field of view of the telescope. The telescope has a field of view of $0.22 \times 0.22$ degrees. 
Each square is $0.22^{\circ} \times 0.22^{\circ}$ and represents the field of view of the telescope. The telescope could move its field of view to any of the squares to locate the object.

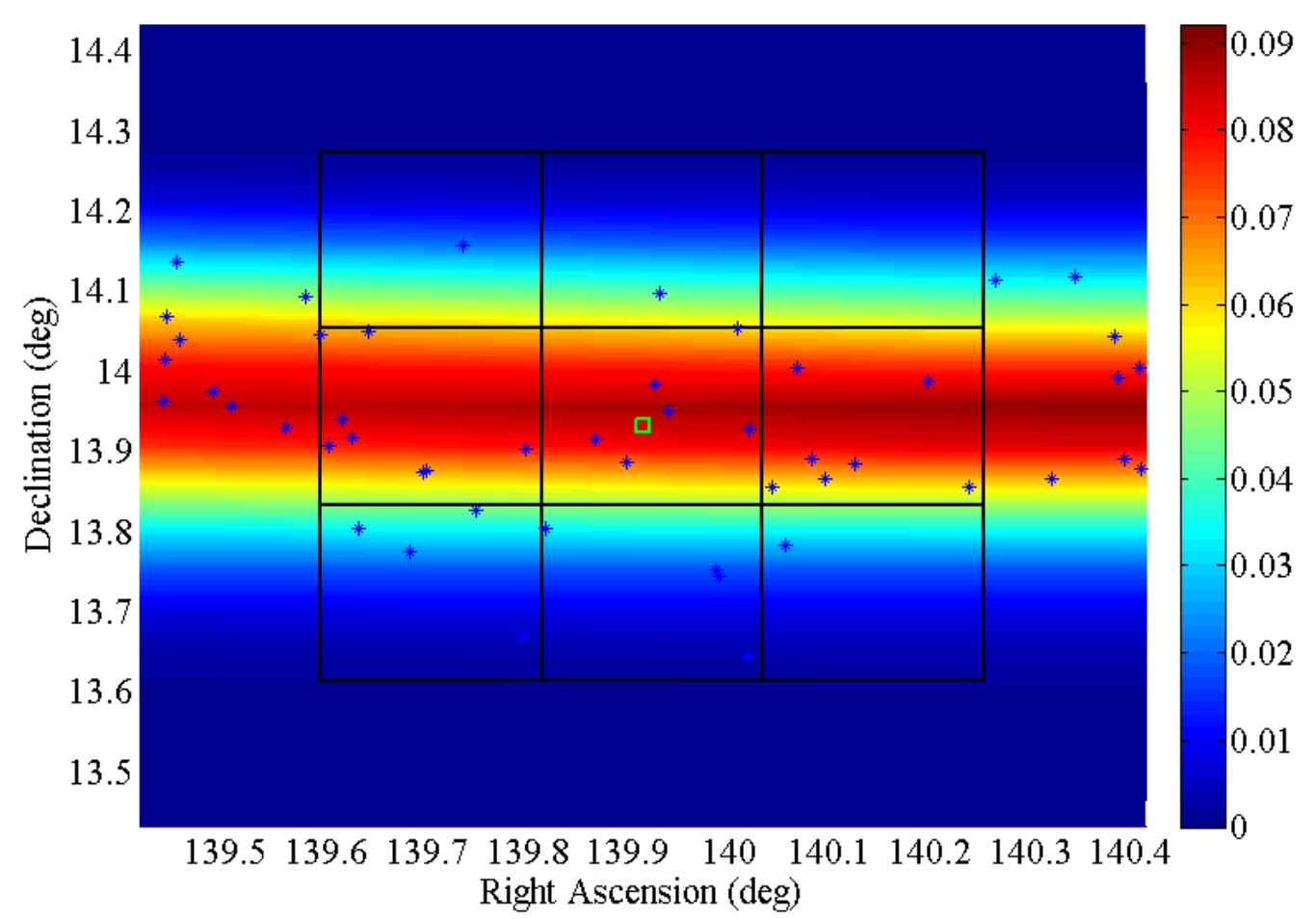

Figure 12. Each square represents the field of view of the telescope with the center square representing the field of view with object 70312 in the center 20 minutes after the last observation. The green square represents the predicted location of object 70312. 


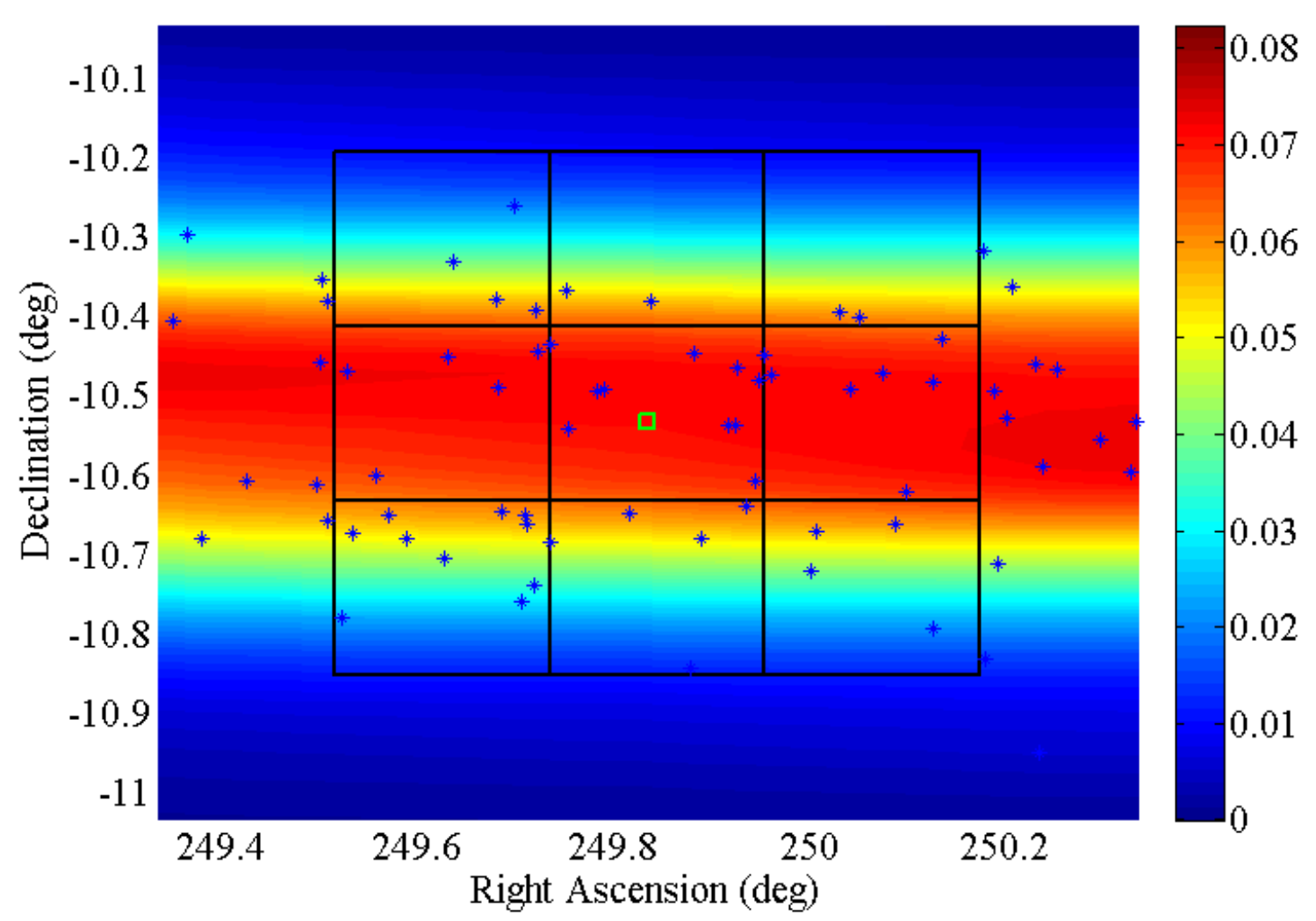

Figure 13. Each square represents the field of view of the telescope with the center square representing the field of view with object 70302 in the center 20 minutes after the last observation. The green square represents the predicted location of object 70302 .

Each blue star $(*)$ represents the location of the object with a $2 \%$ mean error for one iteration. The dark red region is the region of highest probability, and the blue region has the lowest probability. On the right is a legend showing the probability density for each color. As one can see from the graphs above, the most probable locations of the object are directly forward or backwards of the original object in the RA direction (directly to the right or left). This was seen in the previous distribution graphs. These zoomed in graphs are the most useful because it shows the probability density in the field of view of the telescope. This gives the telescope operator a clear indication of where the object is most likely to be. 
The above zoomed in graphs are for two objects. These clearly indicate that the best way to move the telescope is along the right ascension directly to the right or left of the original object. In order to determine the best place for the telescope to look for an arbitrary object, these probability density graphs were created for every object. The goal is to determine which section has the highest probability for every object in the data set. The telescope operator will then know which section the object is most likely to be in for an arbitrary object. The total probability of each square was then calculated, in order to determine which area has the highest probability the most frequently. Each area was designated a letter according to Figure 14.

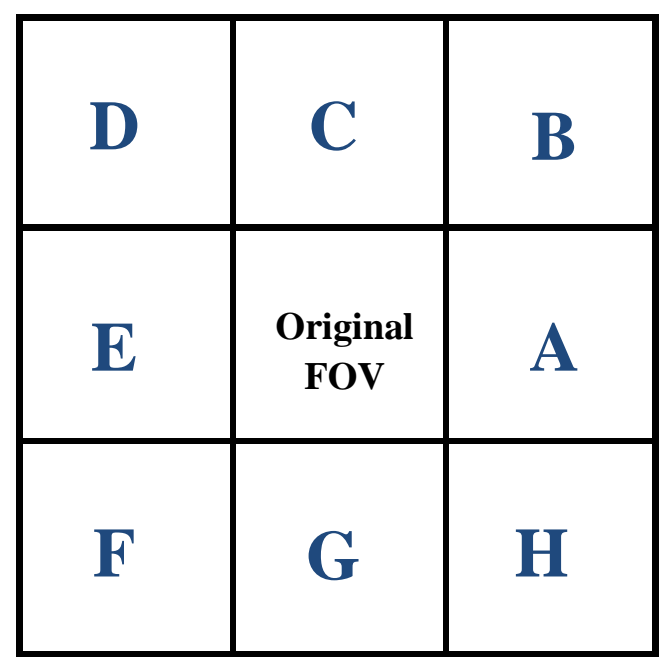

Figure 14. Designations of possible areas for the telescope to move to.

A total of 73 different objects were analyzed. The highest probable area for an object occurred in area A 33 times, in area B two times, in area C zero times, in area D seven times, in $\mathrm{E}$ five 17 times, in area $\mathrm{F}$ zero times, in area $\mathrm{G}$ zero times, and in area $\mathrm{H}$ 11 times. In order to use this data to determine the best place for the telescope to move, this data is represented visually below in Figure 15. 


\begin{tabular}{|c|c|c|}
\hline $7 / 73$ & $0 / 73$ & $2 / 73$ \\
$9.6 \%$ & $0 \%$ & $2.7 \%$ \\
\hline & & \\
$17 / 73$ & Original & $33 / 73$ \\
$23.3 \%$ & FOV & $45.2 \%$ \\
\hline & & \\
$3 / 73$ & $0 / 73$ & $11 / 73$ \\
$4.1 \%$ & $0 \%$ & $15.1 \%$ \\
\hline
\end{tabular}

Figure 15. This figure shows the number of times and percent that each region had the highest probability of finding an object for all 73 objects.

As one can see, region $\mathrm{A}$ and region $\mathrm{E}$ have the highest percentages with $45.2 \%$ and $23.3 \%$ respectively. This figure can be used to determine the optimal search strategy for the telescope. One would want search in the region with the highest probability and move down in descending order. This would make to optimal search order $\mathbf{A} \rightarrow \mathbf{E} \rightarrow \mathbf{H} \rightarrow \mathbf{D}$ $\rightarrow \mathbf{F} \rightarrow \mathbf{B} \rightarrow \mathbf{C} / \mathbf{G}$. Since both $\mathrm{C} / \mathrm{G}$ have the same $0 \%$, their order does not matter. This search pattern can be seen below in Figure 16, where the numbers indicate the order that the telescope moves. The green arrows show the movement pattern the telescope would have to make. 


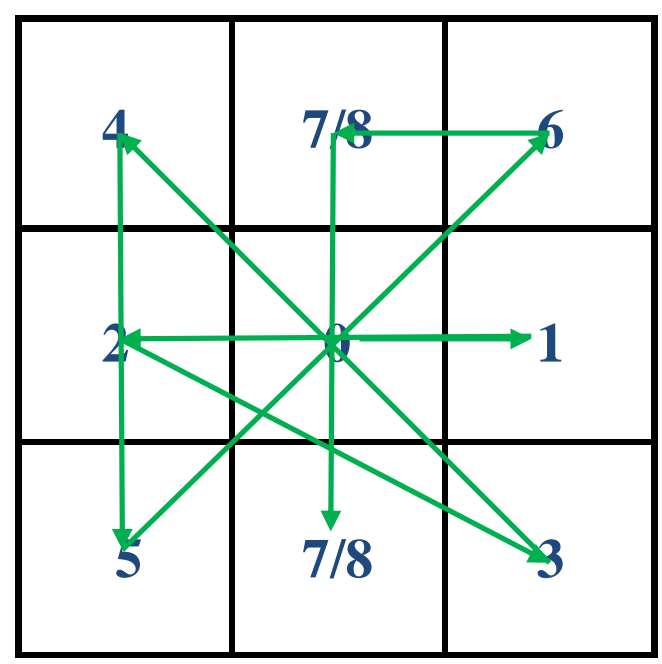

Figure 16. Optimal search strategy for the telescope.

A better search pattern will ignore the regions that have a very small chance of recovery. A better search strategy would be $\mathbf{A} \rightarrow \mathbf{E} \rightarrow \mathbf{H} \rightarrow \mathbf{D}$. This search pattern can be seen below in Figure 17, where the numbers indicate the order that the telescope moves. The green arrows show the movement pattern the telescope would have to make.

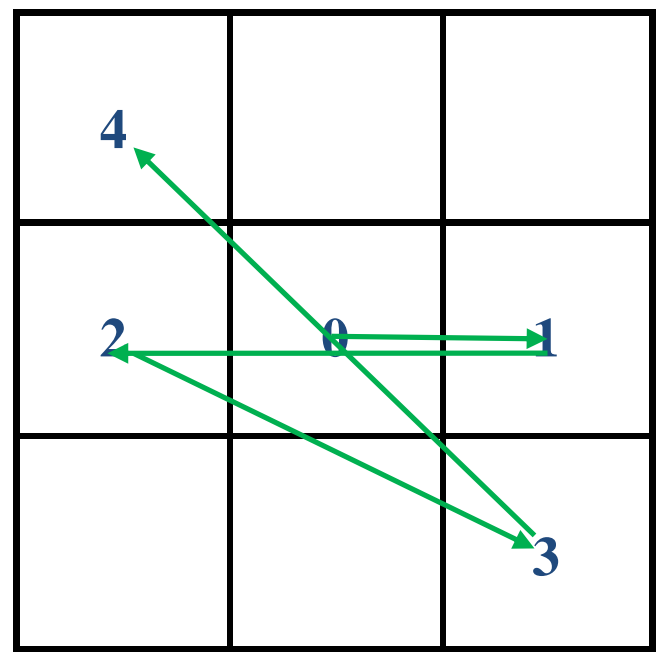

Figure 17. Realistic search strategy given physical constraints of the telescope. 
This is the best search strategy for a typical object without looking at its specific probability density plot. Area's F, B, C, and G are eliminated in this search pattern because the chance of finding them in those regions is very small. Areas F, B, C, and G have small probabilities because the error is added to the velocity vector. The velocity vector is in the direction of the orbit, so adding error should not result in the object appearing in areas F, B, C, and G. The above pattern maximizes the chance of finding an object for a given time. The search pattern is much less complicated than the previous pattern.

\subsection{Graphical User Interface for Onsite Use}

The series of scripts that calculates the probability density function was made into a Graphical User Interface (GUI). This was compiled into an executable .exe using the MATLAB Compiler. The executable can be run on any windows system, and can also be compiled to run on Mac OSX and Linux systems. The MATLAB Deployment Tool was used to make an installation file. Running the setup program will install all the necessary files and libraries to run the exe. The purpose of the program is to be used onsite in Chile at CTIO with a laptop during observations. This program would be run onsite if a followup is unsuccessful. The previous observation data is used to create a TLE. This TLE is then selected with the program, and the program will display the probability density for the given object.

When the .exe is run the program starts and the beginning startup screen can be seen in Figure 18. 


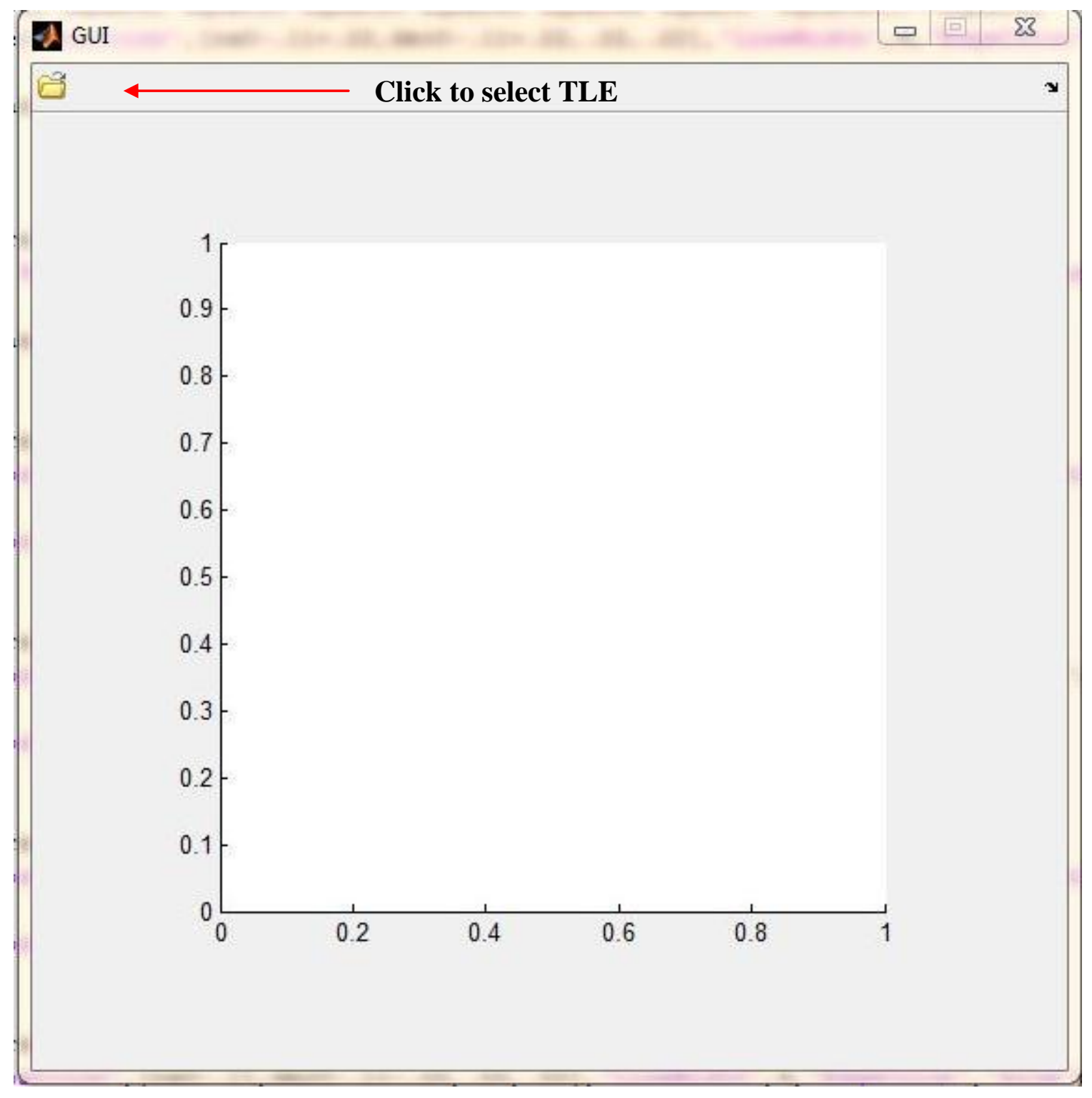

Figure 18. Startup screen of the executable.

The startup screen is simply an empty axis and a menu at the top. Clicking on the file symbol in the upper right will open a browser to select which TLE you want. The program will then calculate the probability density for the selected TLE. The browser that opens to select a TLE is shown in Figure 19. By default this will simulate for 20 minutes after the TLE, but can be changed by the user. 


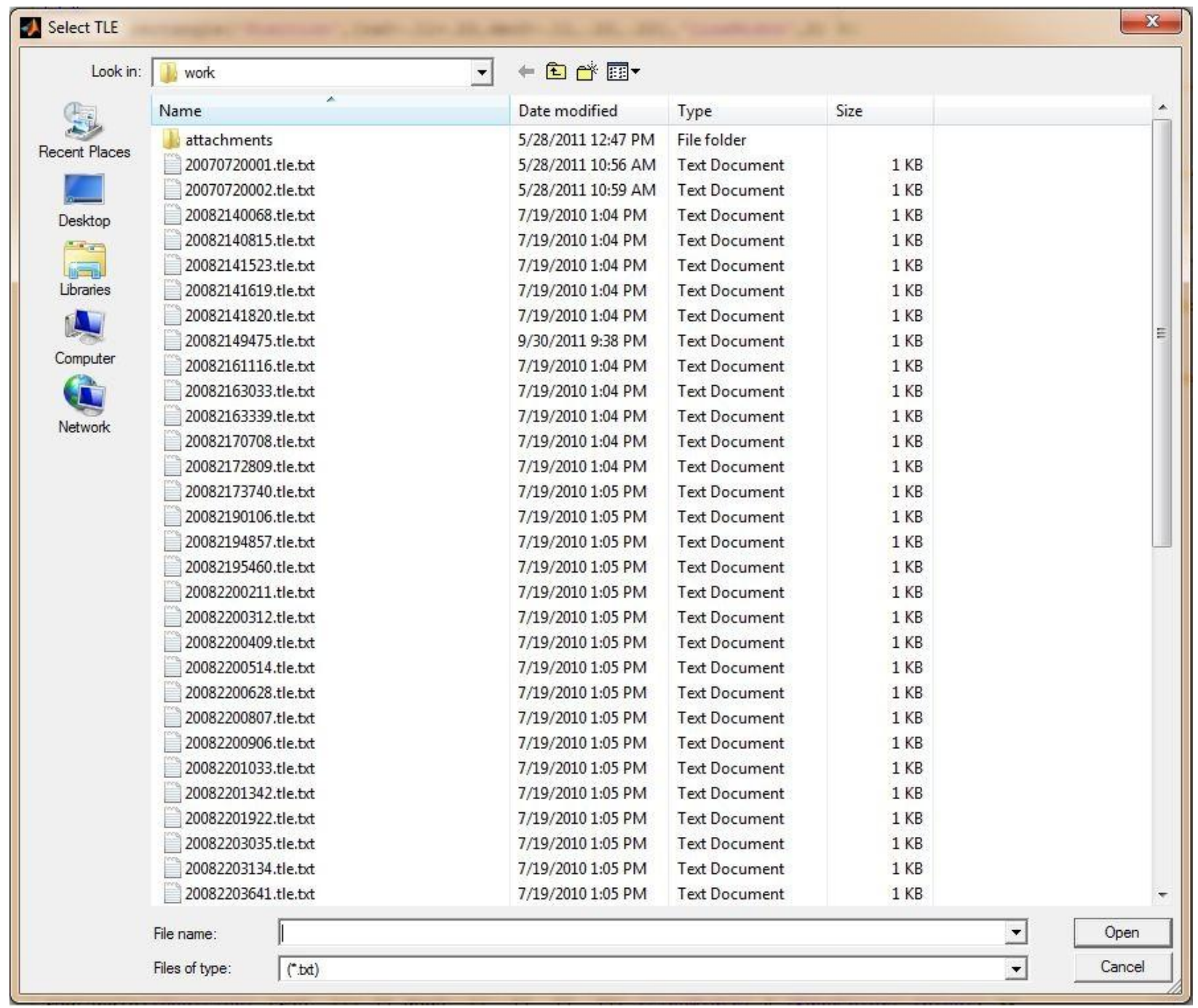

Figure 19. Browser to navigate and select the TLE.

After the file is selected, the program starts running. It iterates 2000 times and should

take about two minutes to run. It will iterate through the sim_iterate.m routine that is

described in the analysis summary (section 3.2). A load bar loads and shows the progress

of the iterations. This can be seen in Figure 20. 


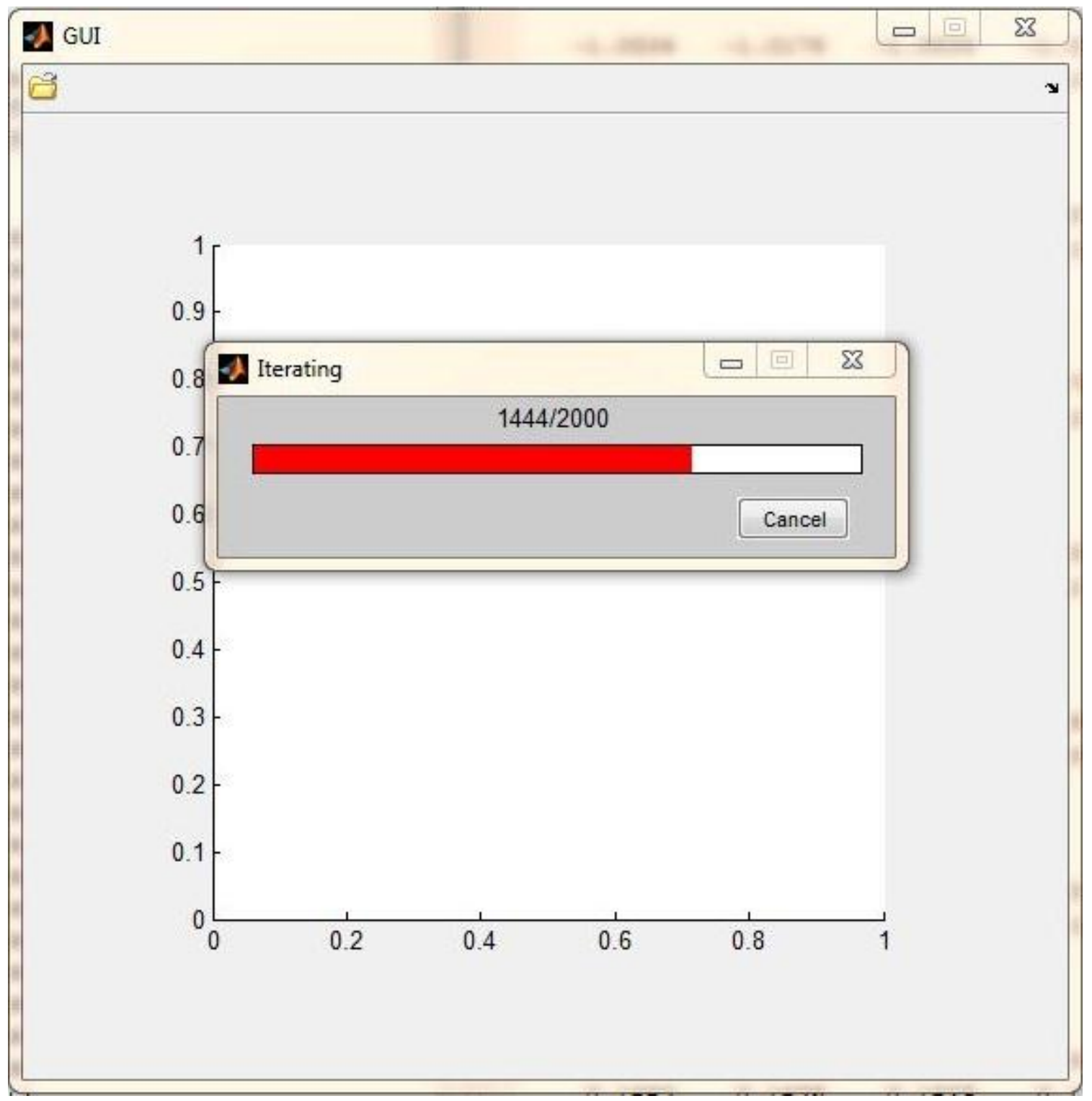

Figure 20. The load bar shows the progress and what iteration the program is on.

Clicking the cancel button on the load bar will cancel the program. Nothing will be shown on the axis if this is done. The $\mathrm{X}$ on the top right of the load bar should not be pressed. Pressing the $\mathrm{X}$ may cause the program to crash, which will require the program to be restarted. This is a problem within MATLAB that the developers are working to resolve. When the program is finished calculating it will display the probability density 
of the chosen object. It will highlight what area has the highest probability with a blue border. This can be seen in Figure 21. The typical iteration time is around two minutes.

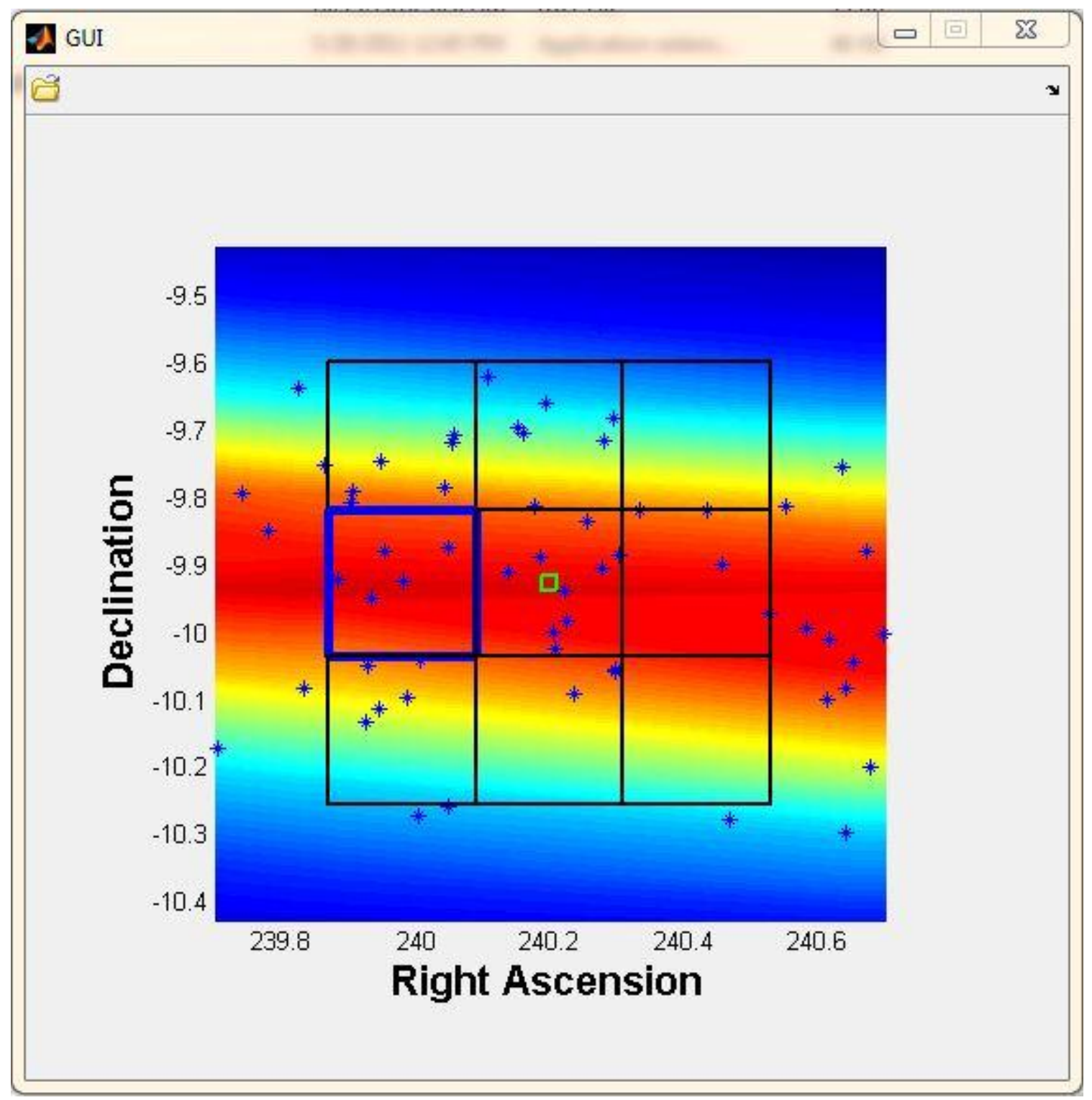

Figure 21. The probability density function displayed in the GUI. The blue square is the region with the highest probability.

This GUI is designed to be used by an observer on site. If a follow-up is unsuccessful the executable GUI can be used to find the most likely location of the object. They can then move the telescope to the most likely location and hopefully find the object. 


\subsection{Search Strategy}

Multiple search strategies have been developed so far. A general search strategy for an arbitrary object was developed in section 3.3. The best place to move the telescope without any analysis is directly to the right or left along the right ascension. The right ascension is much more sensitive to errors than the declination. For most objects, moving to the right, into area $\mathrm{A}$ of Figure 14 is the best choice. If one wants to do a more thorough search one should follow the search pattern shown in Figure 17. This will cover the area with the highest probabilities in the shortest amount of time.

If a laptop is available during observations, more accurate options are available. As outlined in section 3.4, a GUI can be used to calculate the probability density for the given object. The GUI will use the previous observations for a particular object to calculate the probability density. This is a much better choice than following the previous search pattern. The observer can easily determine if the section A from Figure 14 is the best choice, or if the specific object is a rare case where going in a different location will yield better results. Using the GUI lets the observer make the decisions based on what the probability density function looks like. One strategy is to use the GUI to find which initial direction to move the telescope, and then continue in that direction. This can be seen in Figure 22. 


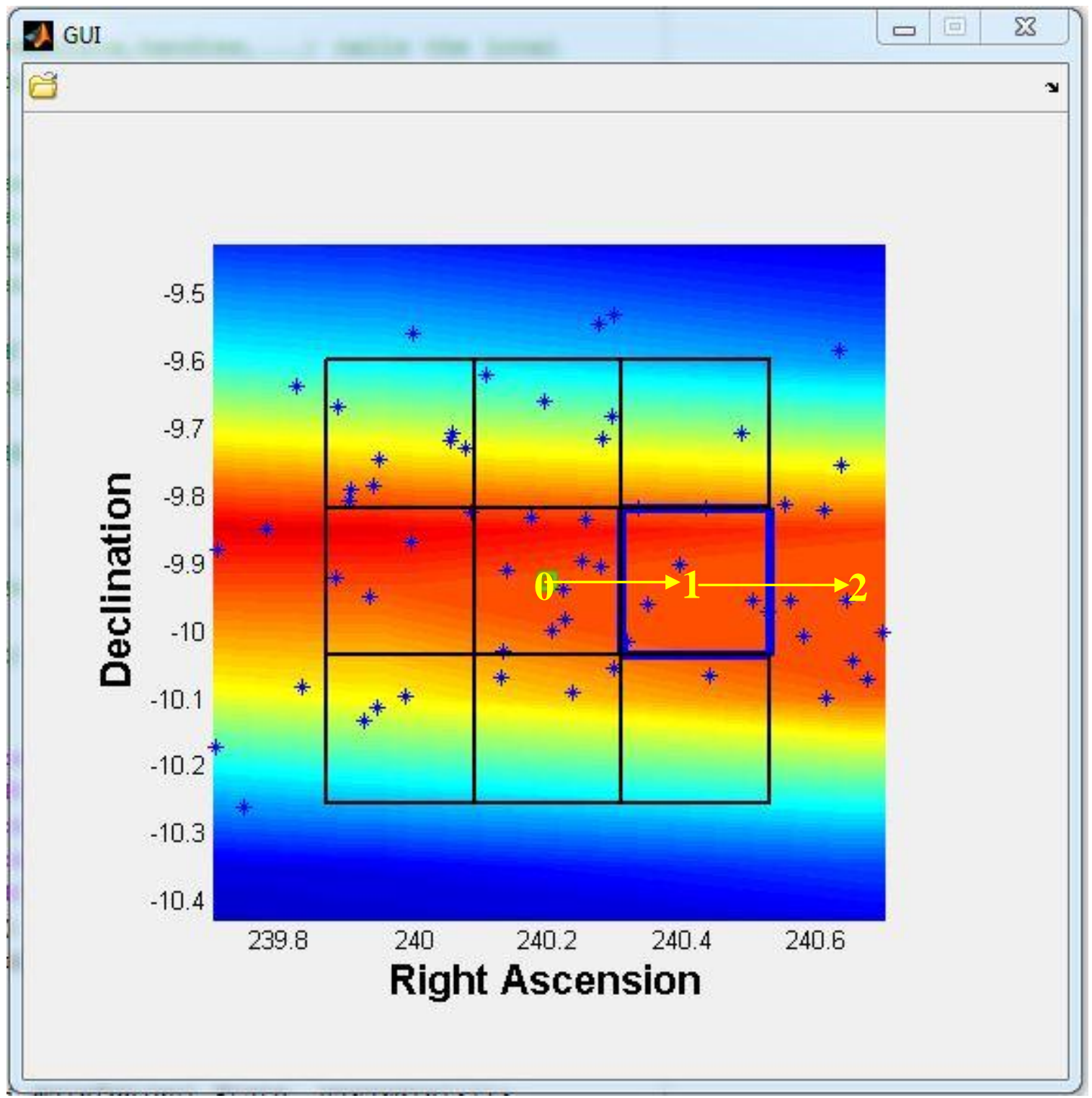

Figure 22. An alternative search strategy for object 70304.

Using the GUI reveals that the area A, directly to the right, has the highest chance of finding the object. The second best choice is to go directly to the left. This requires the telescope to move all the way across areas that have already been viewed. There is a high chance that the area may also be adjacent to area A, which can be seen in Figure 22. The area next to A is as red as area A. For this object it makes sense to move to area to the 
right of area A. Using the GUI will let the telescope operator make better decisions and not use predetermined search patterns.

\subsection{Effect of Simulation Time}

Follow-up observations are usually done 20 minutes after the first observation. After a successful follow-up, the time for the next follow-up varies greatly. The time before another follow-up depends on weather, time, and how many other objects are being tracked that night. It is thus of interest if the time from the original TLE has a great effect on the results. For all 73 objects in the data set, the right ascension and declination distributions for 20, 40, and 60 minutes were saved. This was done to see what effect the simulation time had on the distribution and the areas of highest probability. The right ascension versus declination distribution for object 70312 are shown in Figure 23, Figure 24, and Figure 25. The distributions for each time step are not identical, but they are very close. The distribution has the same shape and range of values. The value of the original prediction for each time step is of course different since the object is moving in its orbit. From the distributions it can be seen that the time does not have a big effect on the distribution shape. 


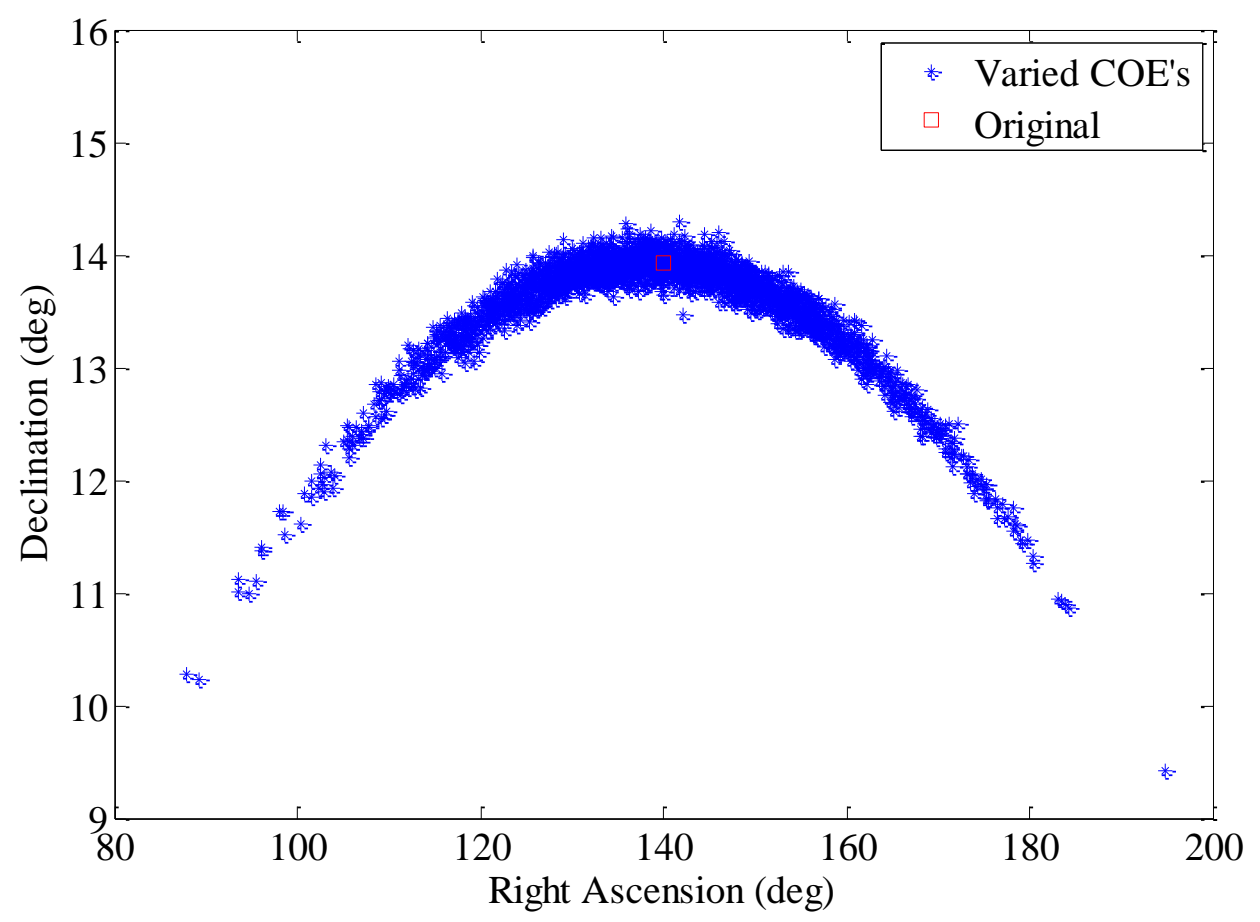

Figure 23. The right ascension versus declination distribution for object 70312 for 20 minutes of simulation time.

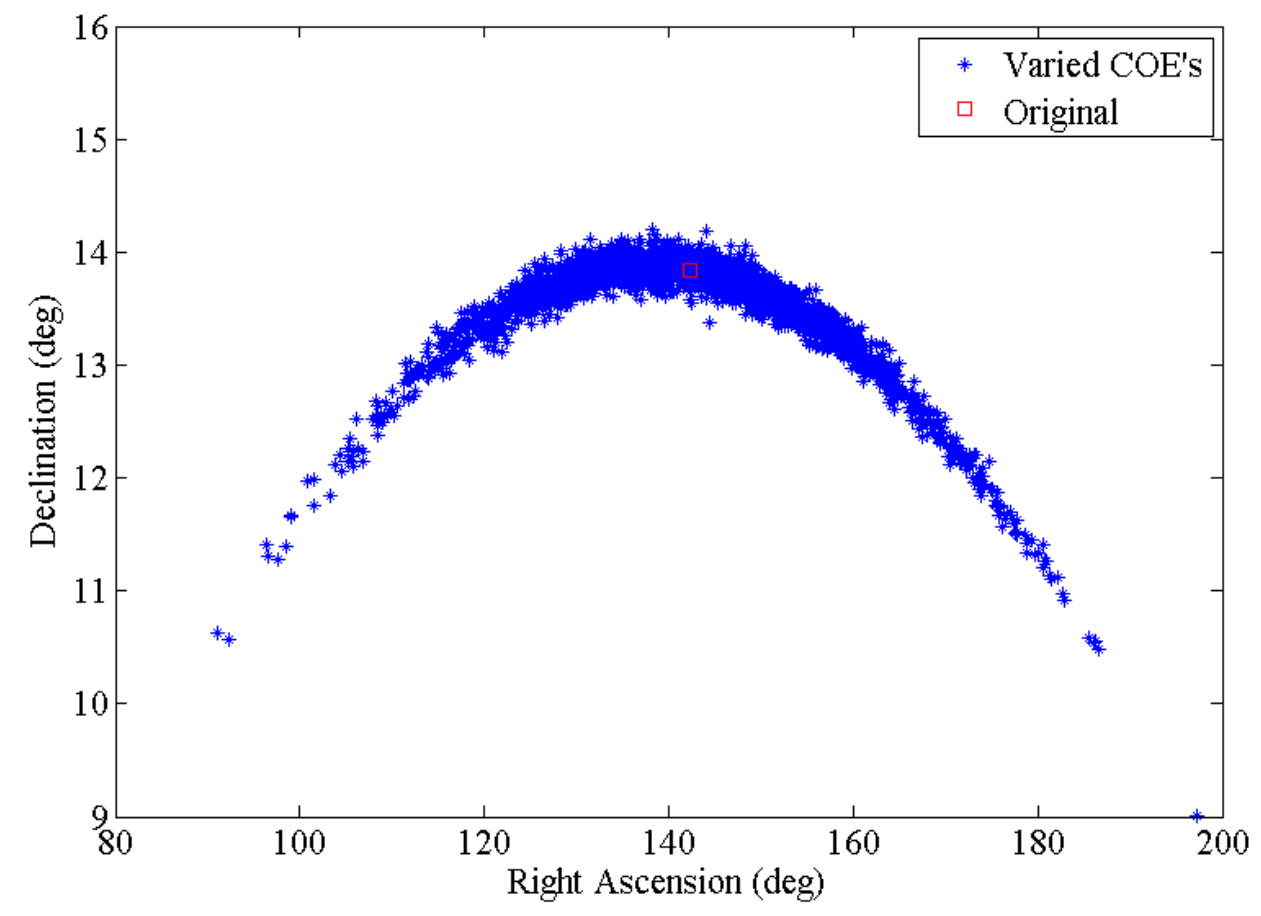

Figure 24. The right ascension versus declination distribution for object $\mathbf{7 0 3 1 2}$ for $\mathbf{4 0}$ minutes of simulation time. 


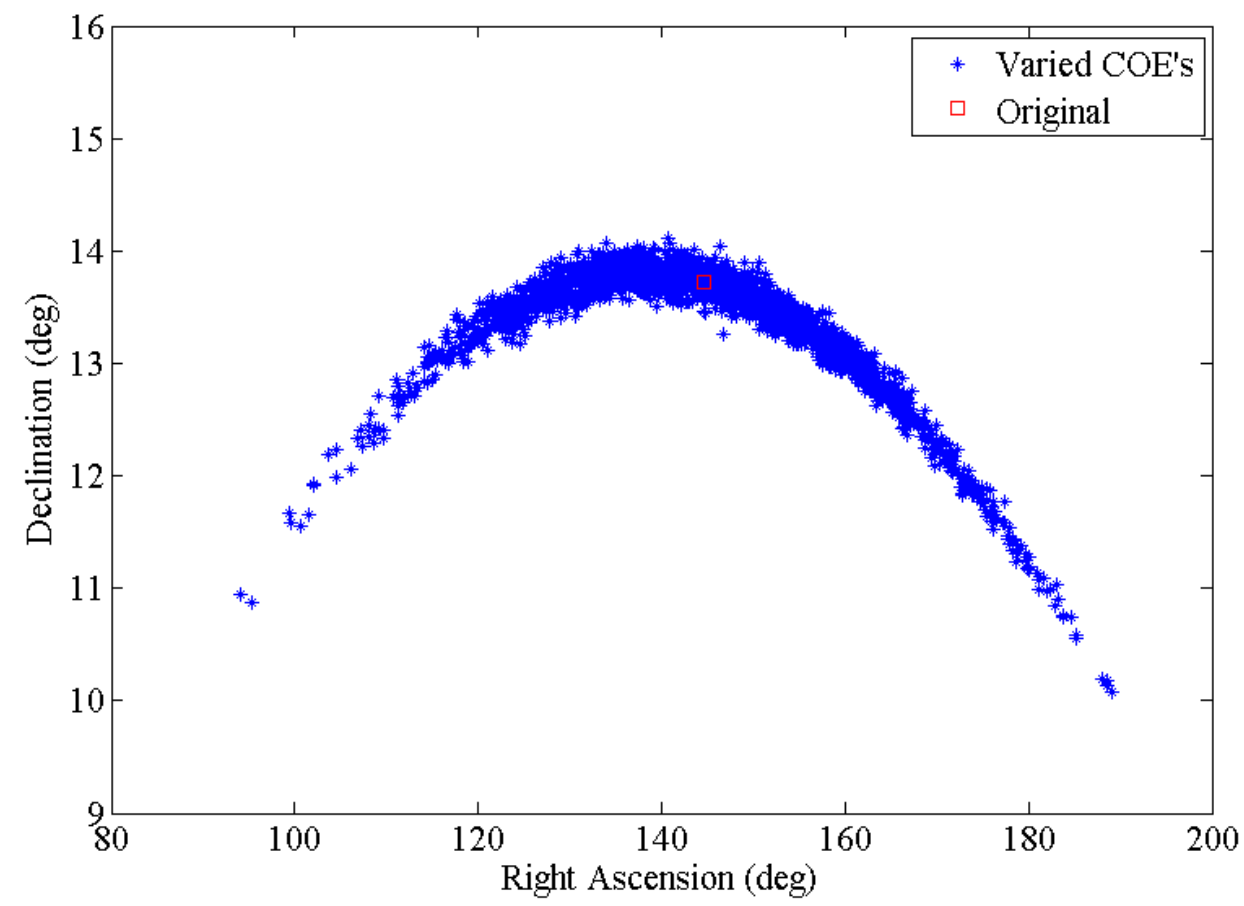

Figure 25. The right ascension versus declination distribution for object 70312 for 60 minutes of simulation time.

The probability density for 20,40, and 60 minutes of simulation time was also saved for every object. The zoomed in field of view of the telescope of the probability densities is shown in Figure 26, Figure 27, and Figure 28. The figures show that the probability density is not very sensitive to time. 


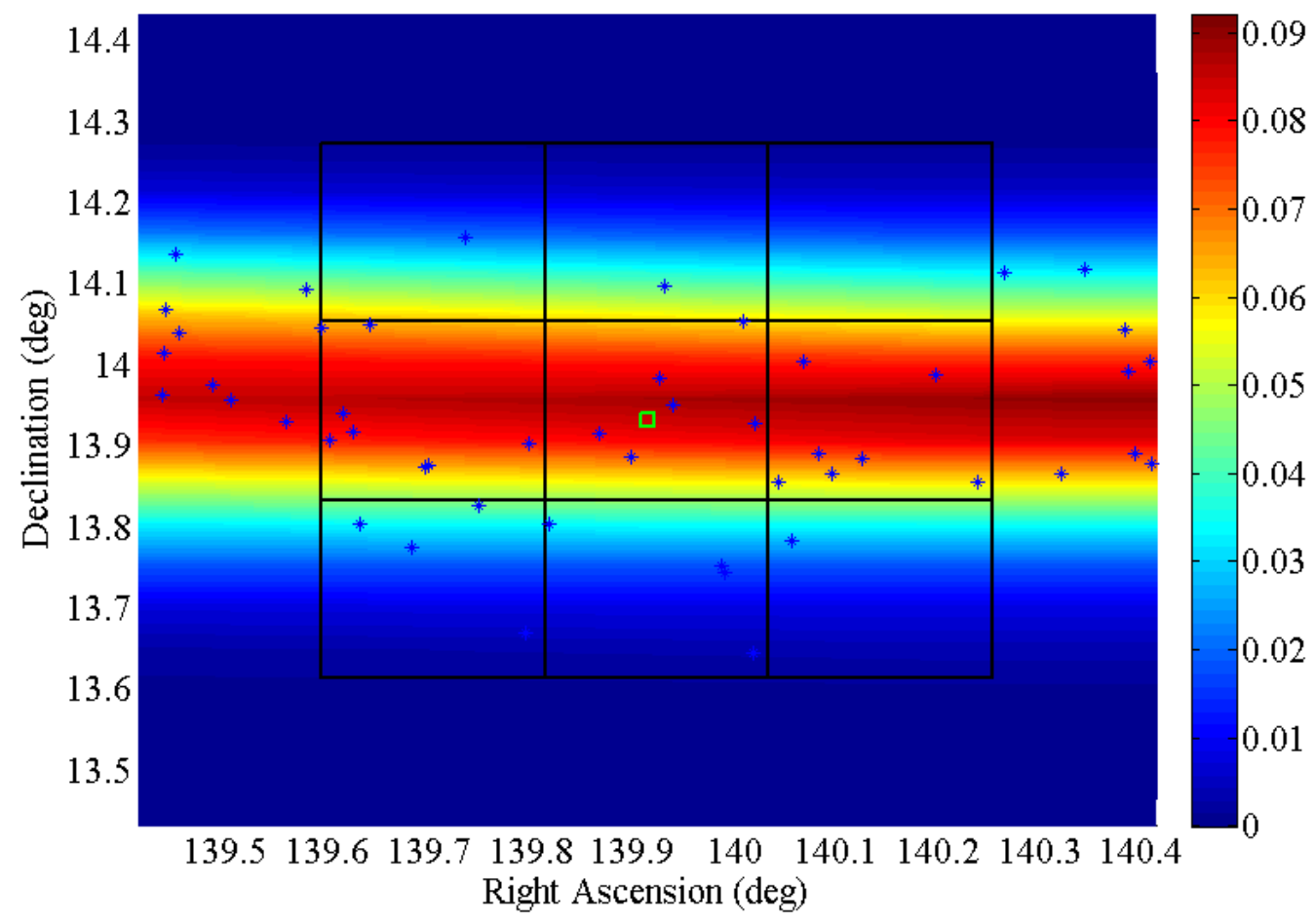

Figure 26. Field of view of the telescope with the center square representing the field of view with object 70312 in the center 20 minutes after the last observation. 


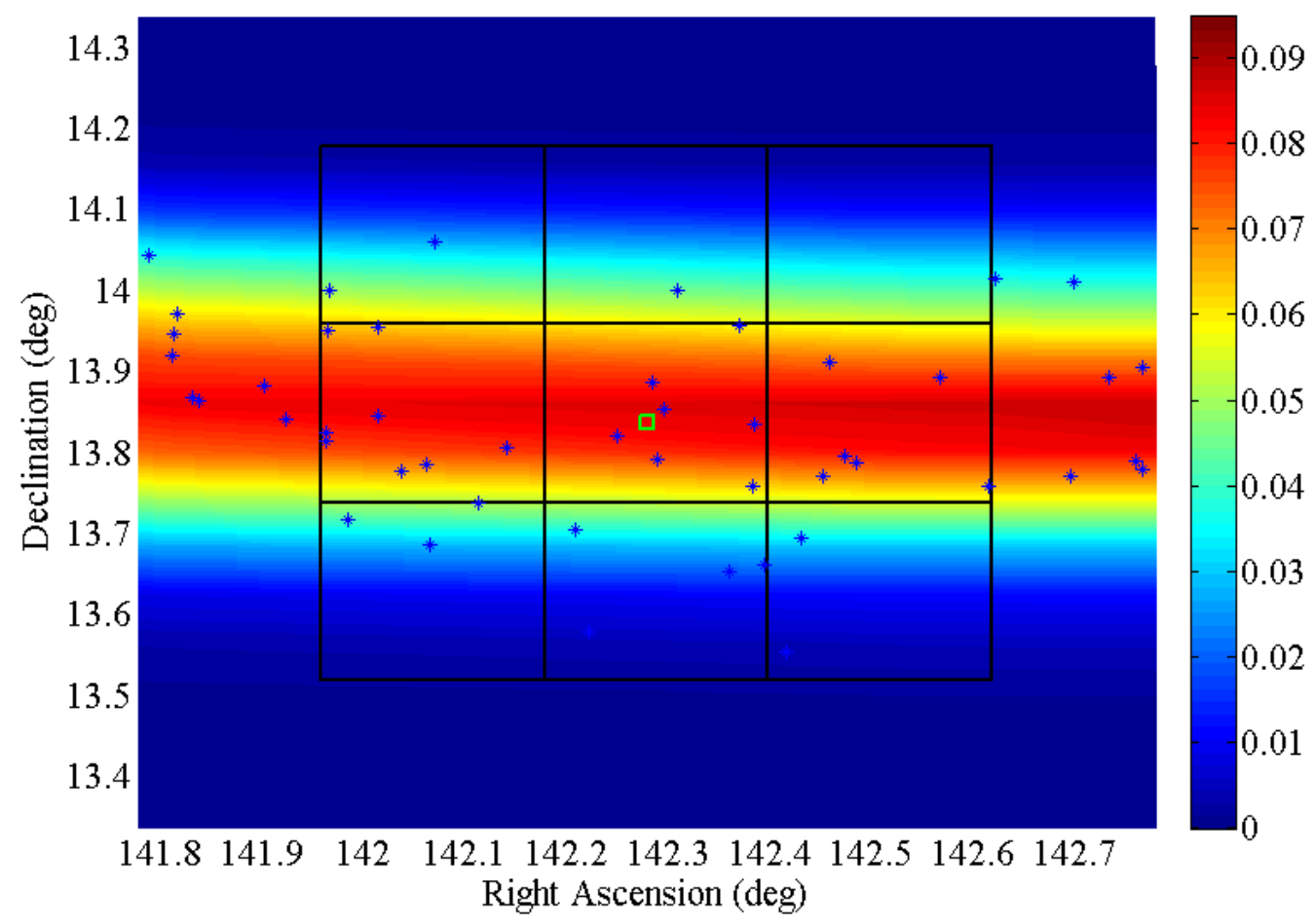

Figure 27. Field of view of the telescope with the center square representing the field of view with object 70312 in the center $\mathbf{4 0}$ minutes after the last observation. 


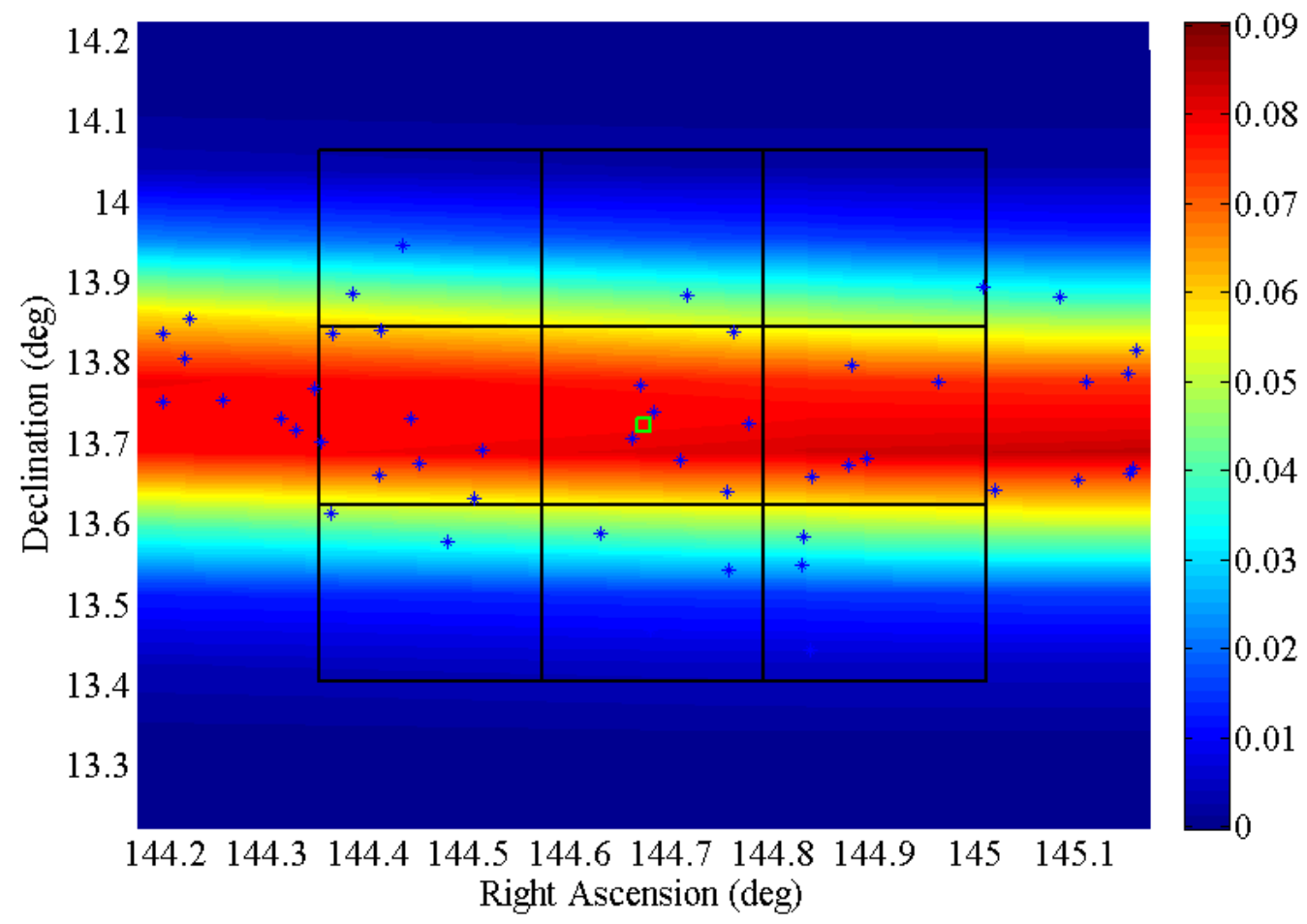

Figure 28. Field of view of the telescope with the center square representing the field of view with object 70312 in the center 60 minutes after the last observation.

The probability densities for each time look very similar. The conclusion that the best maneuver is to move the telescope forward or backwards along the right ascension is still held valid. The distribution and probability densities after 20, 40, and 60 minutes were saved for every object. Looking at all the objects graphs yields the same conclusion. As done in Figure 15 for 20 minutes of simulation time, a script was run to determine the most probable location for 40 minutes and 60 minutes for all objects. The probabilities for each section of the sky for all 73 objects in the data set were identical for 20,40 , and 60 minutes. Time does not have a large impact on which area of the sky to look at for a particular object. 


\subsection{Nonlinear Effects}

The iteration process was originally done by adding the error to the orbital elements, as discussed previously. This caused the RA versus DEC distributions that were odd and could not be explained. This distribution can be seen in Figure 29 below.

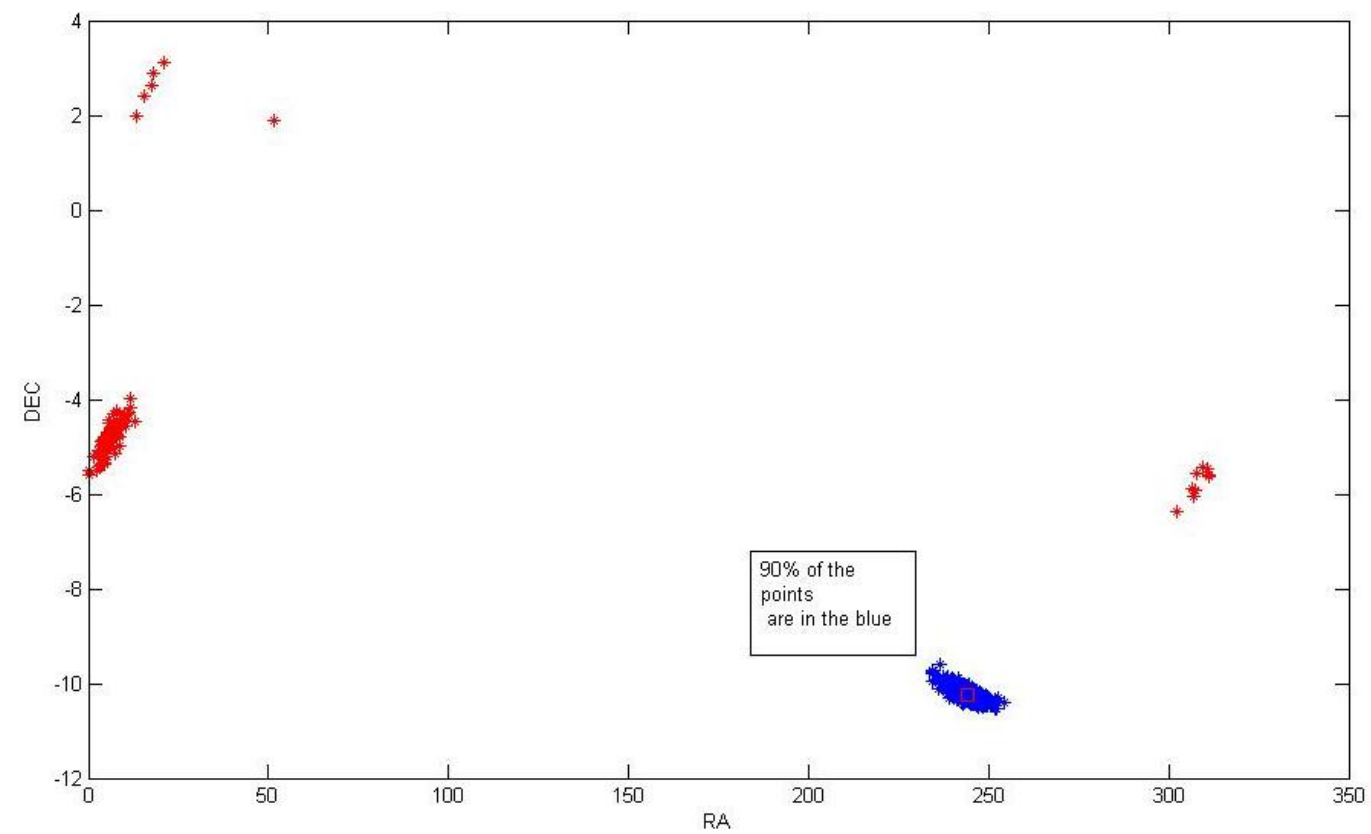

Figure 29. Right ascension versus declination when the error is added to the orbital elements.

When the error was added to the orbital element, there were several distinct groups as can be seen in the graph. In this example over $90 \%$ of the data was near the original object, with $10 \%$ in the other three groups. The specific group distributions varied from object to object, but at least $85 \%$ of the data was in the main group nearest the original object location. There was an effort to determine why these distinct groups occurred. The data points were examined to see if they had any common features such as a high error in a specific orbital element. The groups had no common characteristic. It was decided that 
they were due to nonlinear effects and the error was instead added to the state vector. Adding error to the state vector instead of the orbital elements fixed the group problem.

The MODEST collaboration is interested in knowing which orbital elements cause the most error. If they know which orbital elements cause the biggest error, they can try and get more accurate readings of the most susceptible elements. In order to determine what orbital elements had the most effect on error, a statistical software program called MINITAB was used. The error for calculating the RA error is

$$
R A_{\text {error }}=\frac{R A_{\text {Original Predicted }}-R A_{\text {Iterative }(i)}}{R A_{\text {Original } \text { Predicted }}}
$$

the equation for the error of DEC is

$$
D E C_{\text {error }}=\frac{D E C_{\text {Original Predicted }}-D E C_{\text {Iterative }(i)}}{D E C_{\text {Original Predicted }}},
$$

and the RMS error is calculated by

$$
R M S_{\text {error }}=\sqrt{R A_{\text {error }}^{2}+D E C_{\text {error }}^{2}}
$$

The RMS error is compared to the error in the orbital elements. The inclination error is calculated by

$$
\mathrm{INC}_{\text {error }}=\frac{\mathrm{INC}_{\text {Original Predicted }}-\mathrm{INC}_{\text {Iterative }(\mathrm{i})}}{\mathrm{INC}_{\text {Original Predicted }}}
$$

the eccentricity error is calculated by

$$
\mathrm{ECC}_{\text {error }}=\frac{\mathrm{ECC}_{\text {Original Predicted }}-\mathrm{ECC}_{\text {Iterative(i) }}}{\mathrm{ECC}_{\text {Original Predicted }}}
$$

the right ascension of the ascending node is calculated by

$$
\text { RAAN }_{\text {error }}=\frac{\text { RAAN }_{\text {Original Predicted }}-\text { RAAN }_{\text {Iterative(i) }}}{\text { RAAN }_{\text {Original Predicted }}},
$$

the argument of perigee error is calculated by

$$
\mathrm{AP}_{\text {error }}=\frac{\mathrm{AP}_{\text {Original Predicted }}-\mathrm{AP}_{\text {Iterative }(\mathrm{i})}}{\mathrm{AP}_{\text {Original Predicted }}},
$$


the mean anomaly error is calculated by

$$
\mathrm{MA}_{\text {error }}=\frac{\mathrm{MM}_{\text {Original Predicted }}-\mathrm{MM}_{\text {Iterative }(\mathrm{i})}}{\mathrm{MM}_{\text {Original Predicted }}}
$$

and the mean motion error is calculated by

$$
\mathrm{MM}_{\text {error }}=\frac{\mathrm{MM}_{\text {Original Predicted }}-\mathrm{MM}_{\text {Iterative }(\mathrm{i})}}{\mathrm{MM}_{\text {Original Predicted }}} .
$$

MINITAB was used to fit a model with RMS error as the response variable and inclination, eccentricity, right ascension of ascending node, argument of perigee, mean anomaly, and mean motion as the parameters. Each object had a statistical model fit to it. Each object had different orbital elements that had the most effect on the error. Each object had a statistical model that fit it the best determined by MINITAB, which changed the order of each parameter depending on the object. For object 70304 the statistical analysis is shown in Figure 30. 


\begin{tabular}{|lrrrrrr}
\hline Analysis of Variance & & & & & & \\
Source & DF & Seq SS & Adj SS & Adj MS & F & P \\
Regression & 16 & 3.71182 & 3.71182 & 0.231989 & 4280.2 & 0.0000000 \\
inc_err & 1 & 0.12194 & 0.01768 & 0.017679 & 326.2 & 0.0000000 \\
inc_err*inc_err & 1 & 0.22576 & 0.03361 & 0.033611 & 620.1 & 0.0000000 \\
ecc_err & 1 & 0.72046 & 0.00546 & 0.005460 & 100.7 & 0.0000000 \\
ecc_err*ecc_err & 1 & 0.25105 & 0.00126 & 0.001258 & 23.2 & 0.0000016 \\
ecc_err*ecc_err*ecc_err & 1 & 0.00239 & 0.00460 & 0.004601 & 84.9 & 0.0000000 \\
ran_err & 1 & 0.06141 & 0.00081 & 0.000812 & 15.0 & 0.0001122 \\
ran_err*ran_err & 1 & 0.00783 & 0.00124 & 0.001235 & 22.8 & 0.0000019 \\
ap_err & 1 & 0.35803 & 0.01410 & 0.014096 & 260.1 & 0.0000000 \\
ap_err*ap_err & 1 & 1.05908 & 0.02273 & 0.022732 & 419.4 & 0.0000000 \\
ap_err*ap_err*ap_err & 1 & 0.03099 & 0.01402 & 0.014020 & 258.7 & 0.0000000 \\
ma_err & 1 & 0.03702 & 0.00661 & 0.006612 & 122.0 & 0.0000000 \\
ma_err*ma_err & 1 & 0.00848 & 0.00705 & 0.007045 & 130.0 & 0.0000000 \\
ma_err*ma_err*ma_err & 1 & 0.03350 & 0.00689 & 0.006894 & 127.2 & 0.0000000 \\
mm_err & 1 & 0.16923 & 0.00511 & 0.005110 & 94.3 & 0.0000000 \\
mm_err*mm_err & 1 & 0.62174 & 0.62423 & 0.624226 & 11517.0 & 0.0000000 \\
mm_err*mm_err*mm_err & 1 & 0.00290 & 0.00290 & 0.002902 & 53.6 & 0.0000000 \\
Error & 1983 & 0.10748 & 0.10748 & 0.000054 & & \\
Total & 1999 & 3.81930 & & & & \\
& & & & & &
\end{tabular}

\section{Figure 30. The statistical analysis of object 70304 from MINITAB.}

For this object the eccentricity, argument of perigee, mean anomaly, and mean motion have a statistically significant third order relationship with the RMS error. The eccentricity has a p-value of $\mathrm{p}=0.000000$ and an F-statistic of $\mathrm{F}(1,1983)=84.9$, the argument of perigee has a $p$-value of $\mathrm{p}=0.000000$ and an $\mathrm{F}$-statistic of $\mathrm{F}(1,1983)=258.7$, the mean anomaly has a $p$-value of $p=0.000000$ and an F-statistic of $F(1,1983)=127.2$, and the mean motion has a $\mathrm{p}$-value of $\mathrm{p}=0.000000$ and an F-statistic of $\mathrm{F}(1,1983)=53.6$. The inclination and the right ascension of the ascending node have a statistically significant second order relationship with the RMS error. The inclination has a p-value of $\mathrm{p}=0.000000$ and an F-statistic of $\mathrm{F}(1,1983)=326.2$, and right ascension of the ascending node has a $\mathrm{p}$-value of $\mathrm{p}=0.0000019$ and an F-statistic of $\mathrm{F}(1,1983)=22.8$. Looking at the adjusted SS one can see what orbital elements had the most effect on the RMS error. One can see that the mean motion and argument of perigee have the most 
effect. Which orbital elements affected the RMS error the most did not have a pattern.

Each object had different results. This provides evidence that the orbital elements have a nonlinear and complicated relationship to the right ascension and declination as suspected from Figure 29. 


\section{CONCLUSION}

In this paper the COE's were varied to find the most probable location of space debris when the debris is not found in the field of view of the CTIO C9 telescope. All the COE's were varied randomly with a mean random error of $2 \%$. The output for each object is 2000 pairs of RA and DEC from the simulation. Several search strategies were developed in this paper. Search strategies were developed by examining a data set of 73 objects that were observed in Chile from 2007-2009.

A general search strategy was developed based on the statistics of the data set. In general following the search pattern of Figure 17 will give the highest probability of finding an object in the shortest amount of time. For a specific object, a program was developed for calculating the probability density based on the previous observations. The purpose of the program is to be used on site at CTIO with a laptop during observations. This program would be run if a follow-up is unsuccessful. The program would tell the observer which area of the sky the object has the highest probability to be in. The observer can then develop a search strategy based upon the probability density graph.

The COE's were all varied at the same time by adding a $2 \%$ error to the position and velocity vectors. This is done by using the MATLAB randn function to generate a random number and multiplying the output by $2 \%$. This creates errors with a normal distribution and a mean of $2 \%$. A unique error is then multiplied to each component of both vectors. The vectors now have a normally distributed error on the position and state vector with a mean of $2 \%$.

A correlation between error in the orbital elements and error in right ascension and declination RMS error were investigated. It was found that the orbital elements affect 
the RMS error nonlinearly. However, the exact relation between orbital element and error depended on the object and no general pattern was found. It was found that how long after the original object the follow-up was attempted did not have an impact on the probability density function or the search strategy. 


\section{References}

[1] Biehl, James P. "Formulation of a Search Strategy for Space Debris at GEO” Thesis. California Polytechnic State University, 2010.

[2] "Technical report on space debris" United Nations, New York, 1999.

[3 "NASA Orbital Debris FAQs." NASA Orbital Debris Program Office. Web. 06 Jan. 2011. <http://orbitaldebris.jsc.nasa.gov/faqs.html>.

[4] Abercromby Kira, J., Seitzer Patrocl, Rodriguez M. Heather, Barker S. Edwin, and Mark J. Matney. United States. International Astronautical Congress. "Surbey and Chase: A New Method of Observations for the Michigan Orbital Debris Surbey Telescope (MODEST).” Cong. Doc. 1st ed. Vol. B6. Print. Ser. 2.

[5] Ticha, Tichy M., Kocer M. “The Recovery as an Important Part of NEA Astrometric Follow-up" Icarus 159, 351-357, 2002.

[6] Milani, Andrea. “The Asteroid Identification Problem” Icarus 137, 269-292, 1999.

[7] Curtis, Howard D. Orbital Mechanics for Engineering Students. Amsterdam: Butterworth-Heinemann, 2010. Print.

[8] Vallado, David A. Fundamentals of Astrodynamics and Applications. New York: Springer, 2007. Print.

[9] Abercromby, K. J., Barker, E, S., Cowardin, H. M., Matney, M. J., Parr-Thumm, T. L. "Michigan Orbital Debris Survey Telescope (MODEST) Observations of the Geosynchronous Orbital Debris Environment.” NASA/TP-2010-under review. 
[10] Agapov, V., Biryukov, V., Kiladze, R., Molotov, I., Rumyantsev, V., Sochilina, A., Titenko V. "Faint GEO Objects Search and Orbital Analysis" Proceedings of the 4th European Conference on Space Debris (ESA SP-587). 18-20 April 2005, ESA/ESOC, Darmstadt, Germany. Editor: D. Danesy., p.153. The SAO/NASA Astrophysics Data System.

[11] National Research Council. Orbital Debris: A Technical Assessment. Washington, D.C.: National Academy Press. 1995.

[12] Abercromby, K. J., Barker, E, S., Rodriguez, H., Seitzer, P. “Optical Studies of Orbital Debris at GEO Using Two Telescopes."

[13] Carpino, M. Chesley, S, R. Milani, A. "Error statistics of asteroid optical astrometric observations" Icarus 166 (2003) 248-270. 


\section{Appendix A: Code}

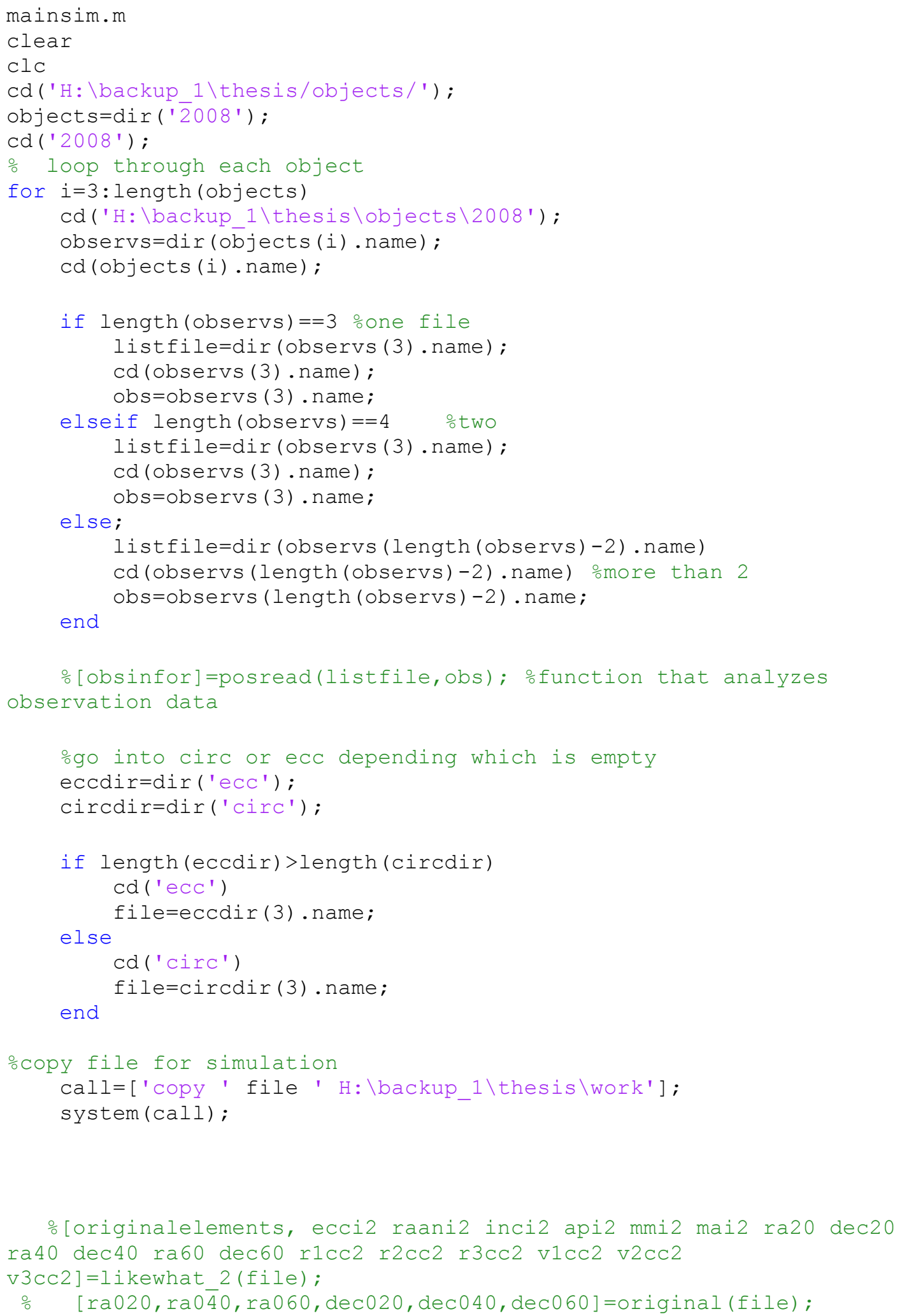




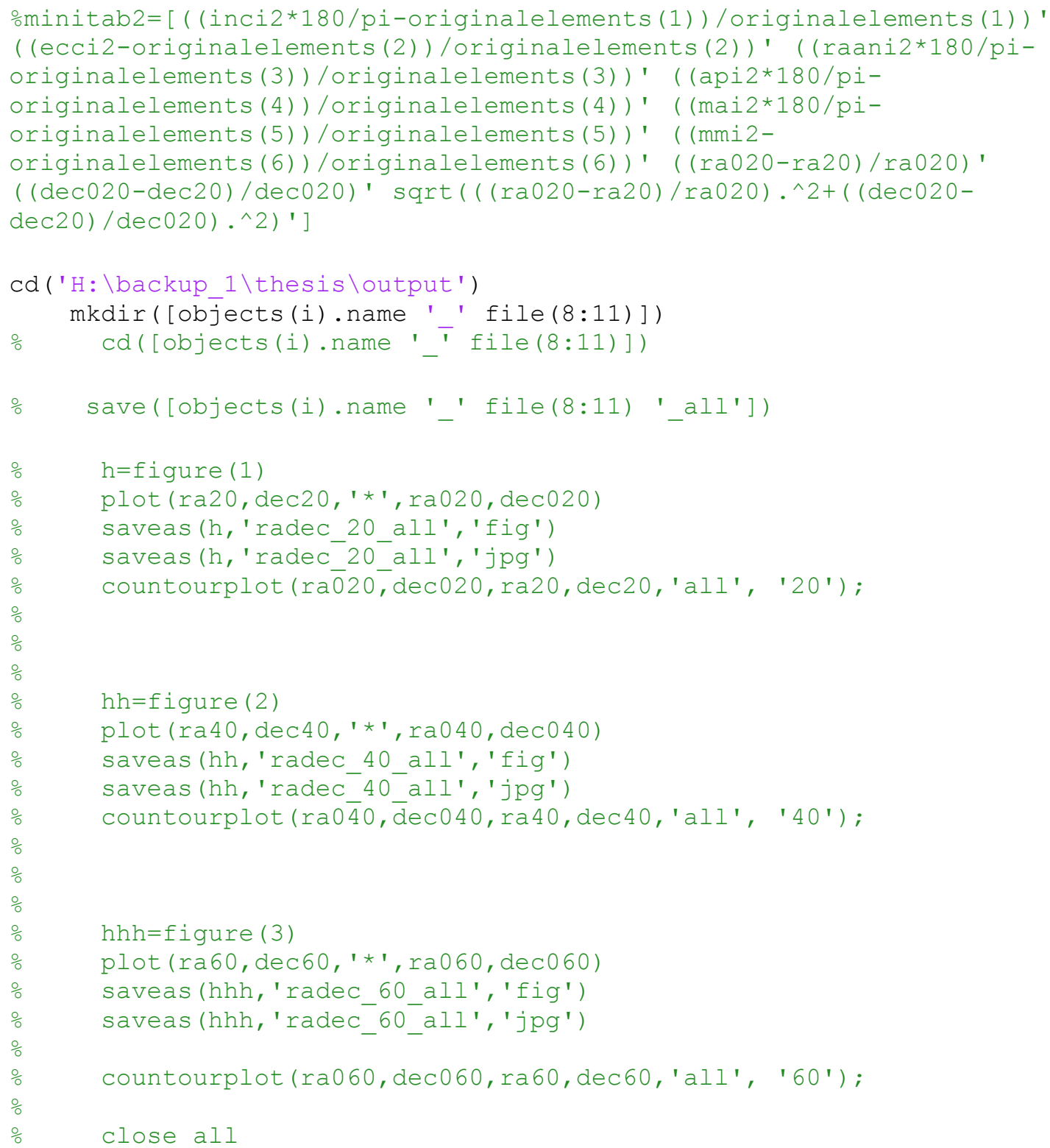

[originalelements, ecci raani inci api mmi mai ra20 dec20 ra40 dec40 ra60 dec60 rlcc r2cc r3cc v1cc v2cC v3cc]=sim_iterate(file); $[\mathrm{ra} 020, \mathrm{ra} 040, \mathrm{ra} 060, \operatorname{dec} 020, \operatorname{dec} 040, \operatorname{dec} 060]=0 r i g i n a \bar{l}$ (file);

minitab $=[($ (inci*180/pi-originalelements (1) )/originalelements (1)) ' ( (ecci-originalelements (2)) /originalelements (2))' ((raani*180/pioriginalelements (3))/originalelements (3))' ((api*180/pioriginalelements (4))/originalelements(4))' ((mai*180/pioriginalelements (5)) /originalelements(5))' ((mmioriginalelements (6)) /originalelements (6))' ((ra020-ra20)/ra020)' $(($ dec020-dec20)/dec020)' sqrt ( ( ra020-ra20)/ra020) .^2+((dec020dec20)/(dec020).^2)']

cd ('H: \backup_1 1thesis loutput')

cd([objects(i).name '-'file(8:11)])

save ([objects(i).name ', file(8:11) '_rand']) 


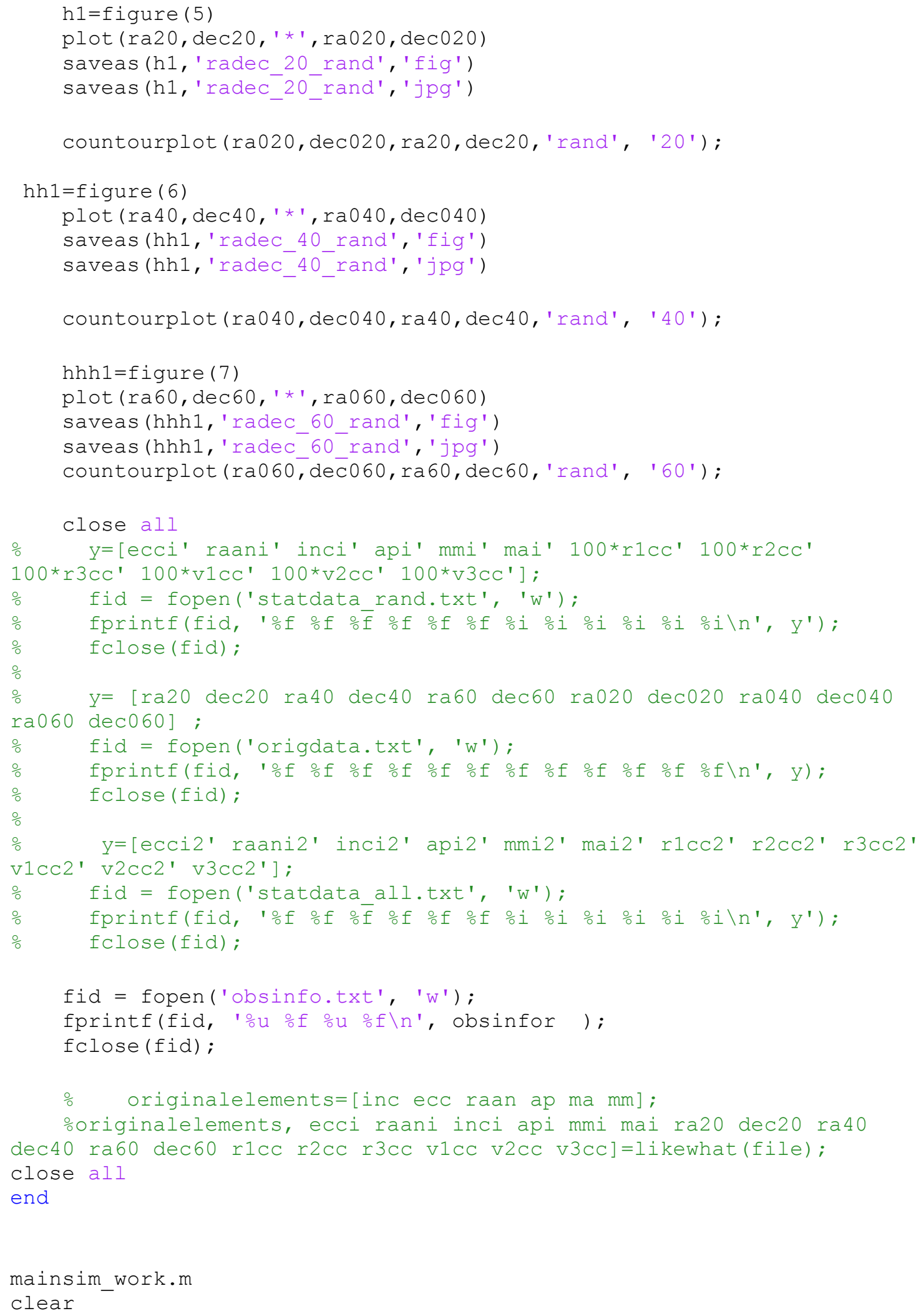




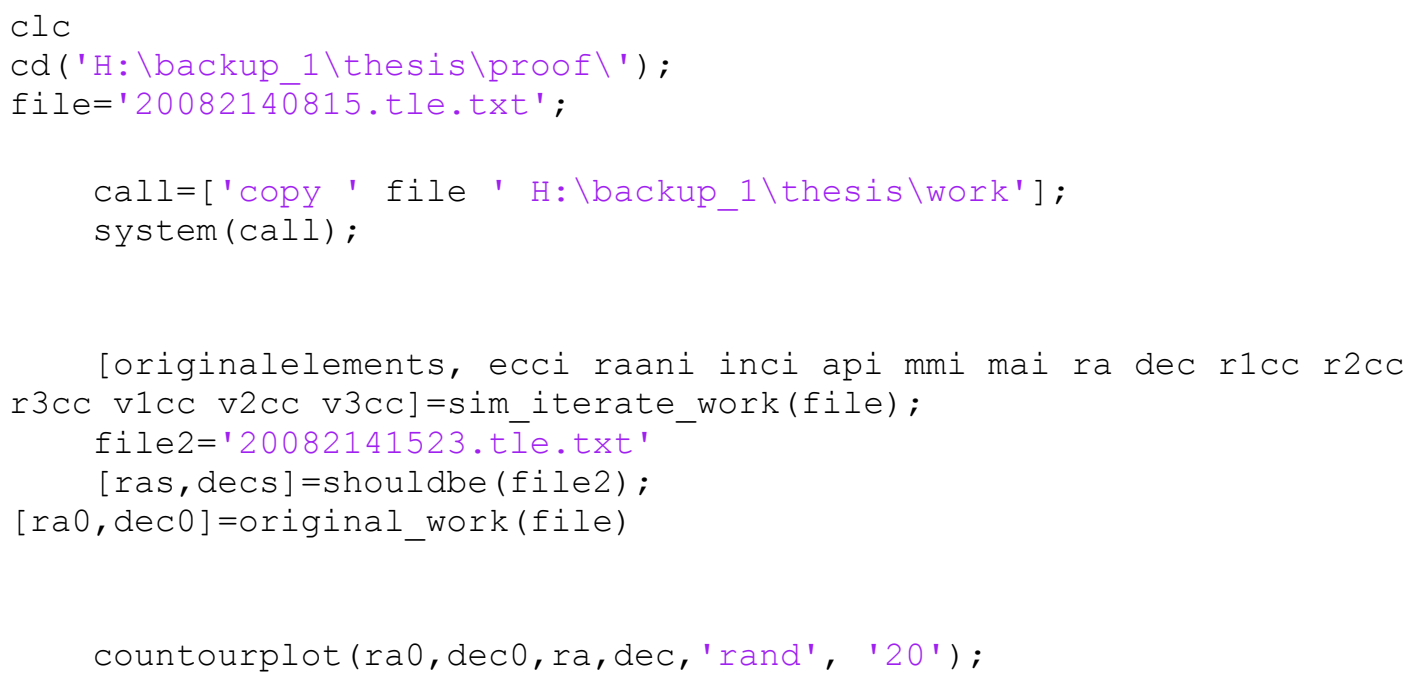

\section{bancompiler.m}

clear

clc

cd ('H: \backup_1 \thesis \work');

file='20082200312.tle.txt'

[originalelements, ecci raani inci api mmi mai ra20 dec20 ra40

dec40 ra60 dec60 rlcc r2cc r3cc v1cc v2cc v3cc]=sim_iterate(file);

[ra020,ra040,ra060, dec020, dec040, dec060]=original (file);

countourplot (ra020, dec020,ra20, dec20, 'rand', '20') ;

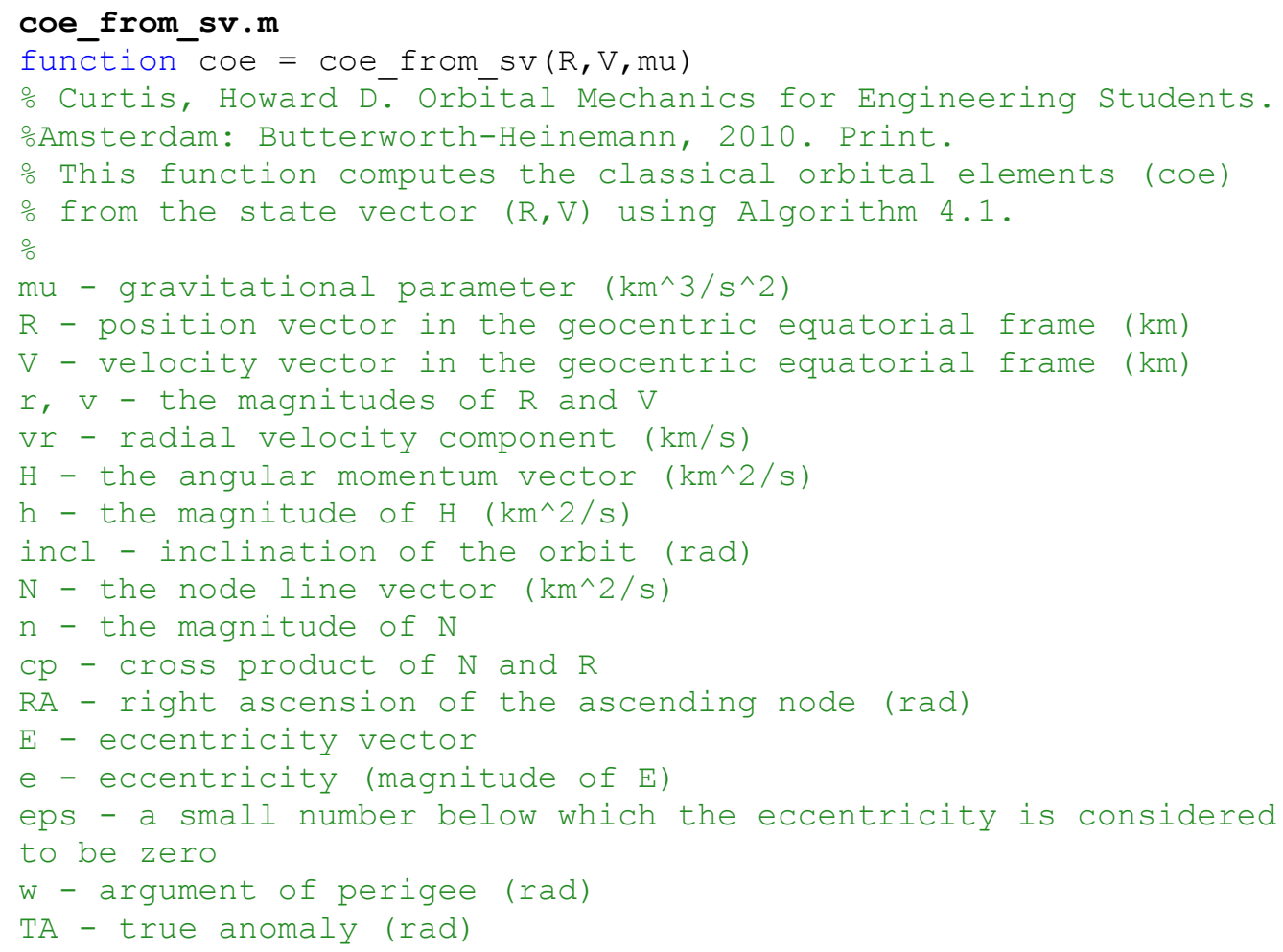




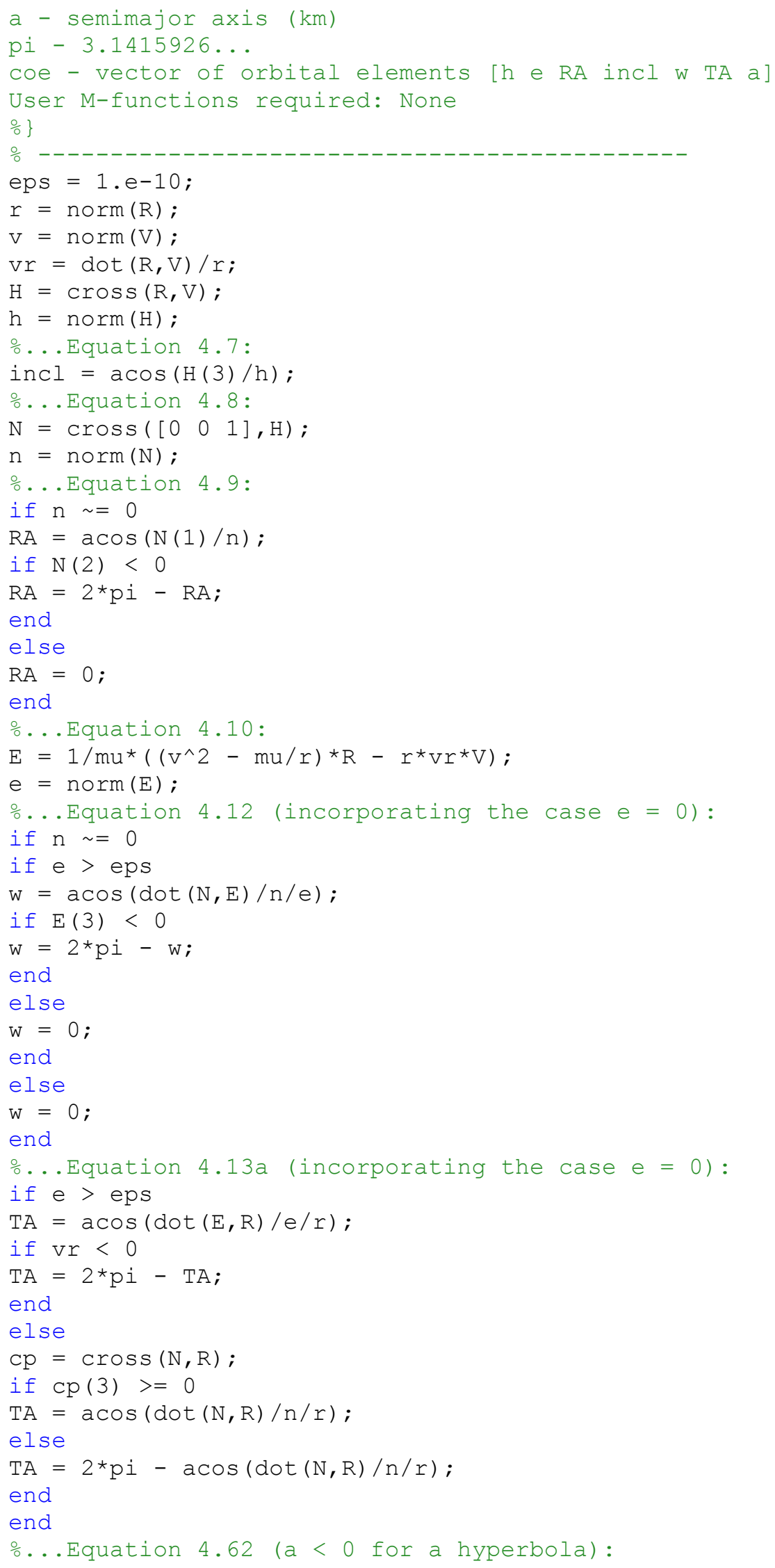




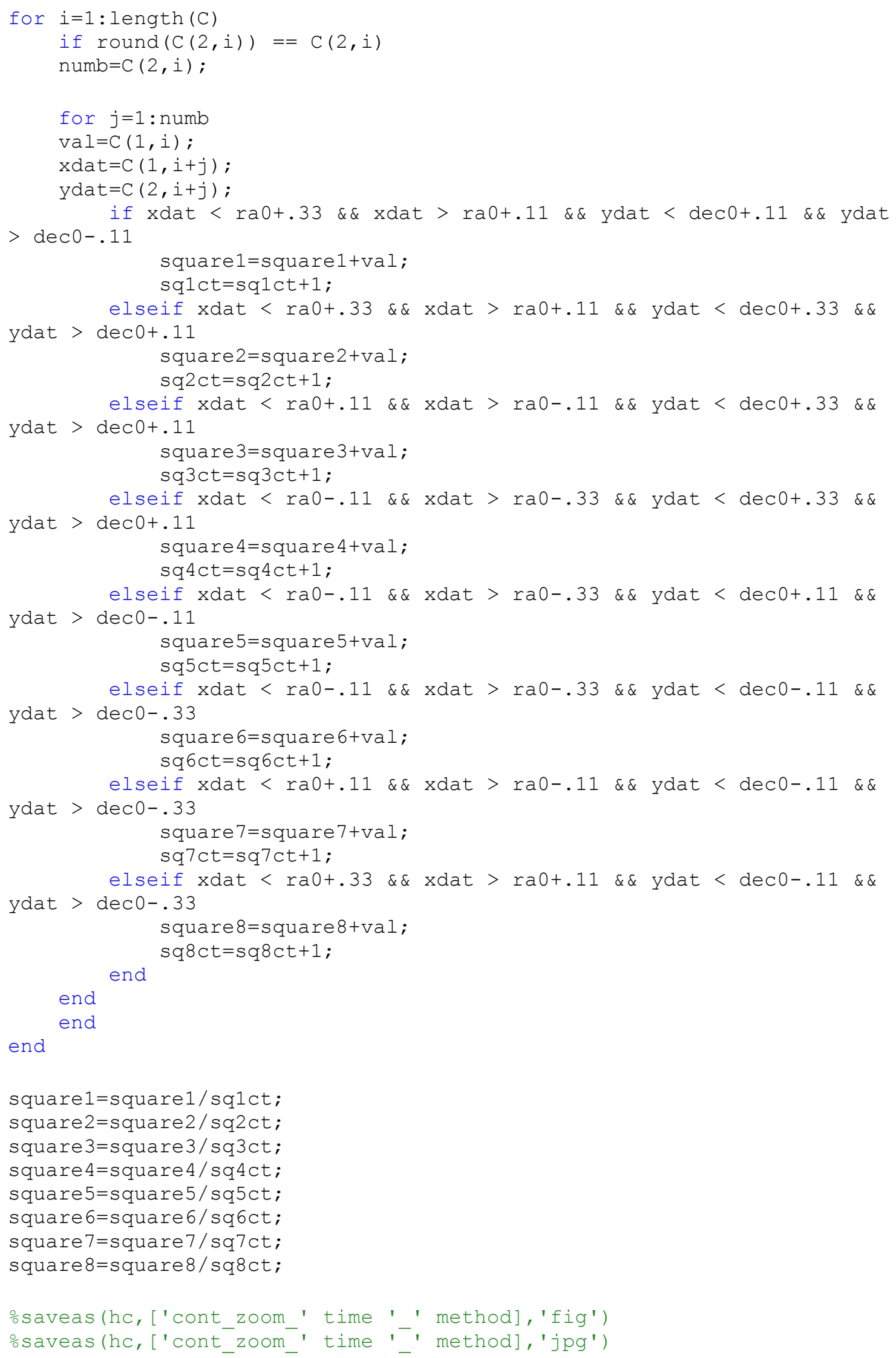




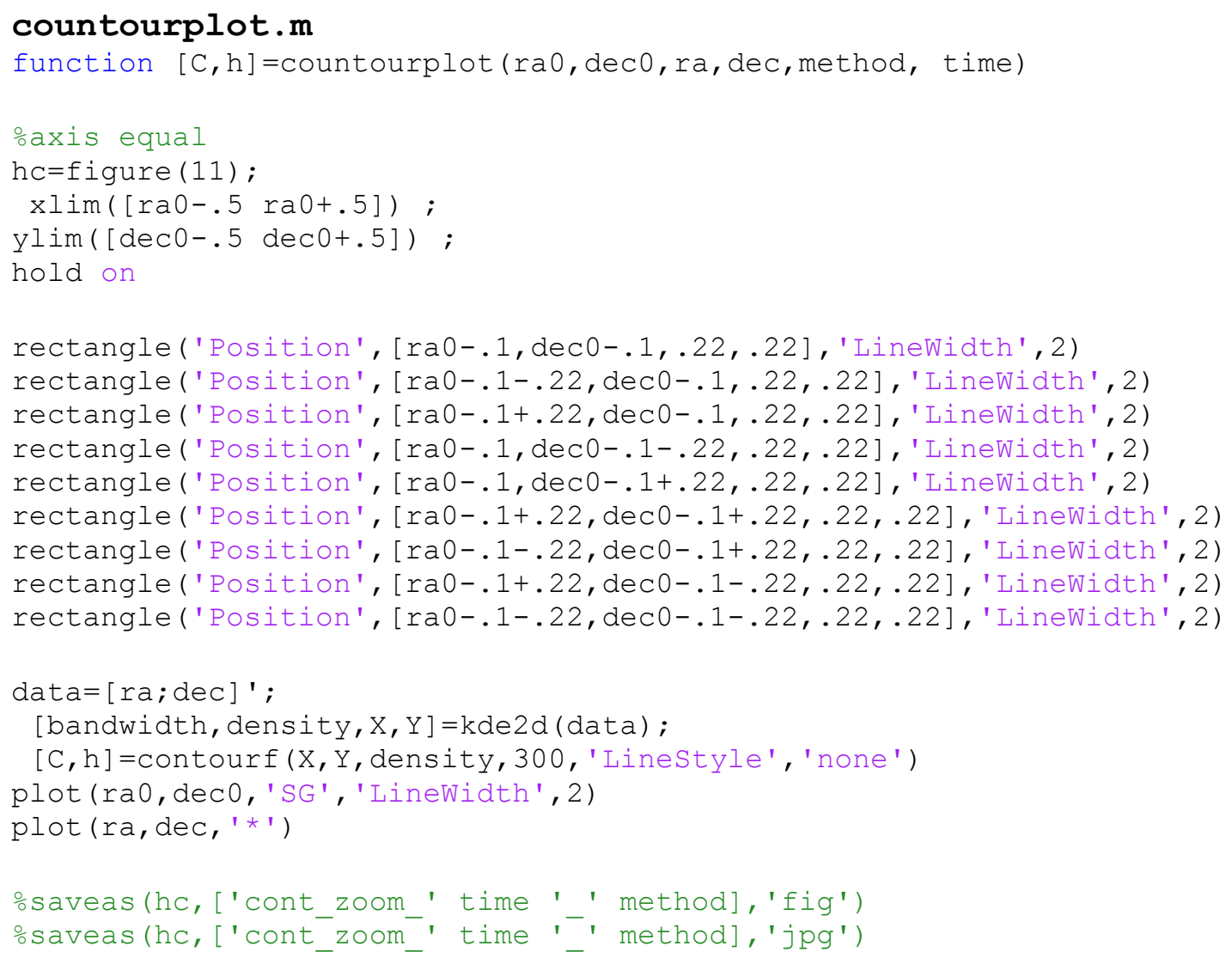

\section{densecounter.m}

clear

$\mathrm{clc}$

cd ('H: \backup 1\thesis/output/') ;

outputs=dir (' $\overline{2} 007$ ');

cd ('2007');

count $1=0$;

count $2=0$;

$\operatorname{count} 3=0$

count $4=0$;

count $5=0$;

count $6=0$;

count $7=0$;

count $8=0$;

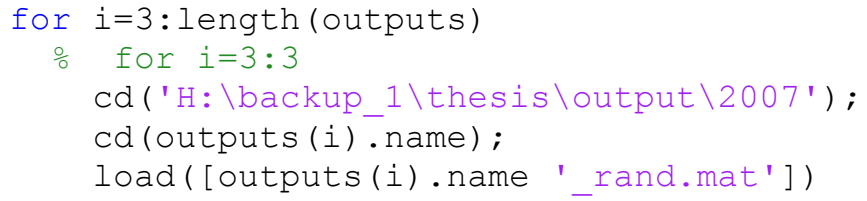

[square1, square2, square3, square4, square 5, square6, square7, square 8] =count density (ra020, dec020, ra20, dec20)

$[C, I]=\max ([$ square1 square2 square3 square4 square5 square6 square7 square 8] ) 


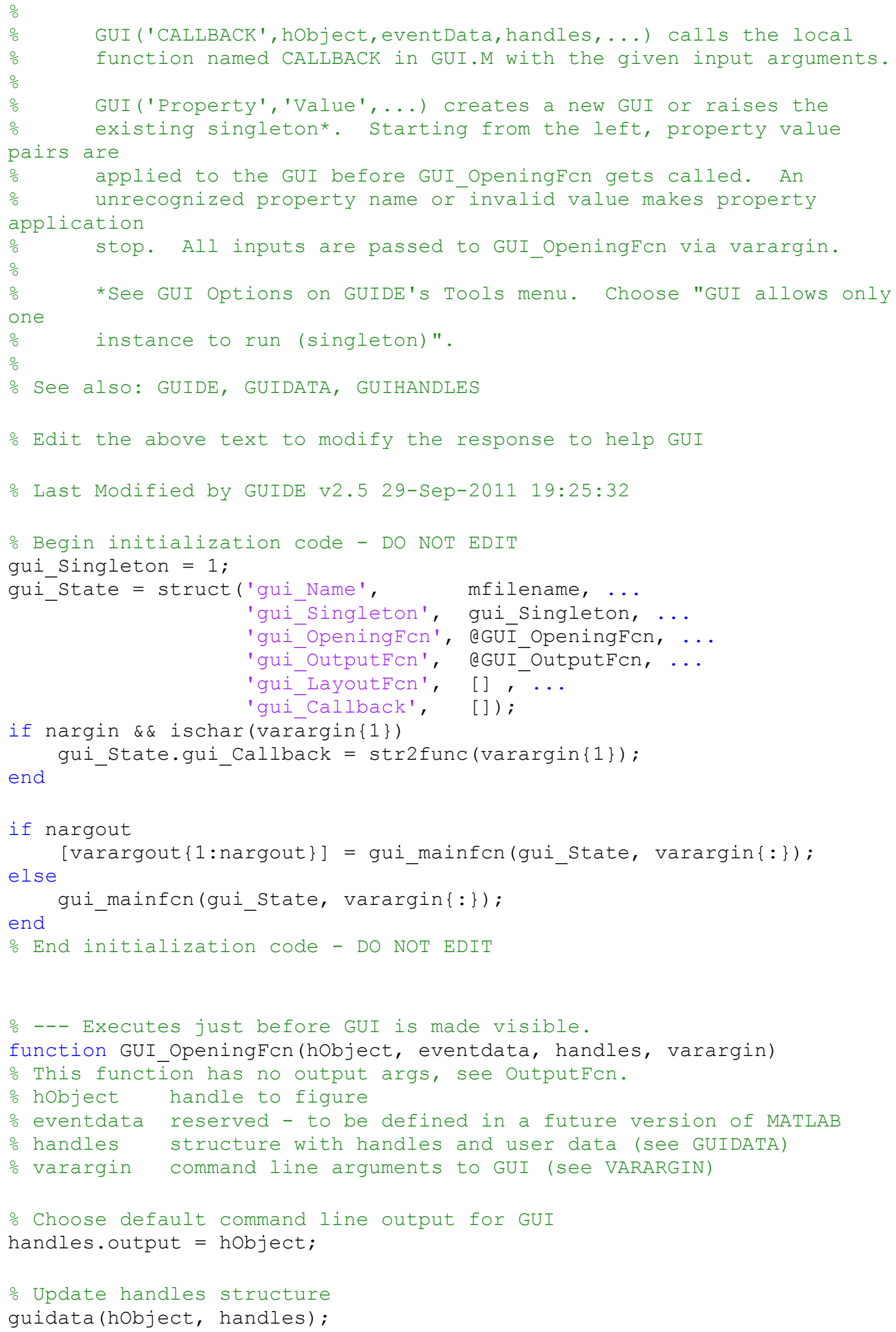


\% UIWAIT makes GUI wait for user response (see UIRESUME)

o uiwait (handles.figurel);

\% --- Outputs from this function are returned to the command line.

function varargout = GUI OutputFcn (hobject, eventdata, handles)

\% varargout cell array for returning output args (see VARARGOUT);

\% hobject handle to figure

o eventdata reserved - to be defined in a future version of MATLAB

o handles structure with handles and user data (see GUIDATA)

\% Get default command line output from handles structure

varargout $\{1\}=$ handles.output;
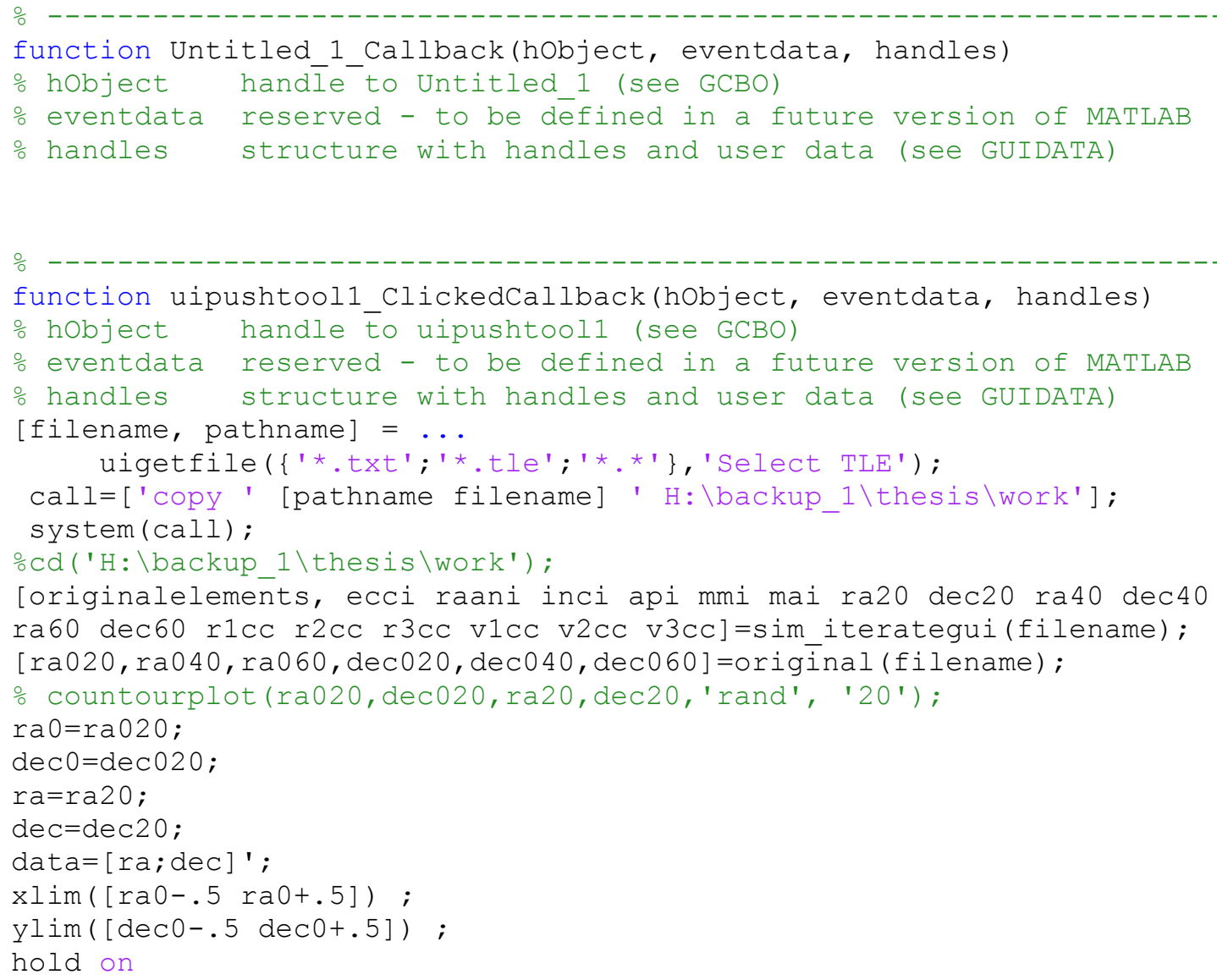


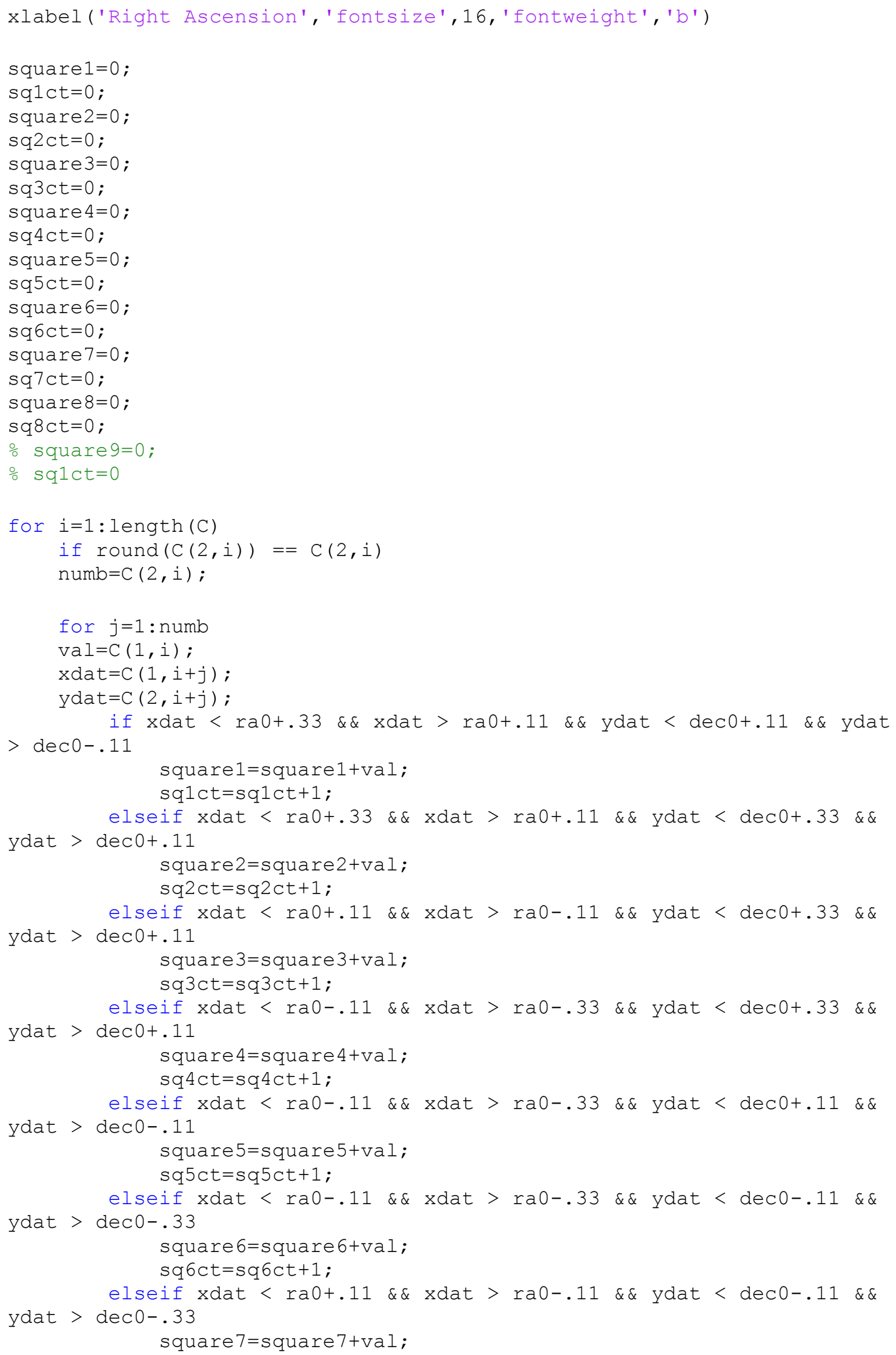




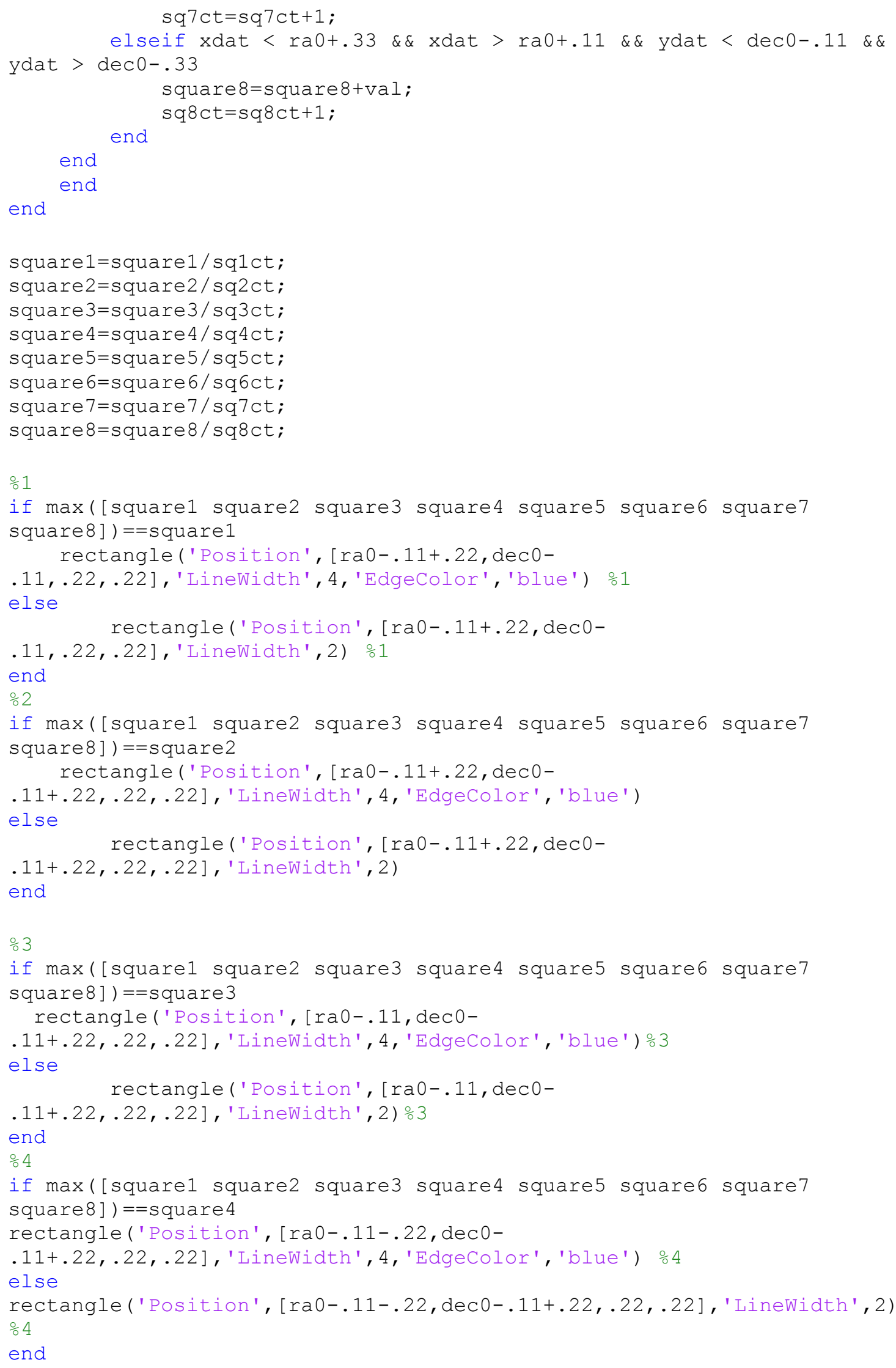




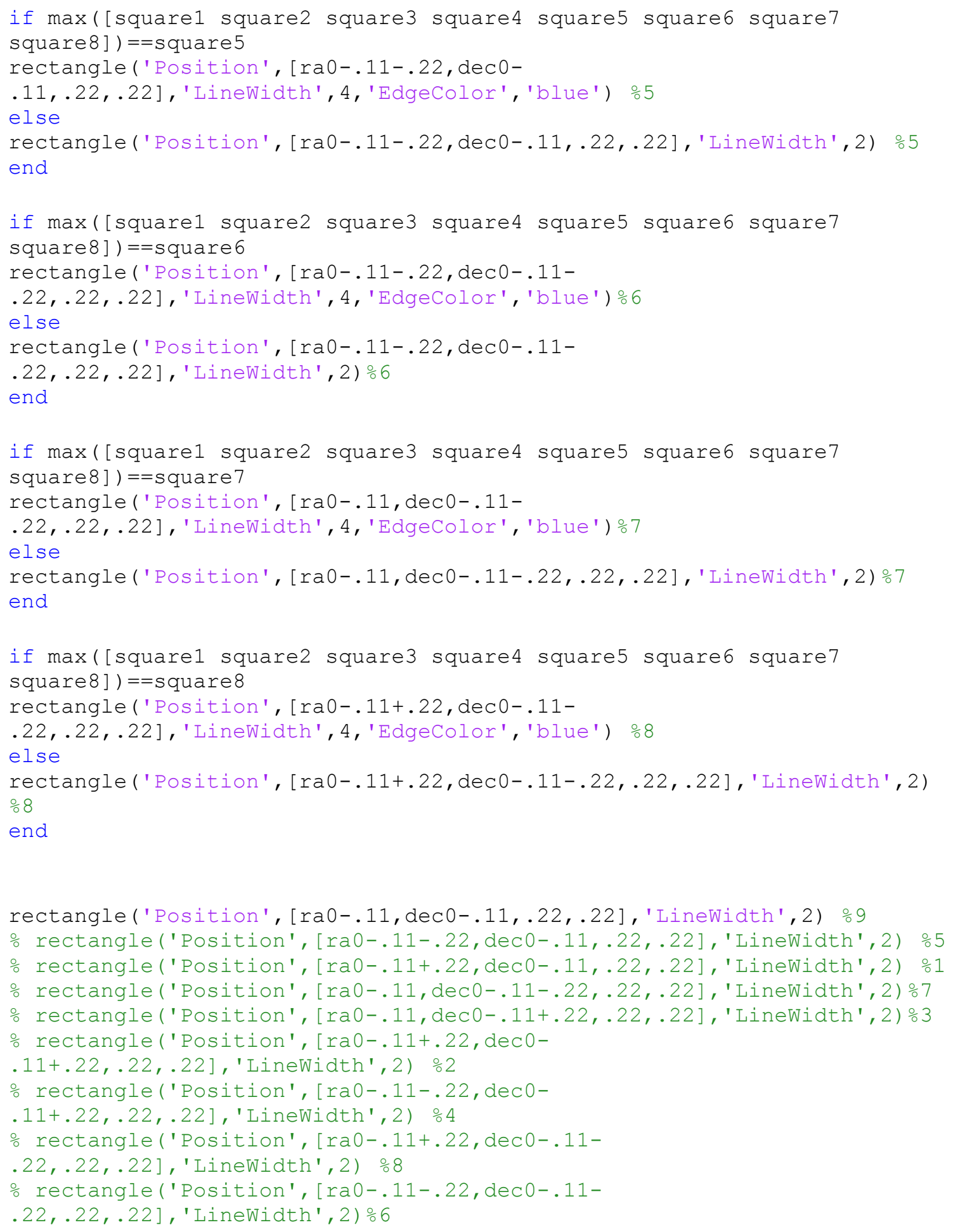

\section{kde2d.m}

function [bandwidth, density, X,Y] $=k \operatorname{de} 2 d$ (data, n, MIN_XY, MAX_XY)

\% fast and accurate state-of-the-art

o bivariate kernel density estimator

o with diagonal bandwidth matrix. 


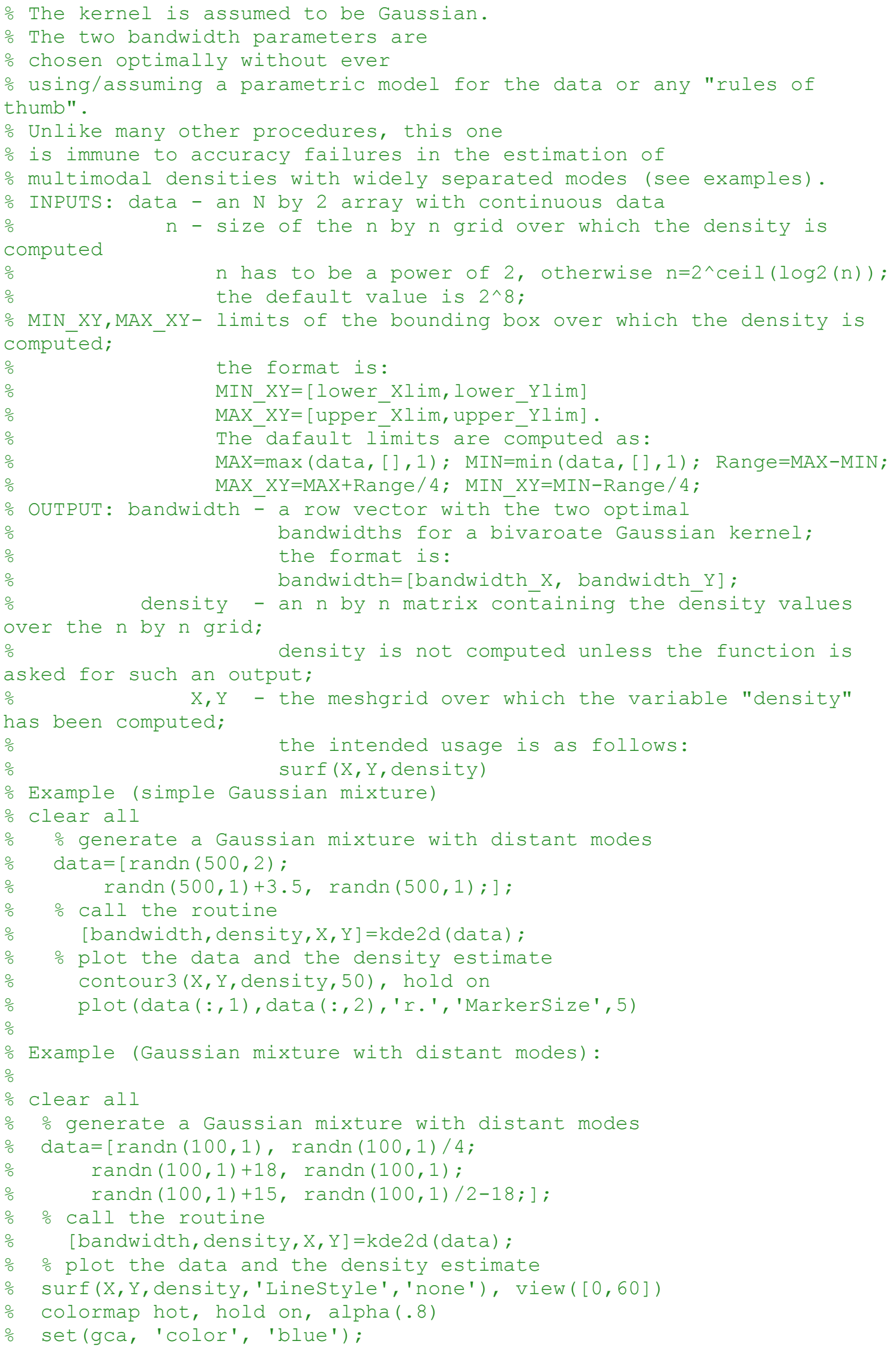



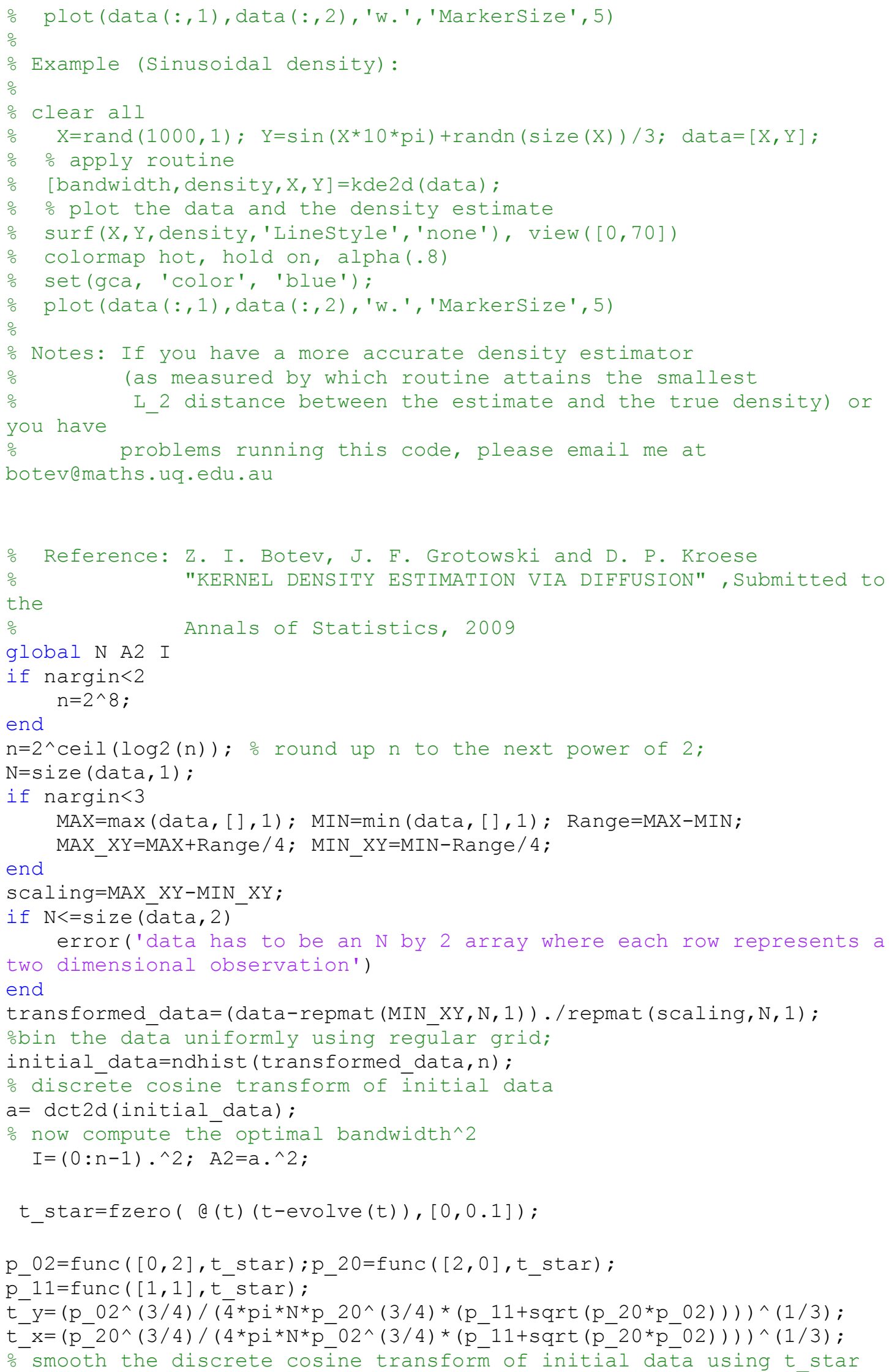


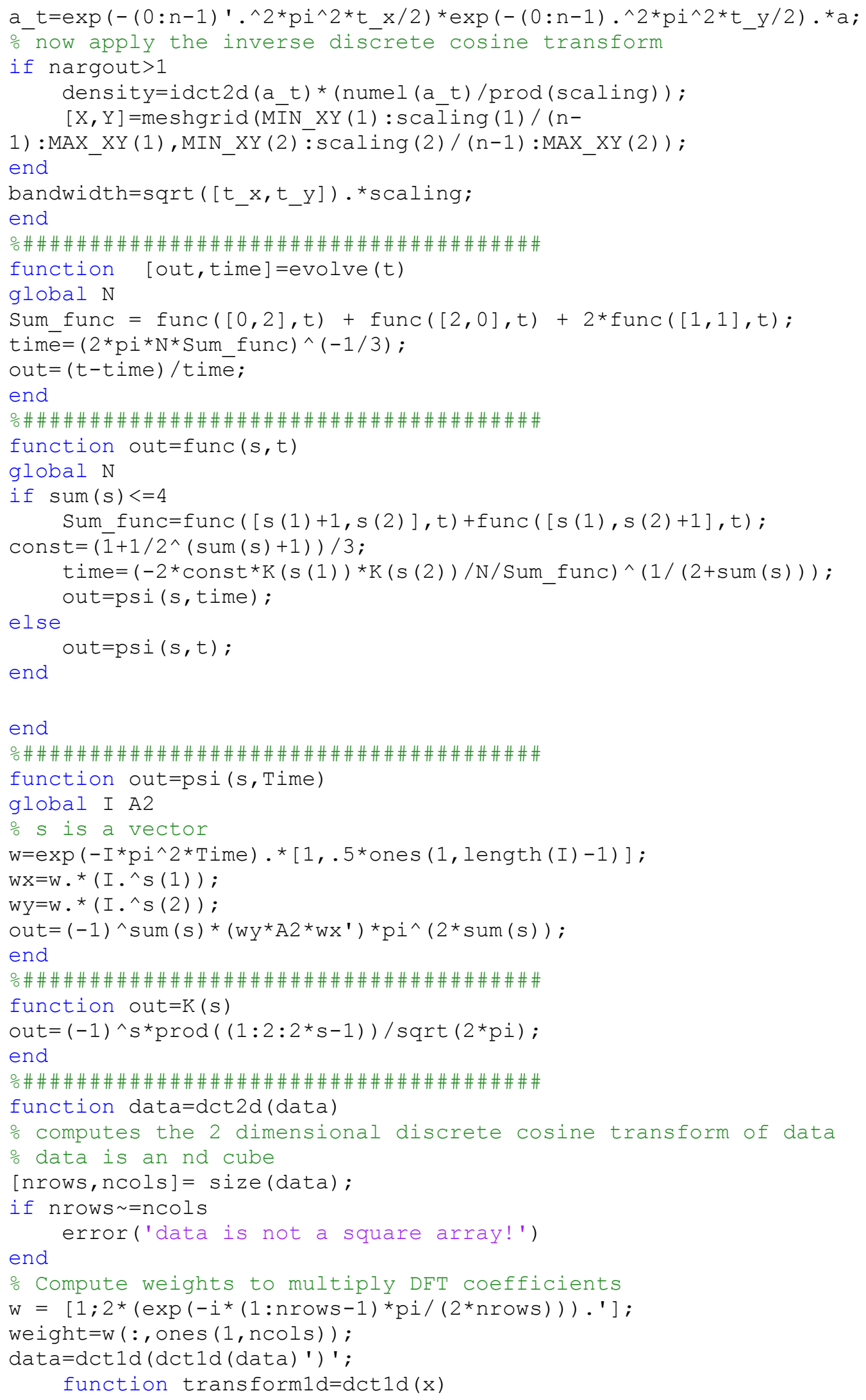




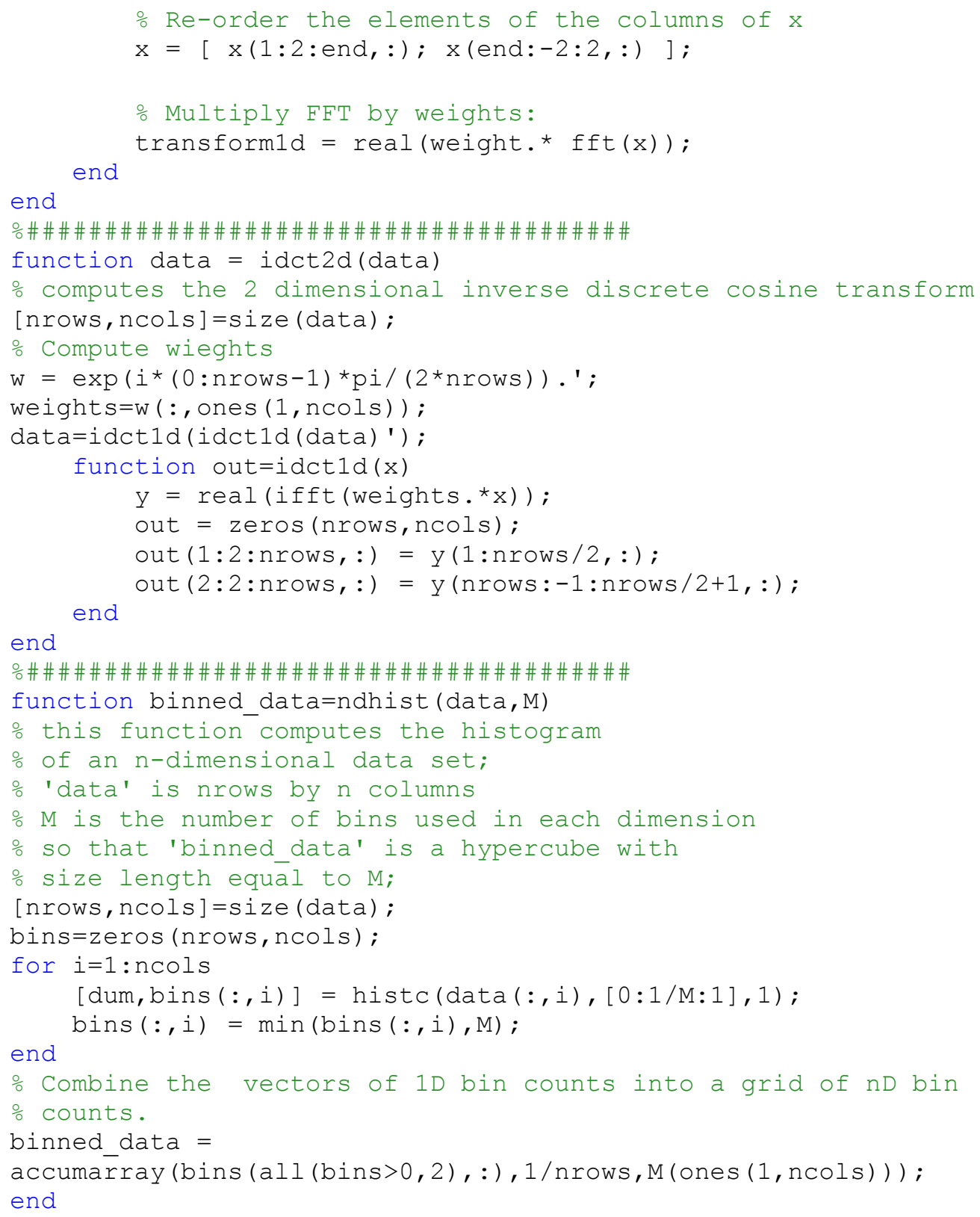

\section{kepler_E.m}

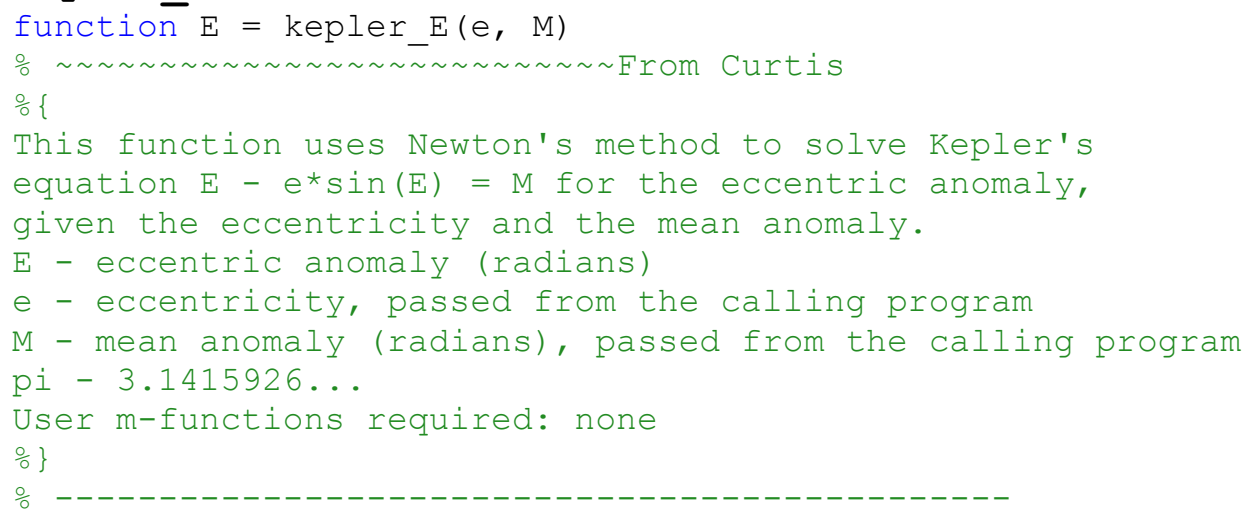




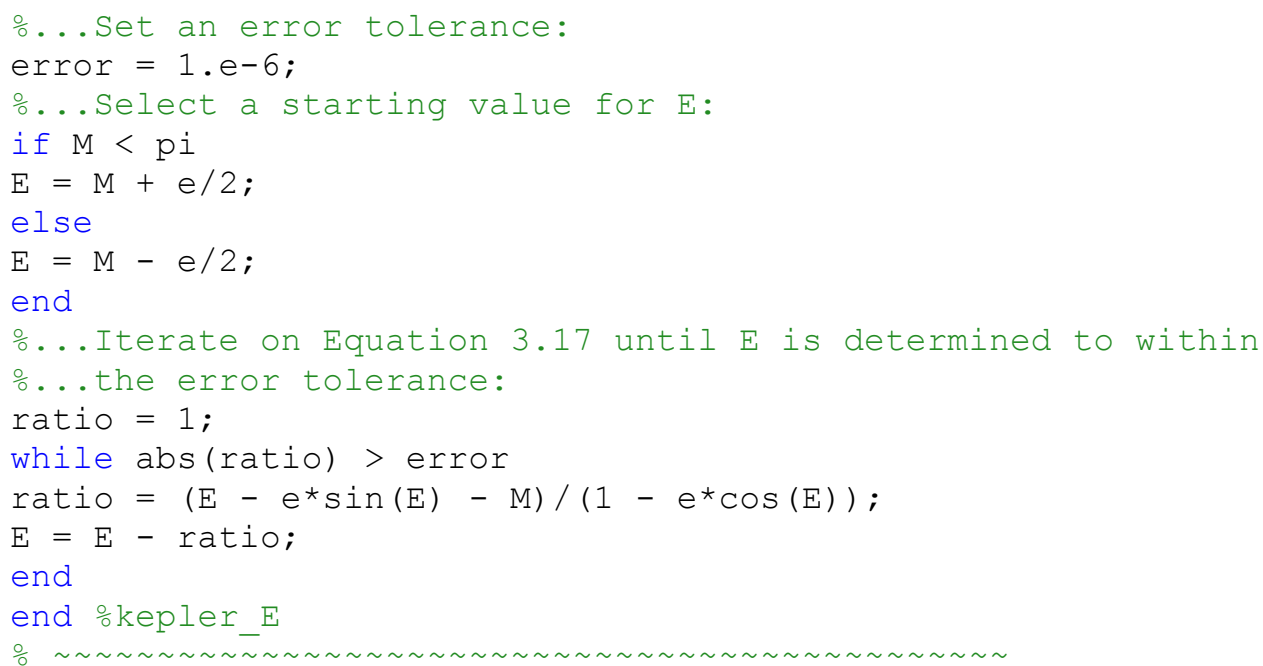

\section{sim iterate.m}

function [originalelements, ecci raani inci api mmi mai ra20 dec20 ra40 dec40 ra60 dec60 r1cc r2cc r3cc v1cC v2cC v3cc]=likewhat(file) 


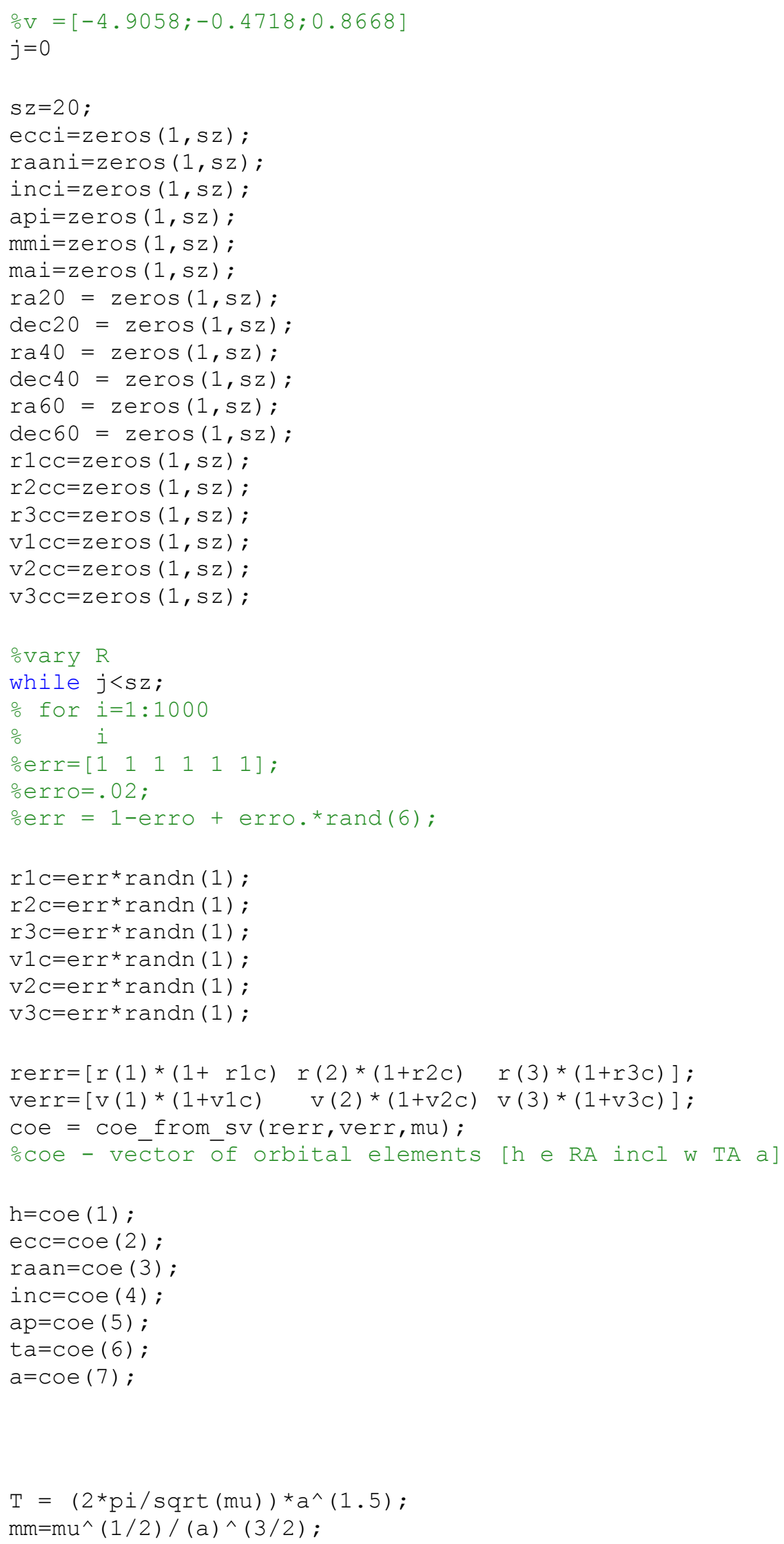




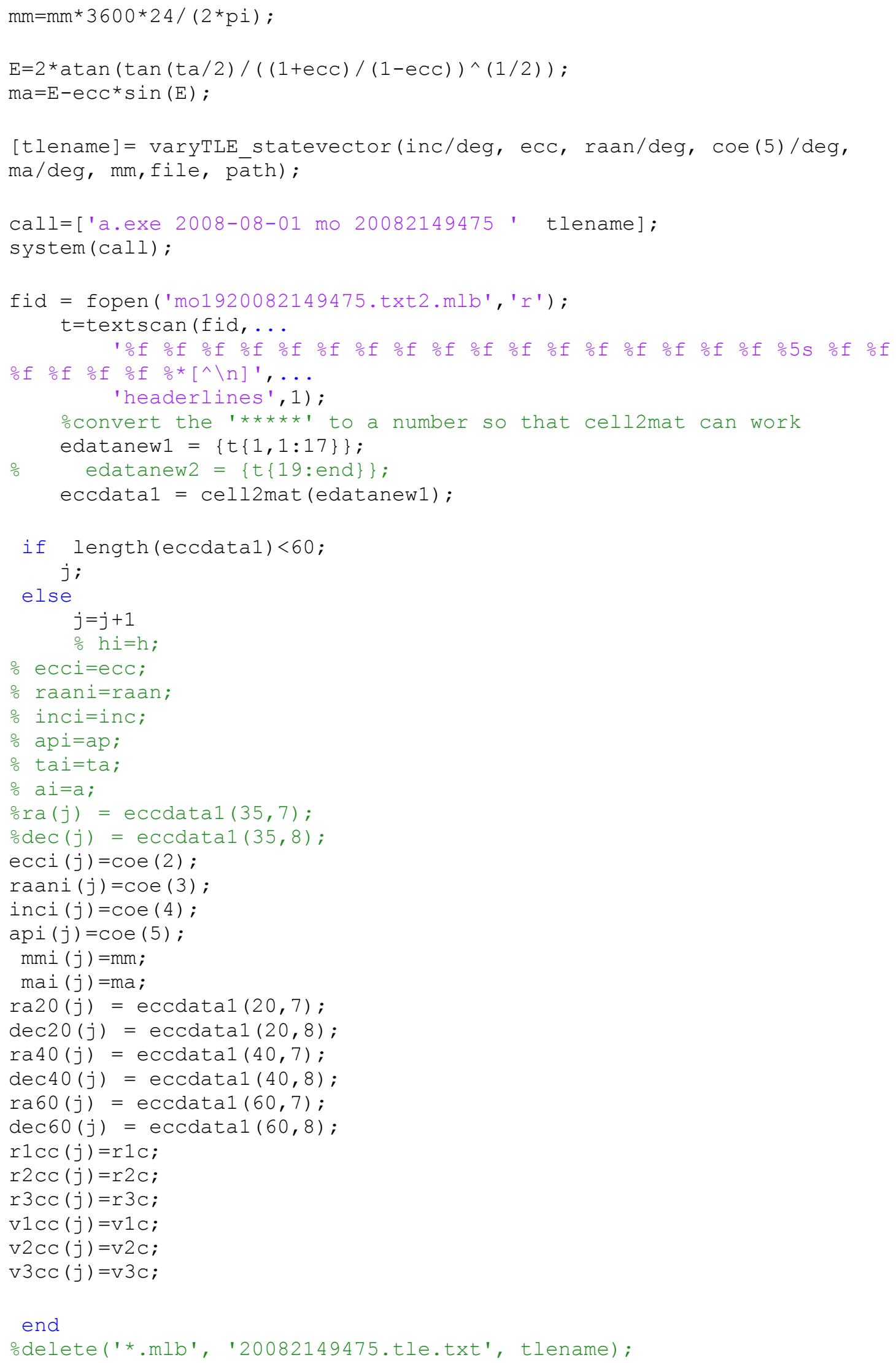


end

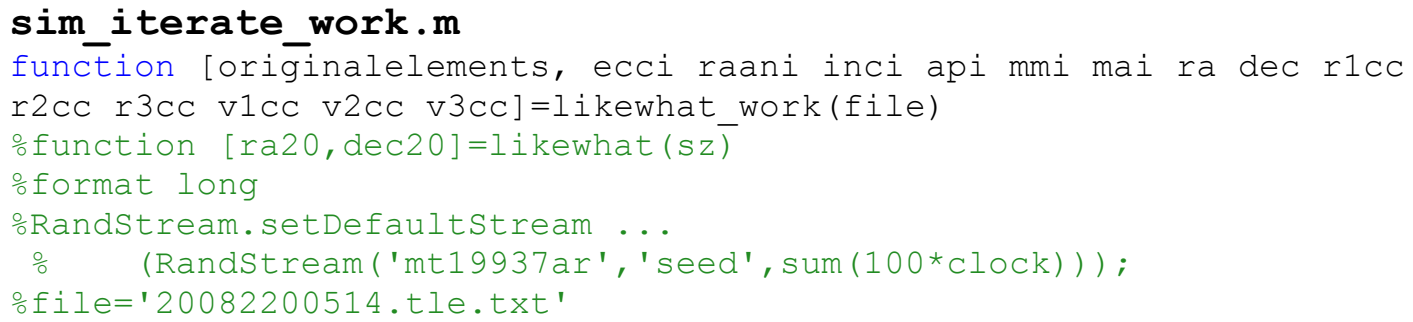




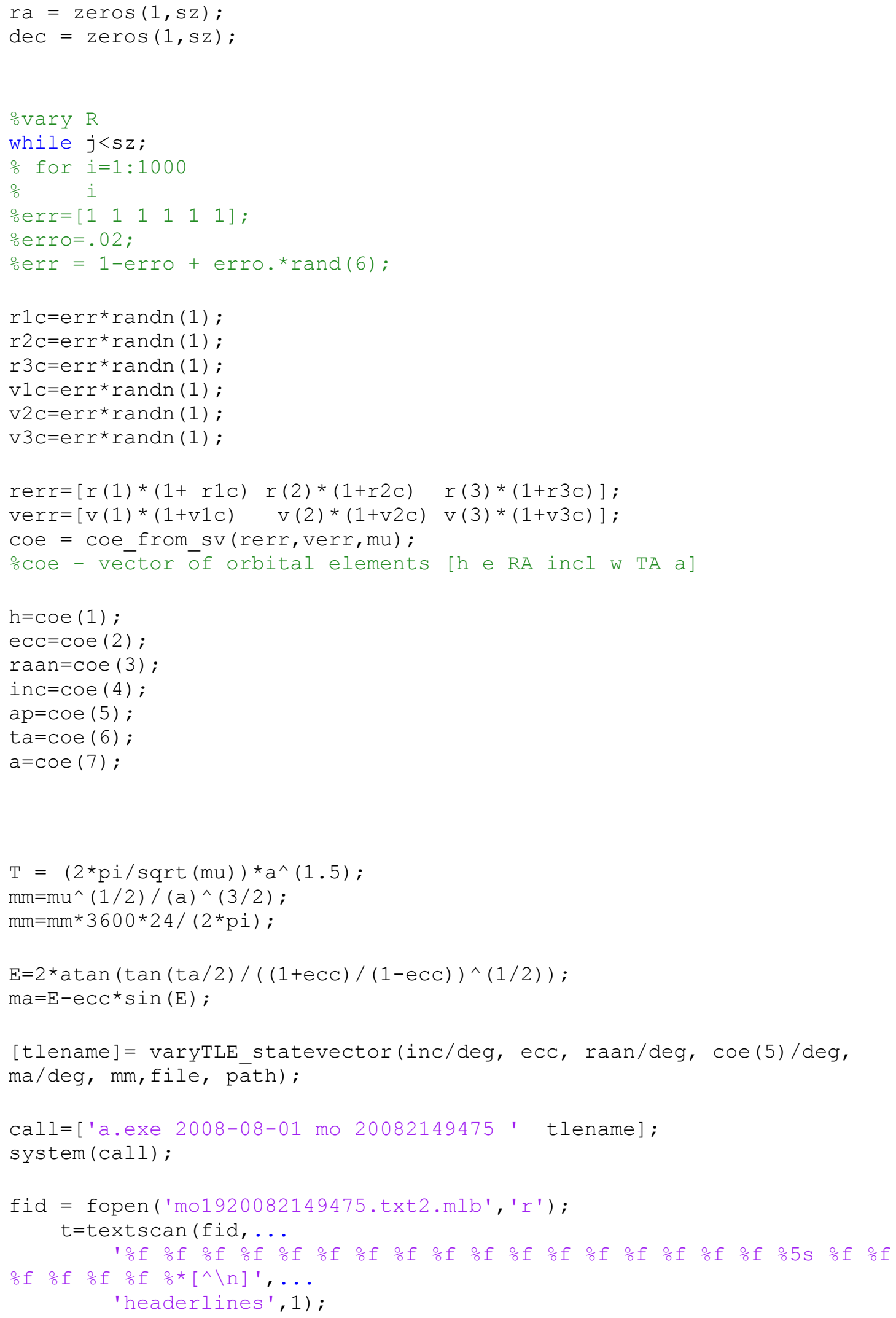




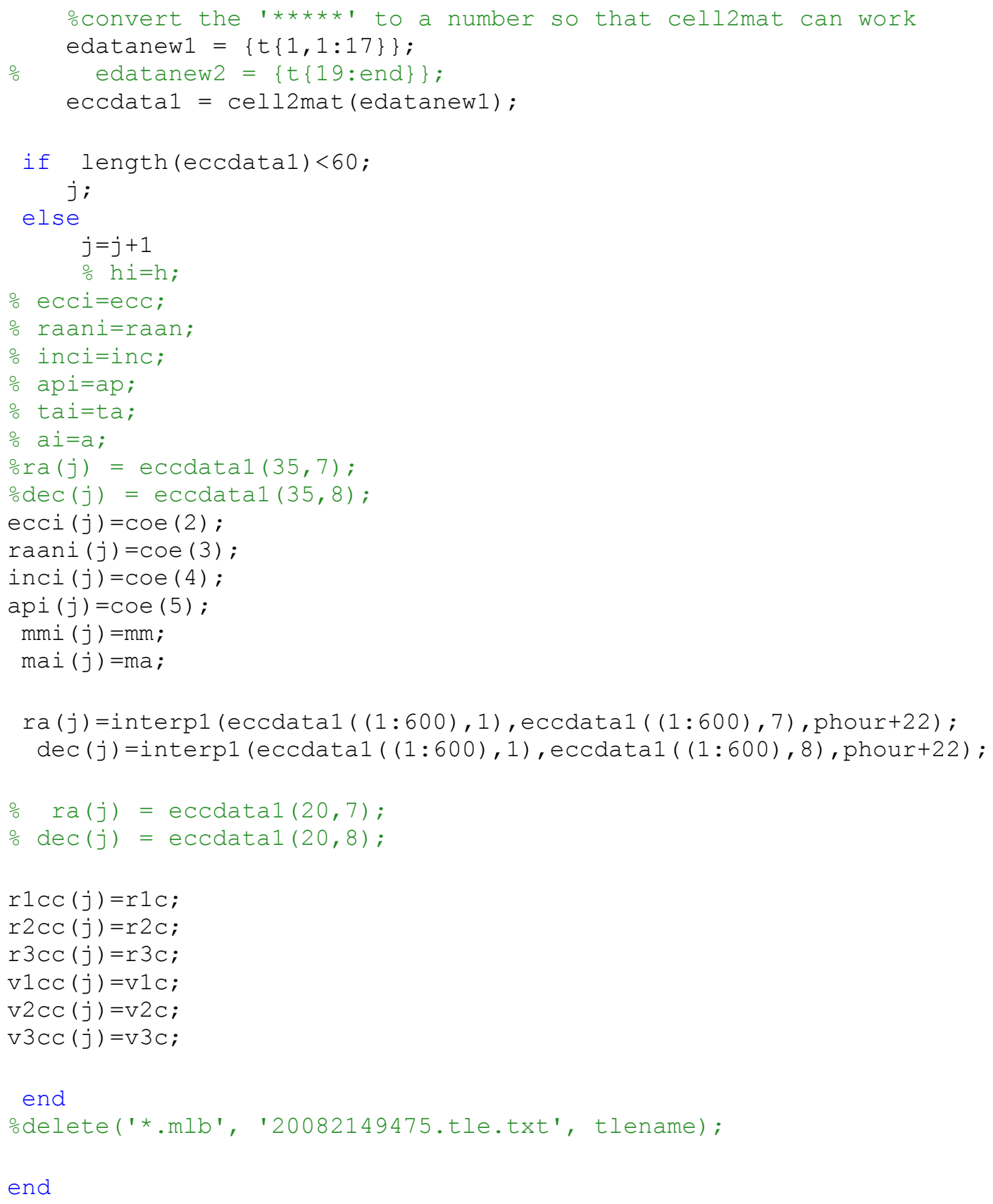




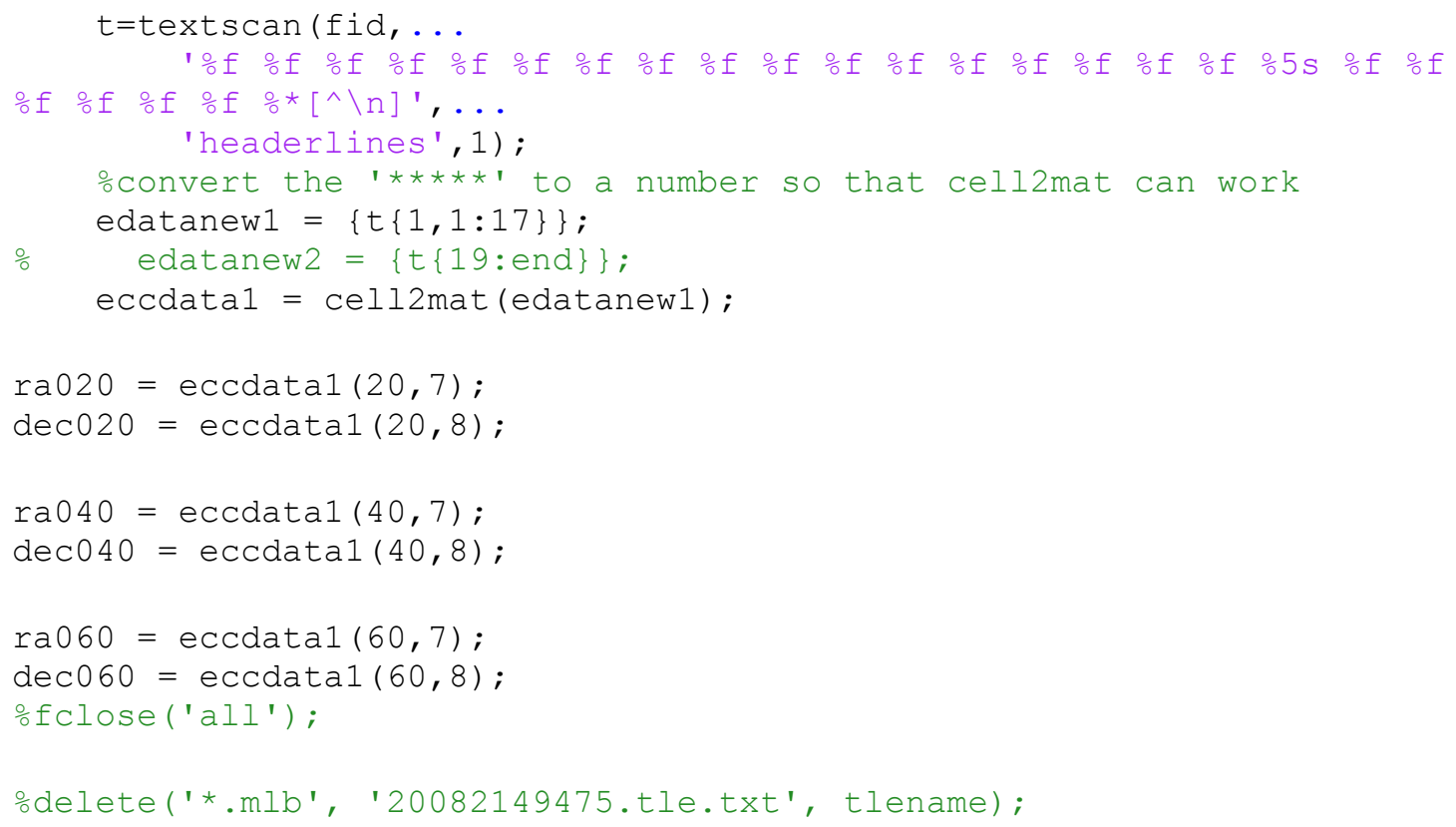

\section{original_work.m}

function [ra0, dec0]=original_work (file)

opath=['c: \cygwin\tmp' ];

ocd (path);

phour=1.7477;

call=['a.exe 2008-08-01 mo 20082149475 'file];

system (call);

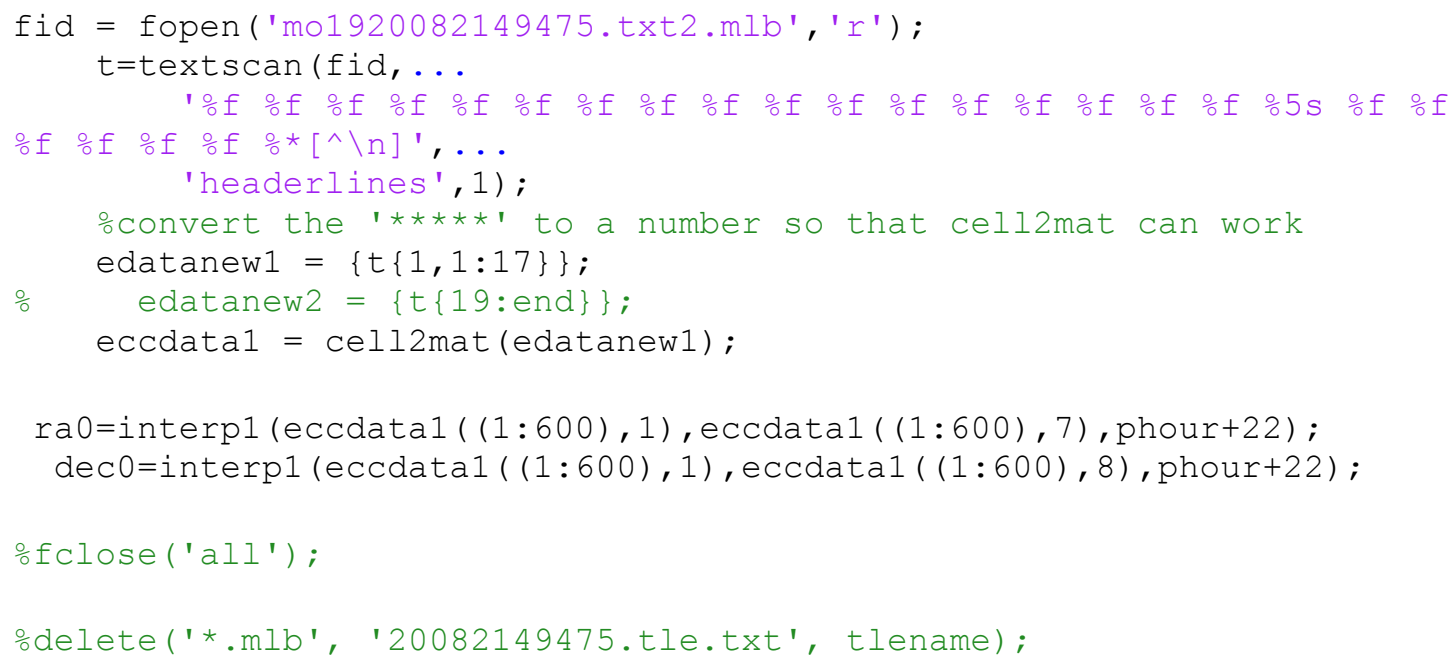

\section{posread.m}

function [obsinfor]=posread (listfile, obs)

ocd ( '/home/daniel/Desktop/backup/thesis/objects/' )

\% fid = fopen('pos20082170912.txt','r'); 


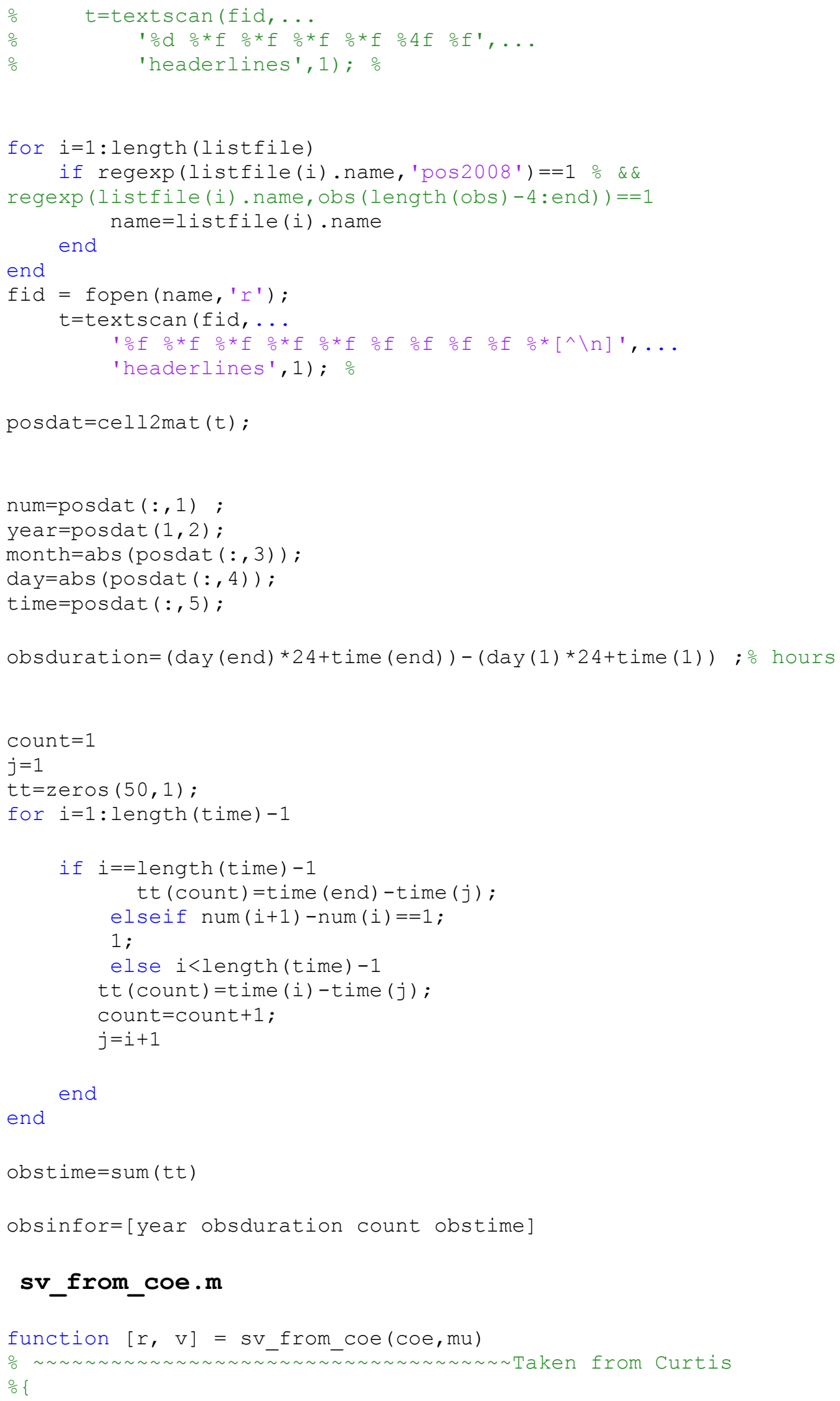




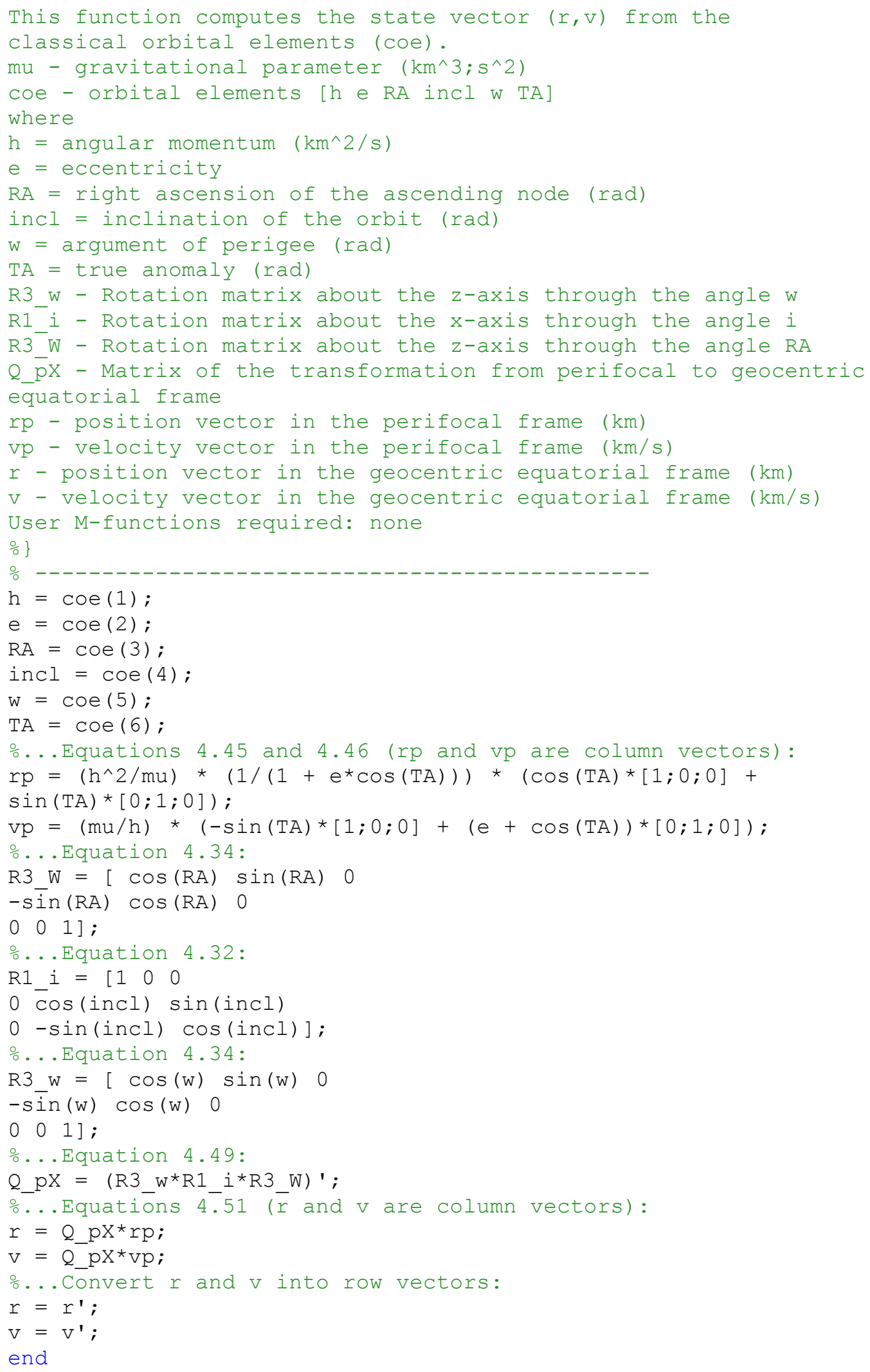

varYTLE.m 


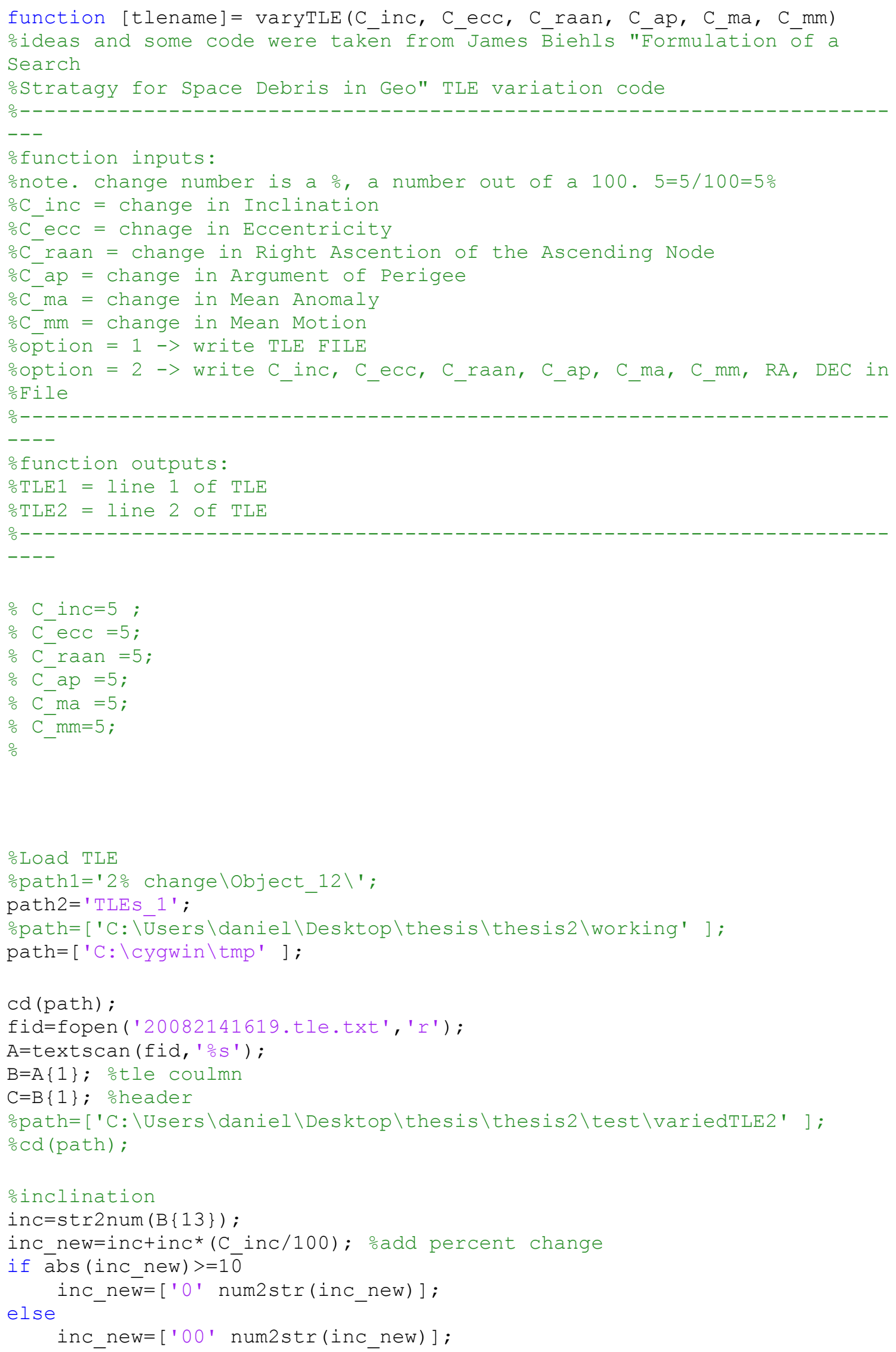




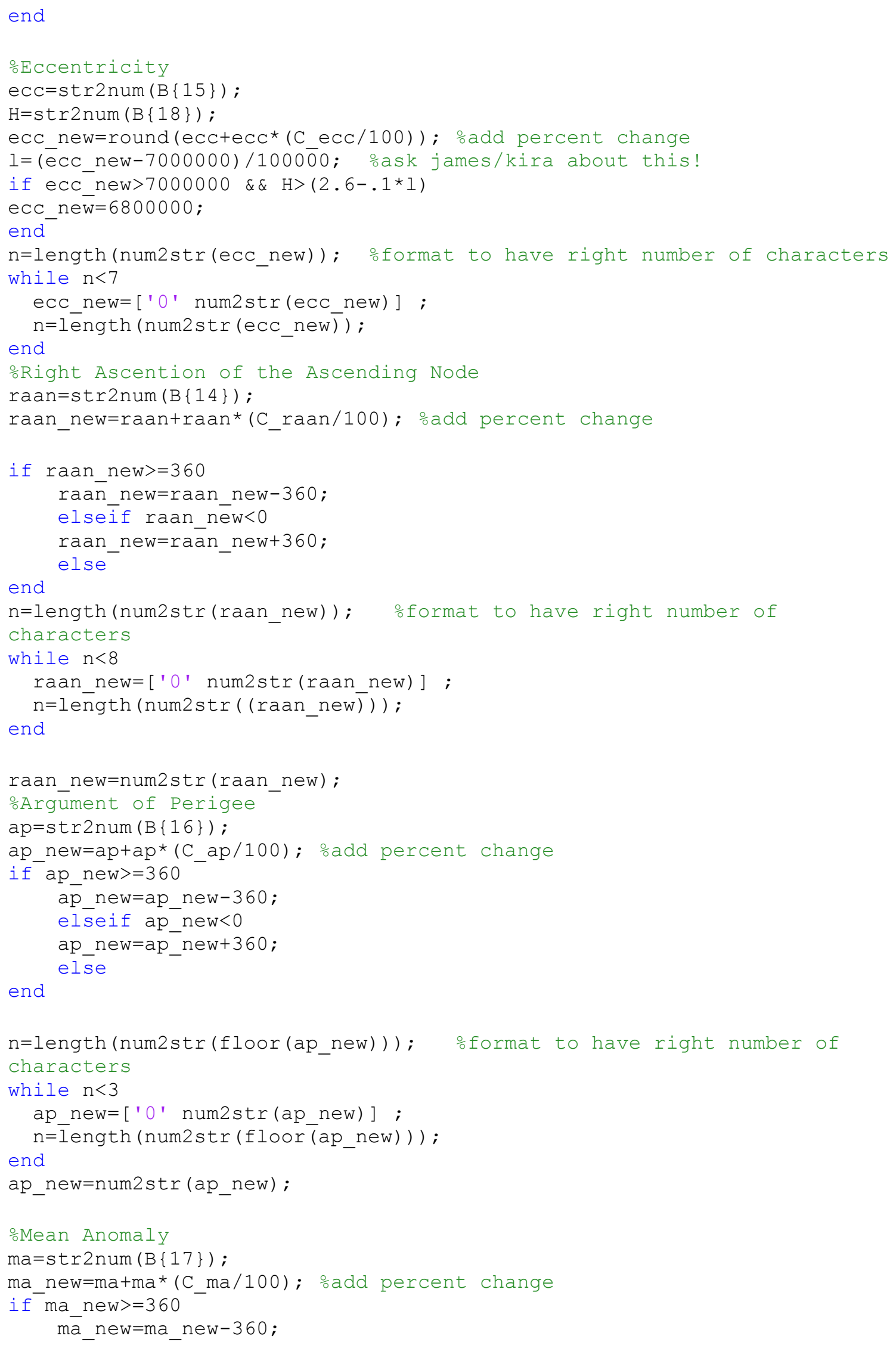




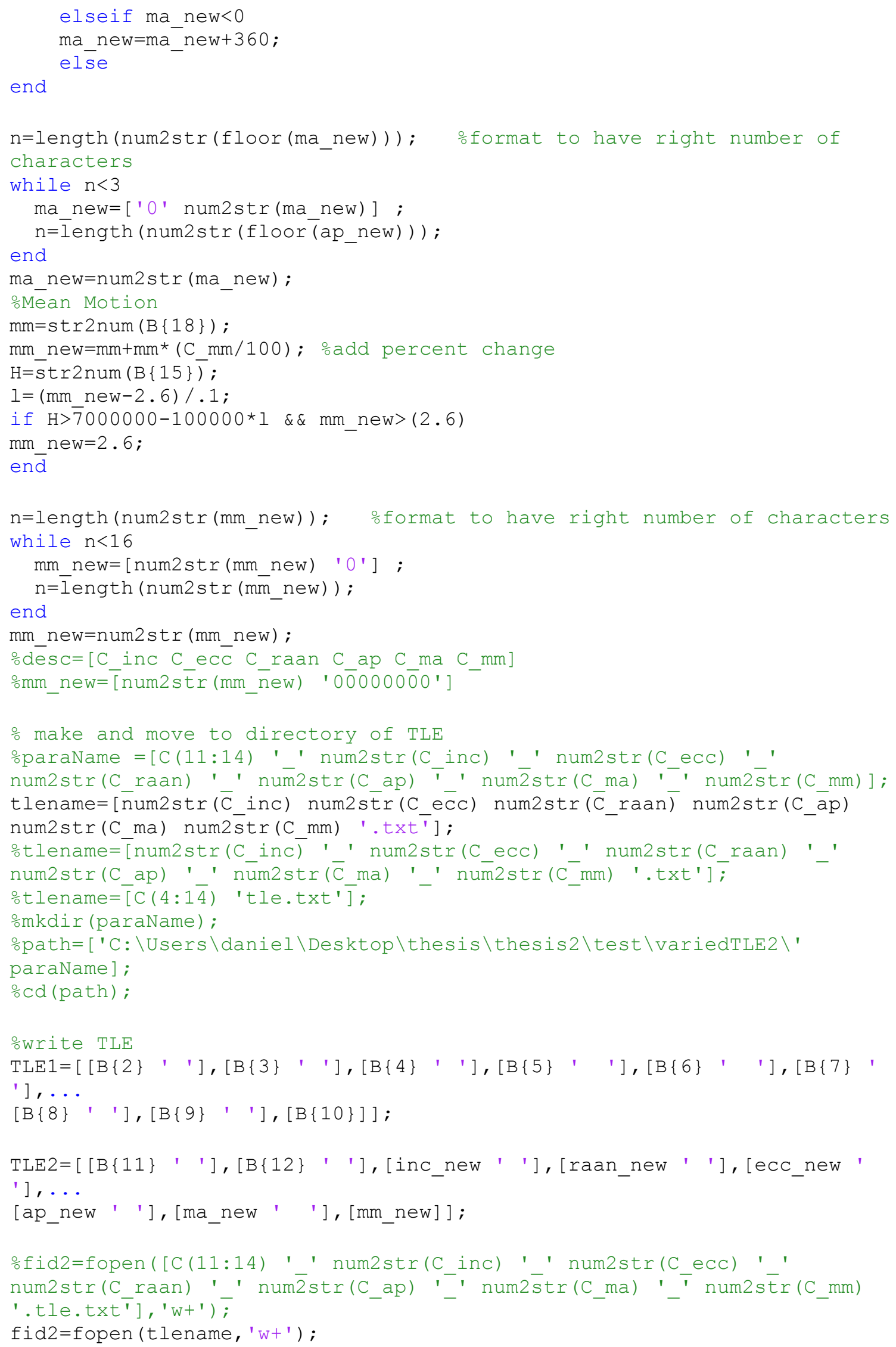




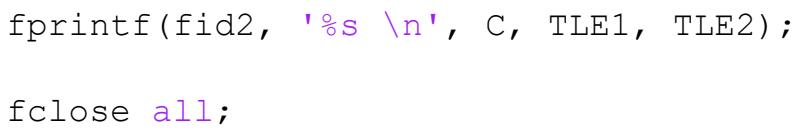




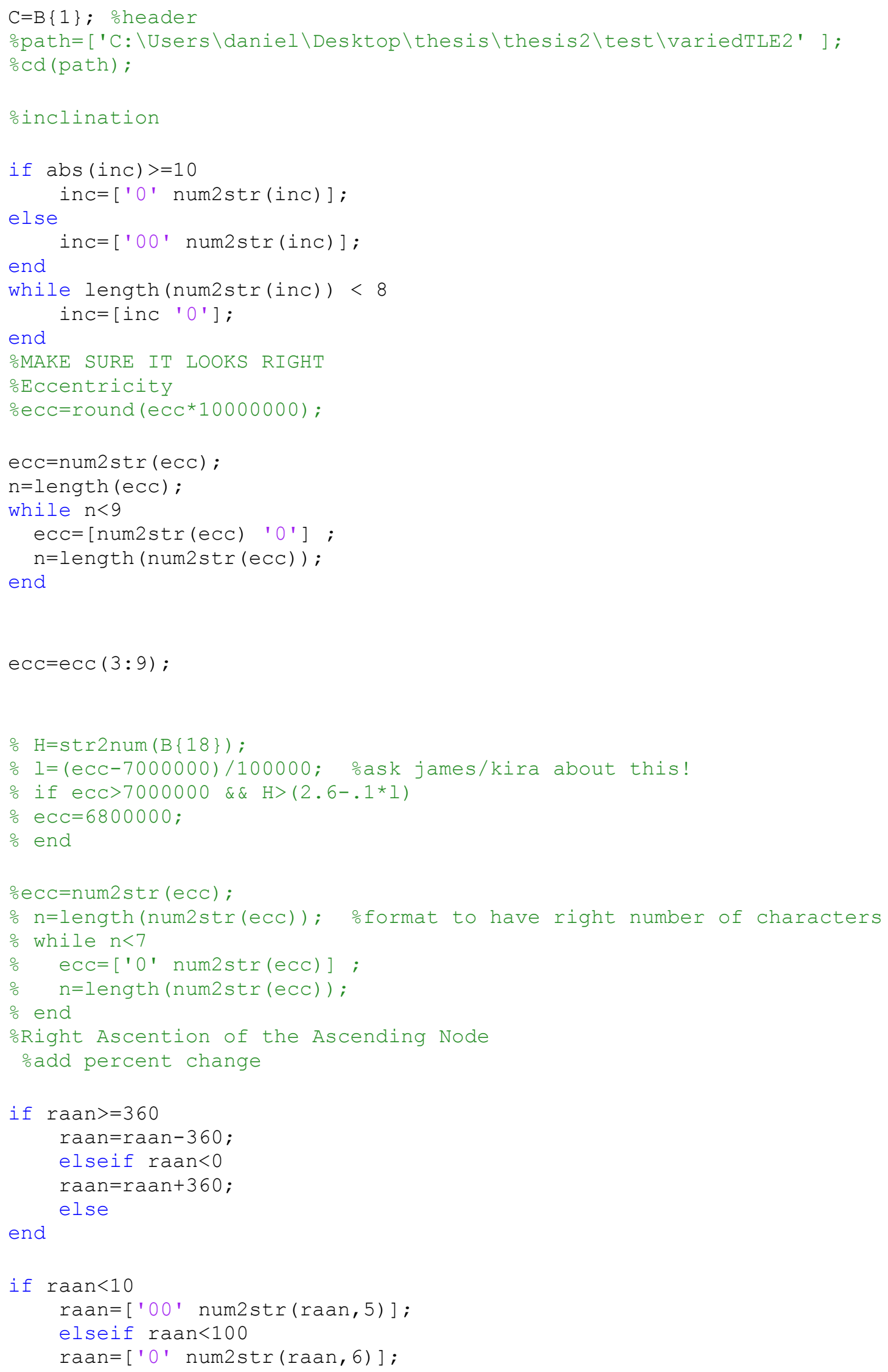




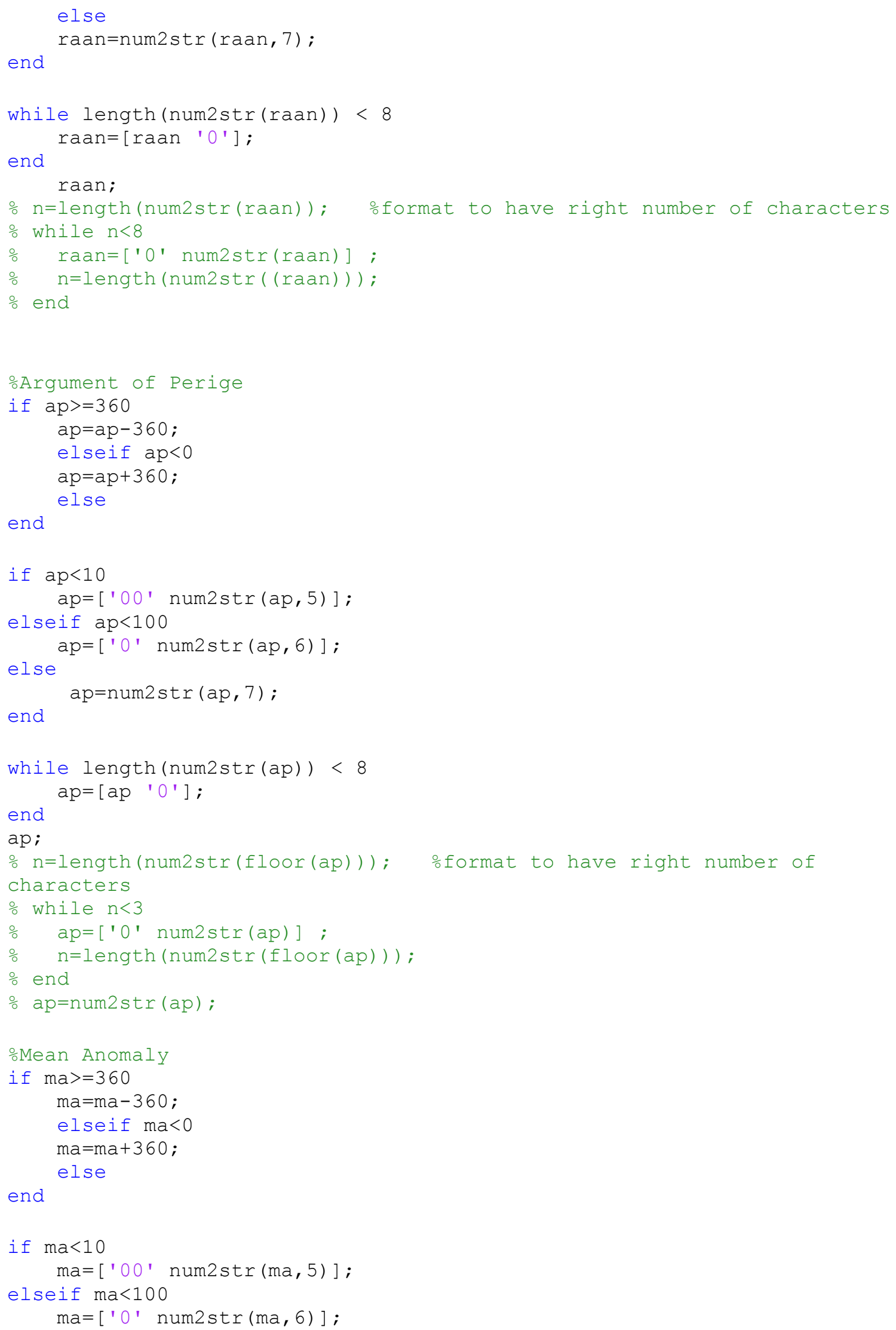




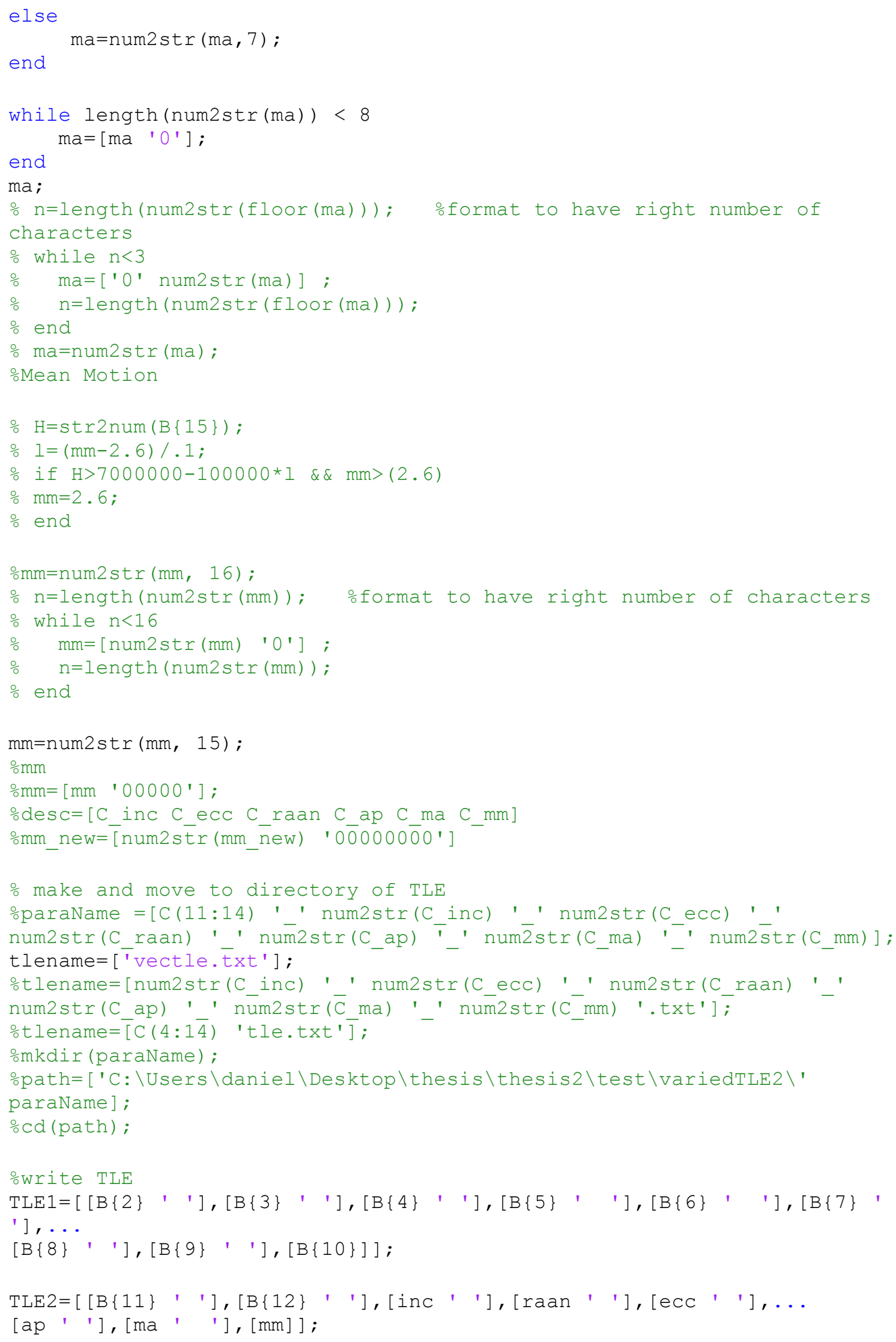




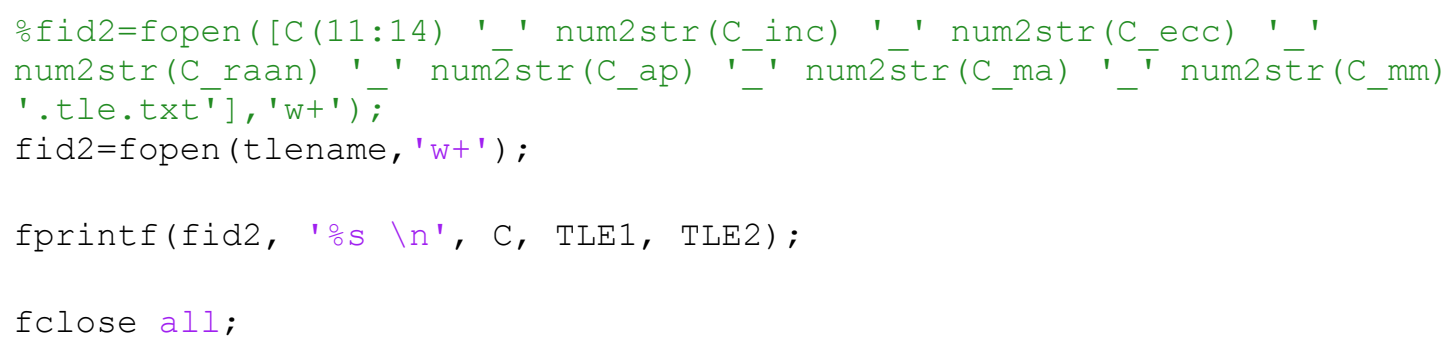




\section{Appendix B: 2007 Graphs}

\section{Object 20070750001}

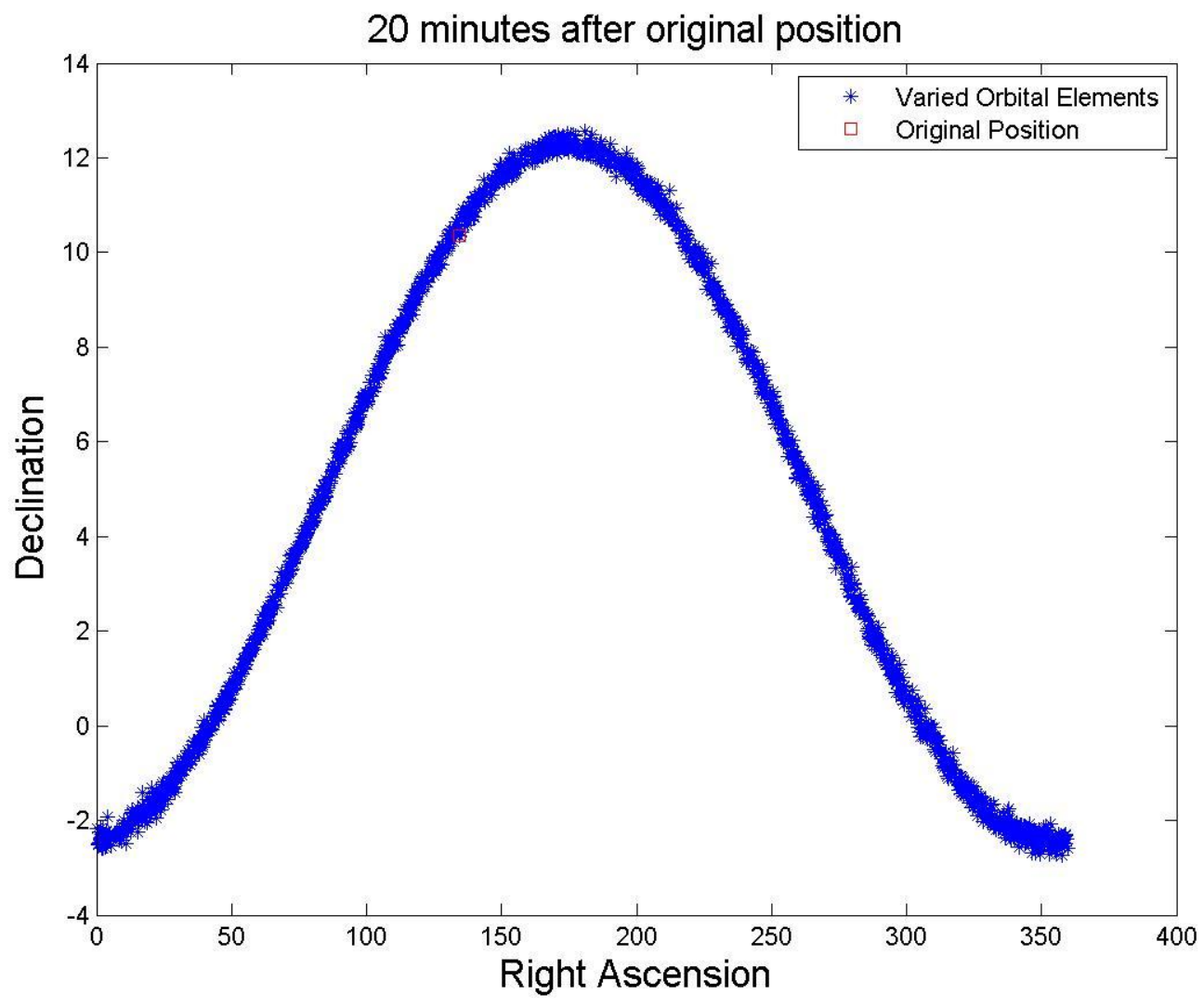



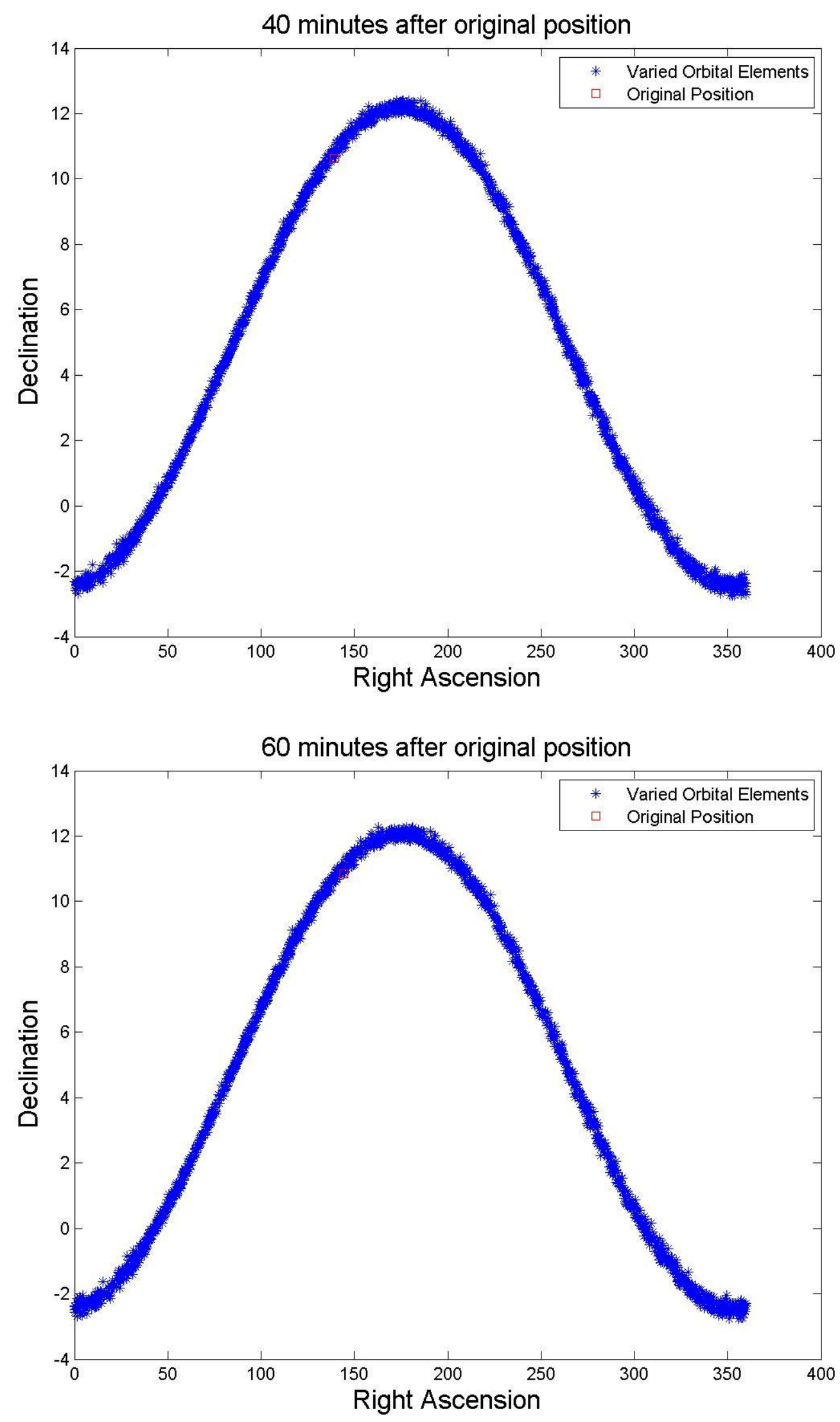


\section{Object 20070750002}

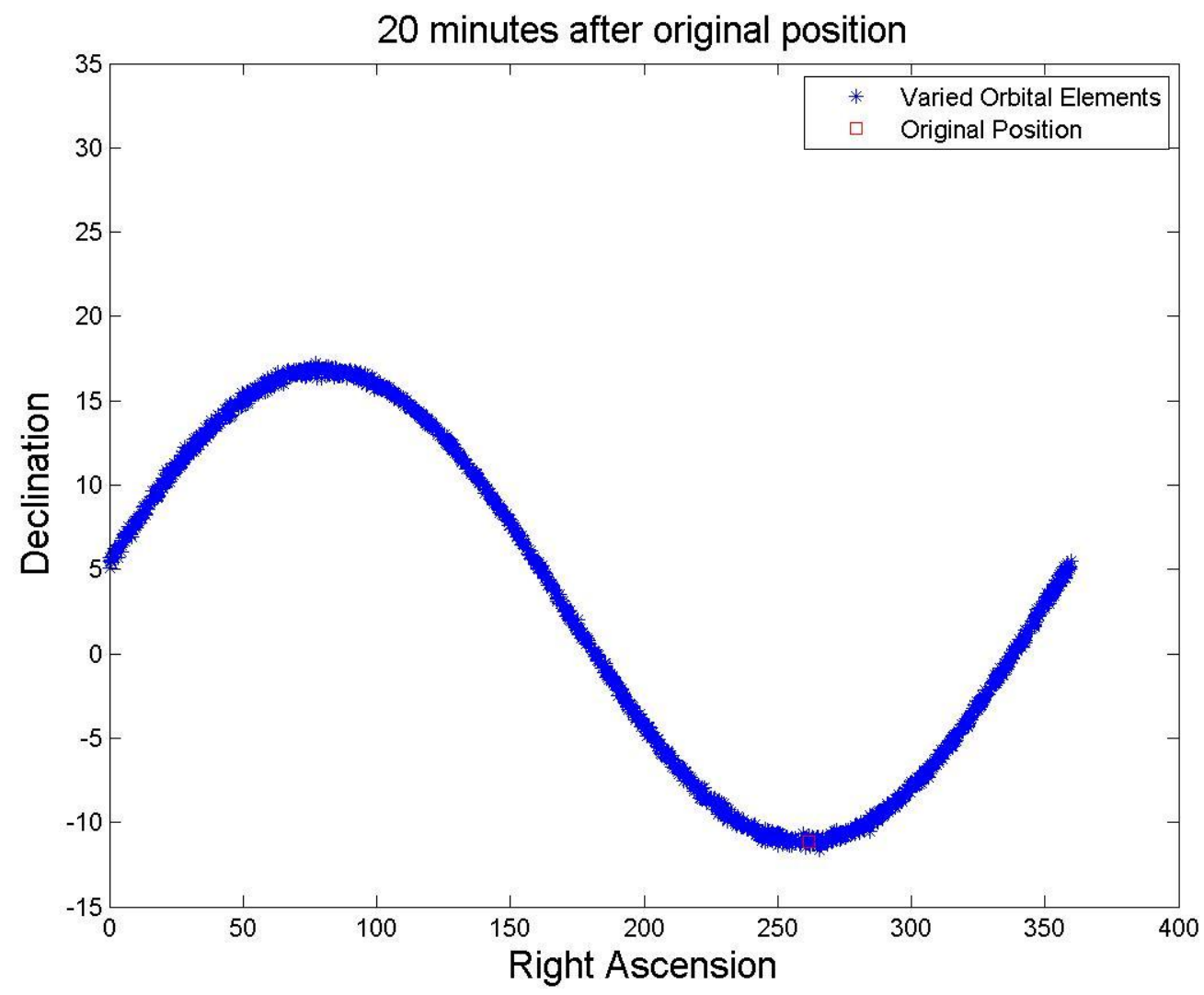



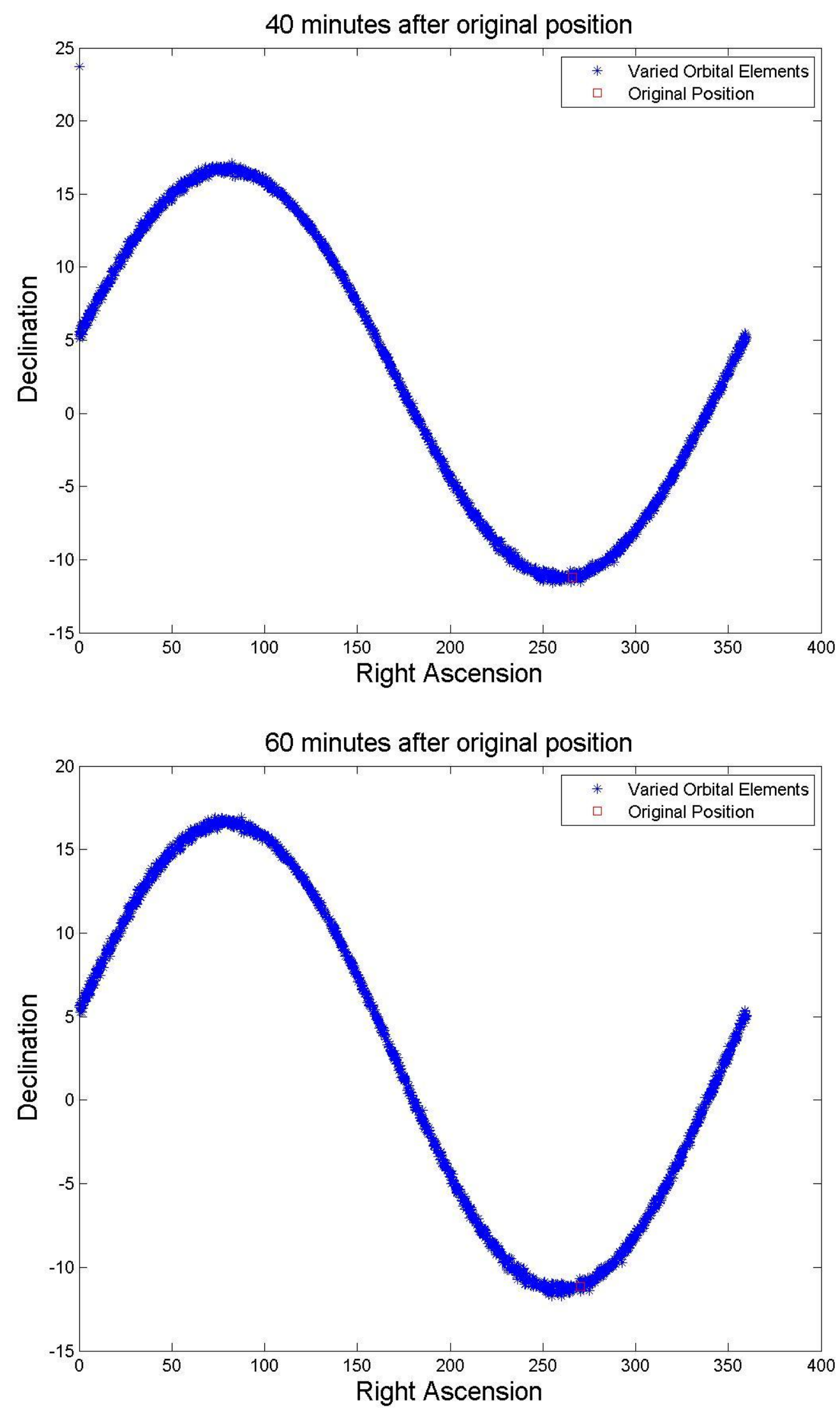


\section{Object 20070750004}

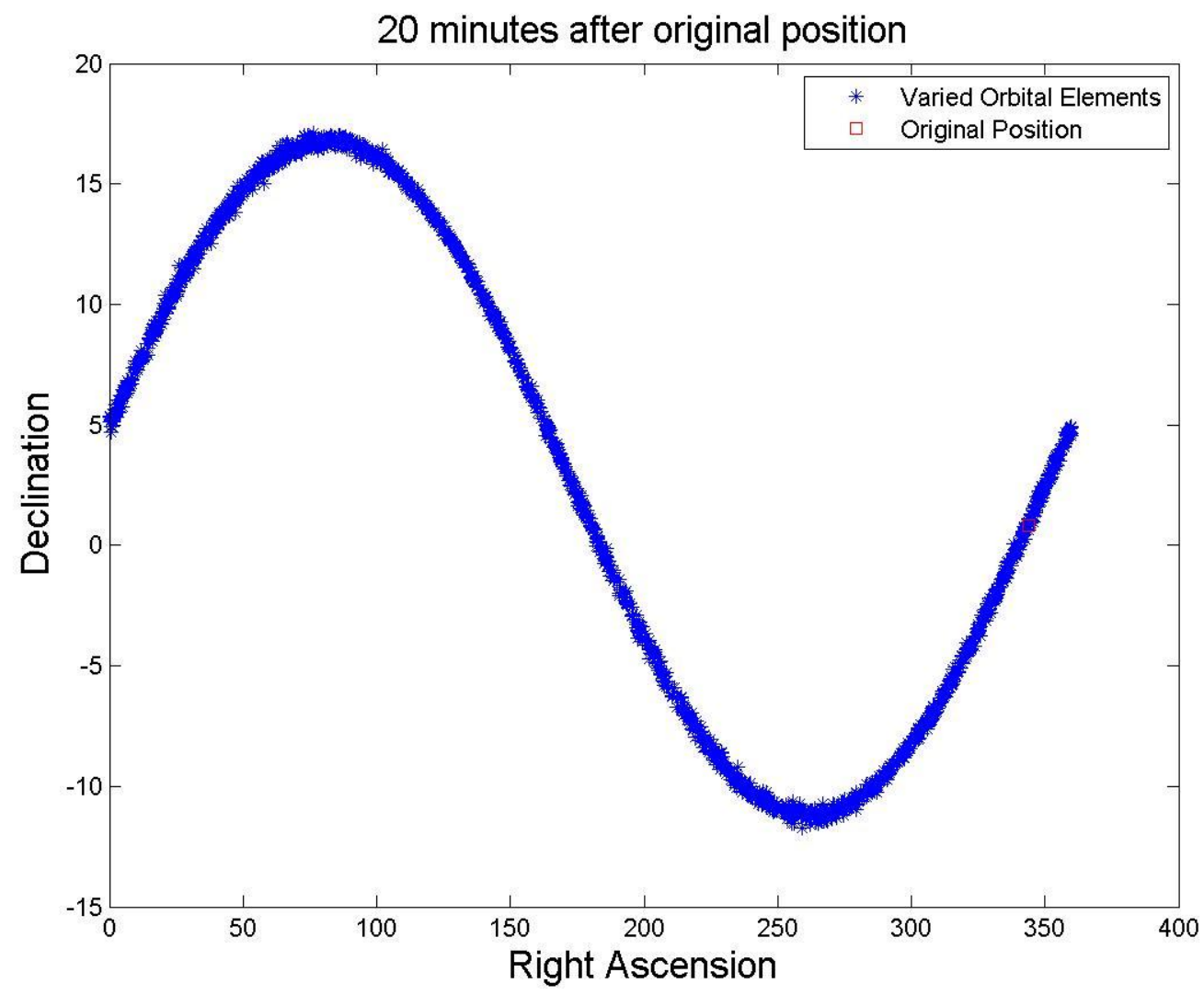



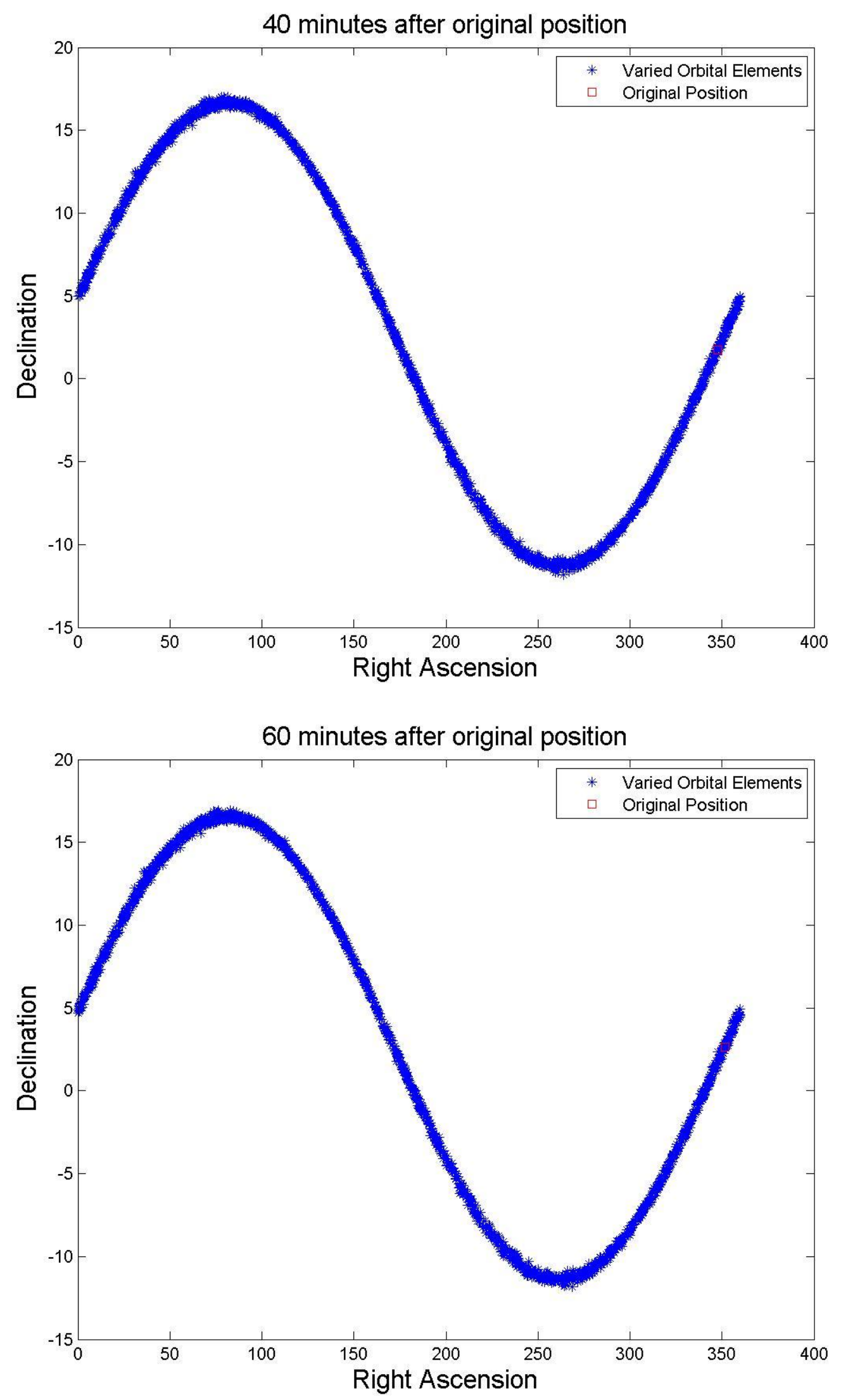


\section{Object 20070760001}

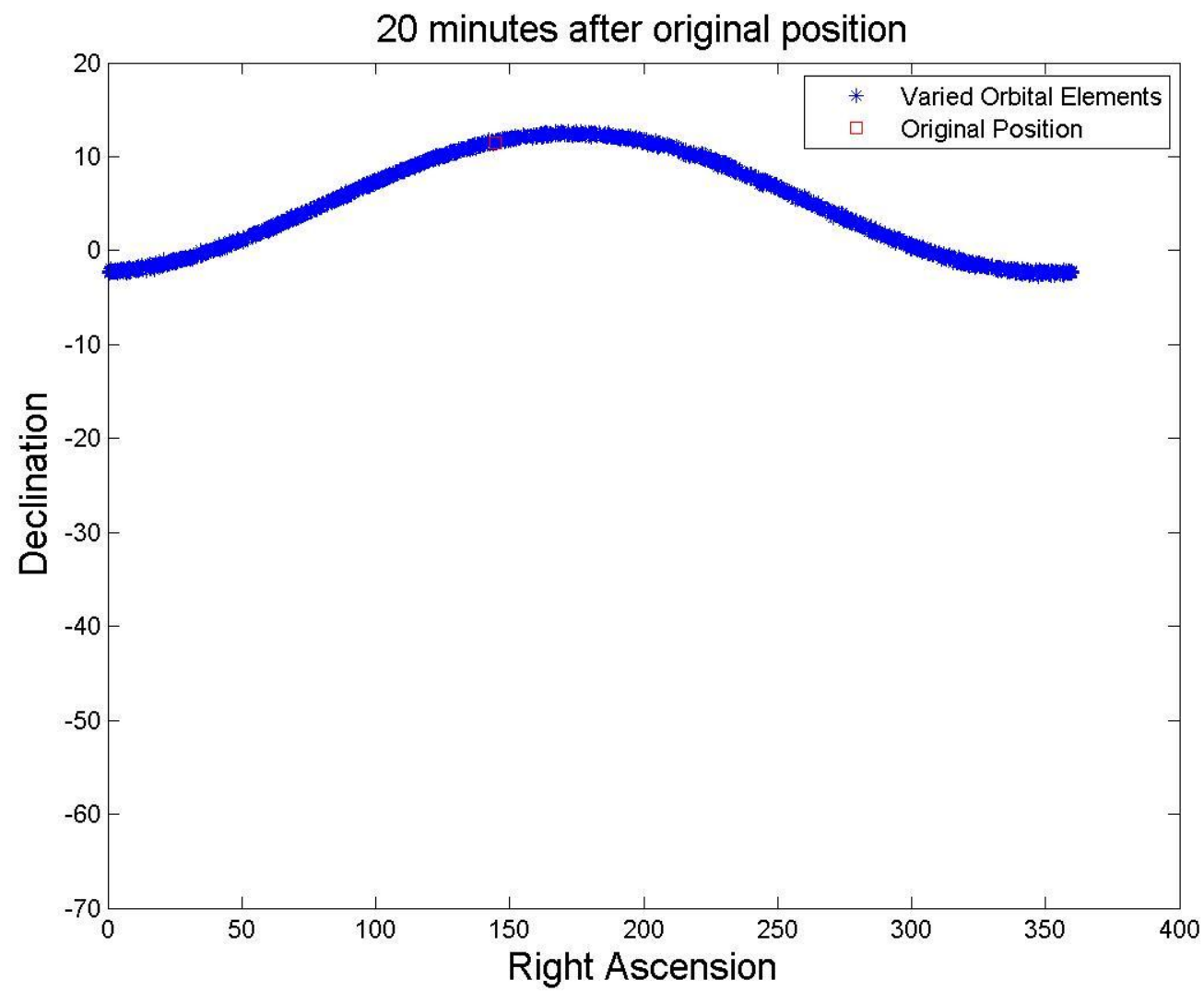



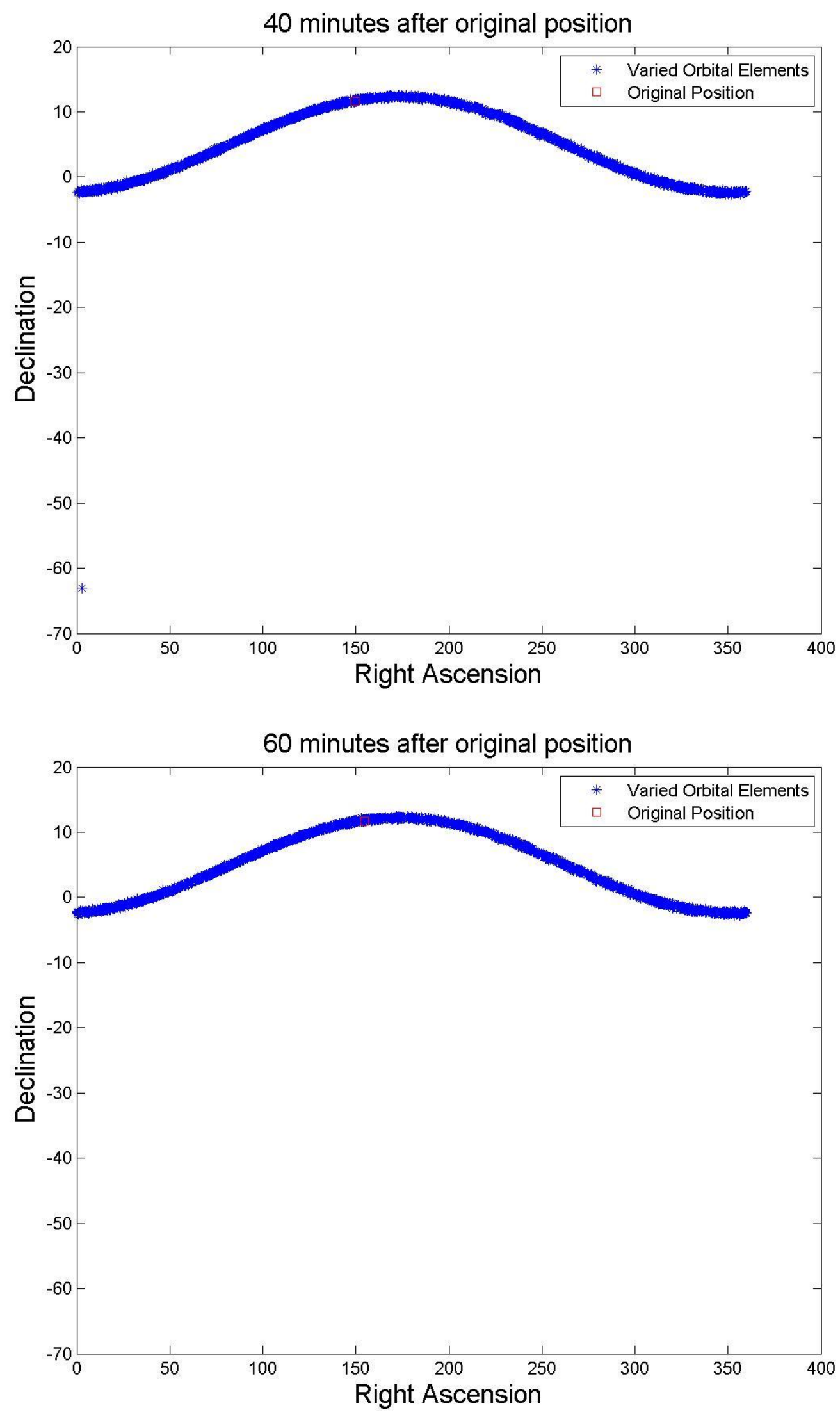


\section{Object 20070760002}

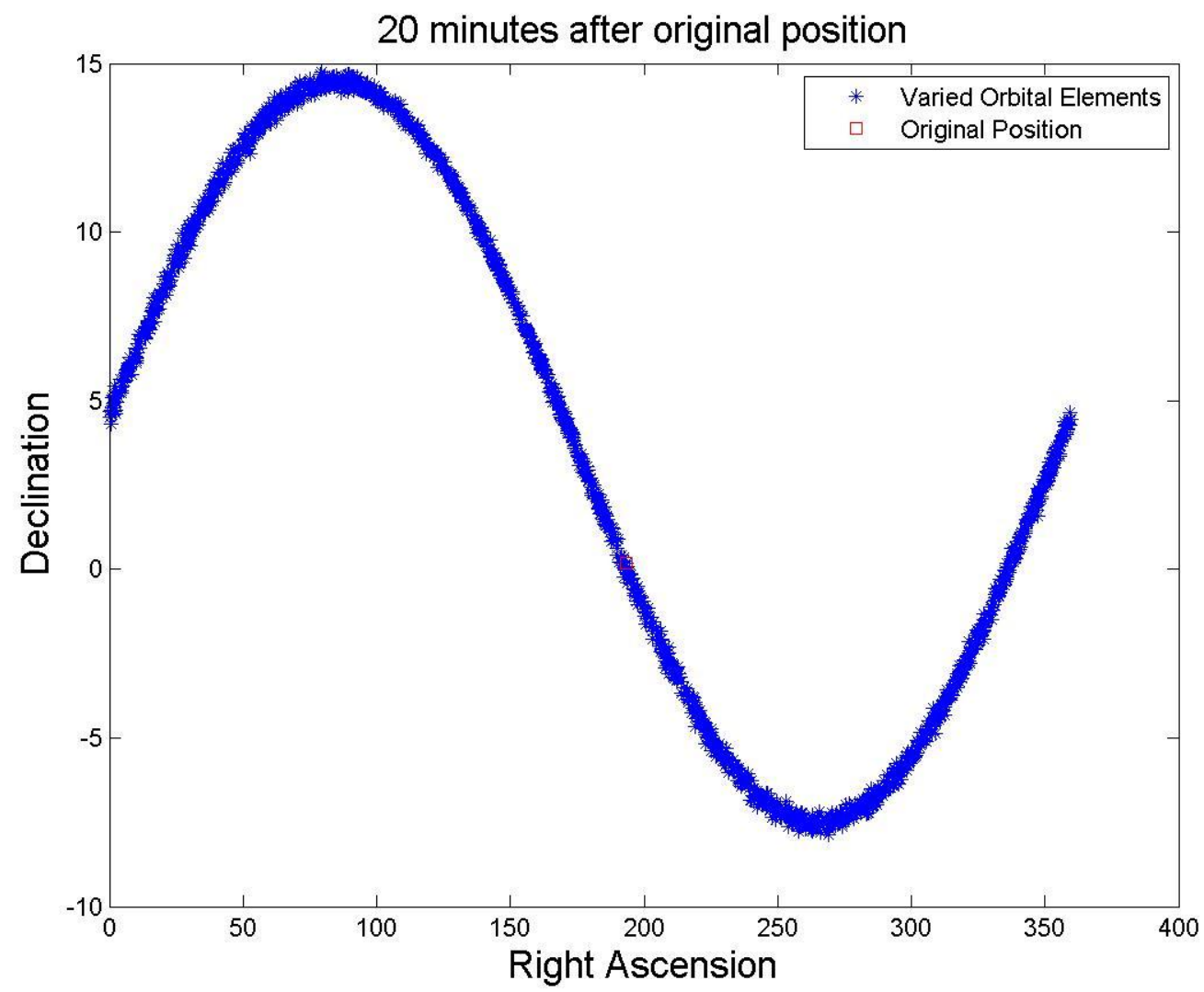



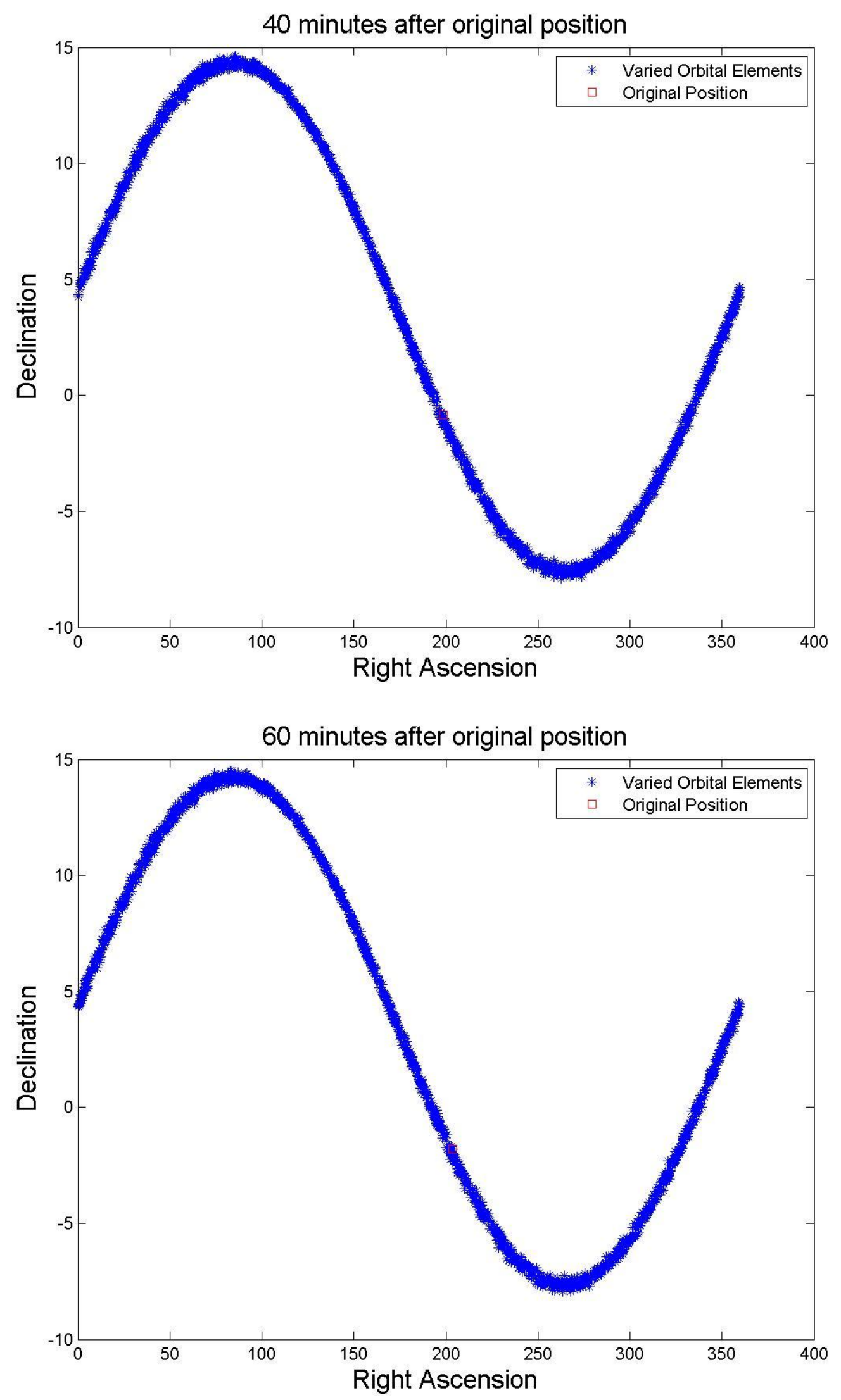


\section{Object 20070760003}

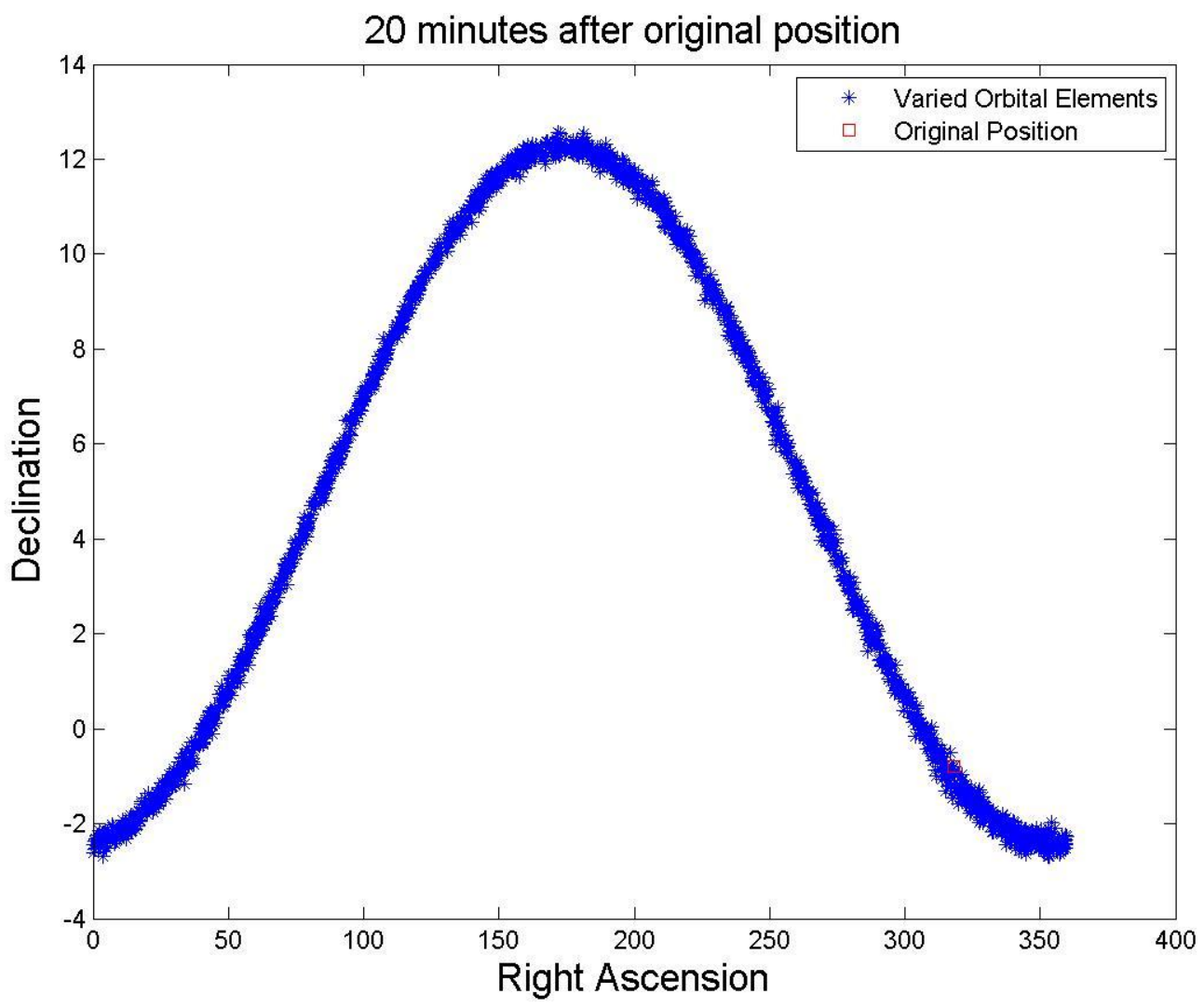



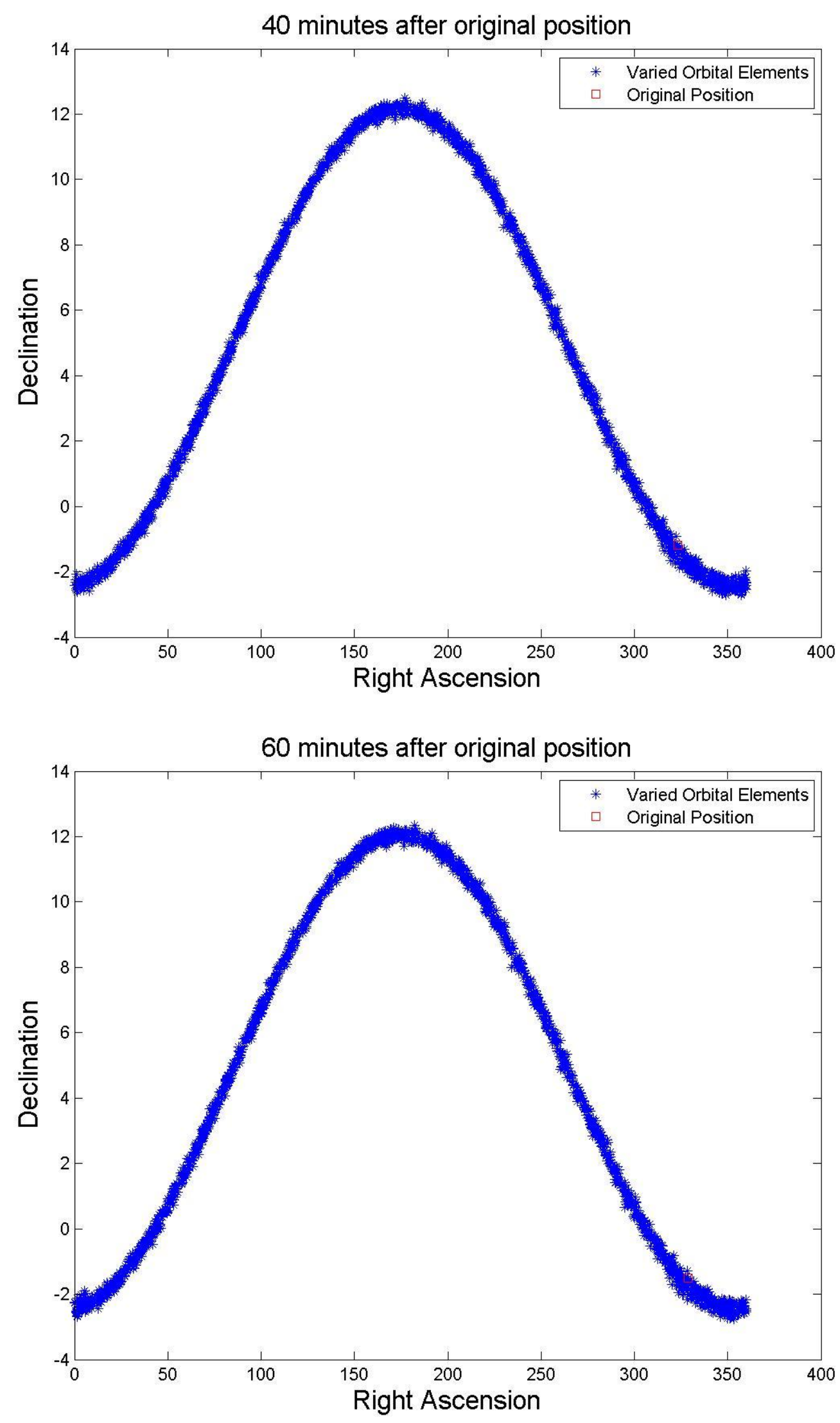


\section{Object 20070760005}

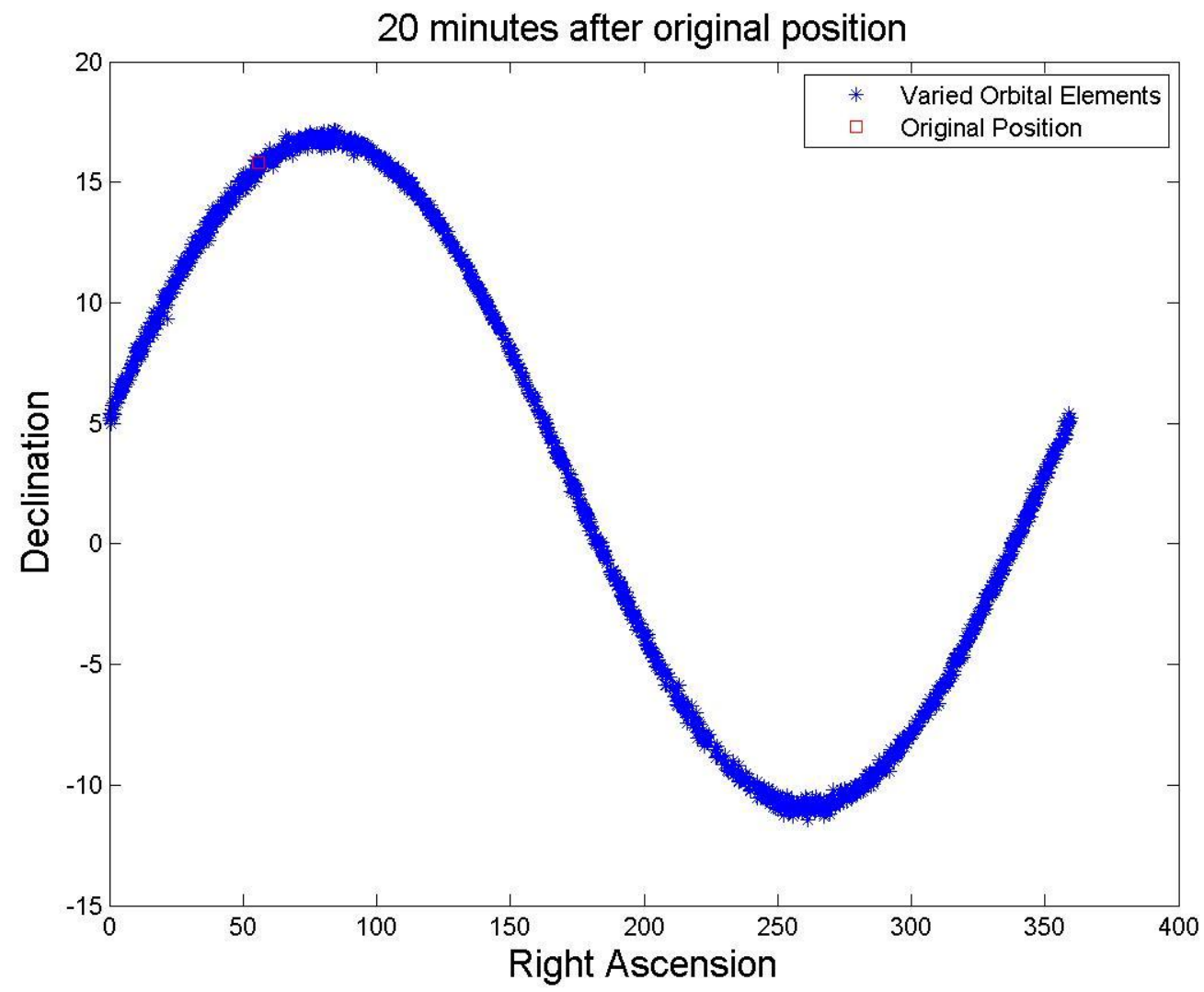



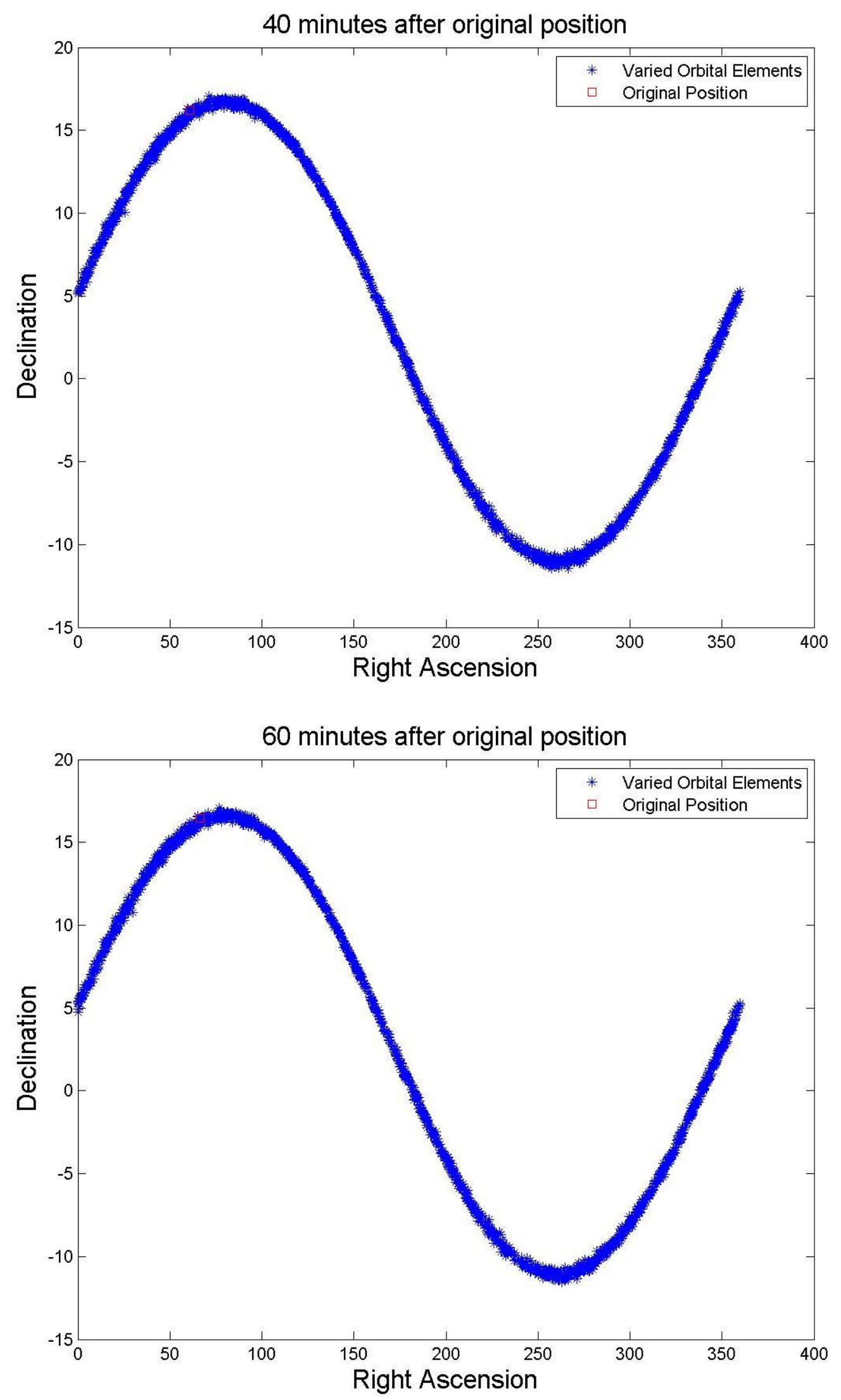


\section{Object 20070760009}

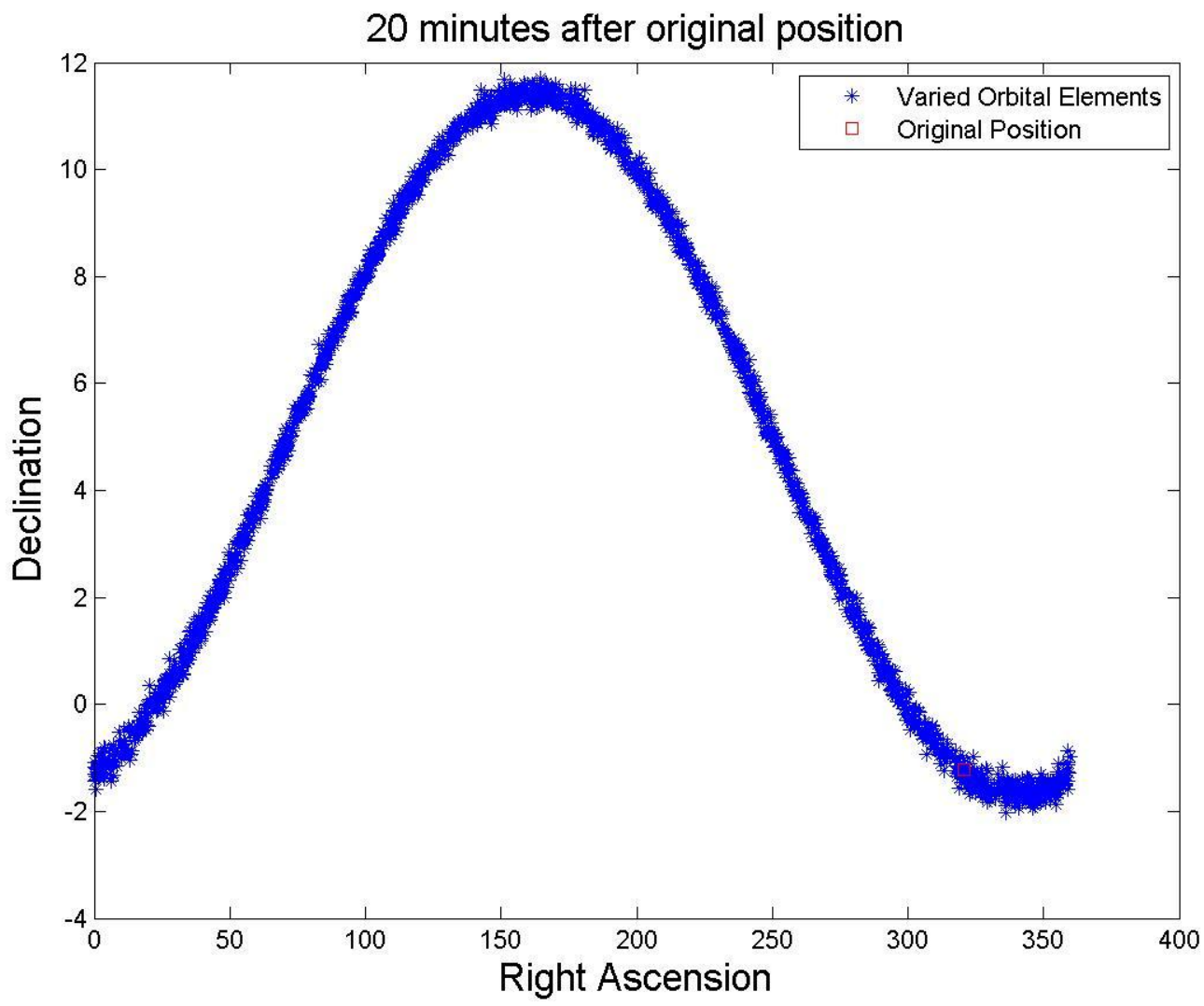



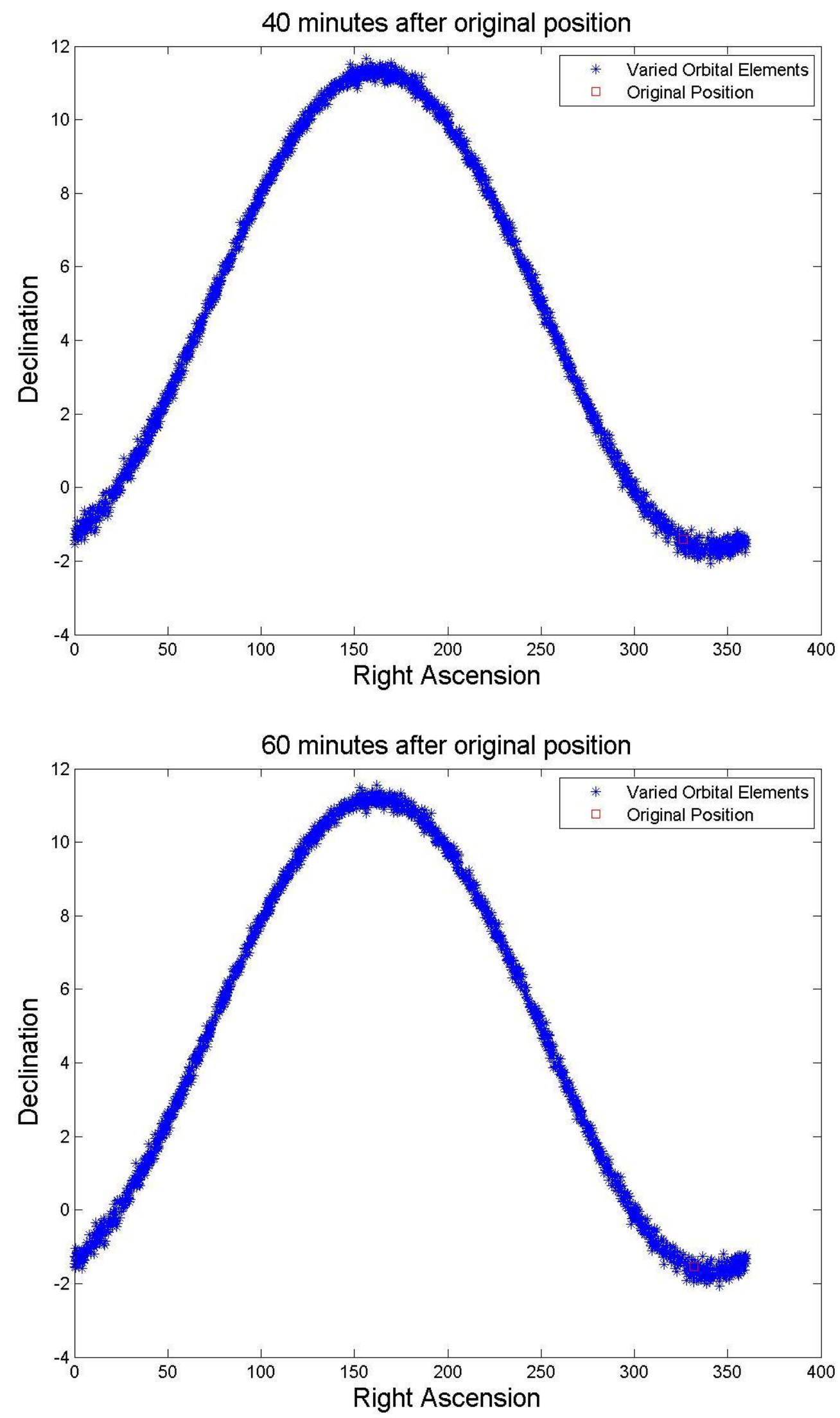


\section{Object 20070770001}

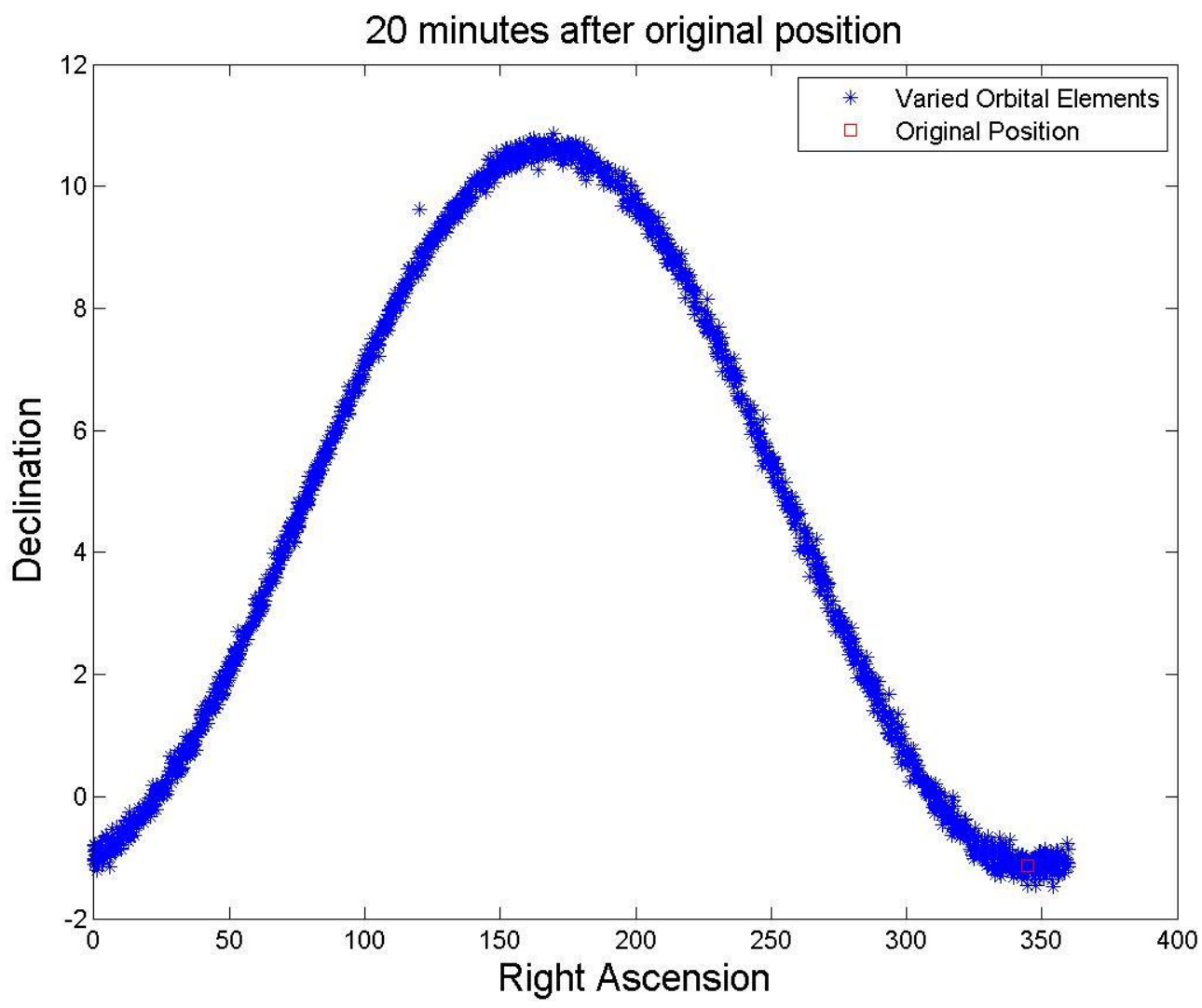



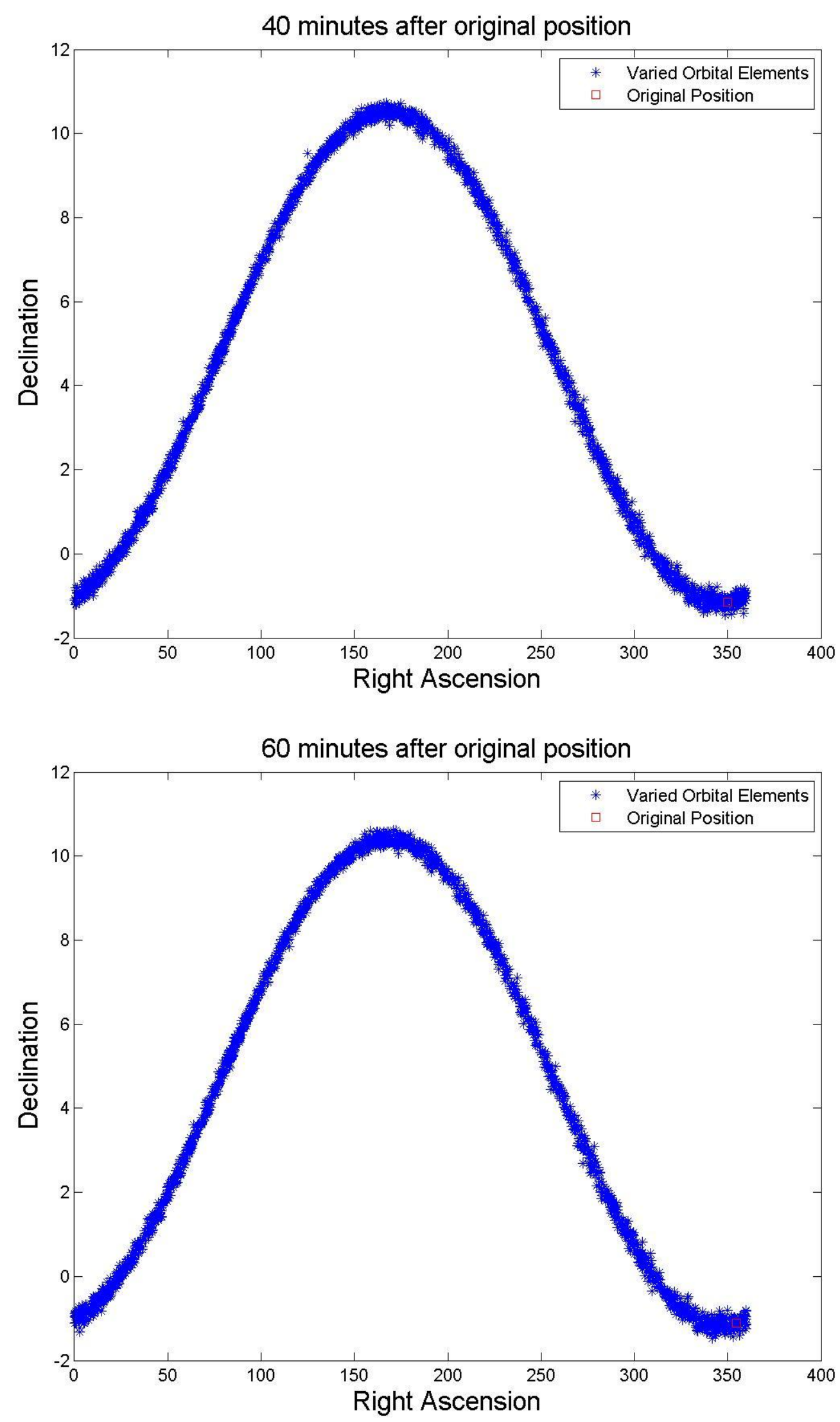


\section{Object 20070770002}

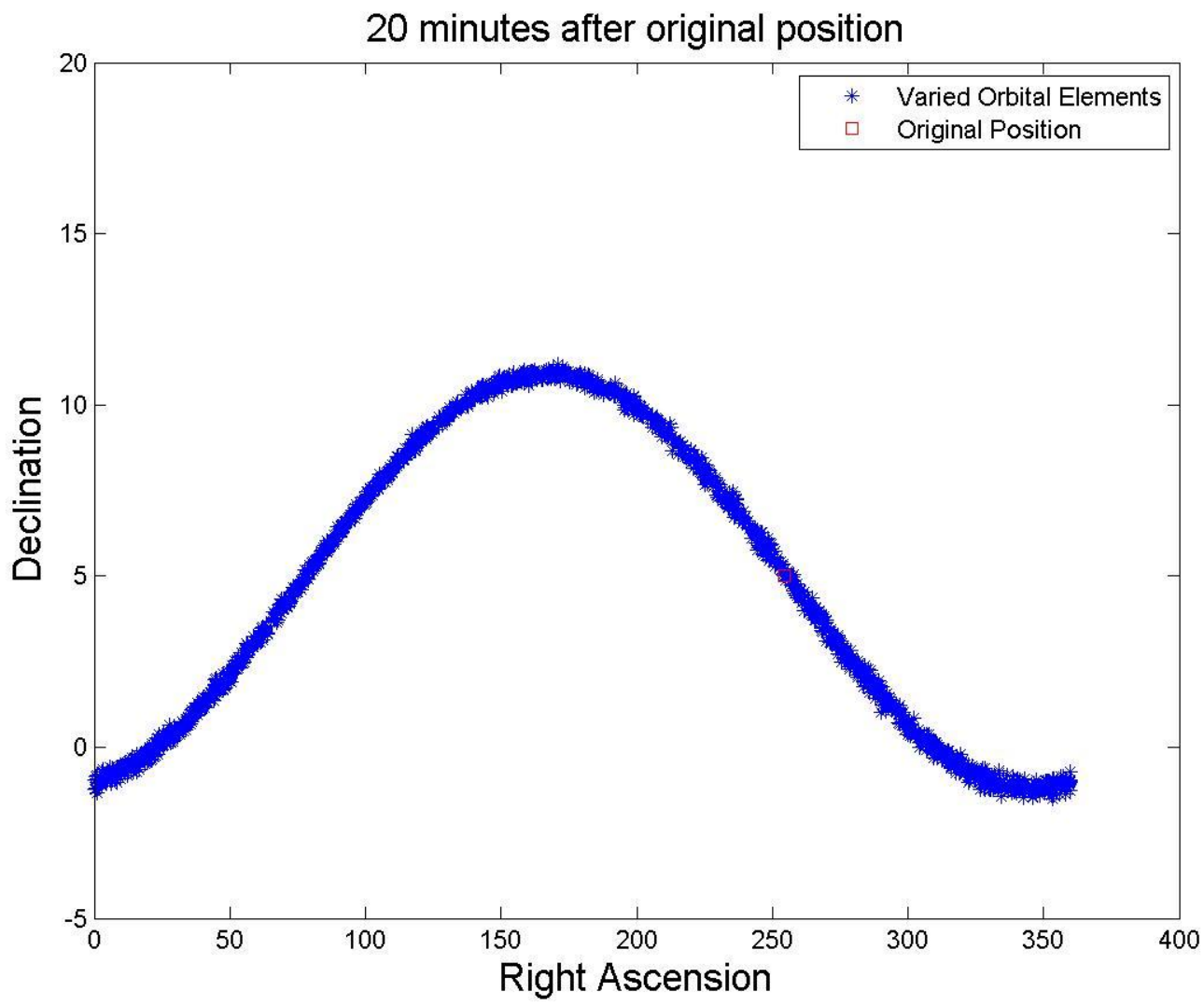



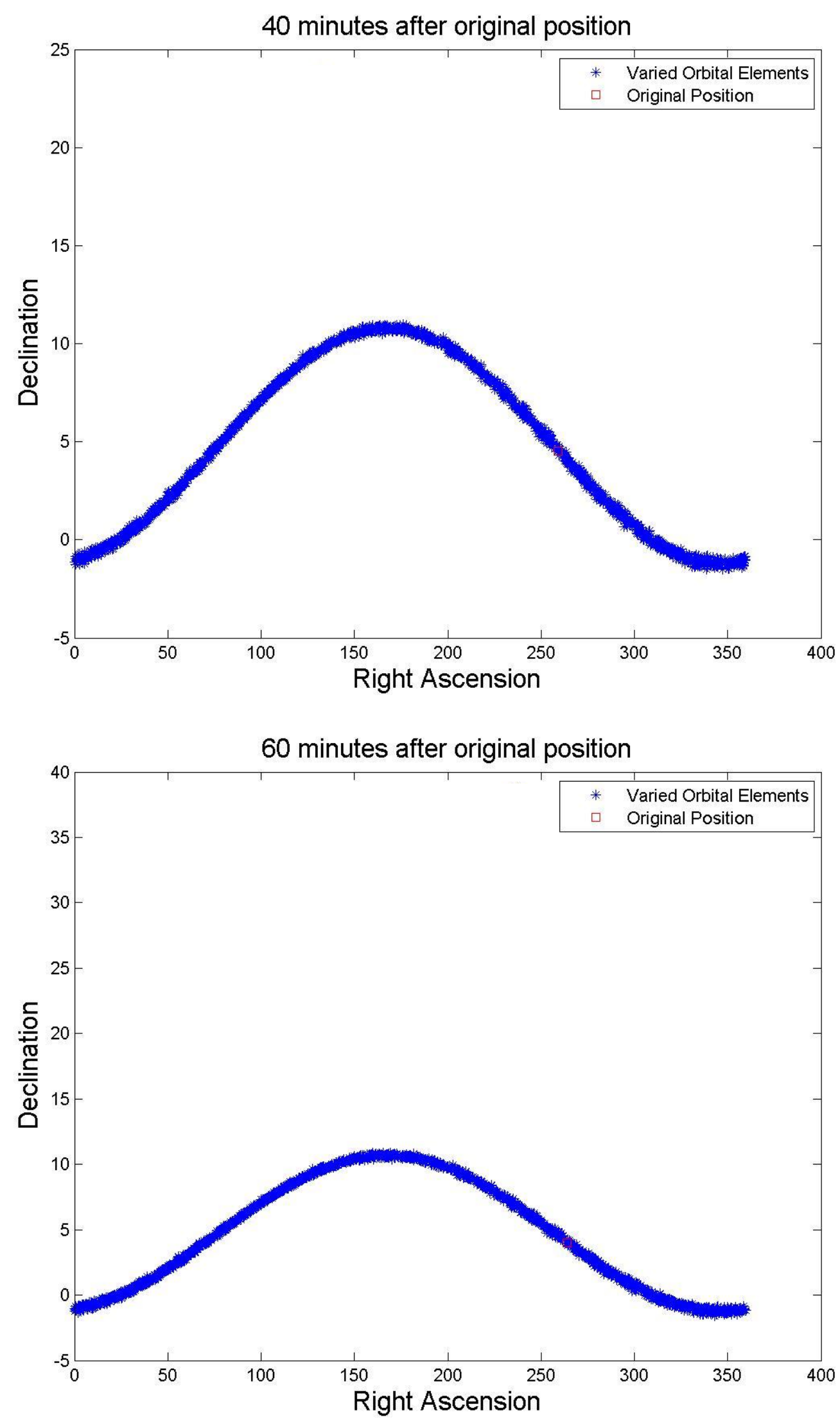


\section{Object 20070770003}

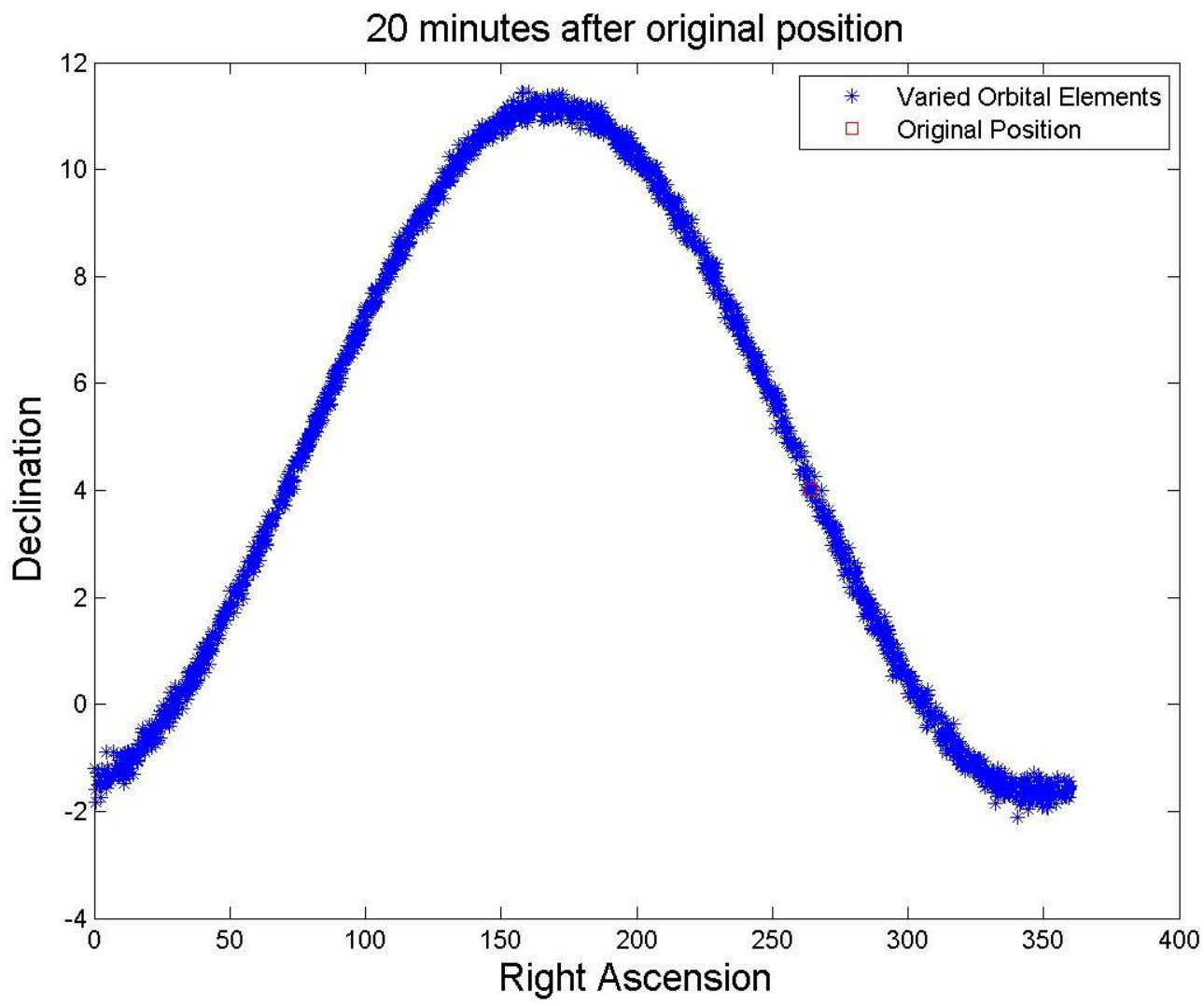



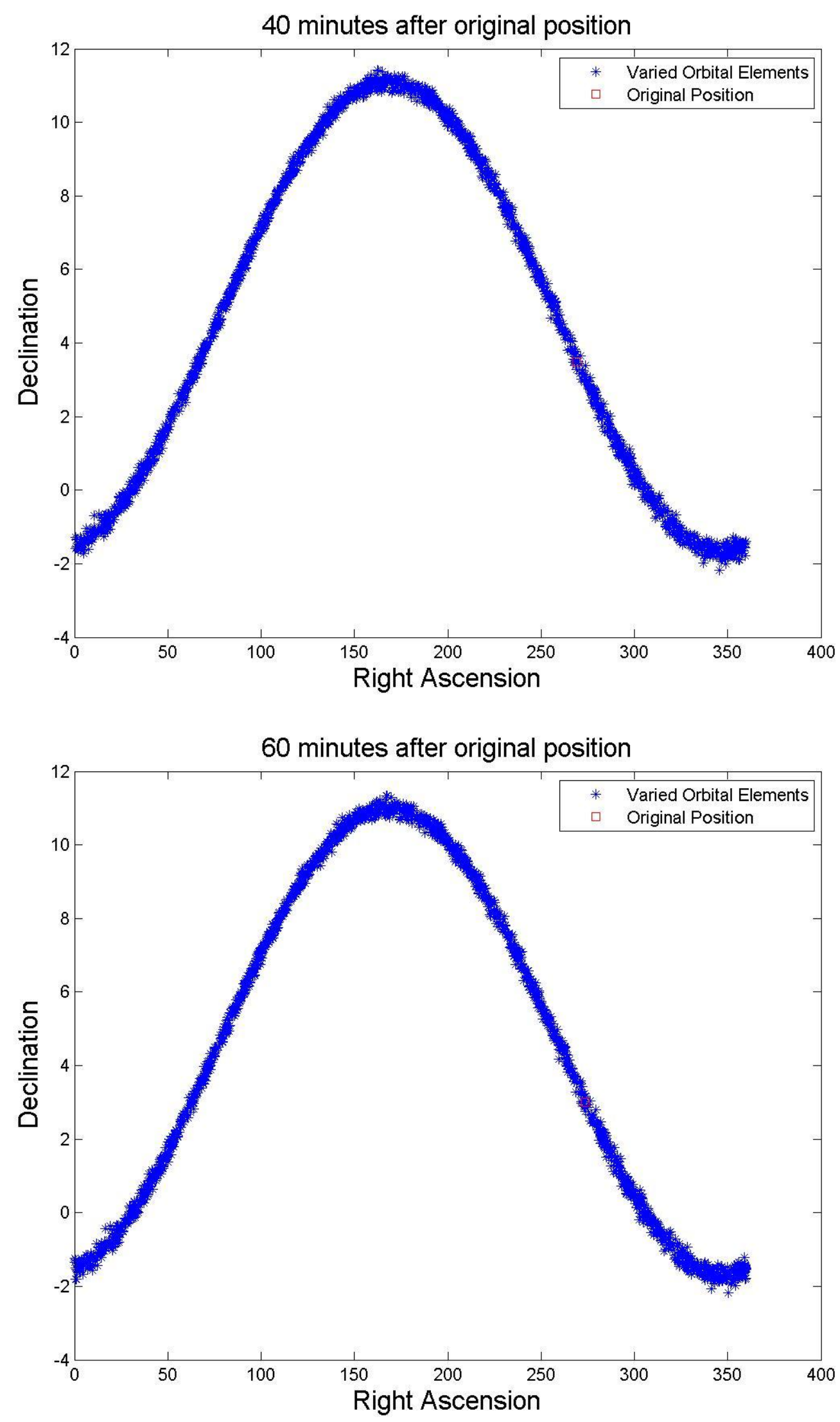


\section{Object 20070770007}

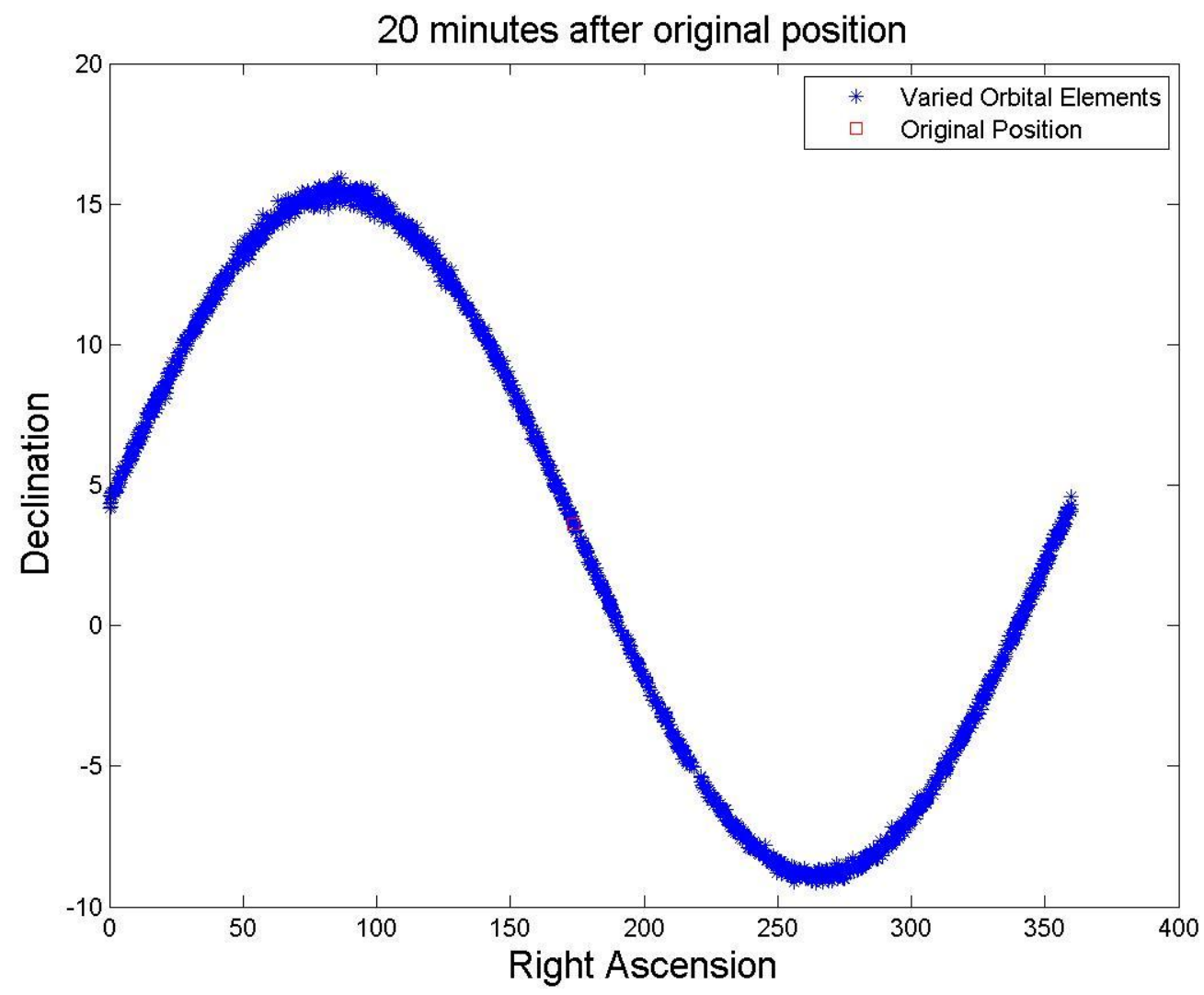



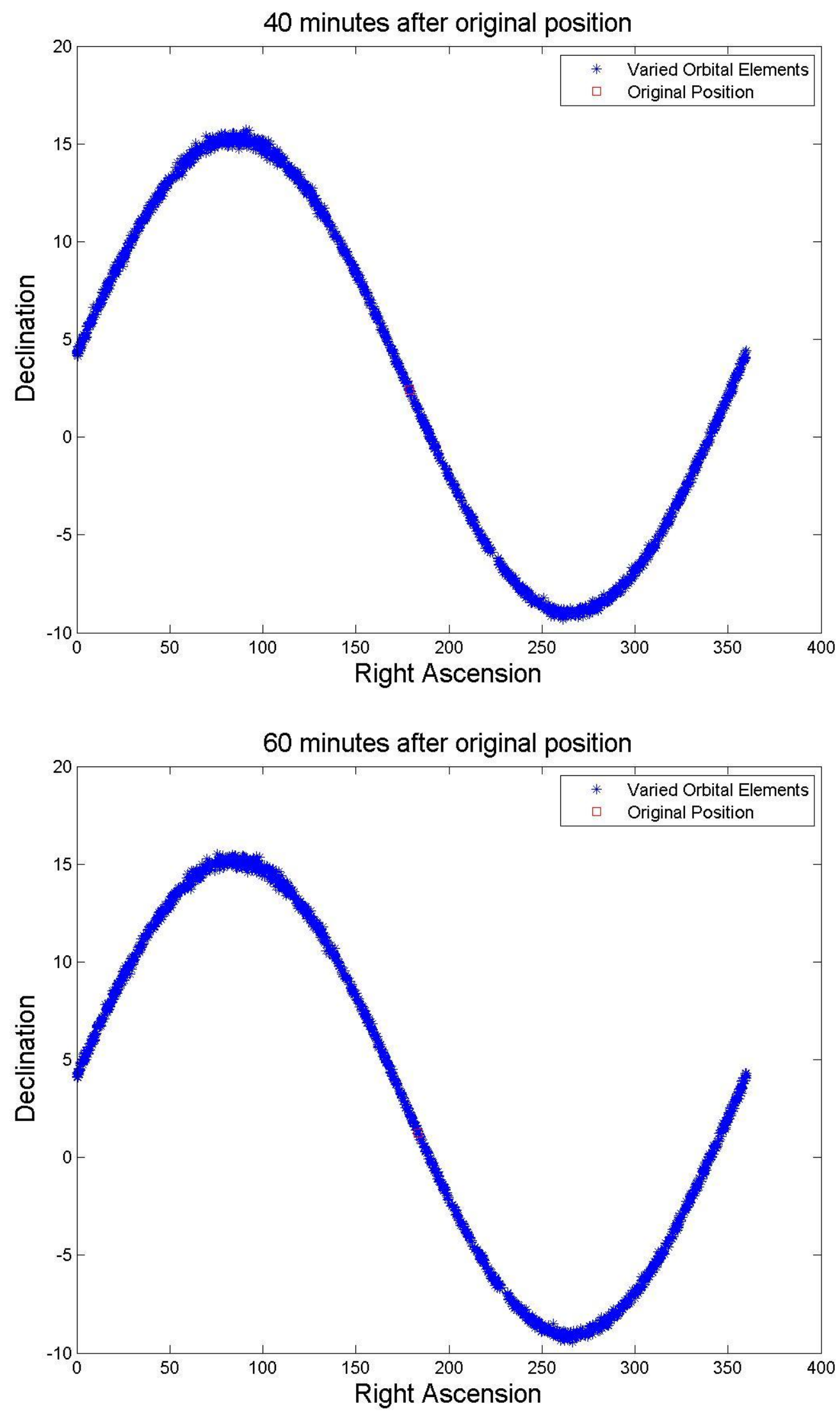


\section{Object 20070771620}

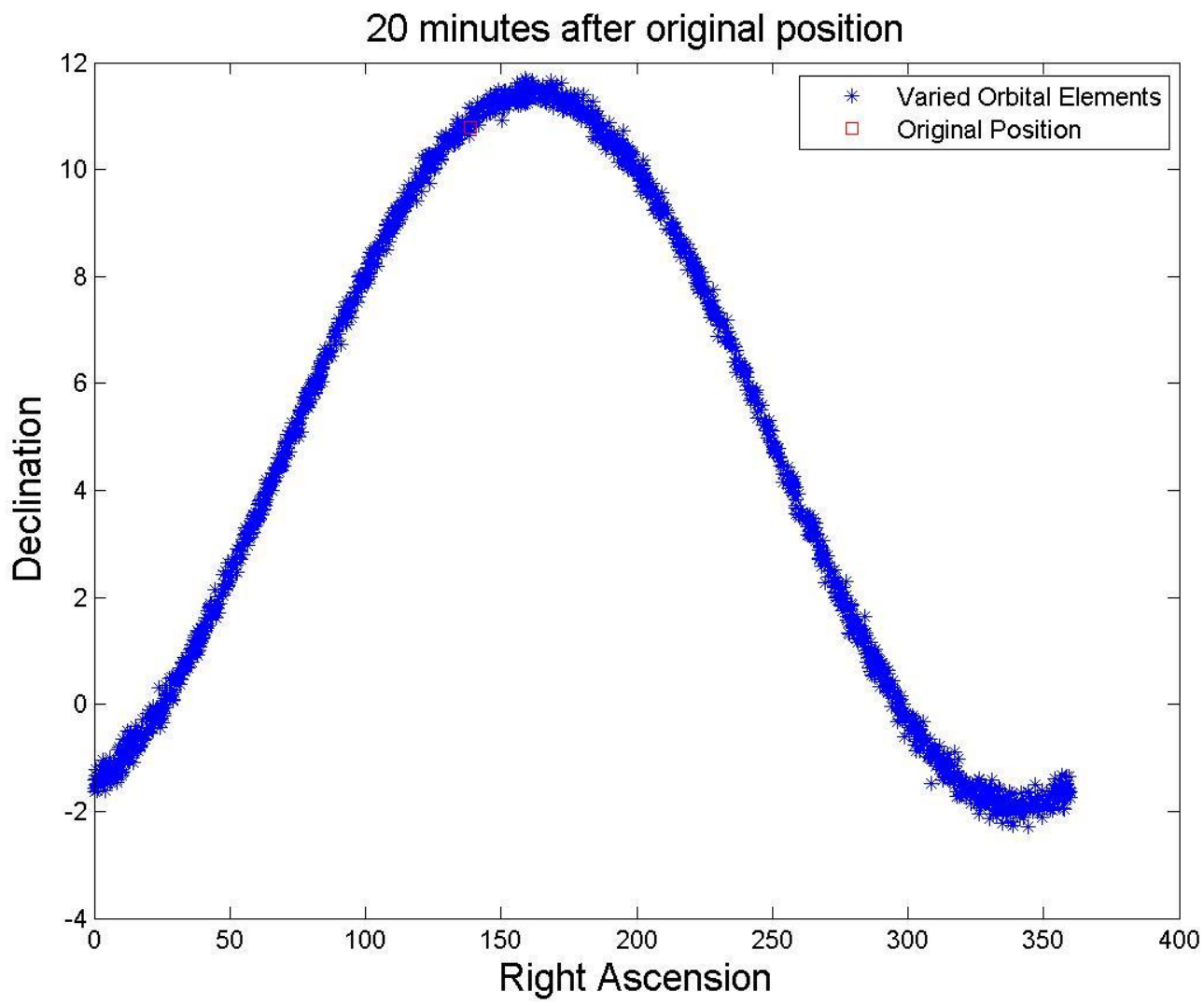



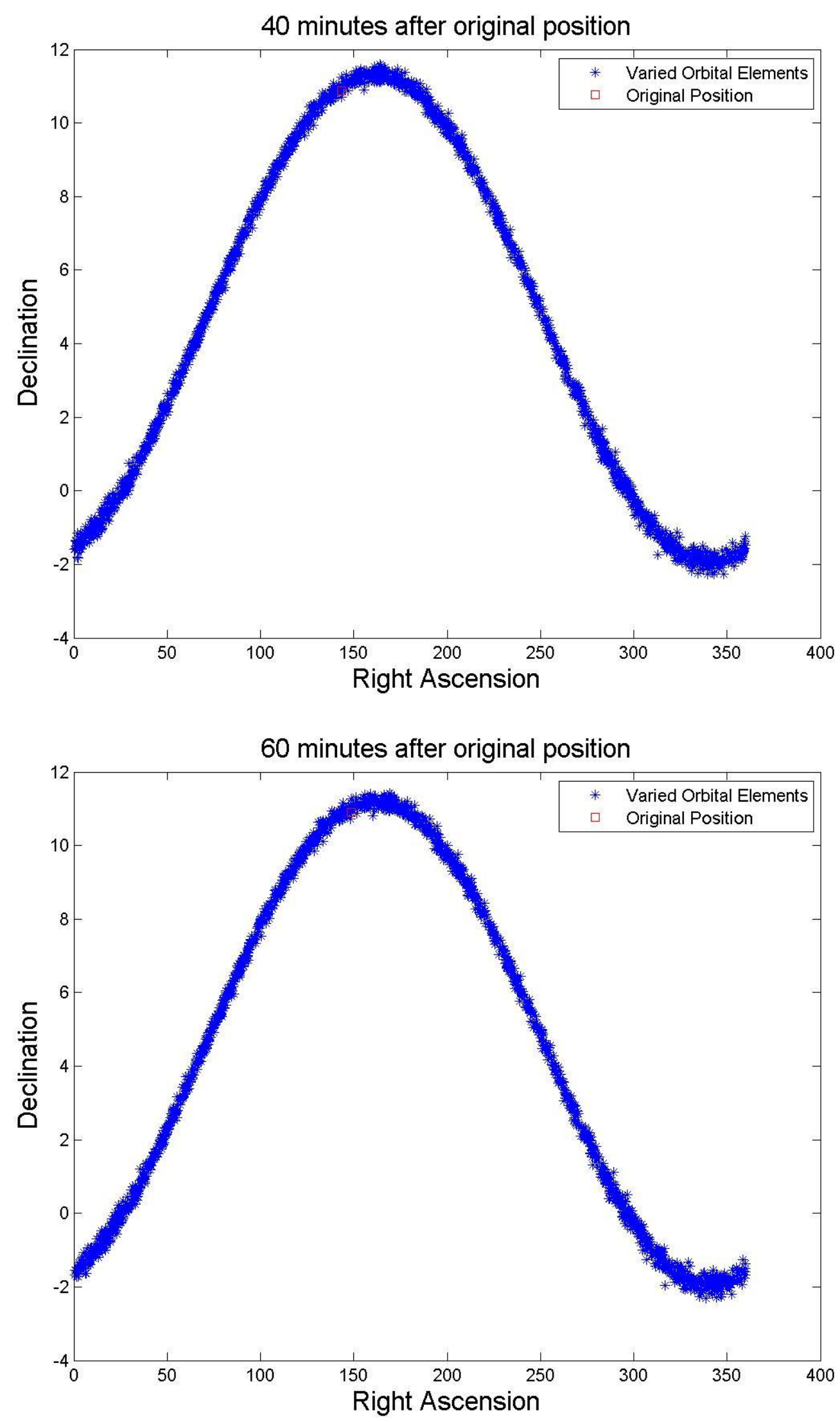


\section{Object 20070771921}

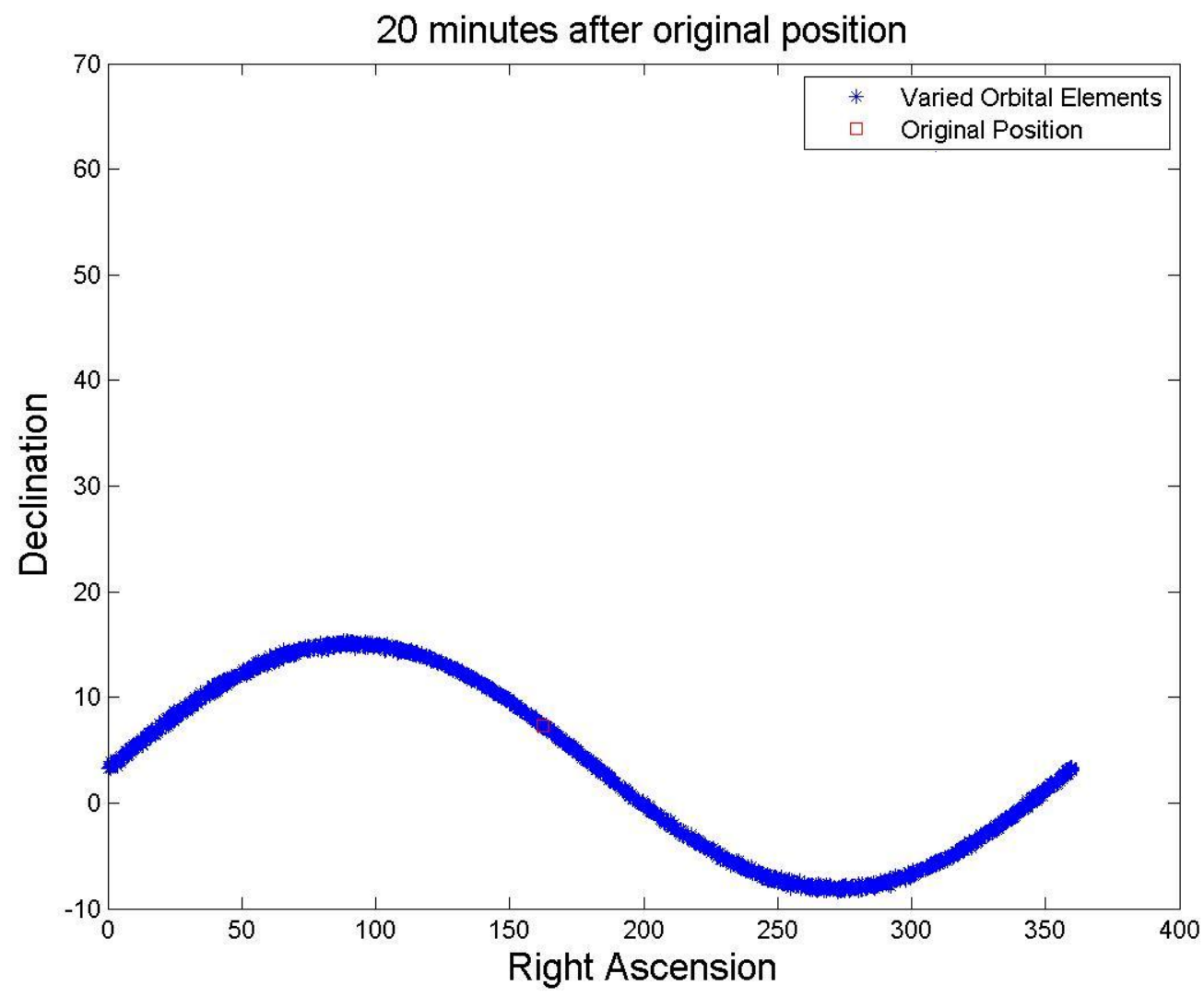



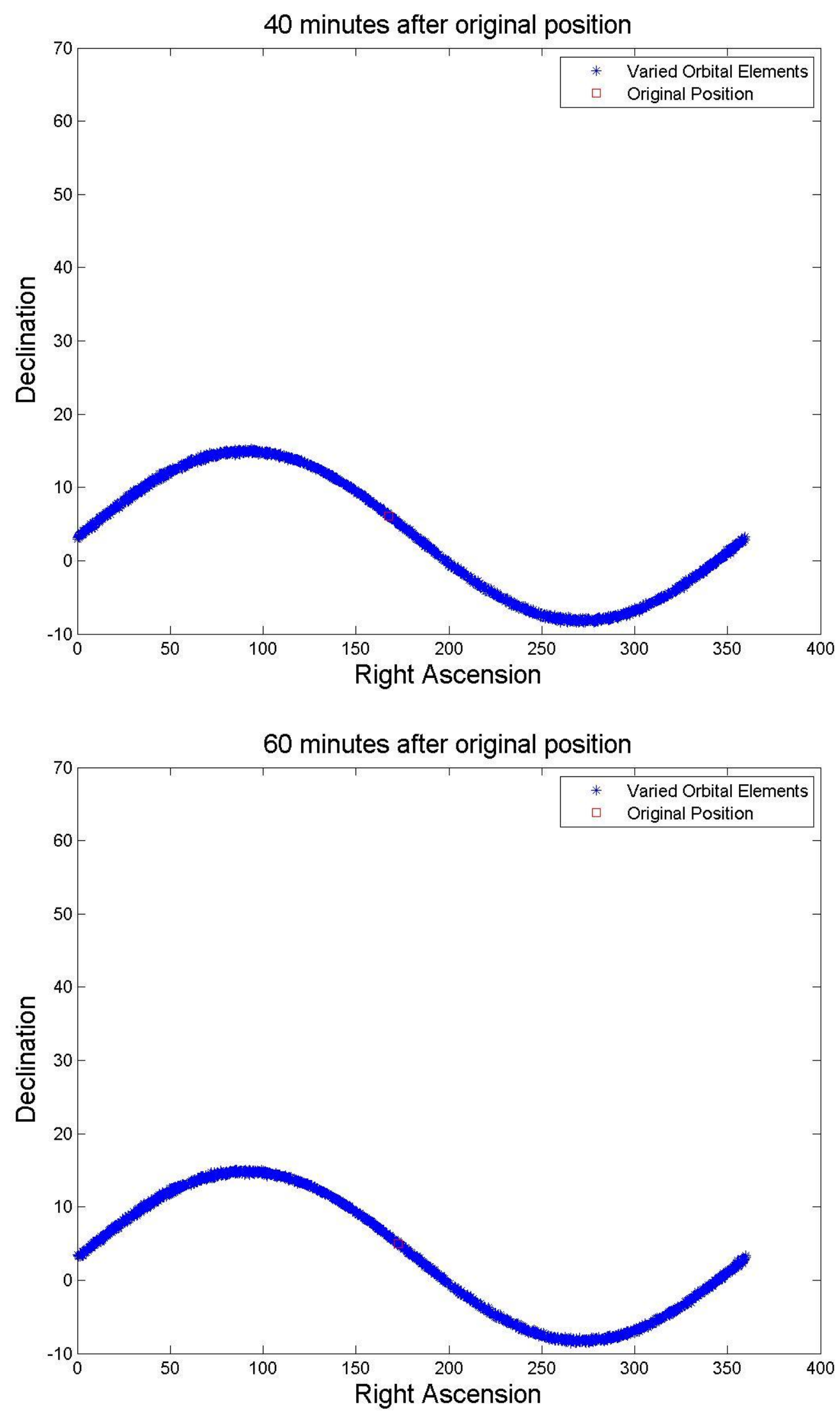


\section{Object 20070772128}

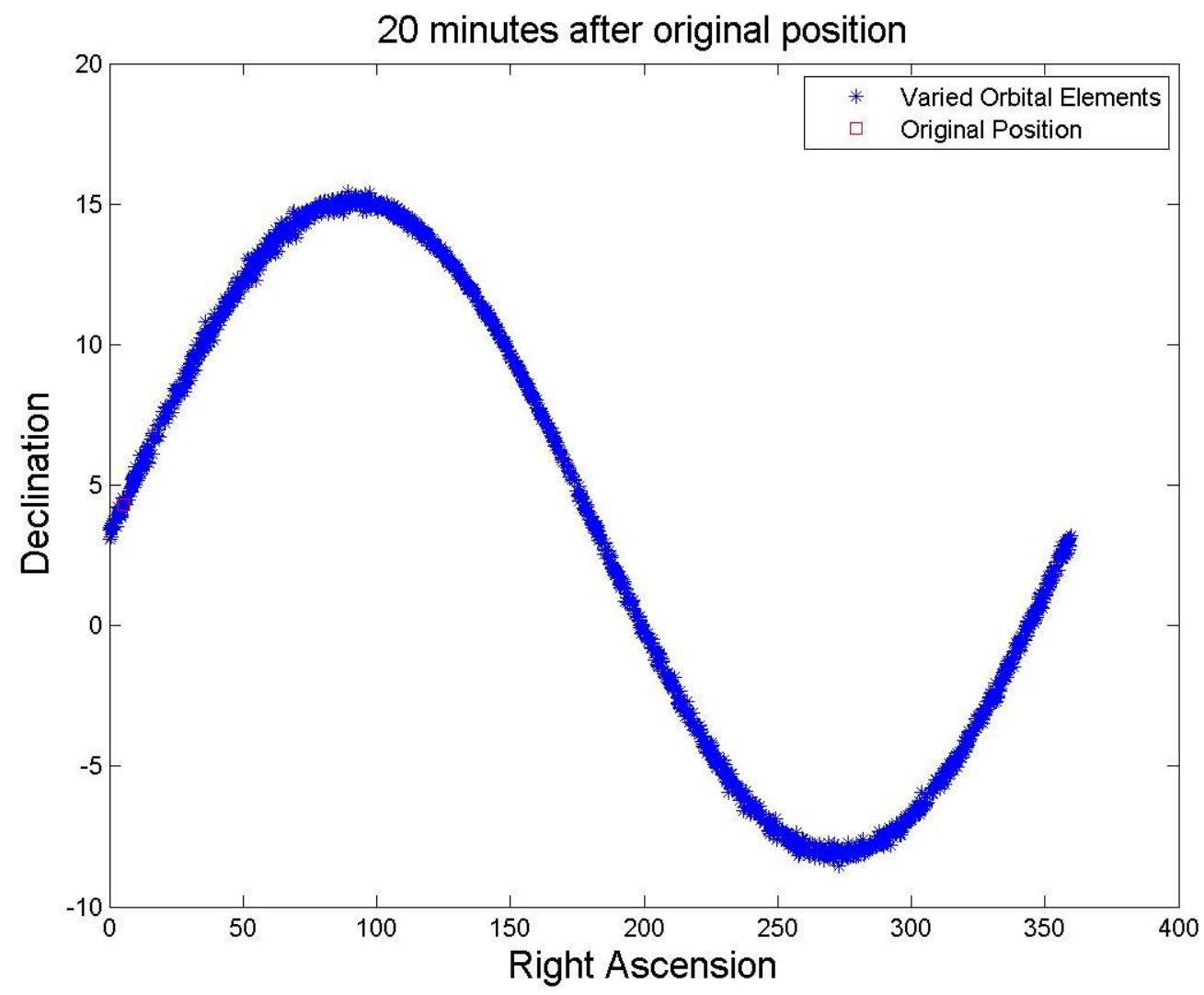



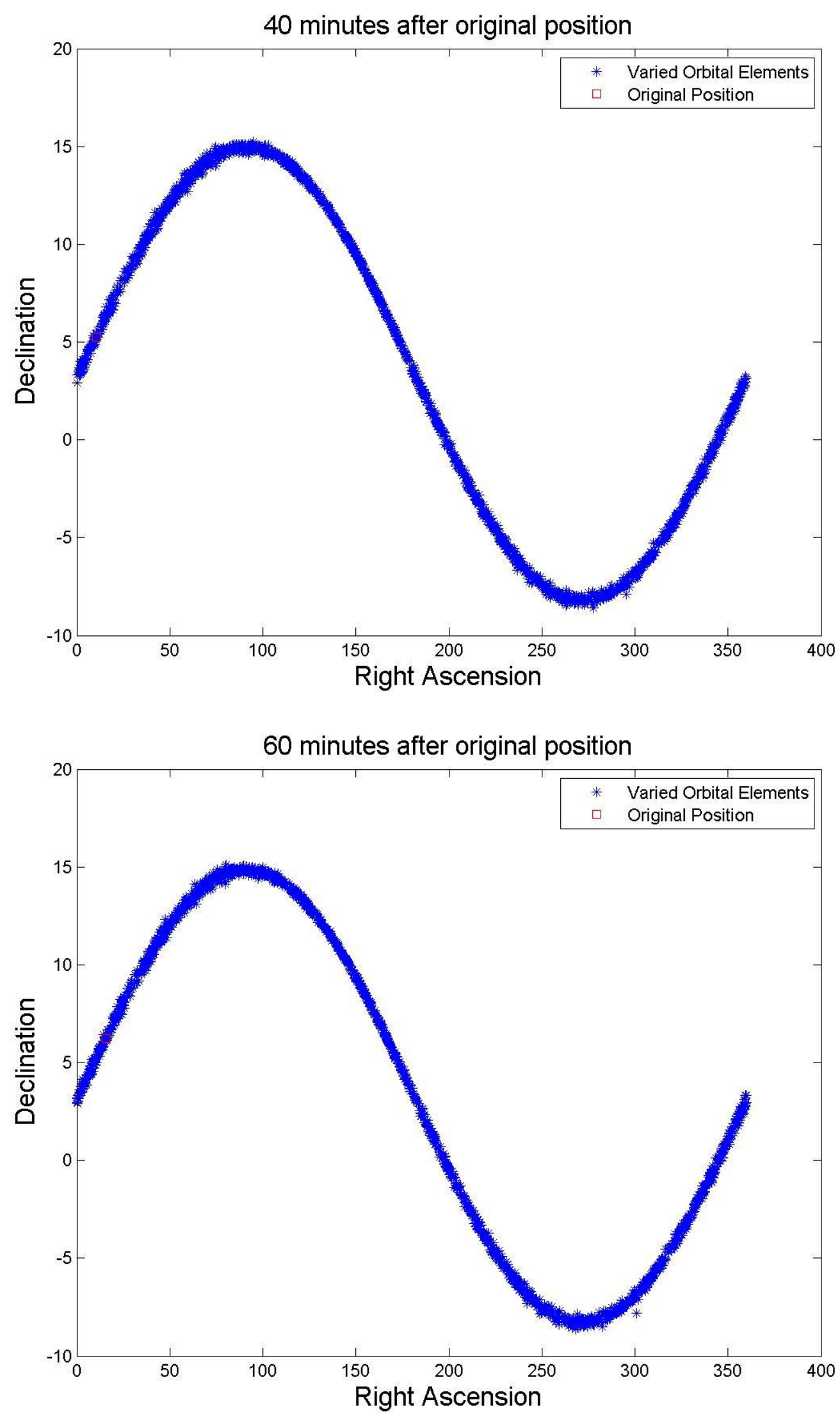


\section{Object 20070780002}

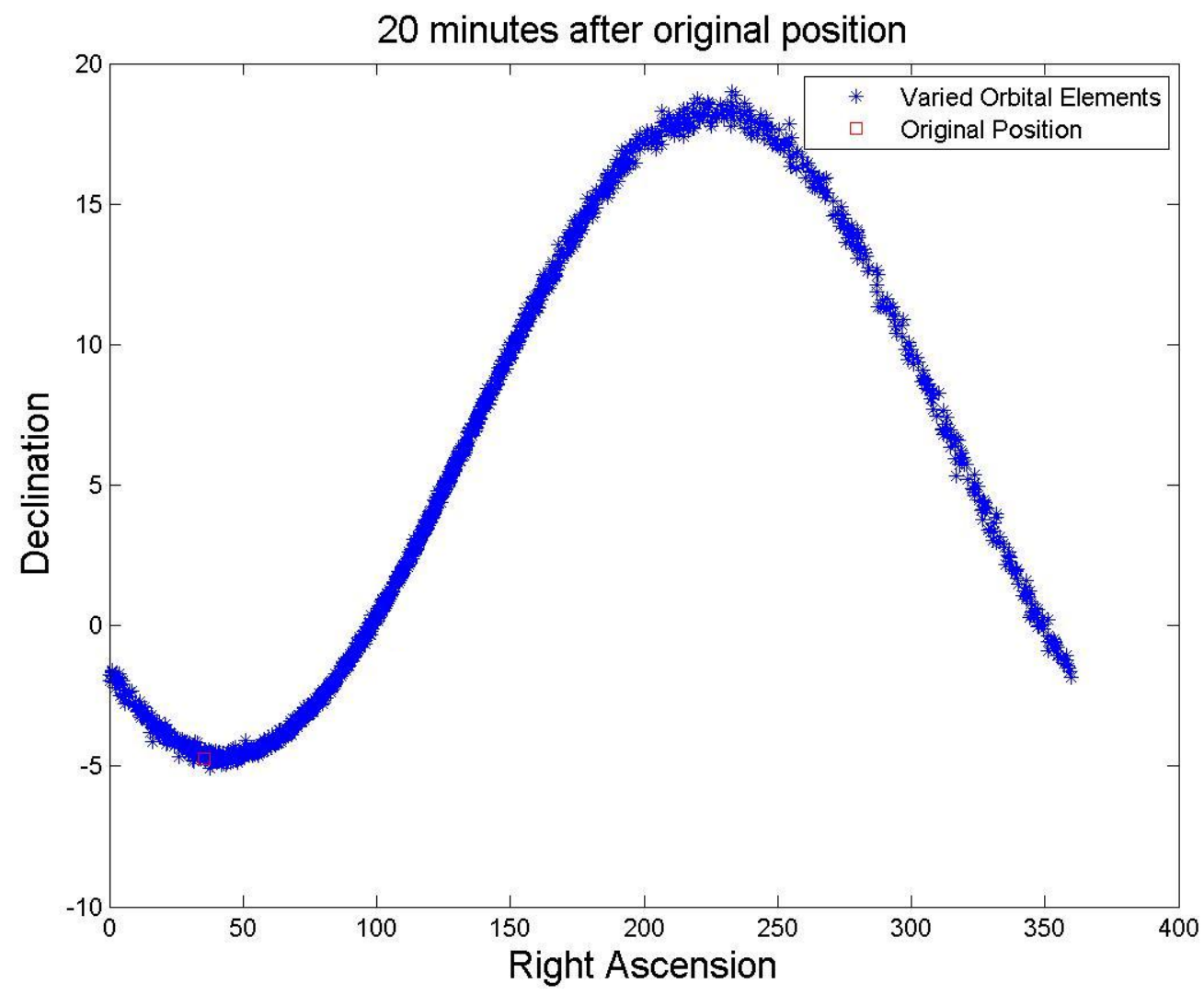



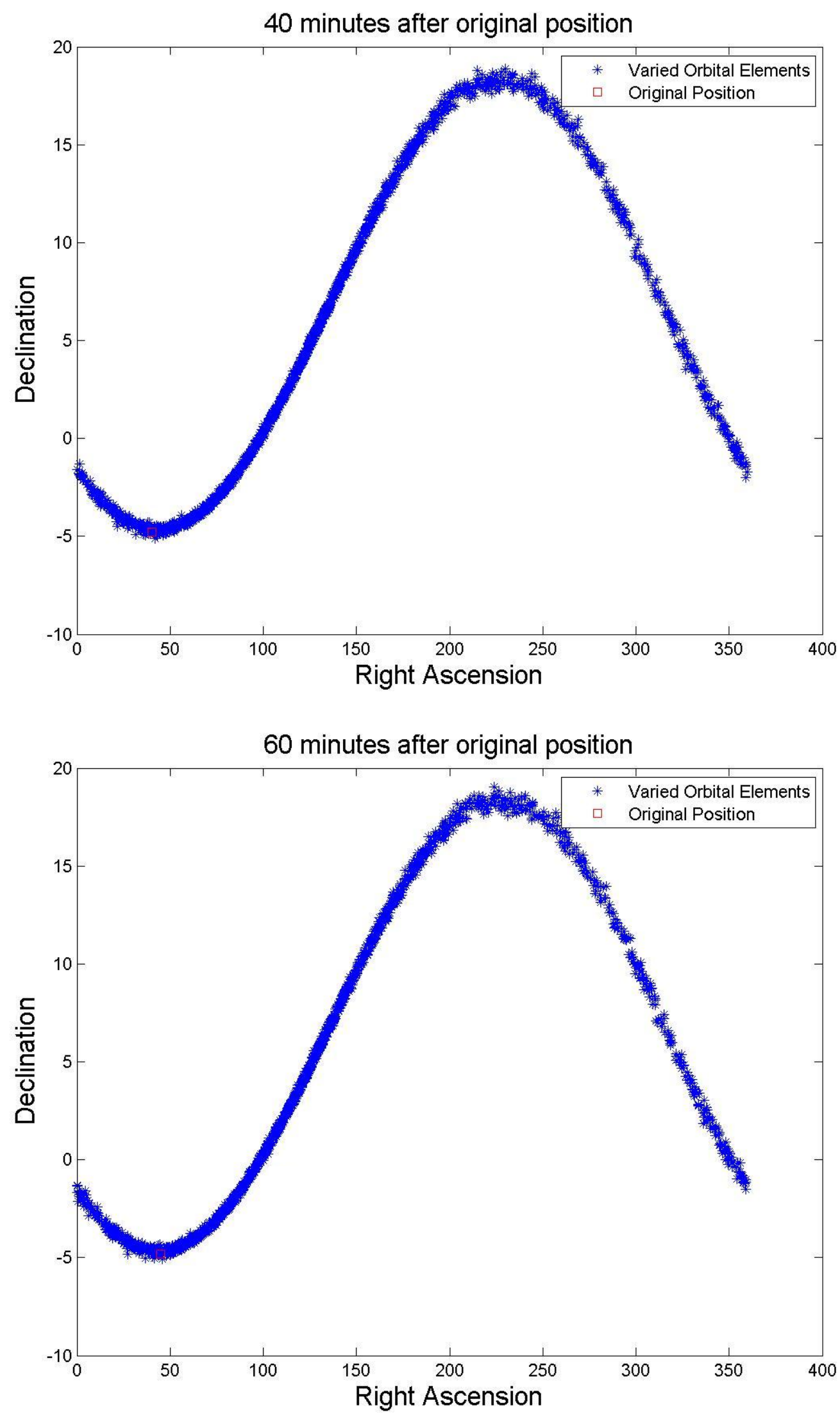


\section{Object 20070790001}

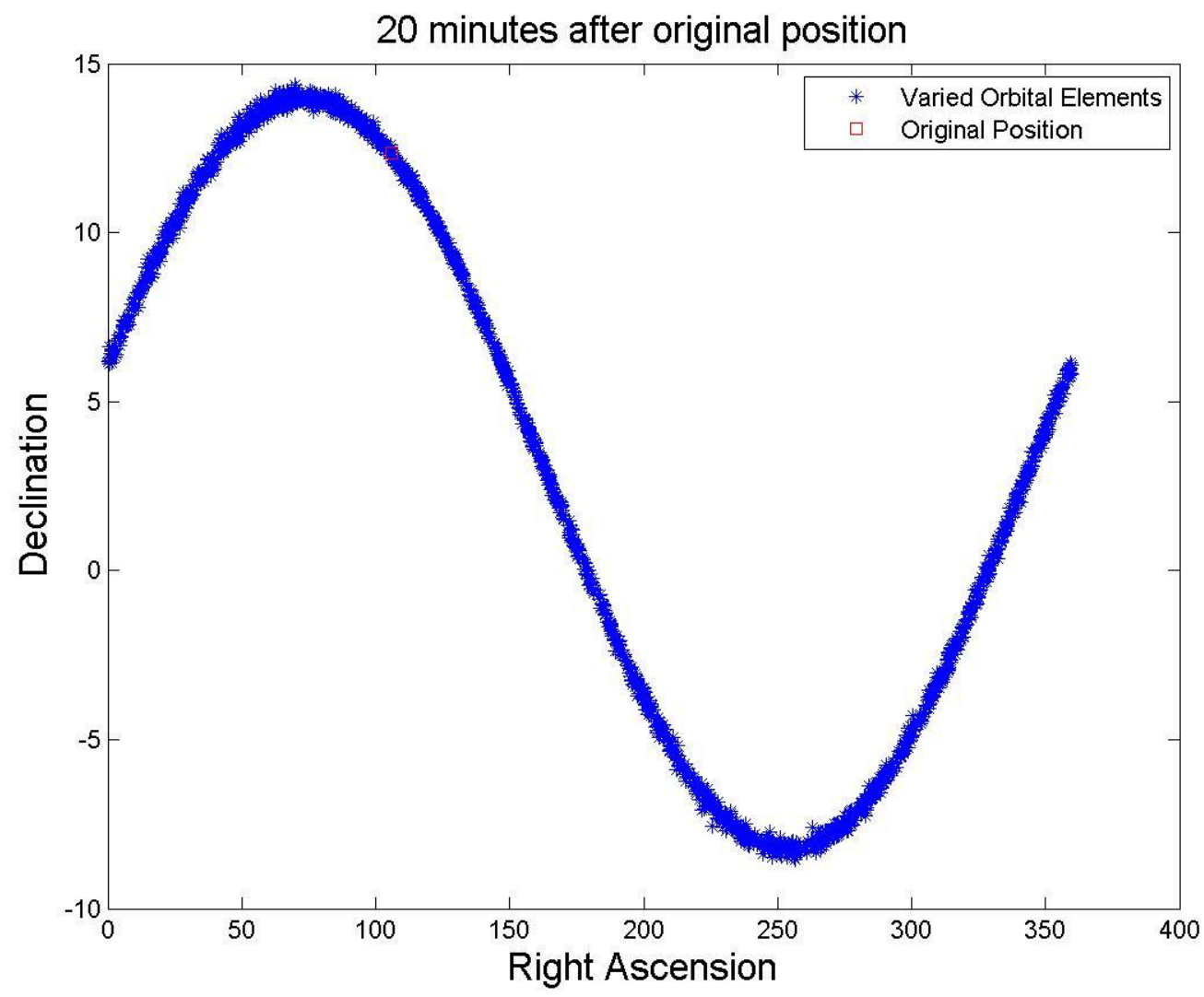



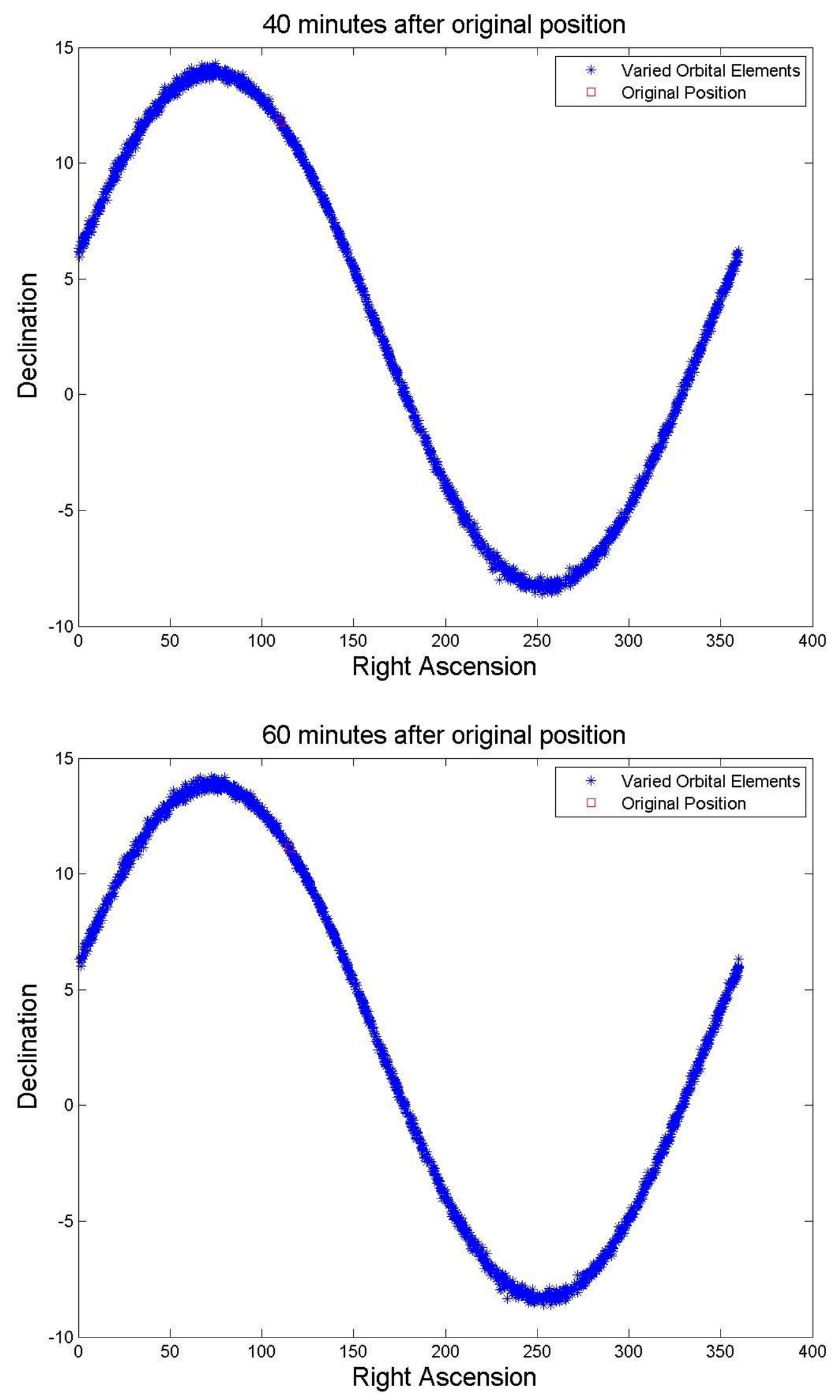


\section{Object 20070790001b}

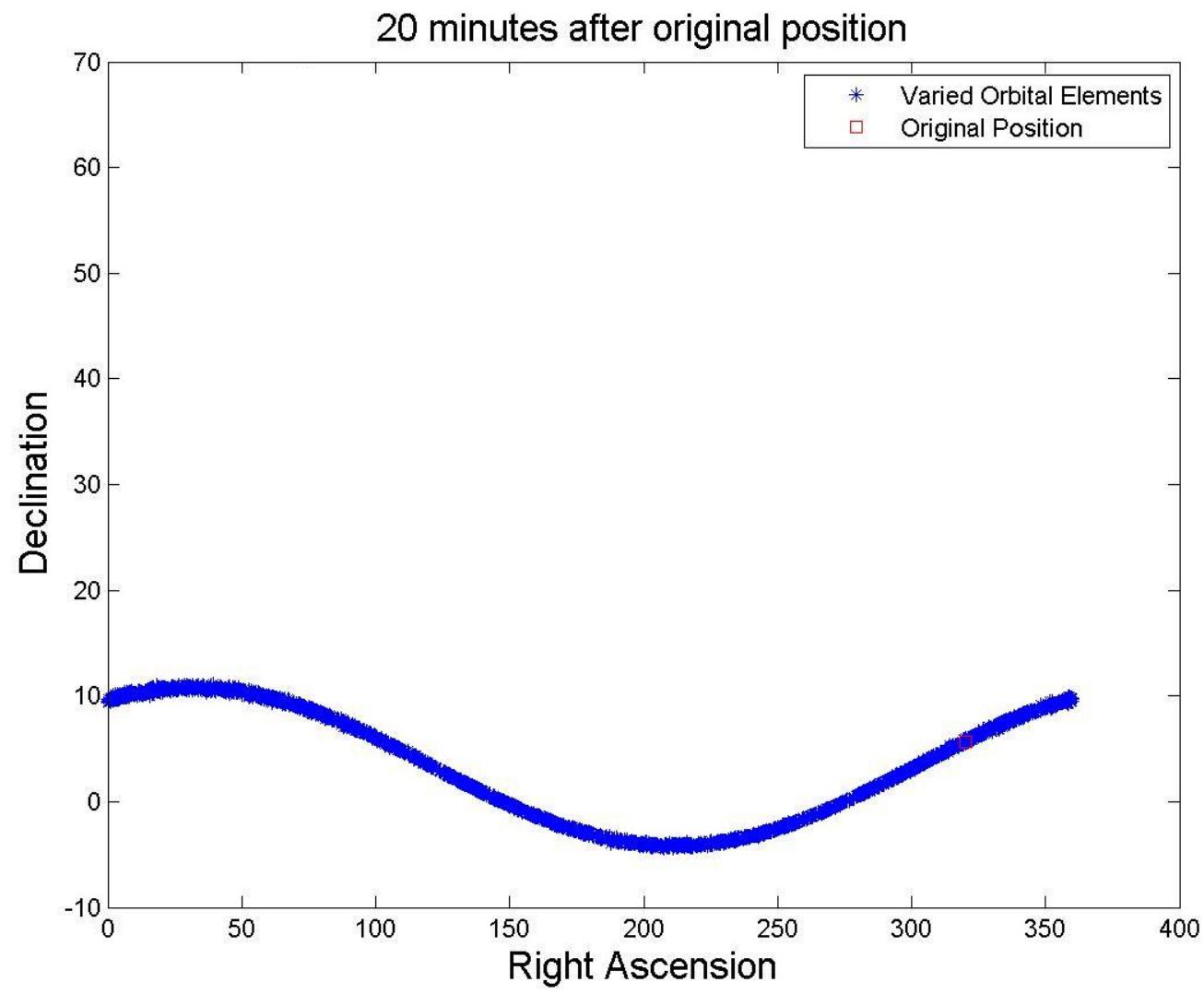



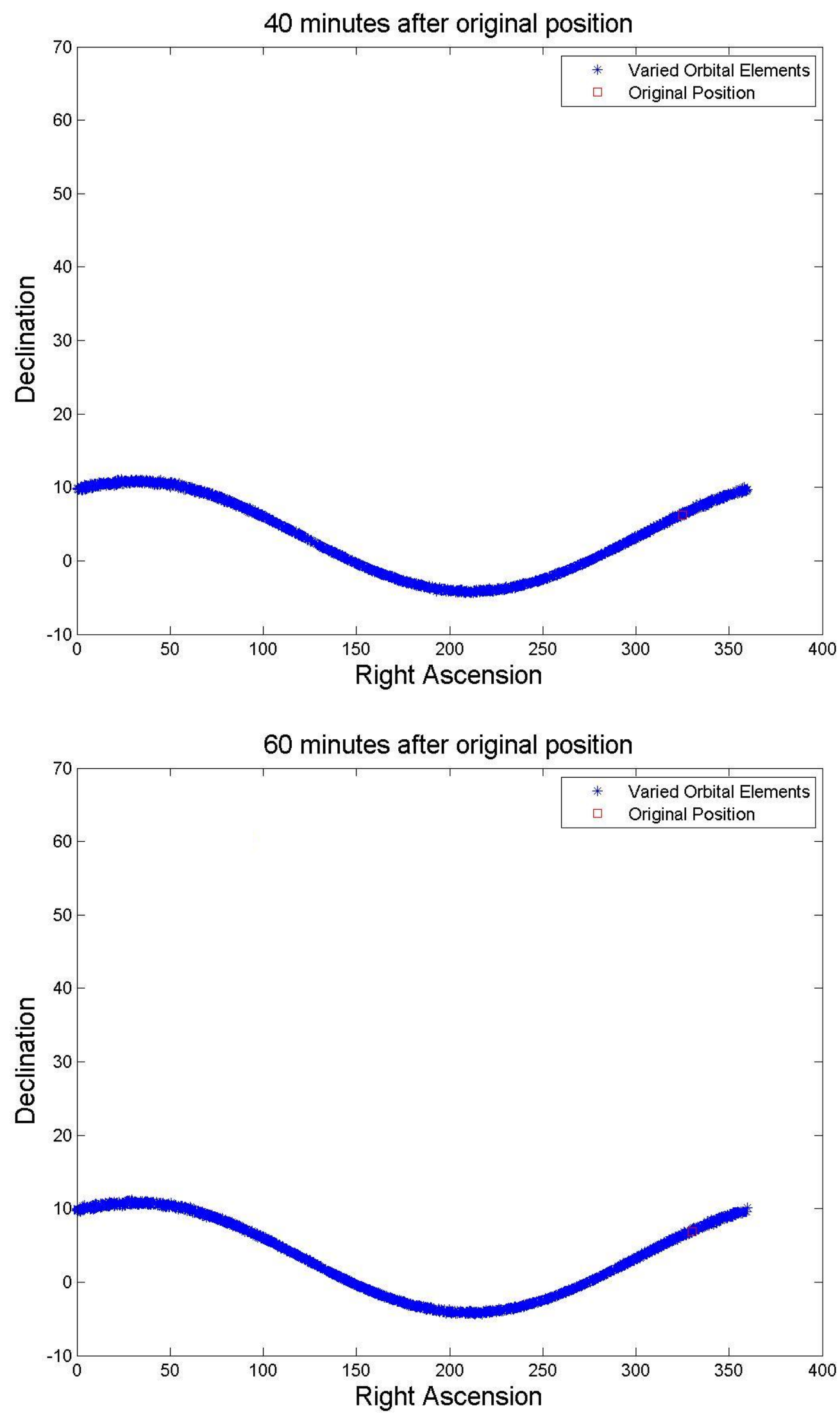


\section{Object 20070800001}

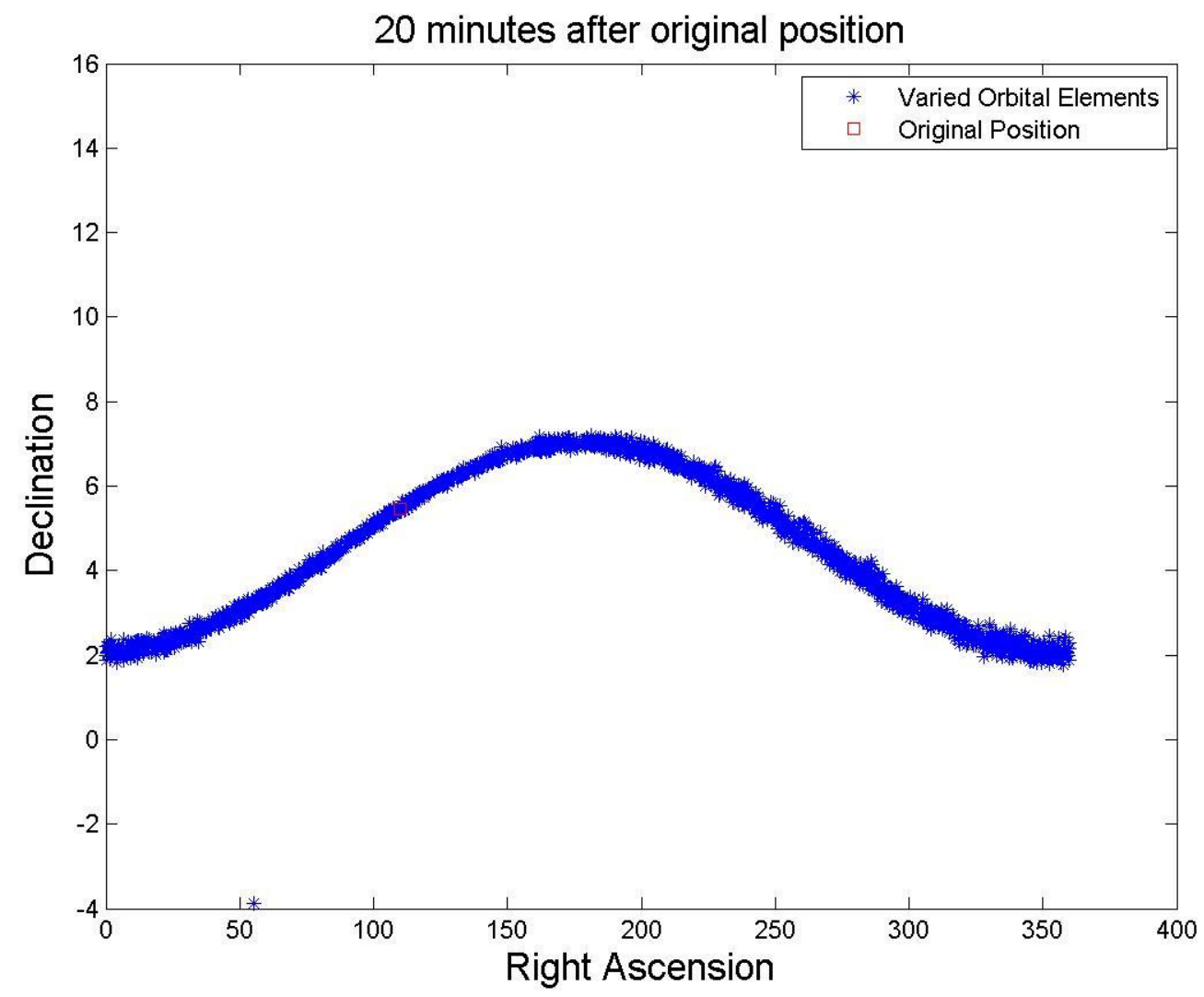



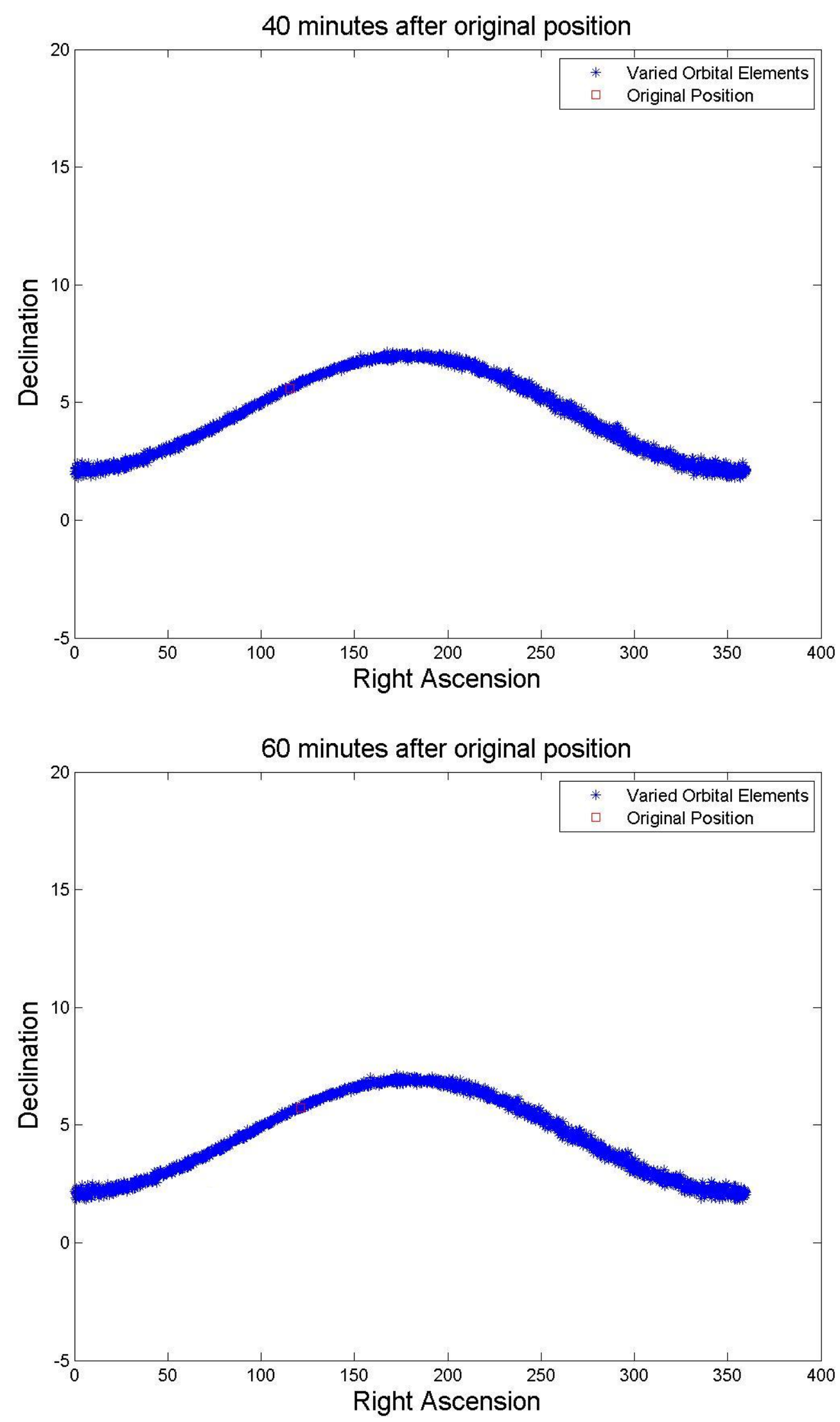


\section{Object 20070800002}

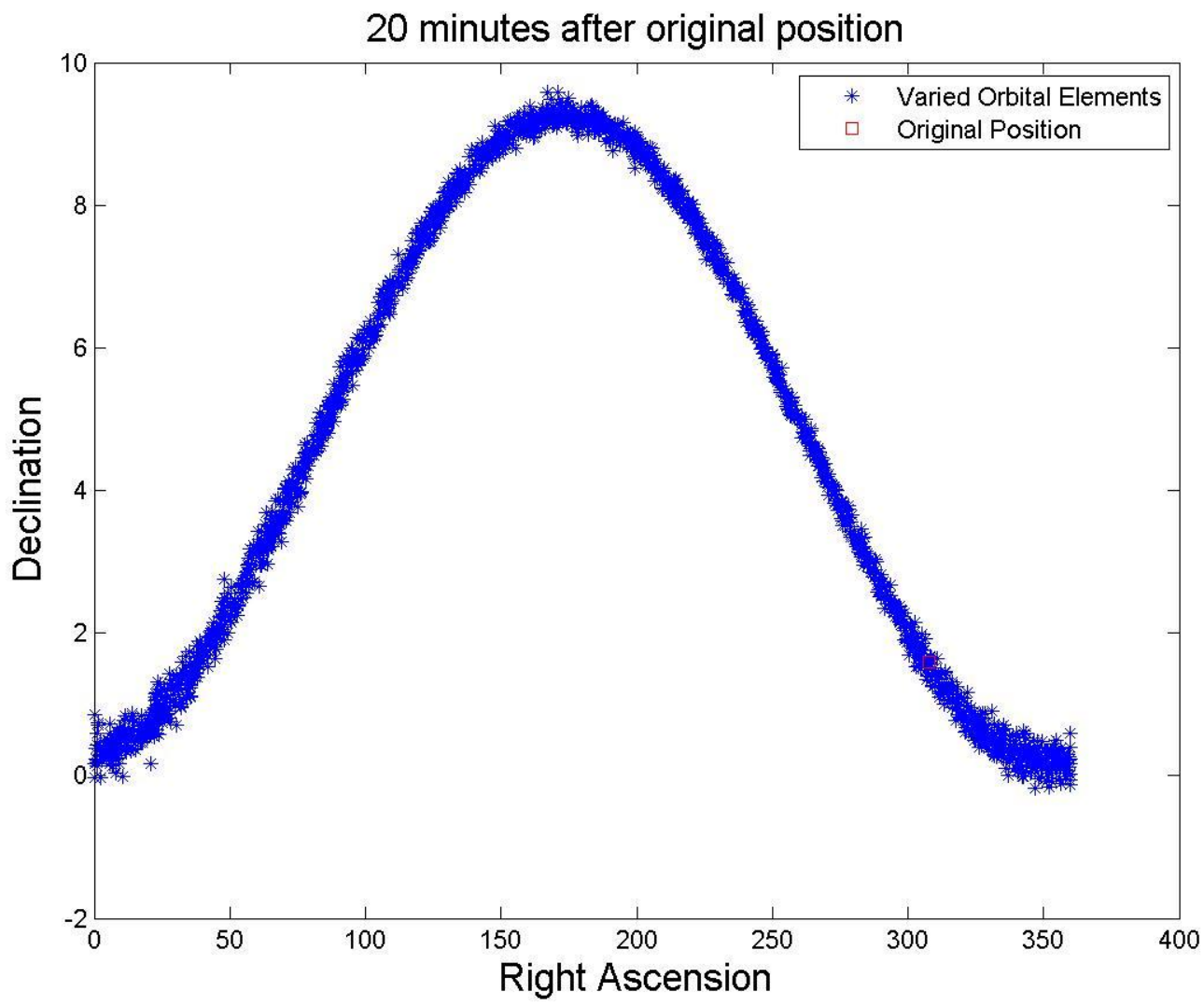



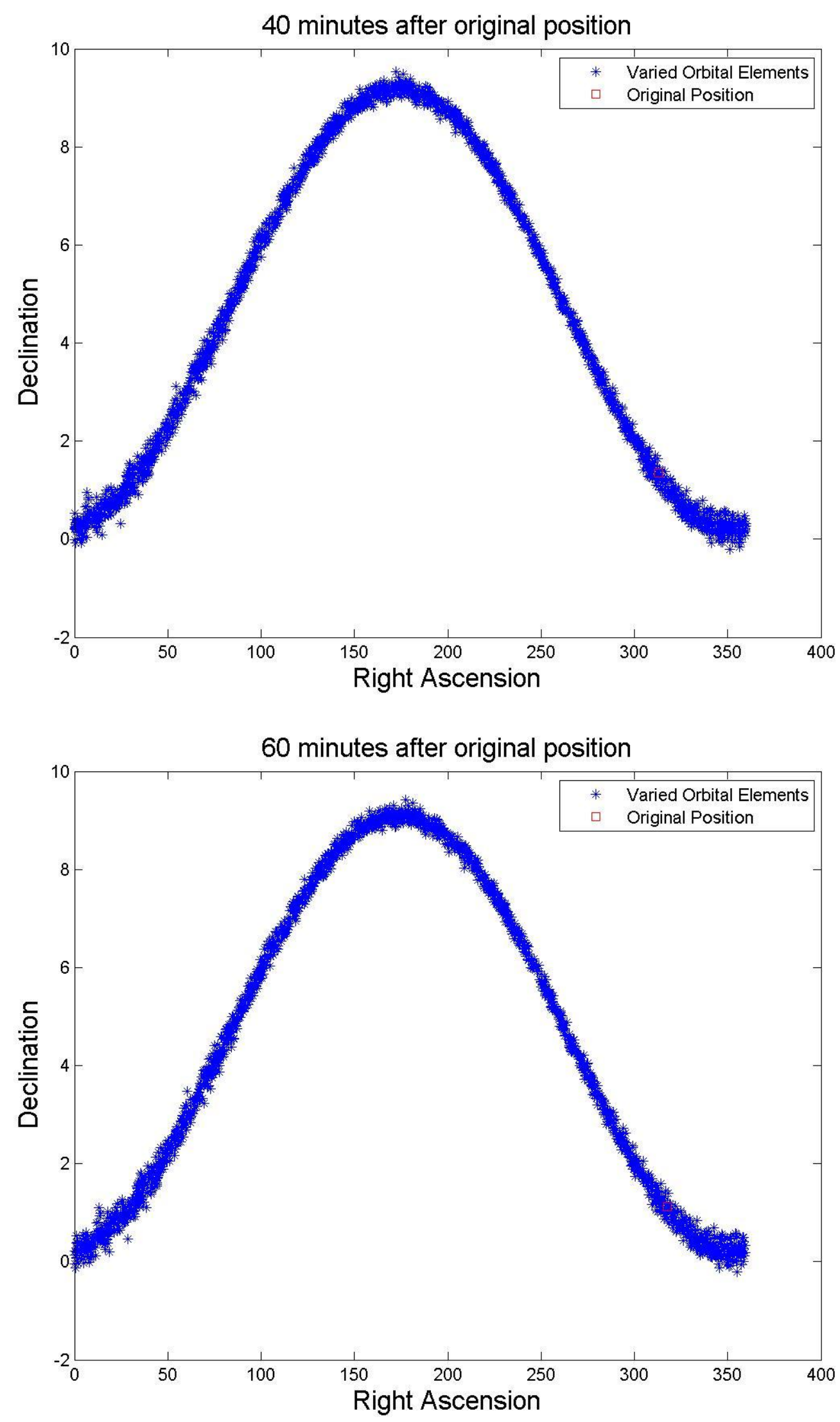


\section{Object 20070800008}

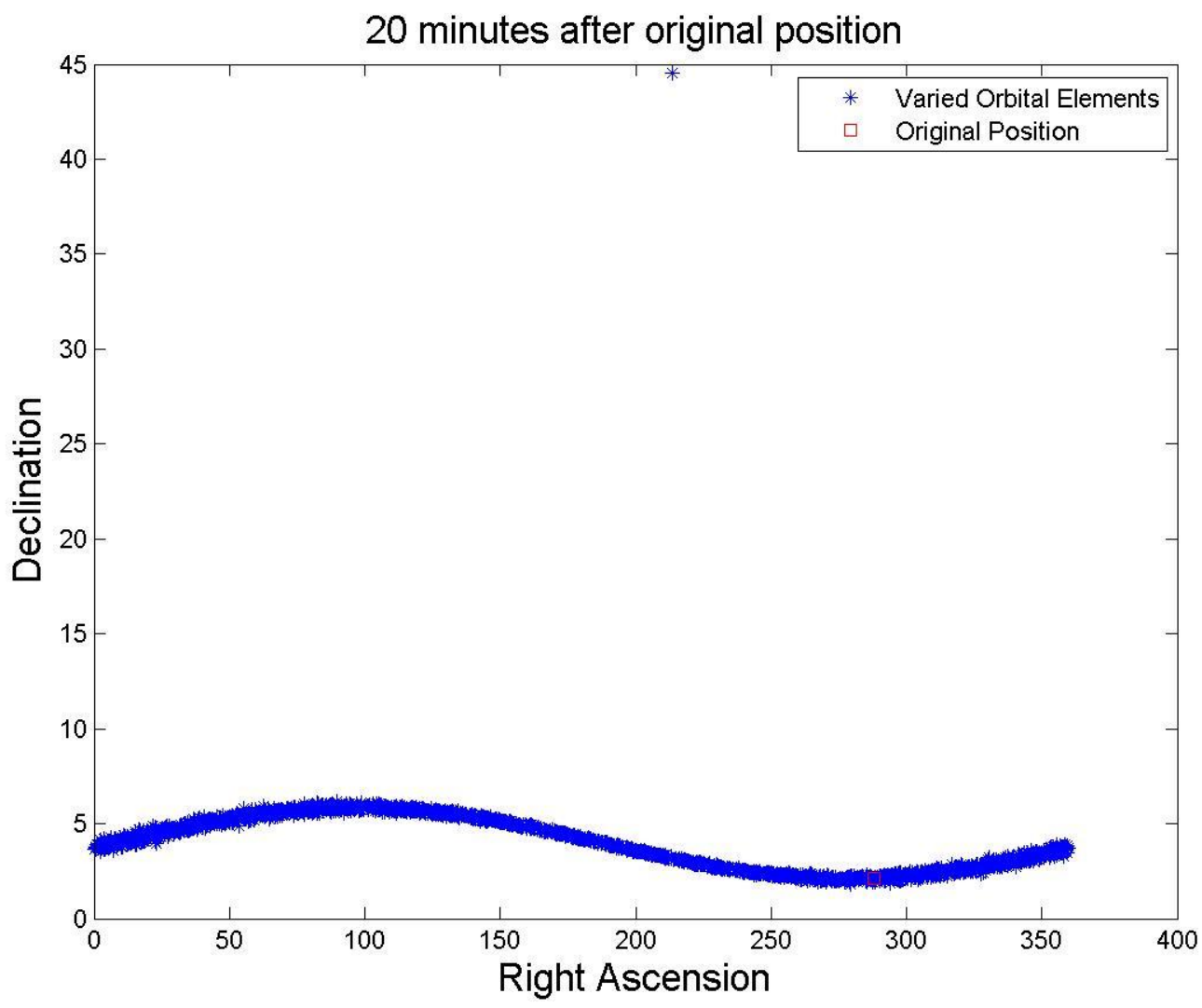



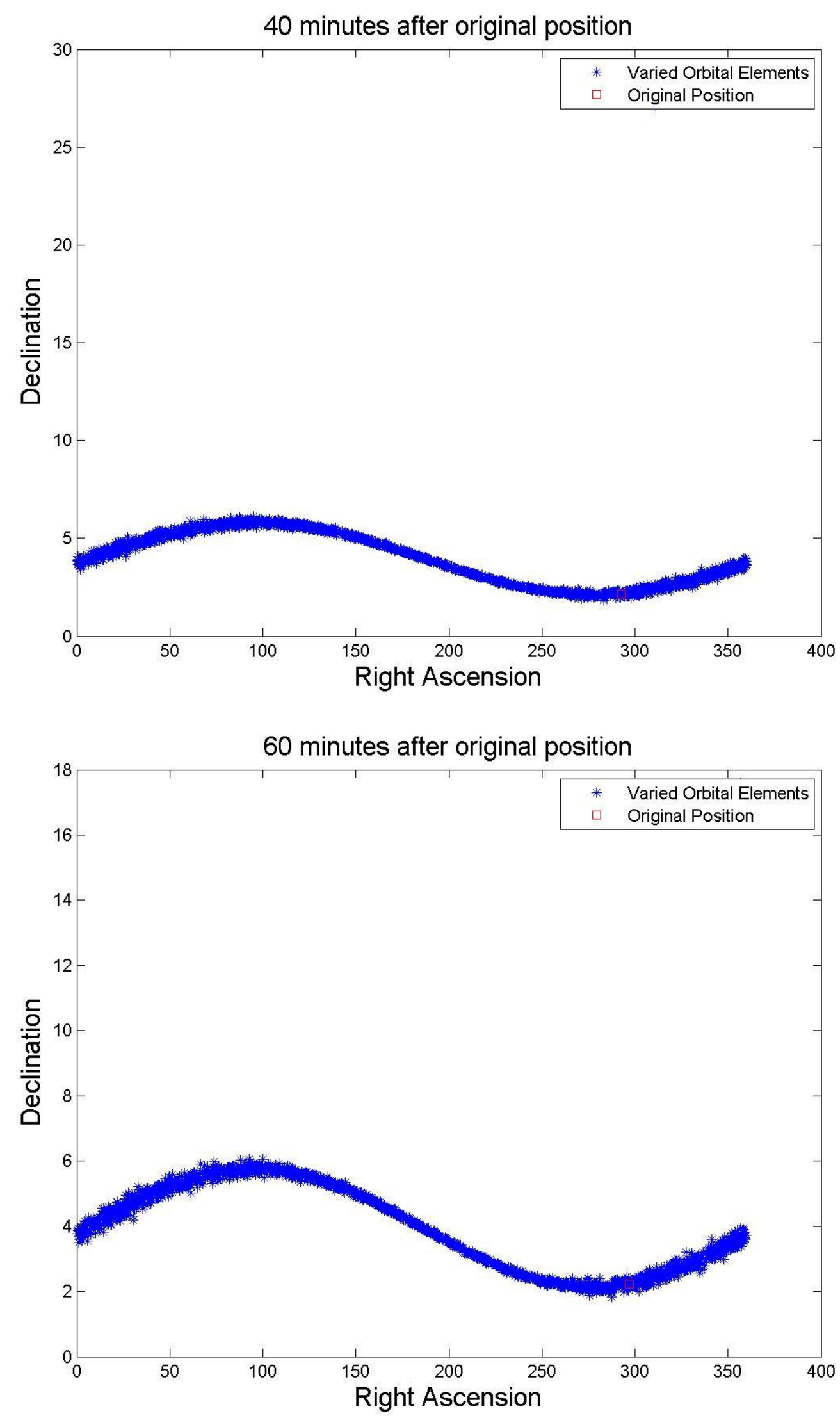


\section{Object 20070810001}

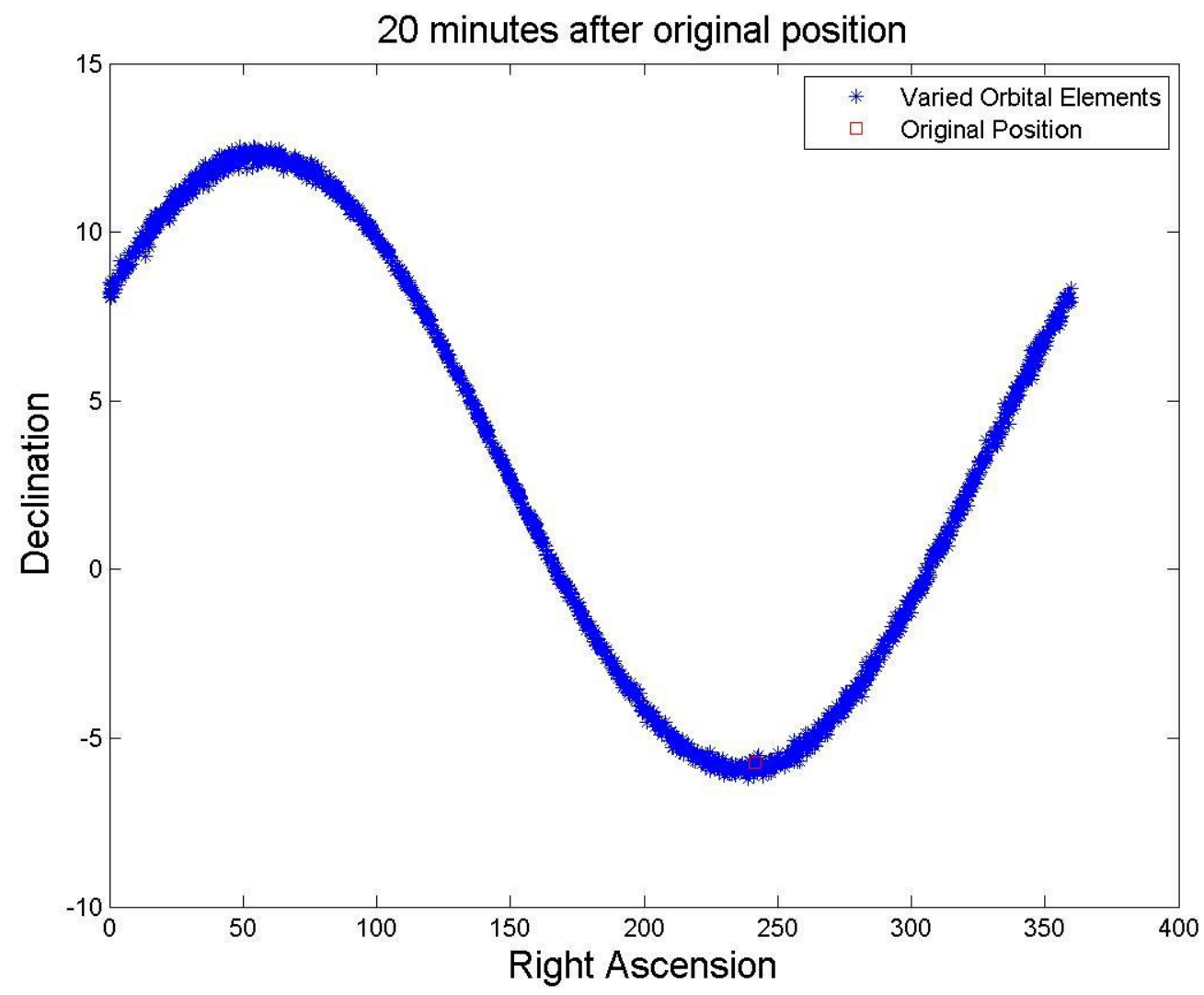



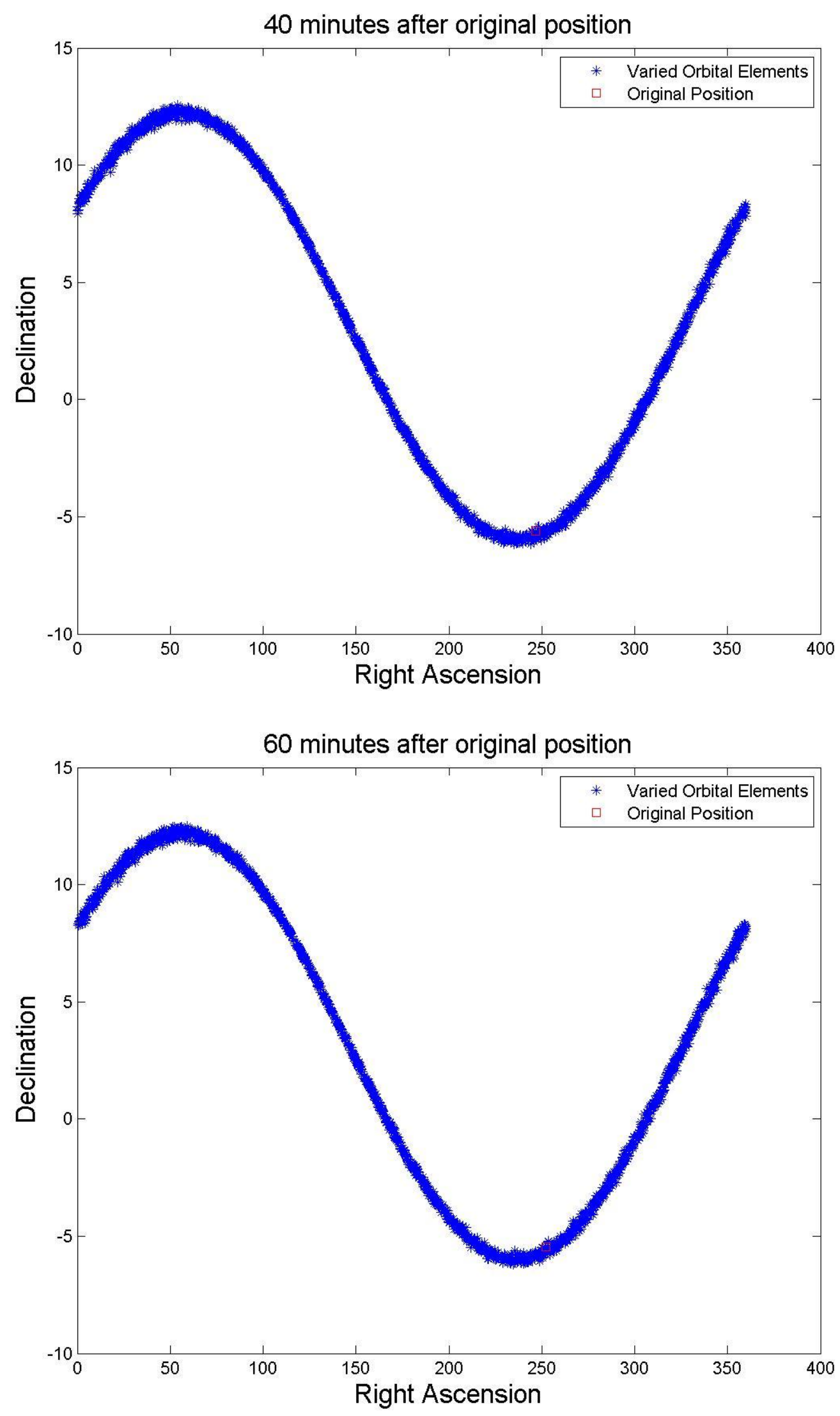


\section{Object 20070810002}

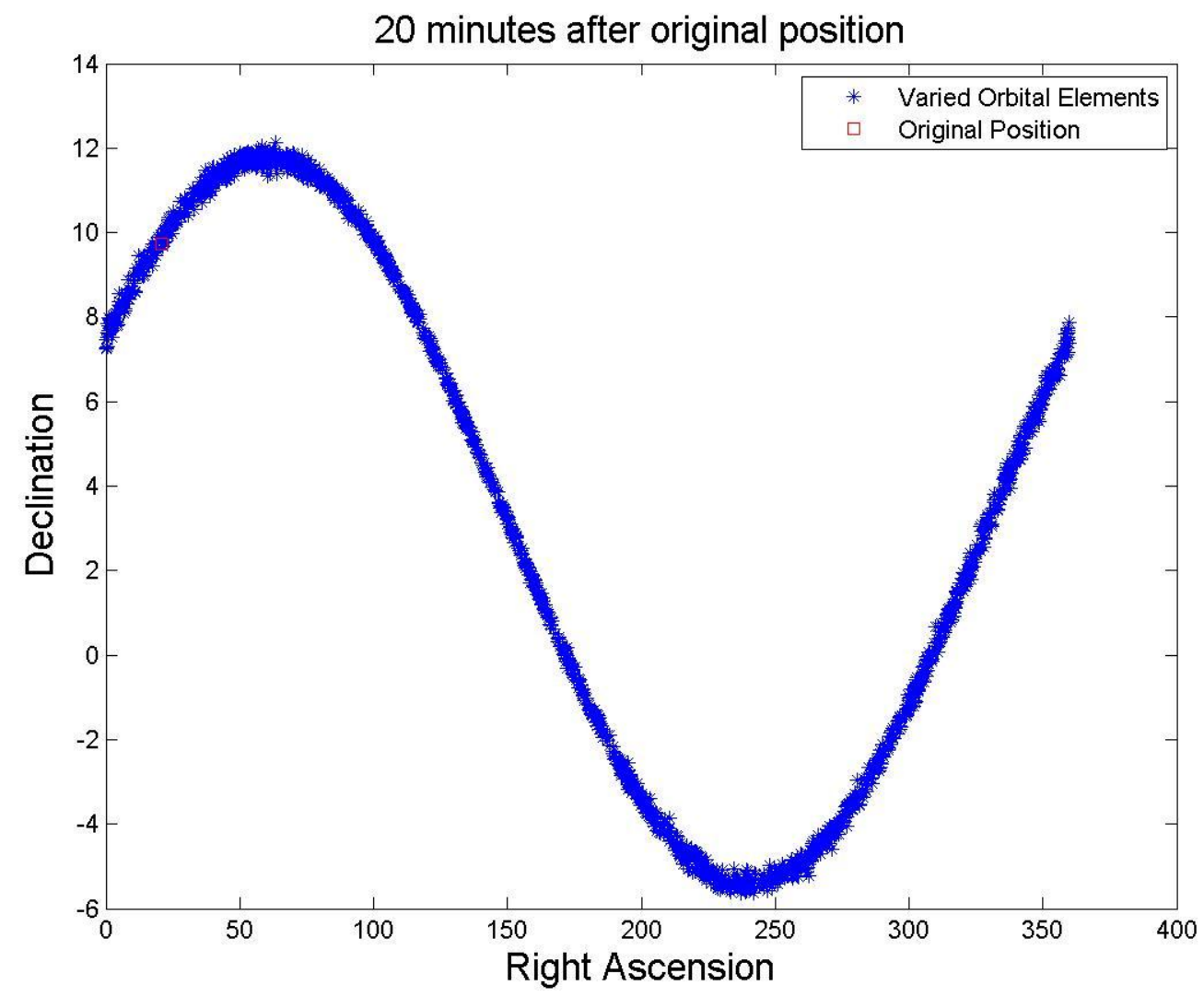



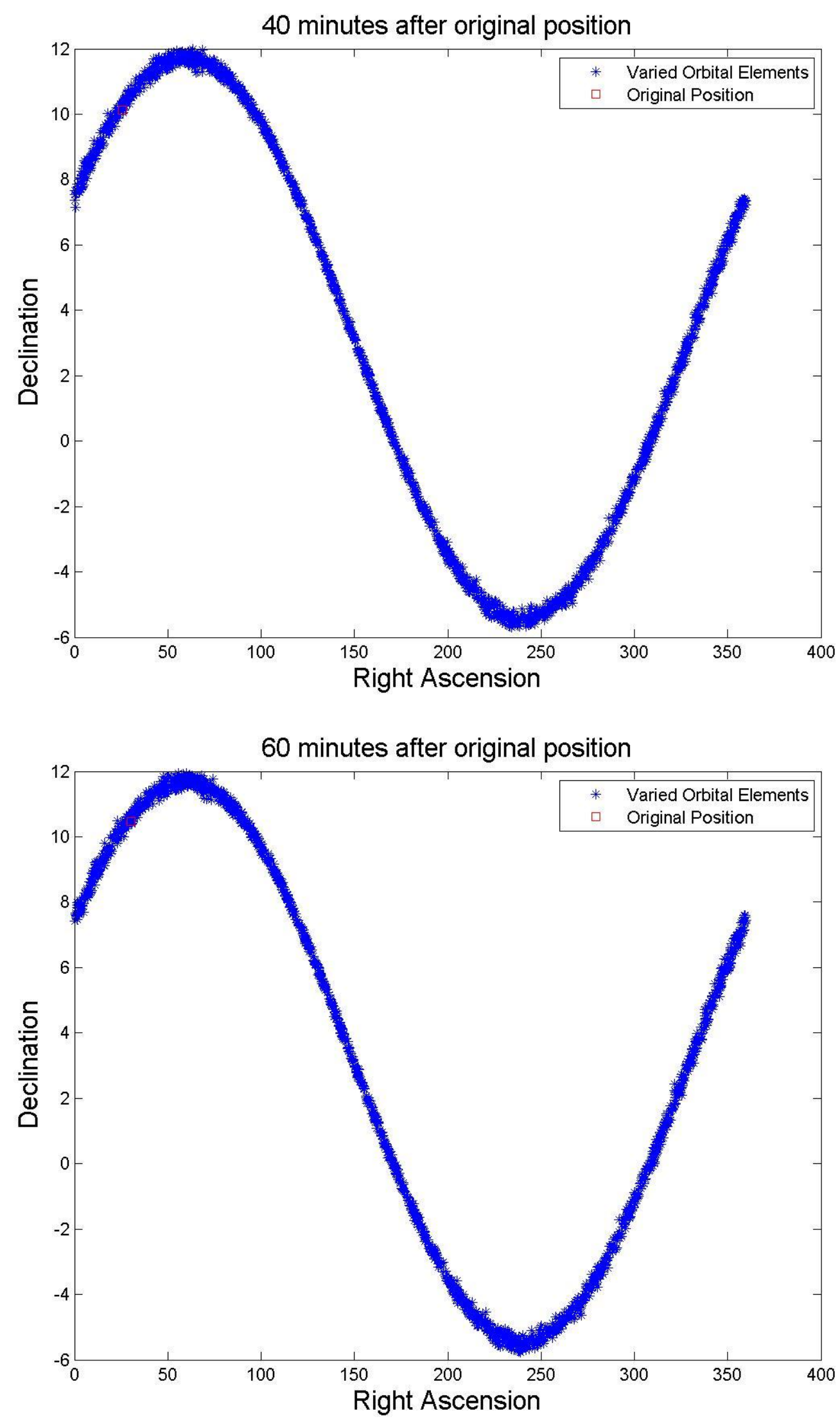


\section{Object 20070810003}

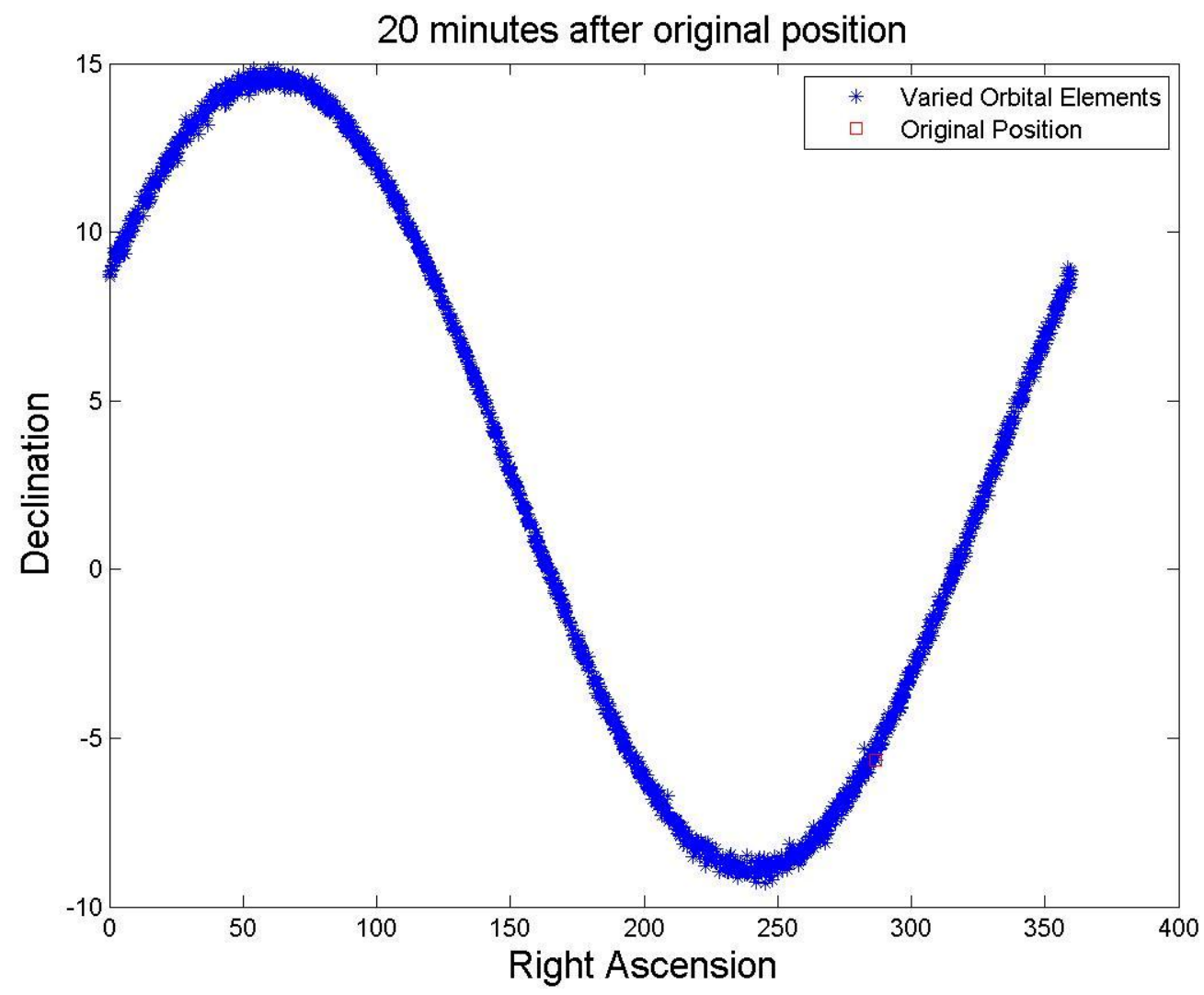



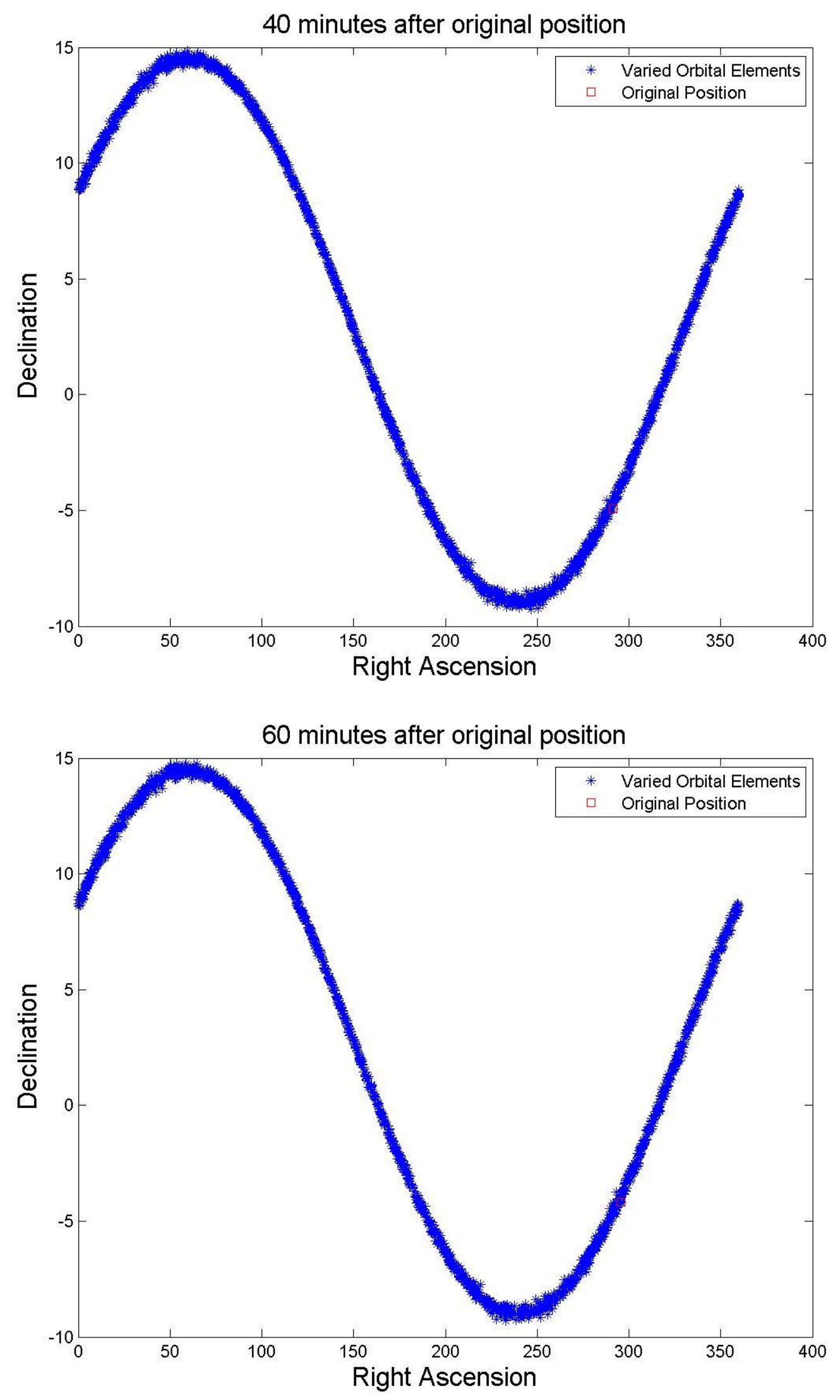


\section{Object 20070810006}

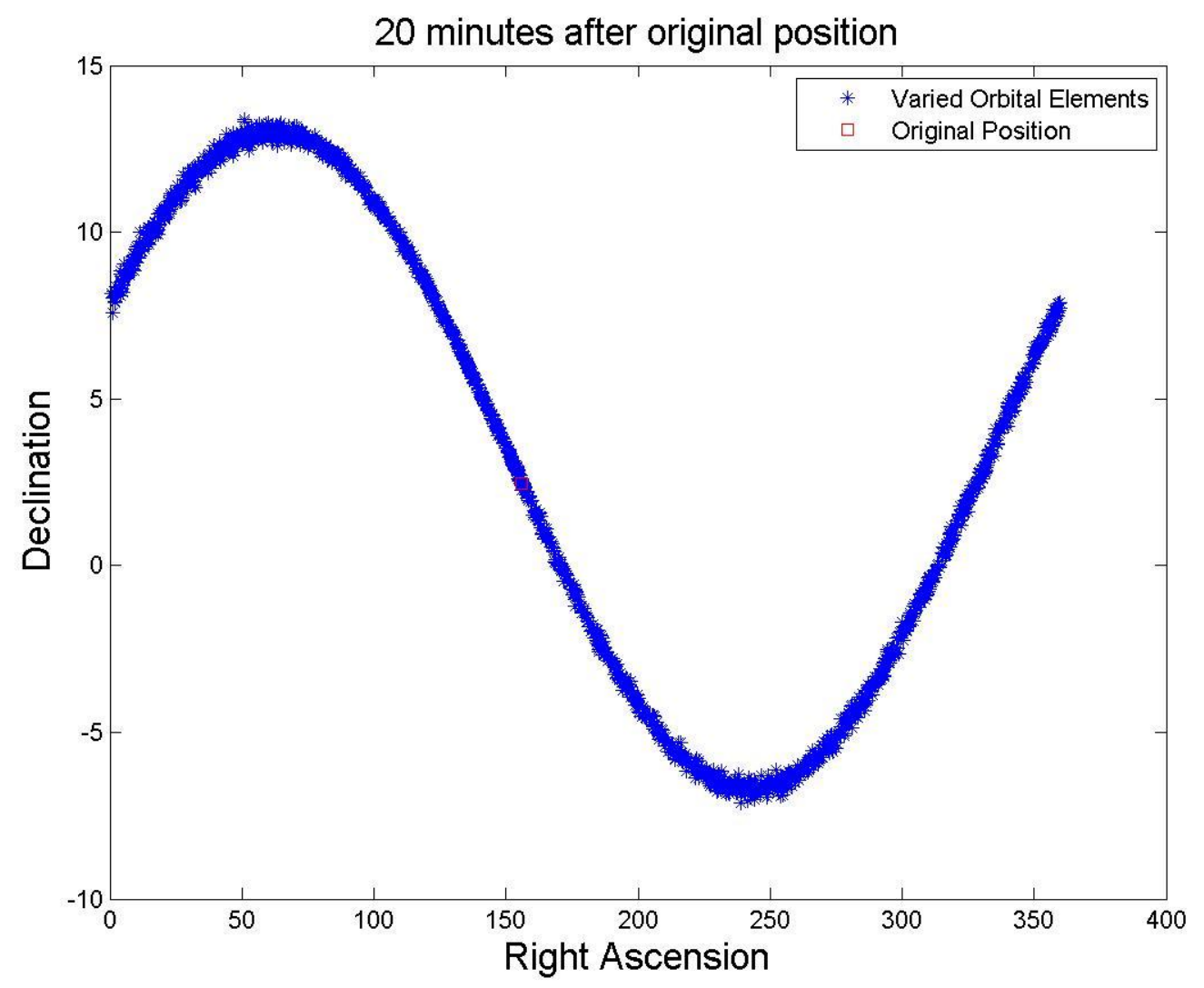



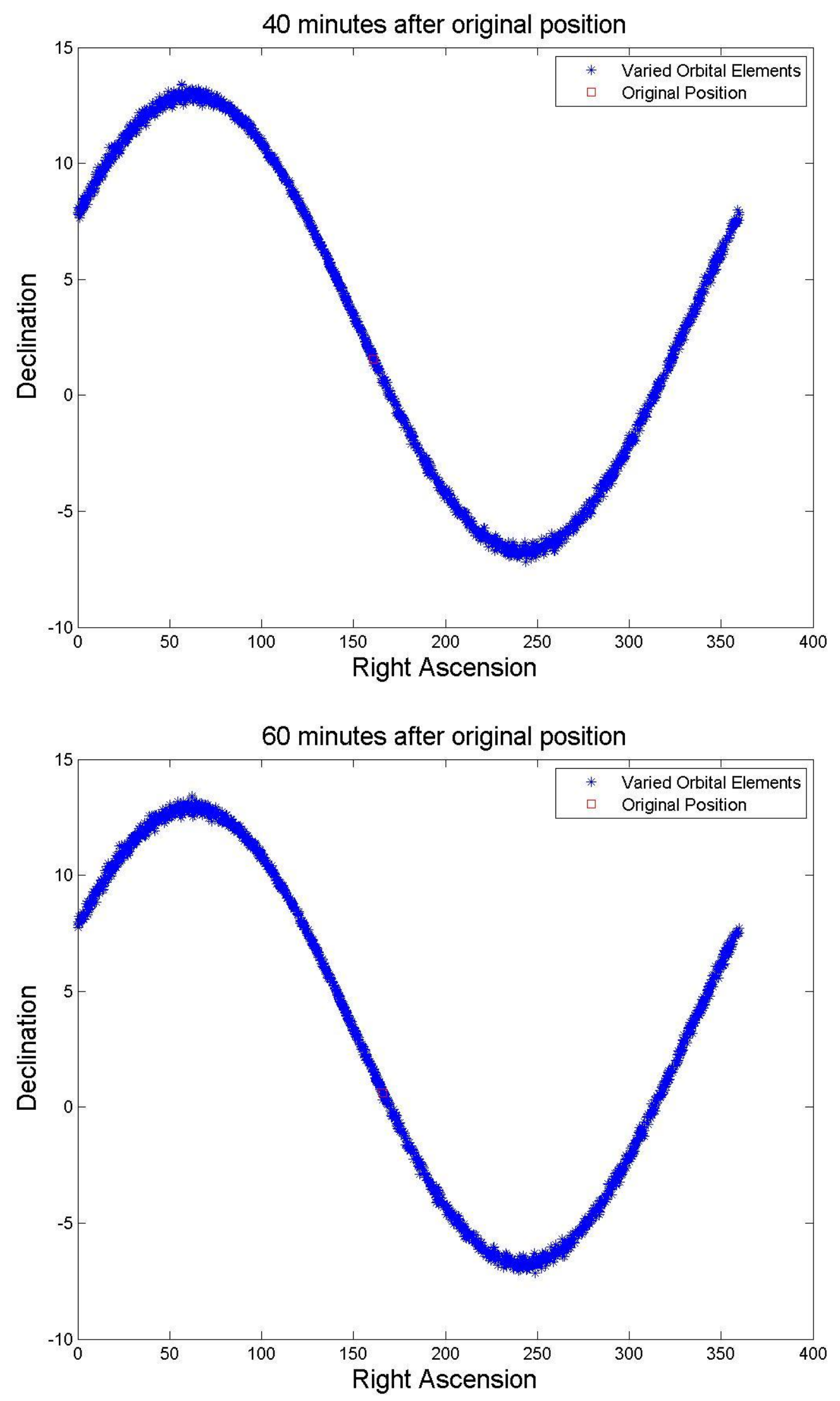


\section{Object 20070810012}

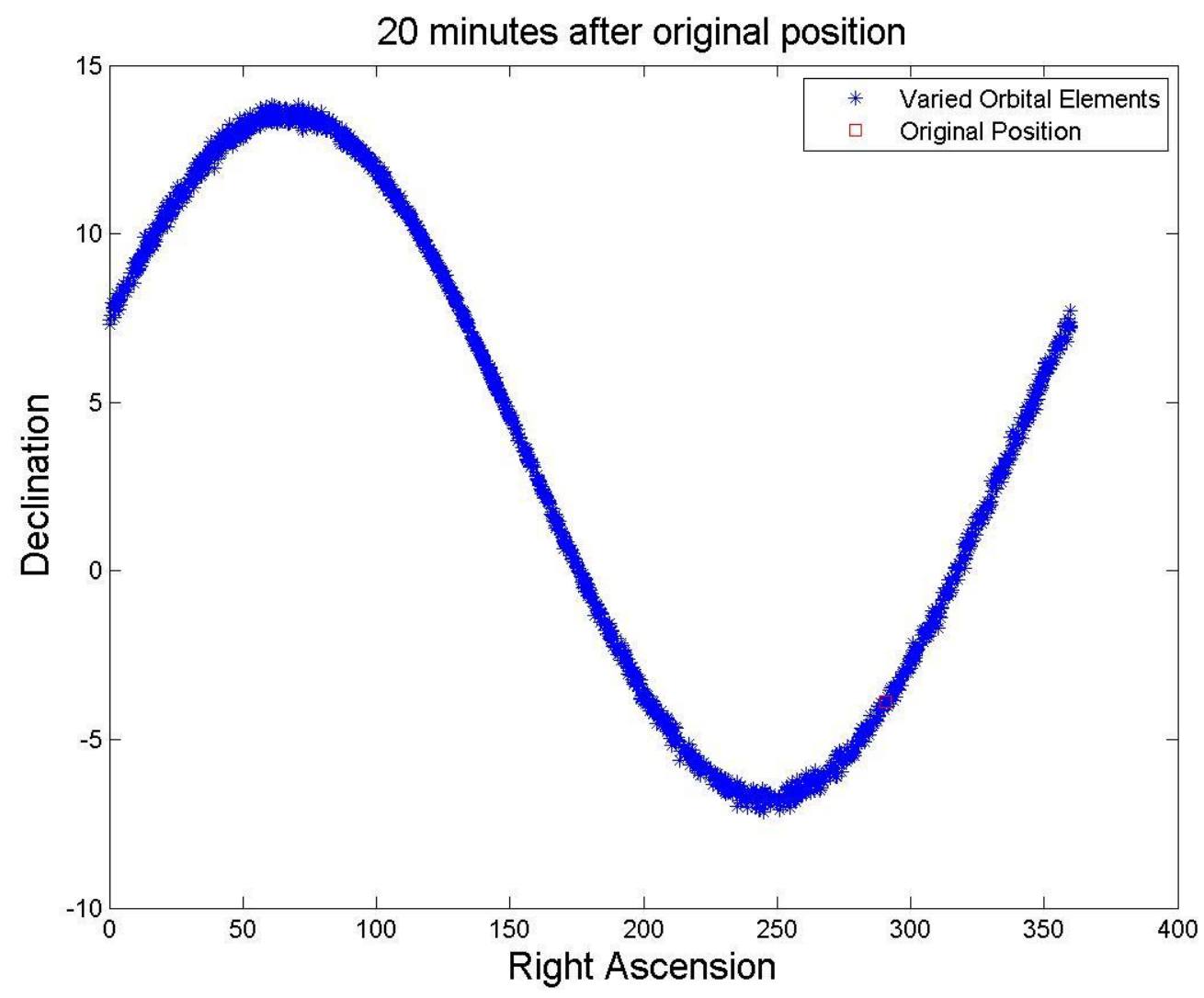



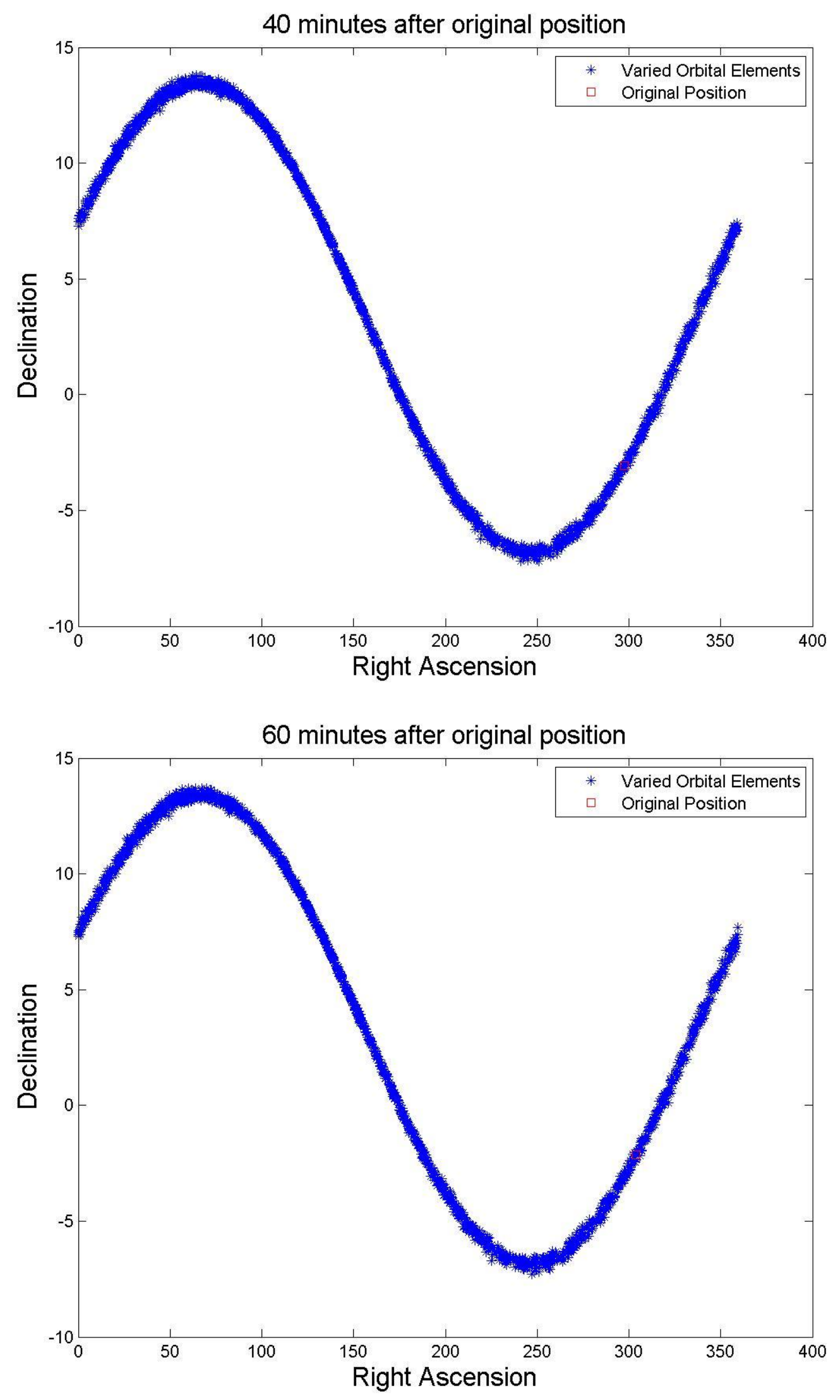


\section{Object 20070820013}

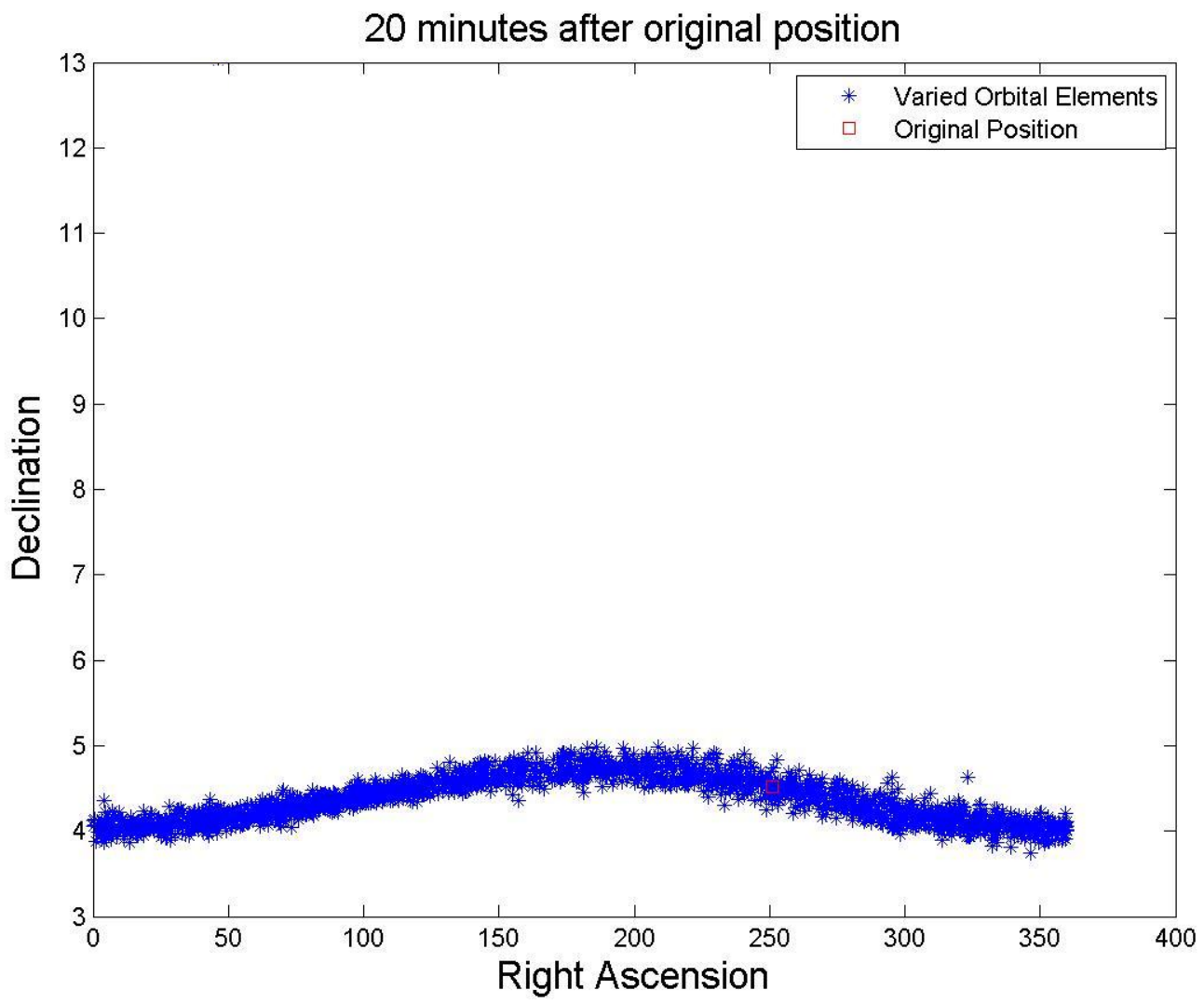



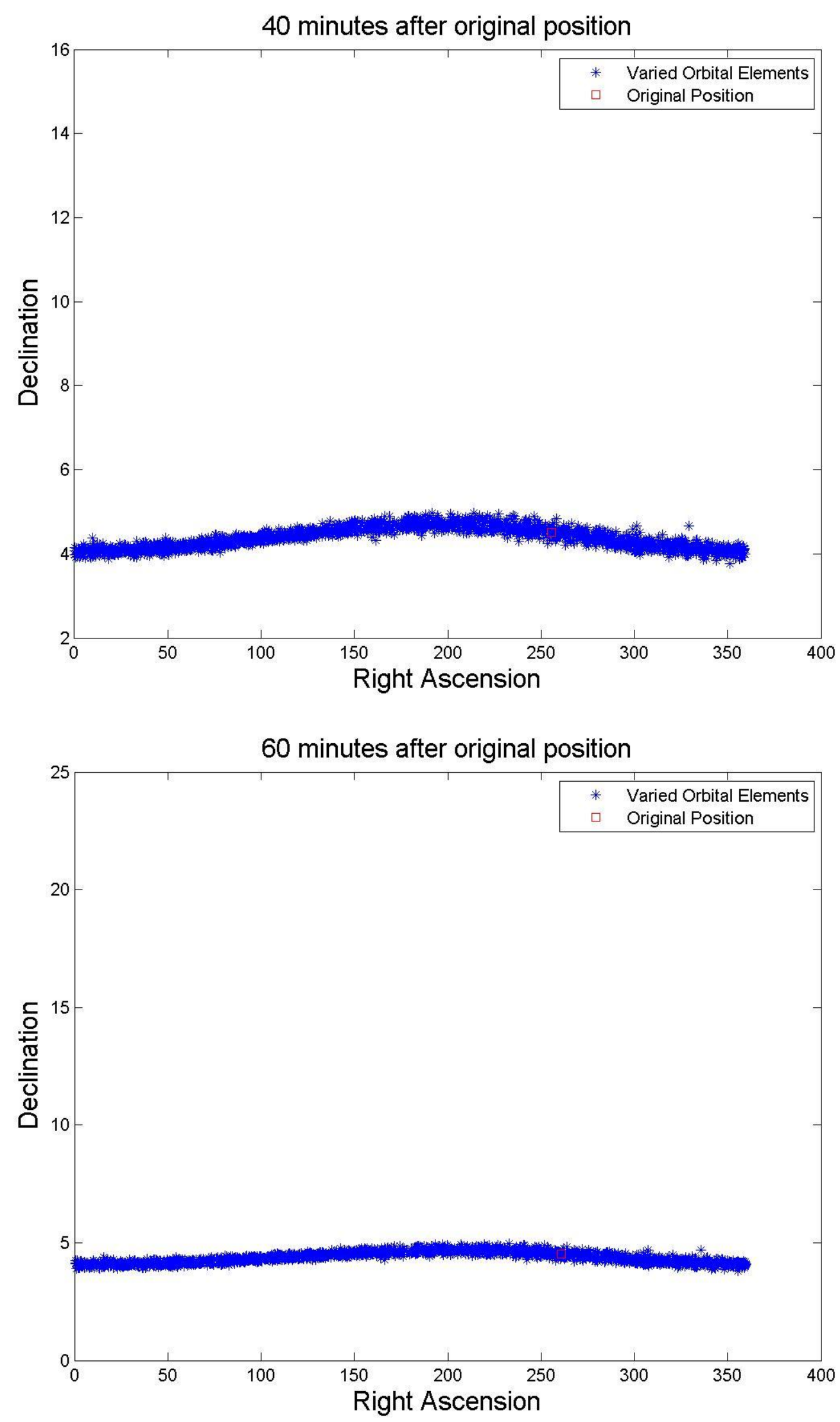


\section{Appendix C: 2008 Graphs}

Object 70301

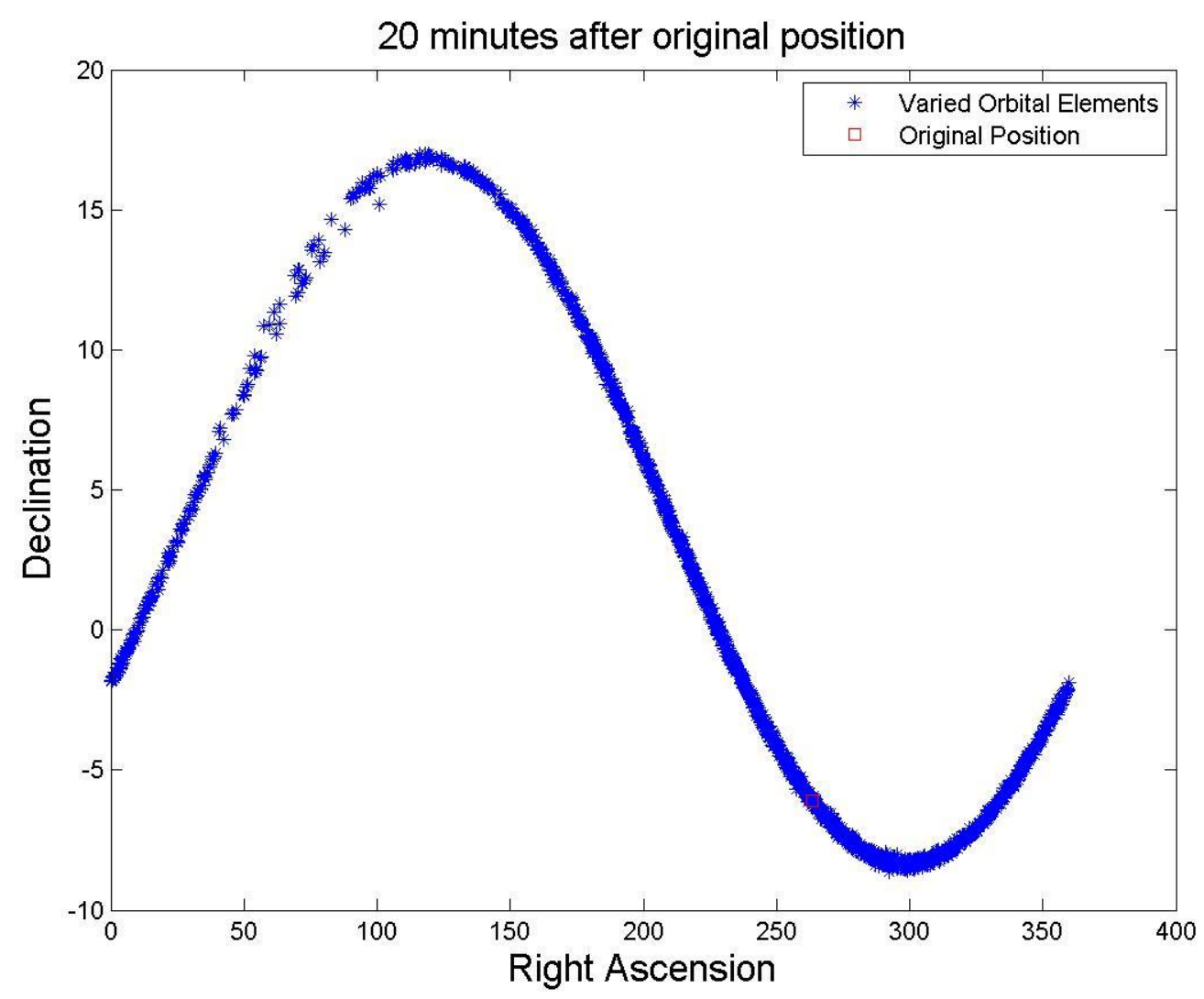



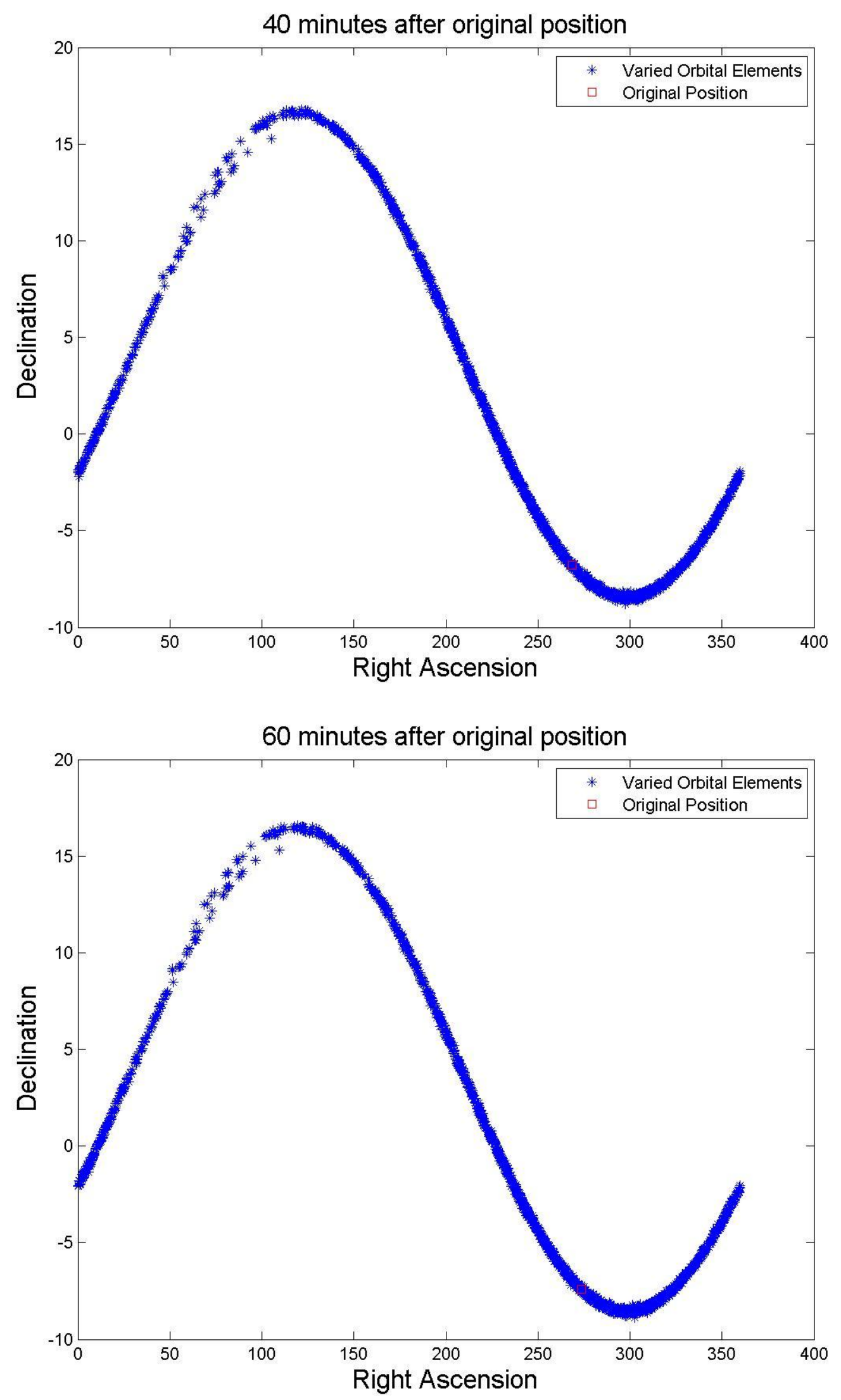


\section{Object 70302}

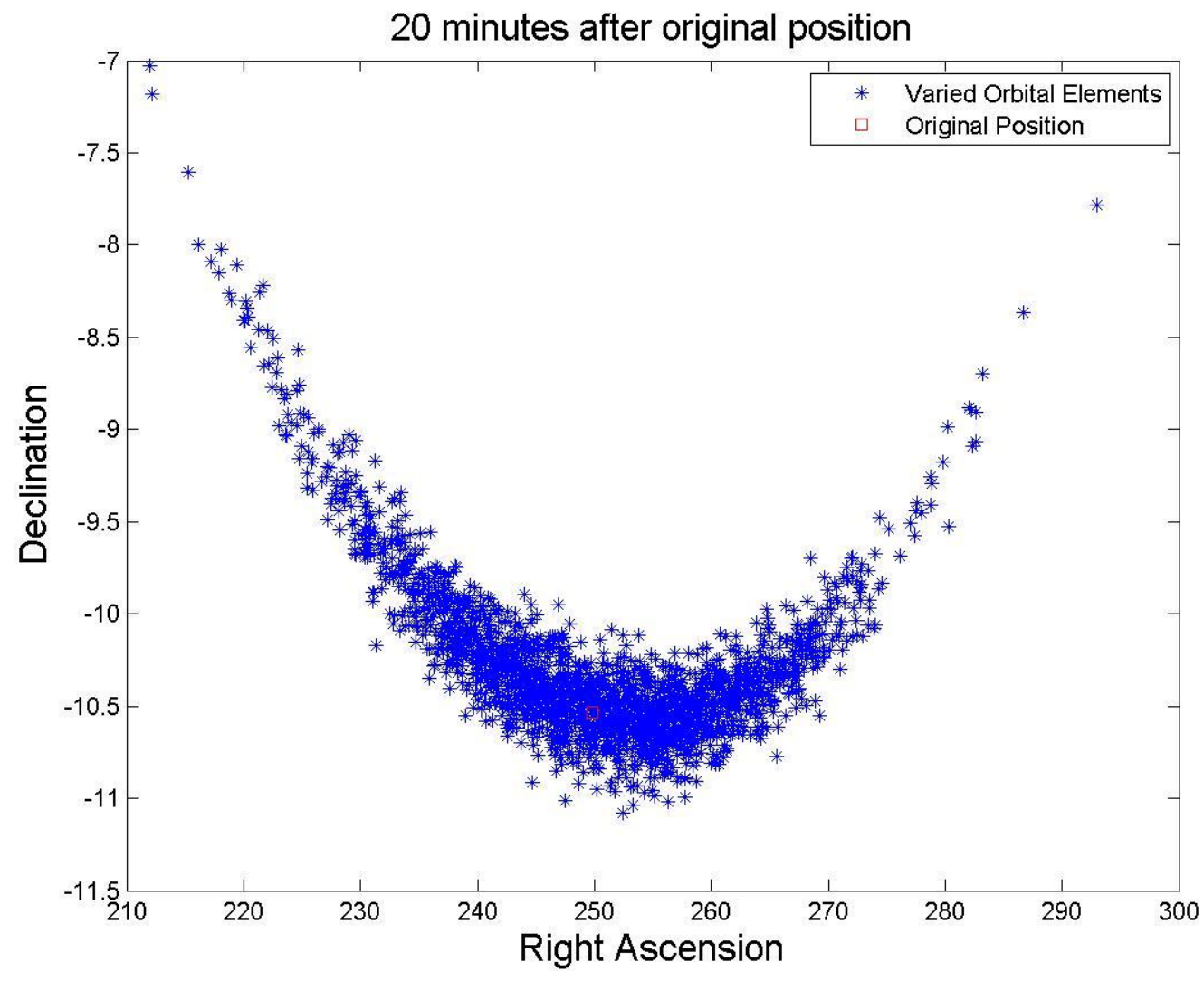



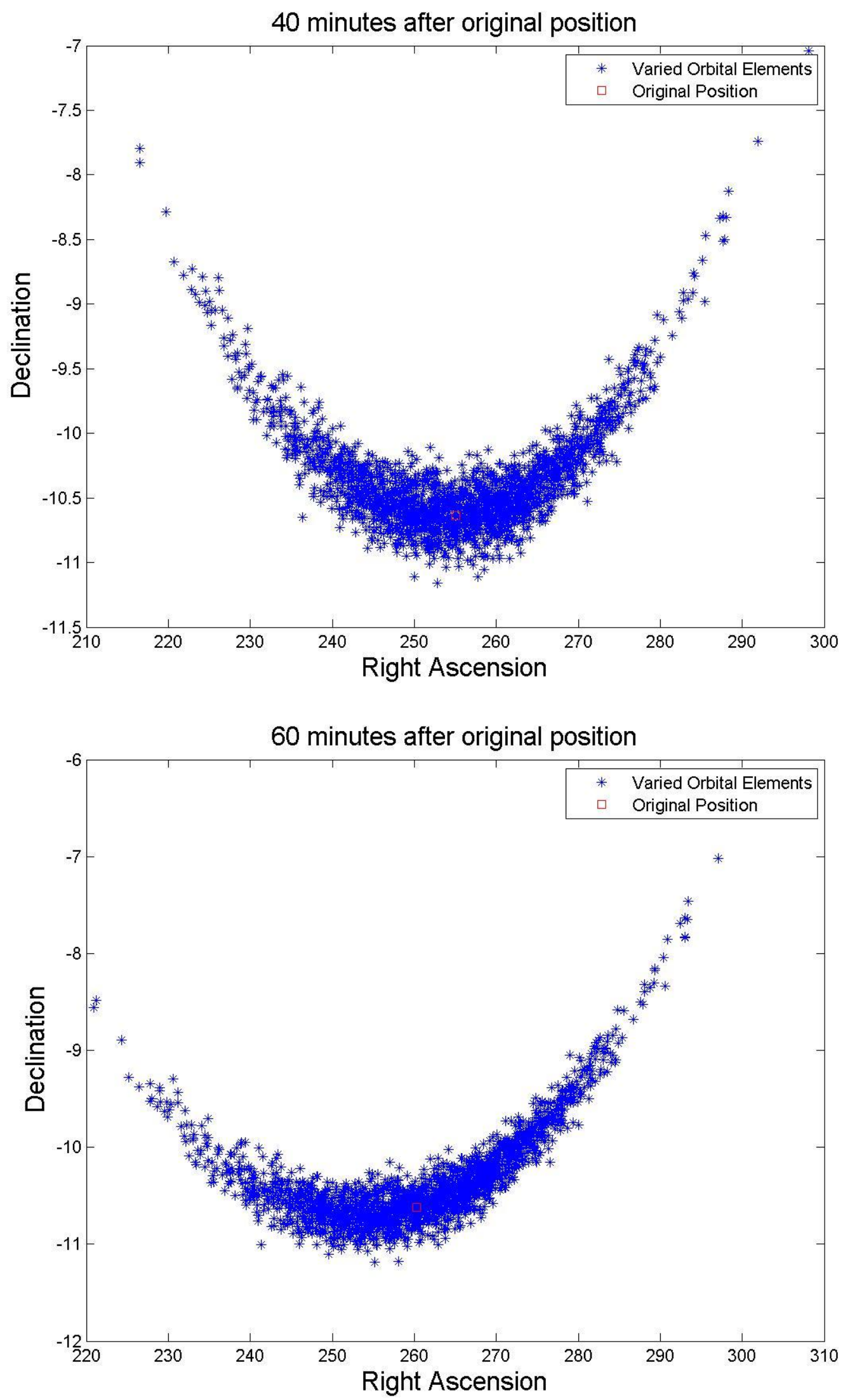


\section{Object 70303}

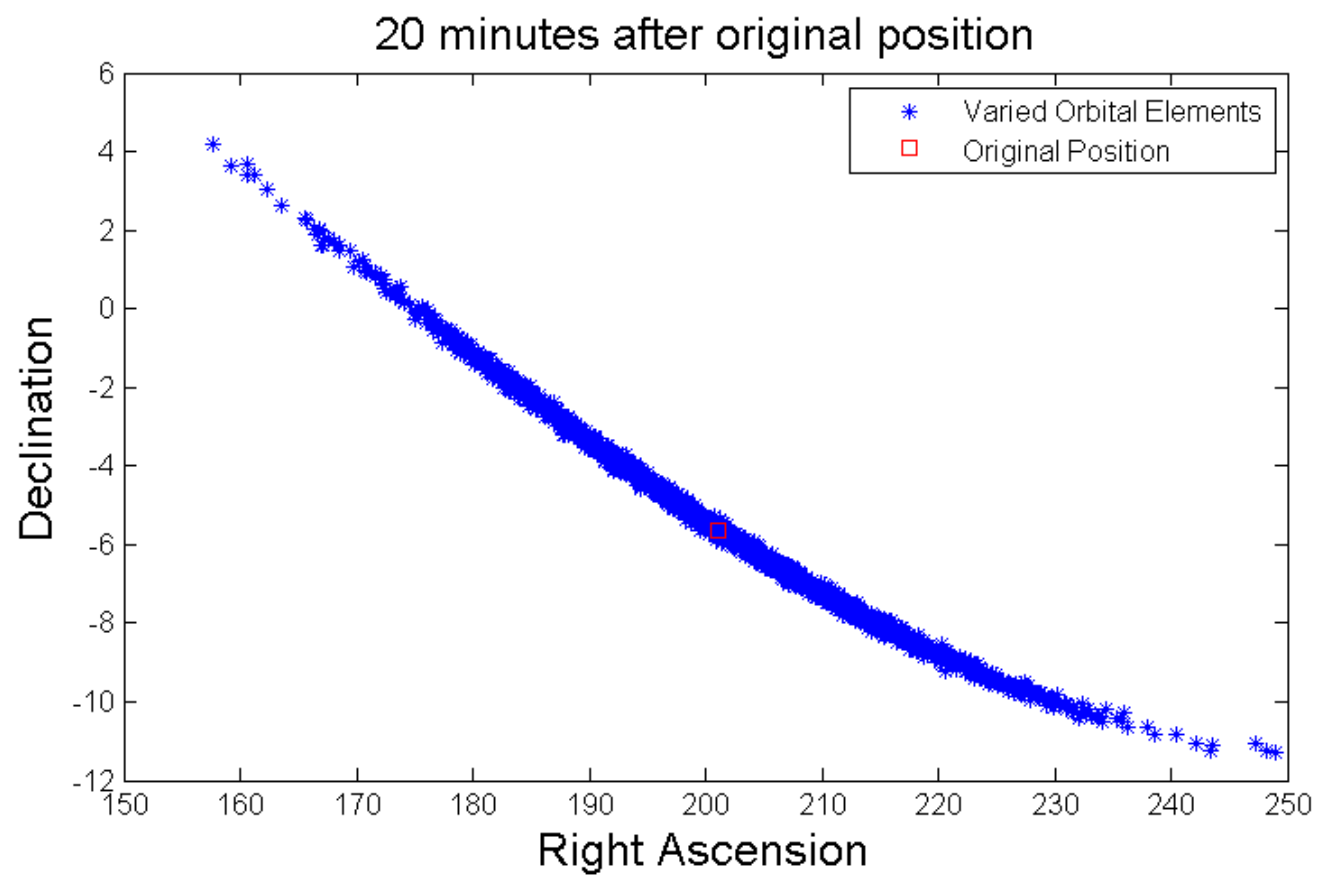



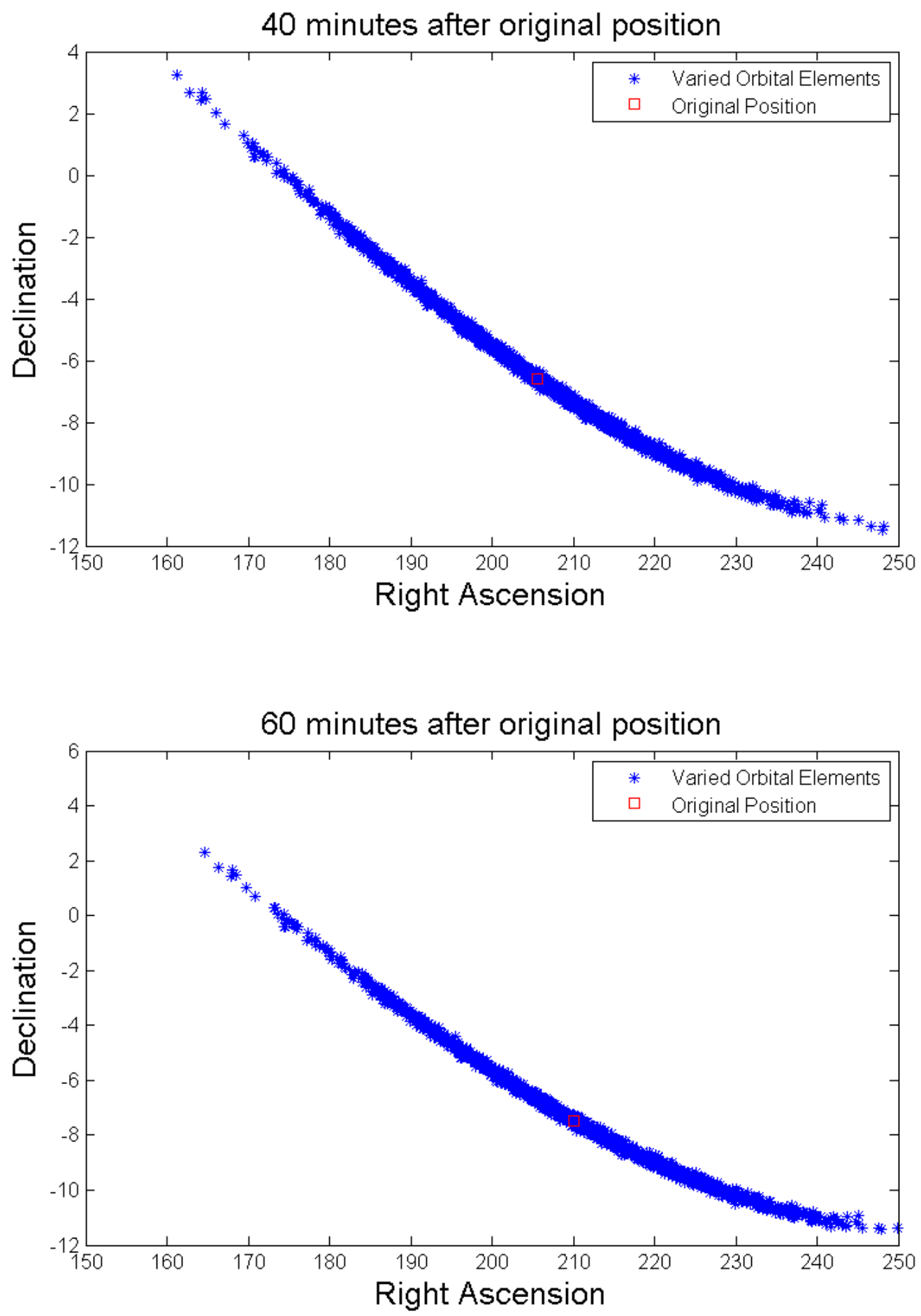


\section{Object 70304}

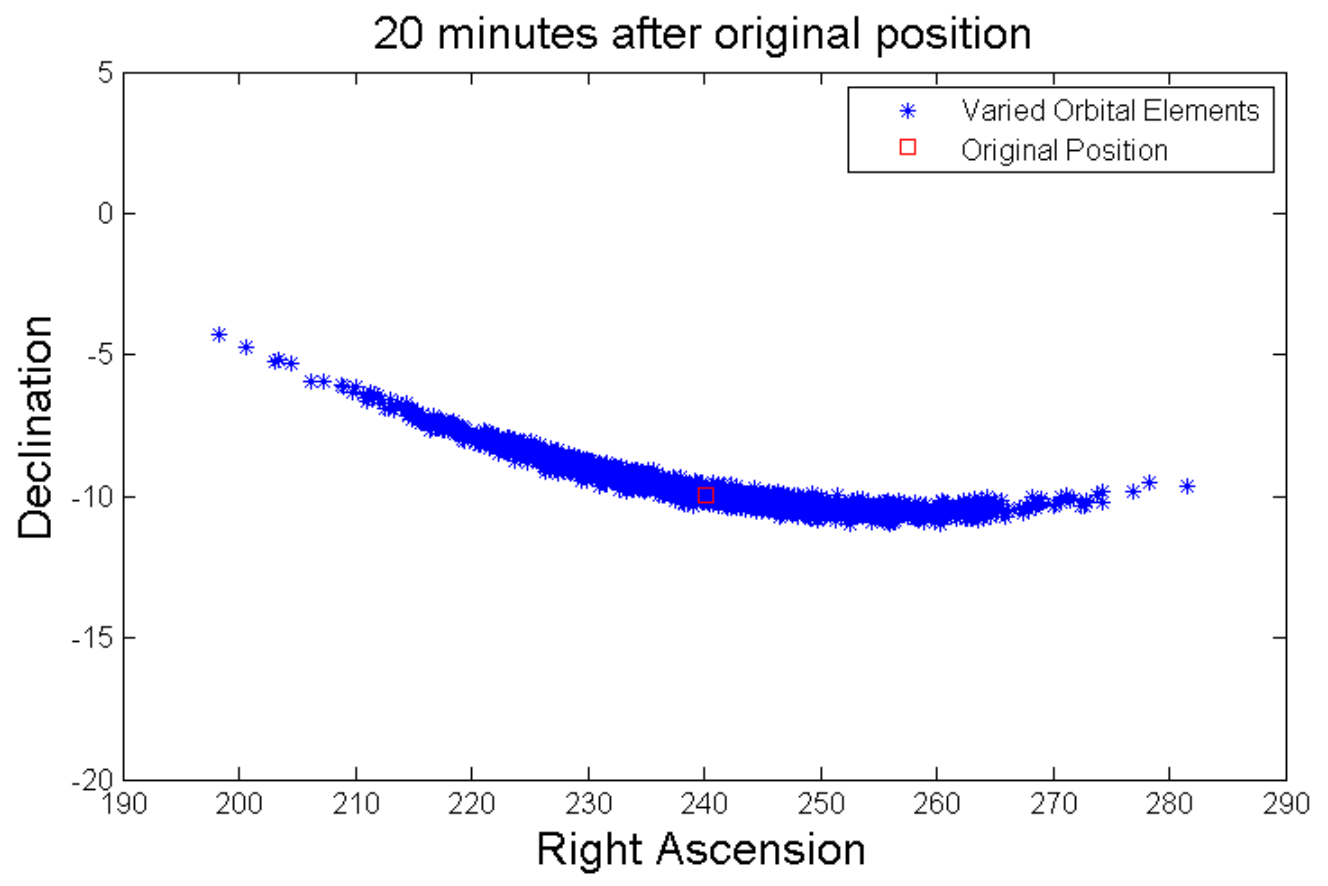



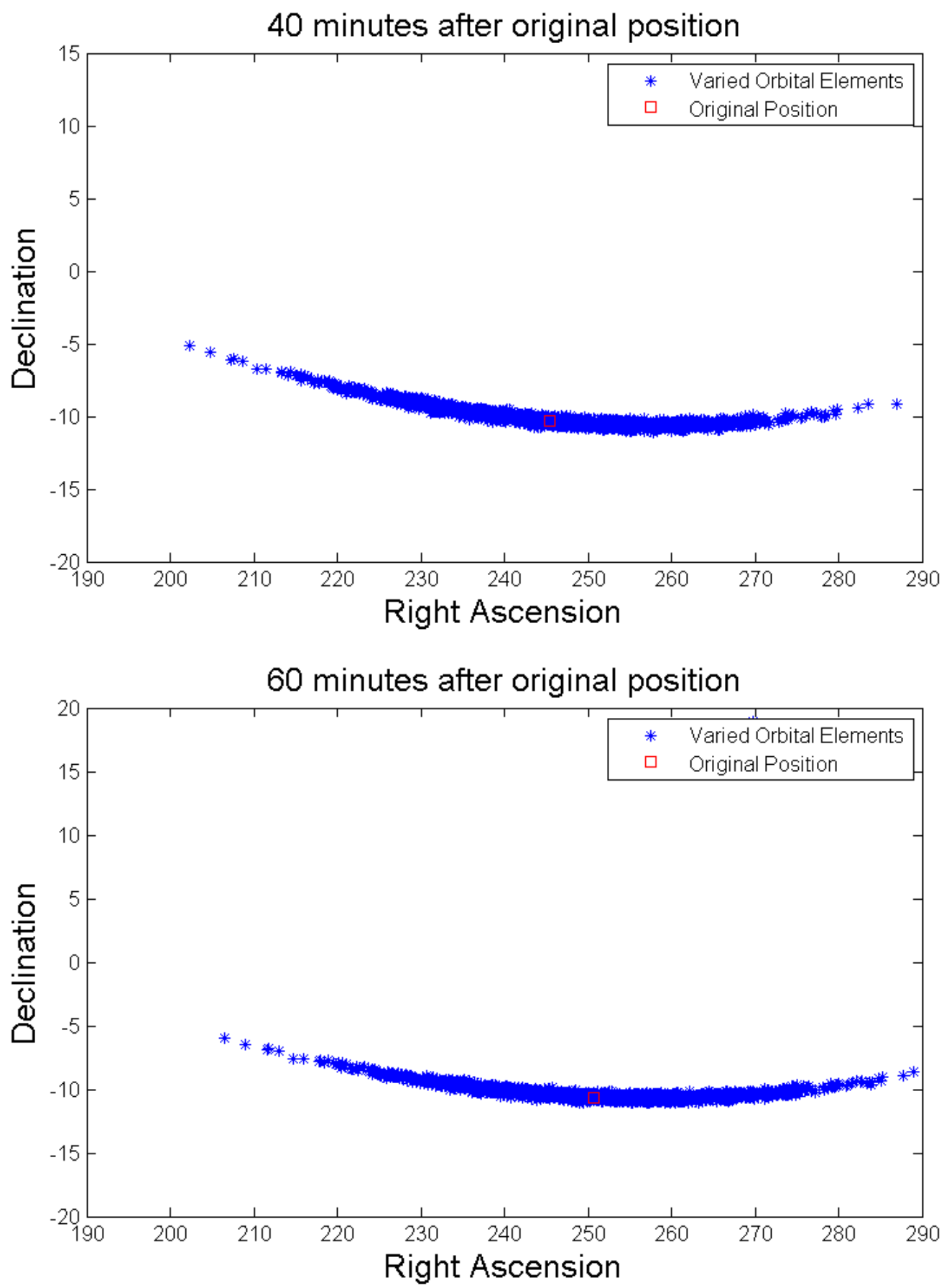


\section{Object 70305}

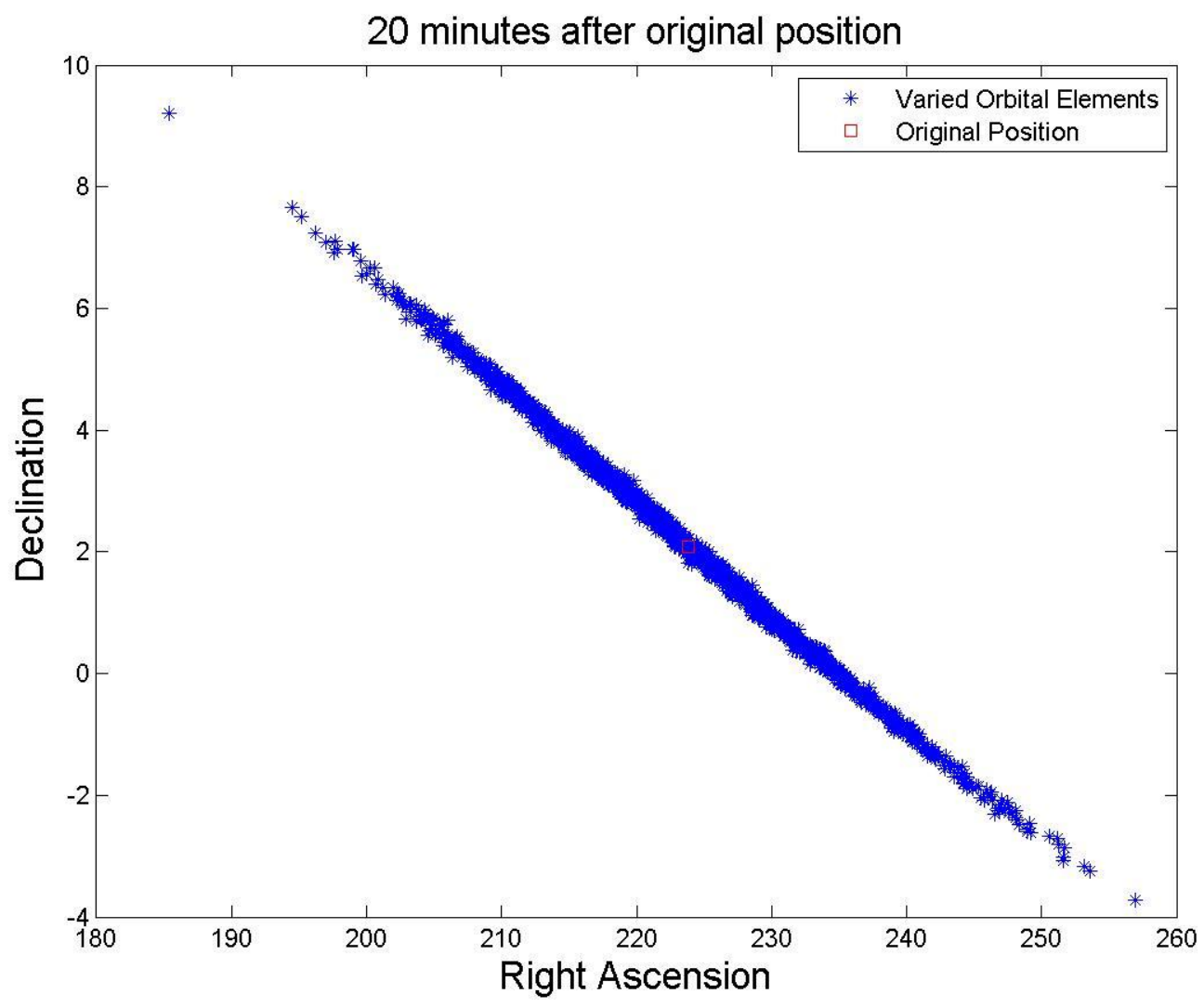



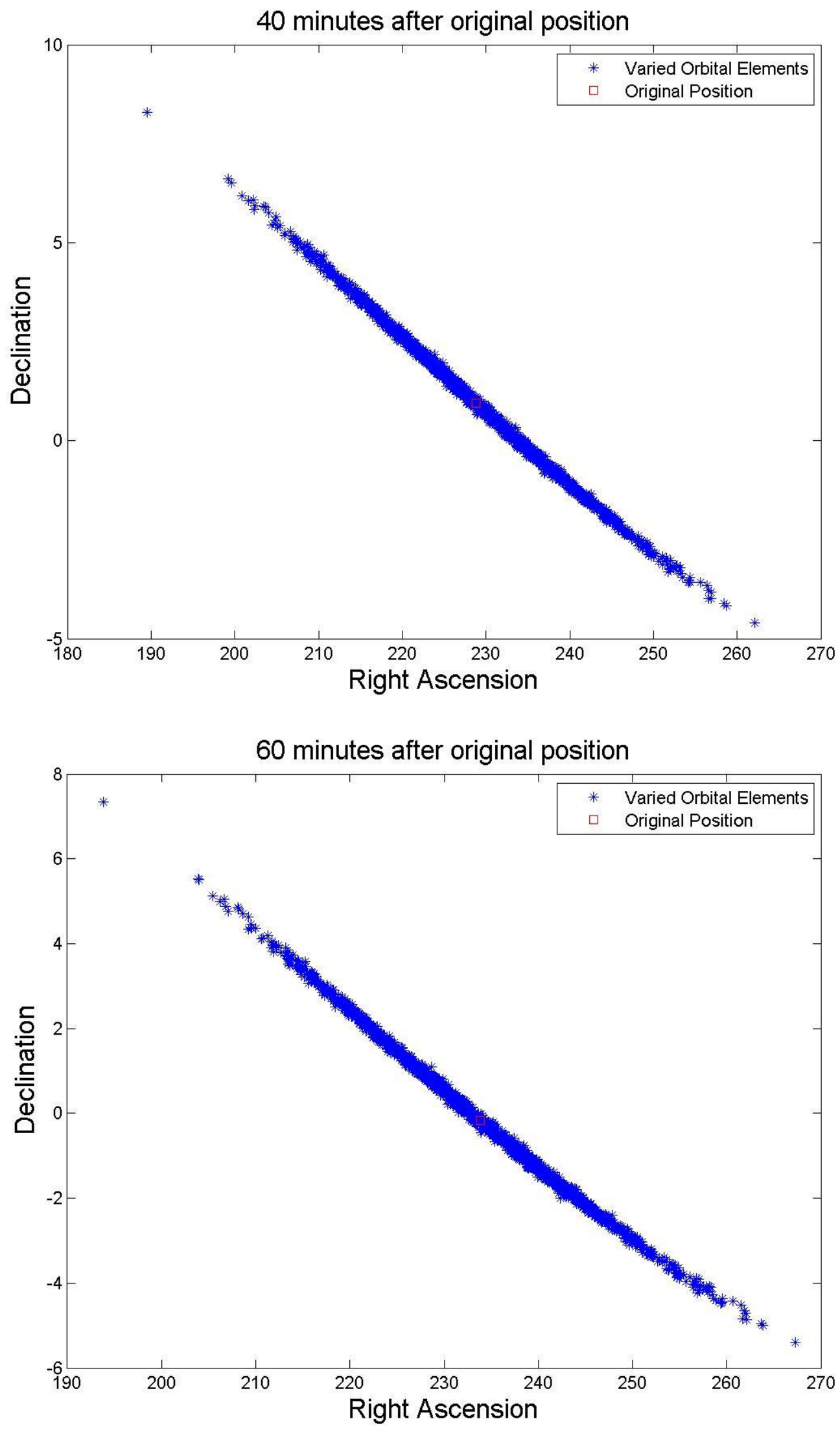


\section{Object 70306}

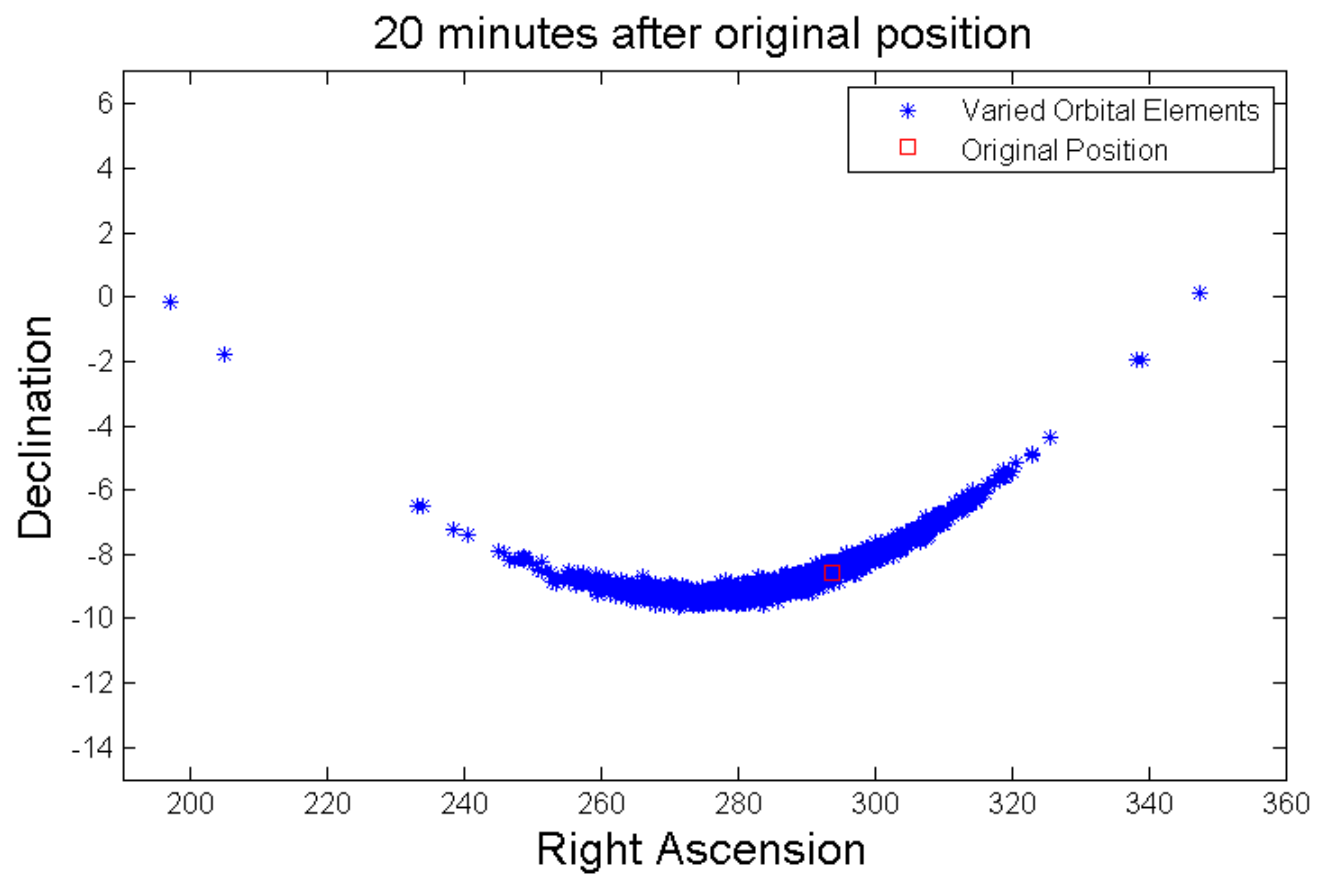



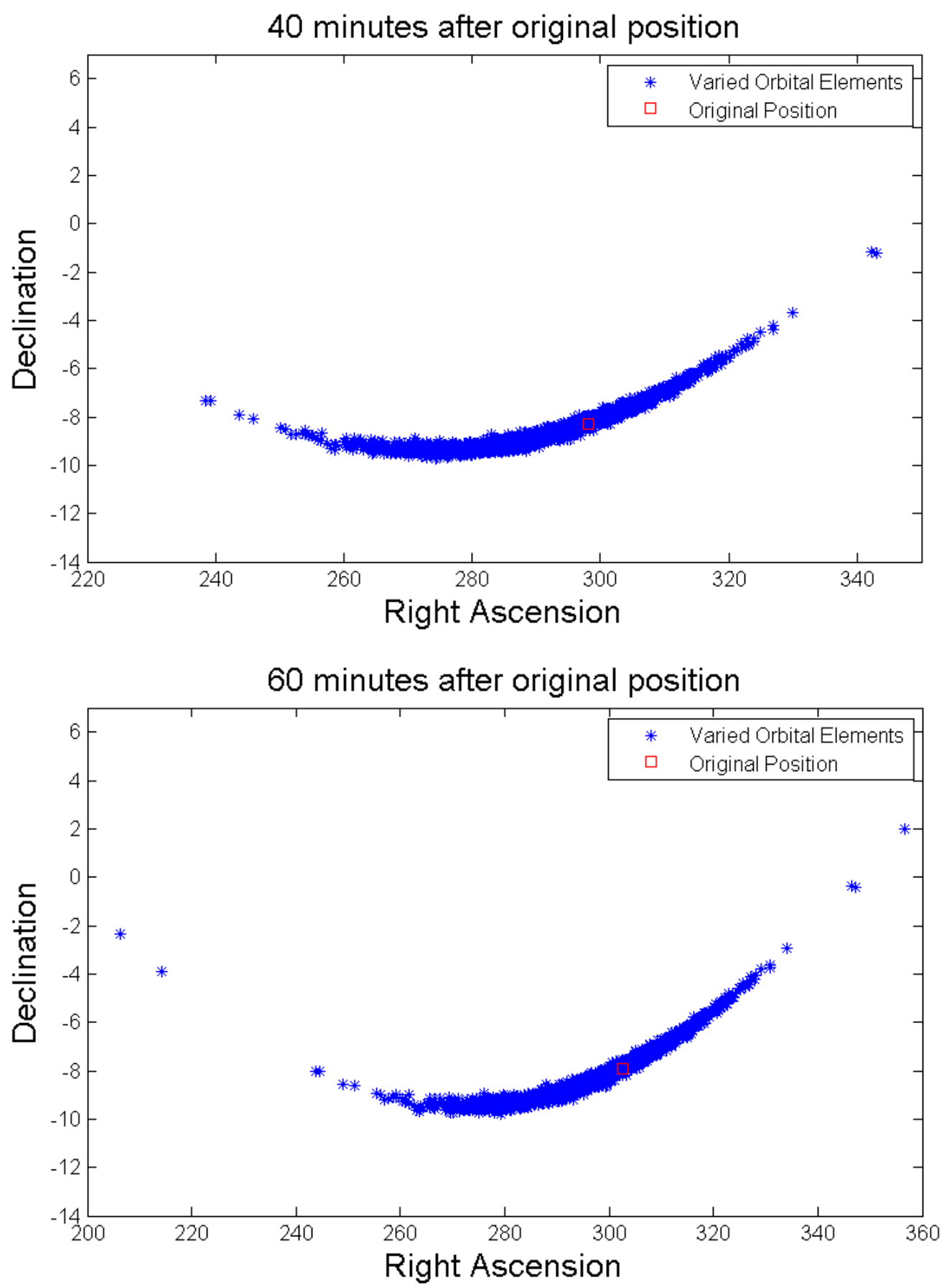


\section{Object 70307}

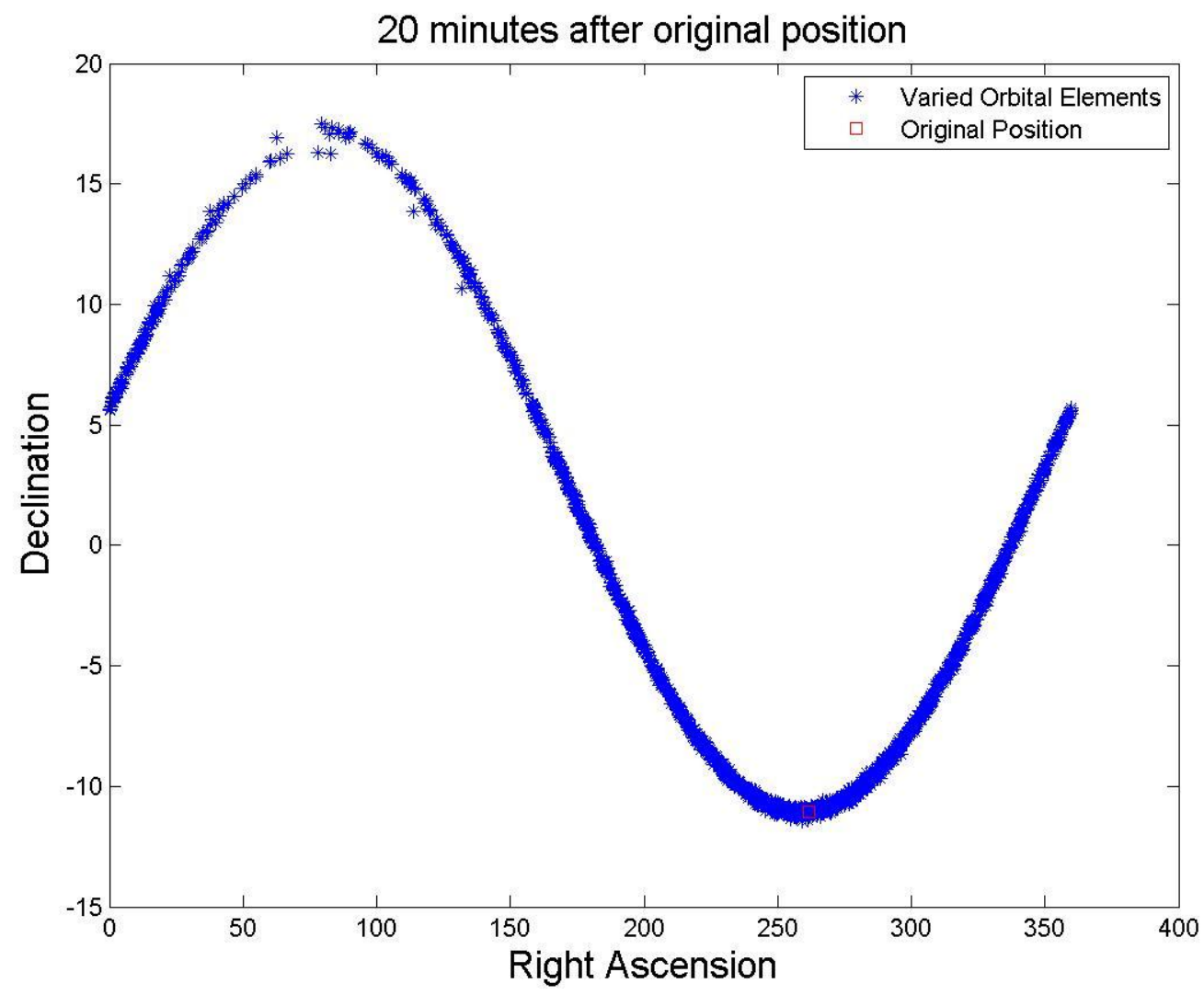



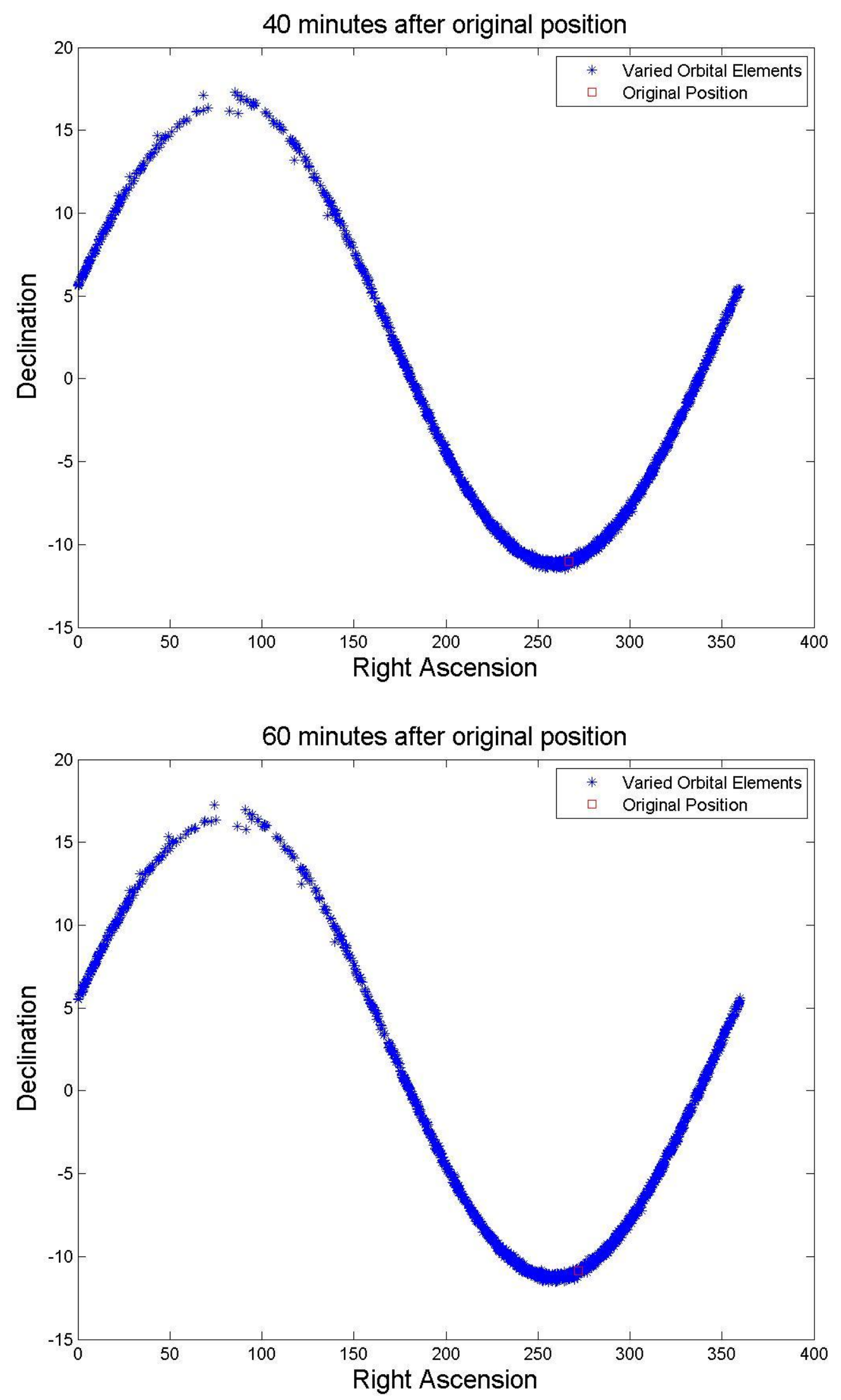


\section{Object 70308}

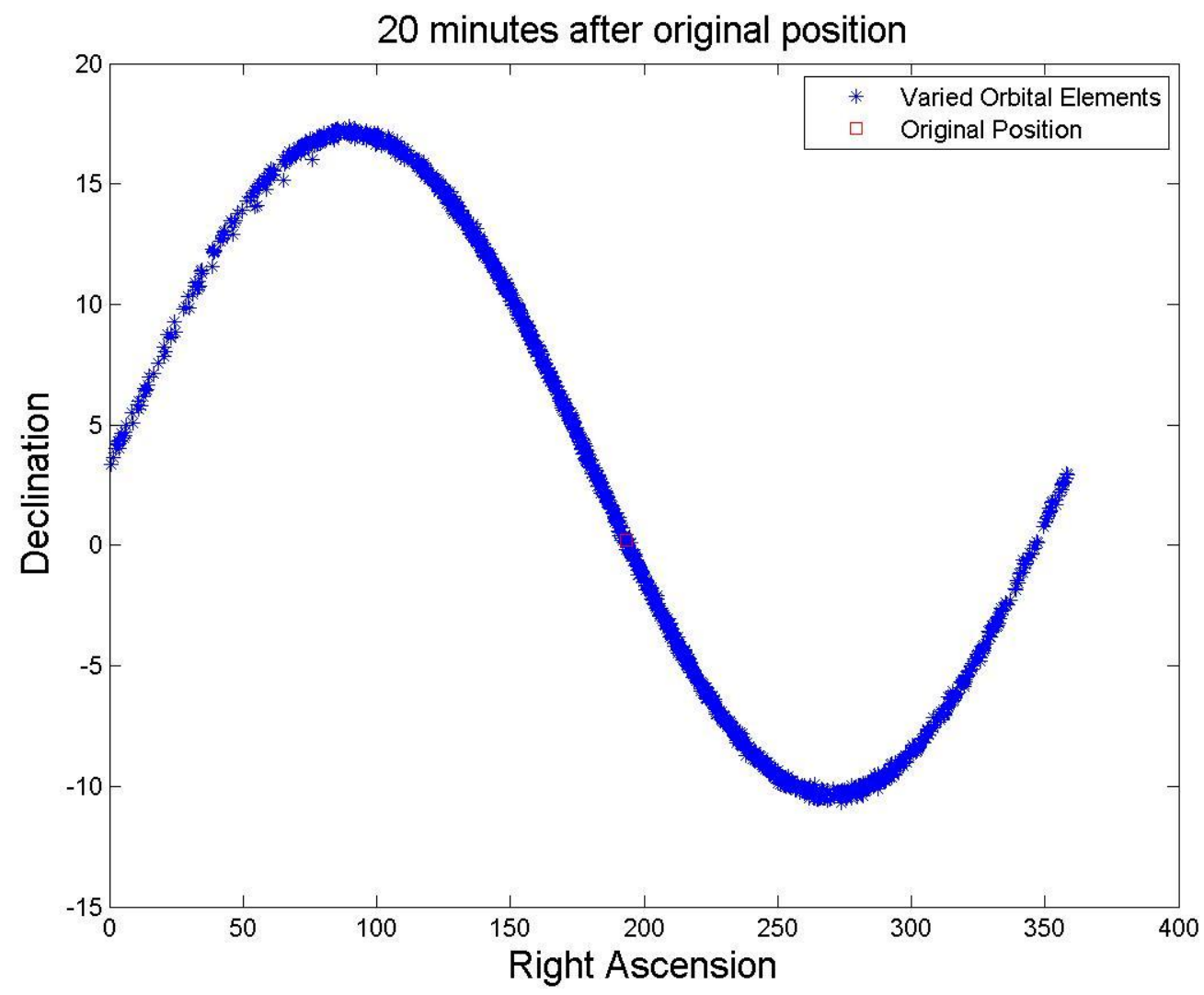



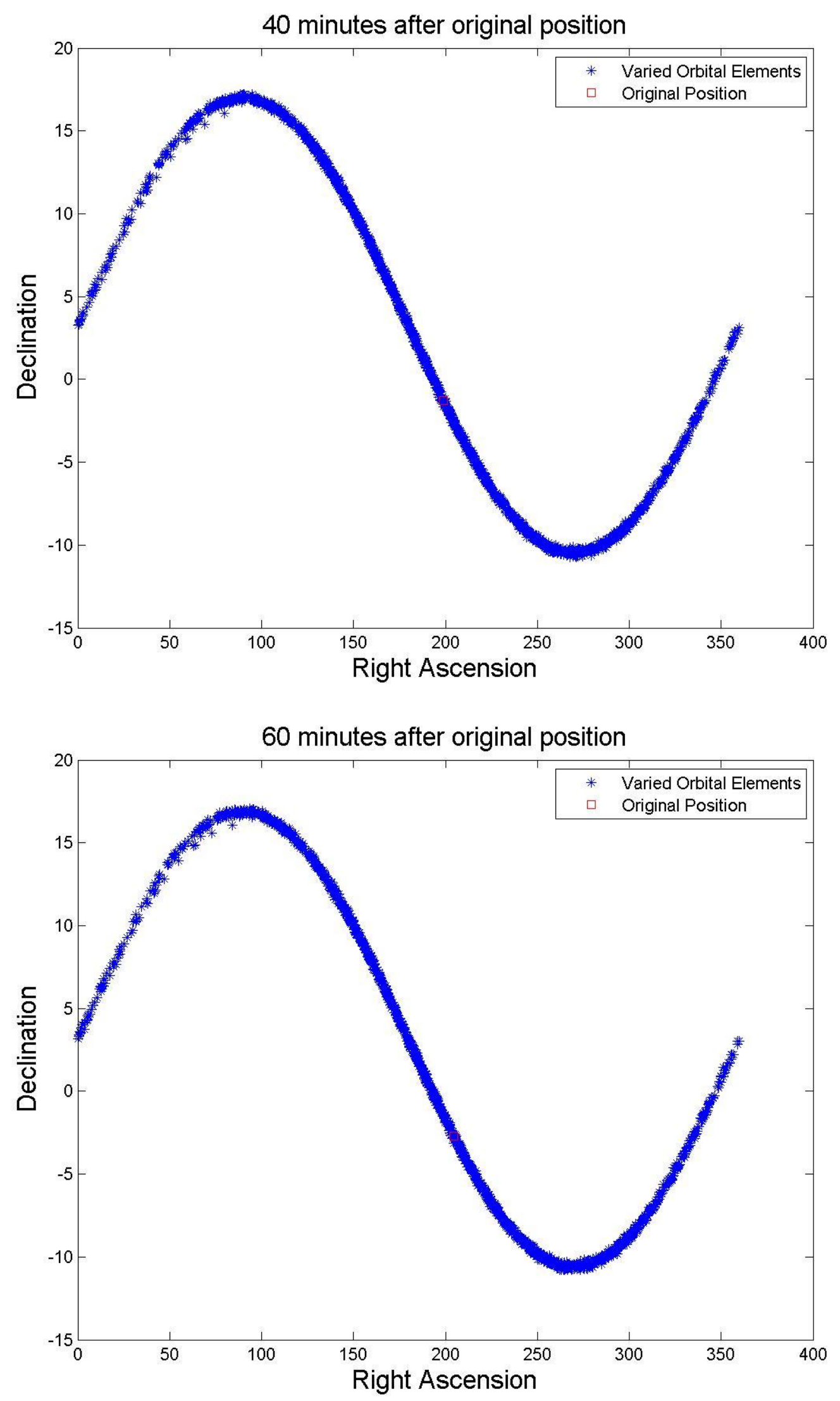


\section{Object 70309}

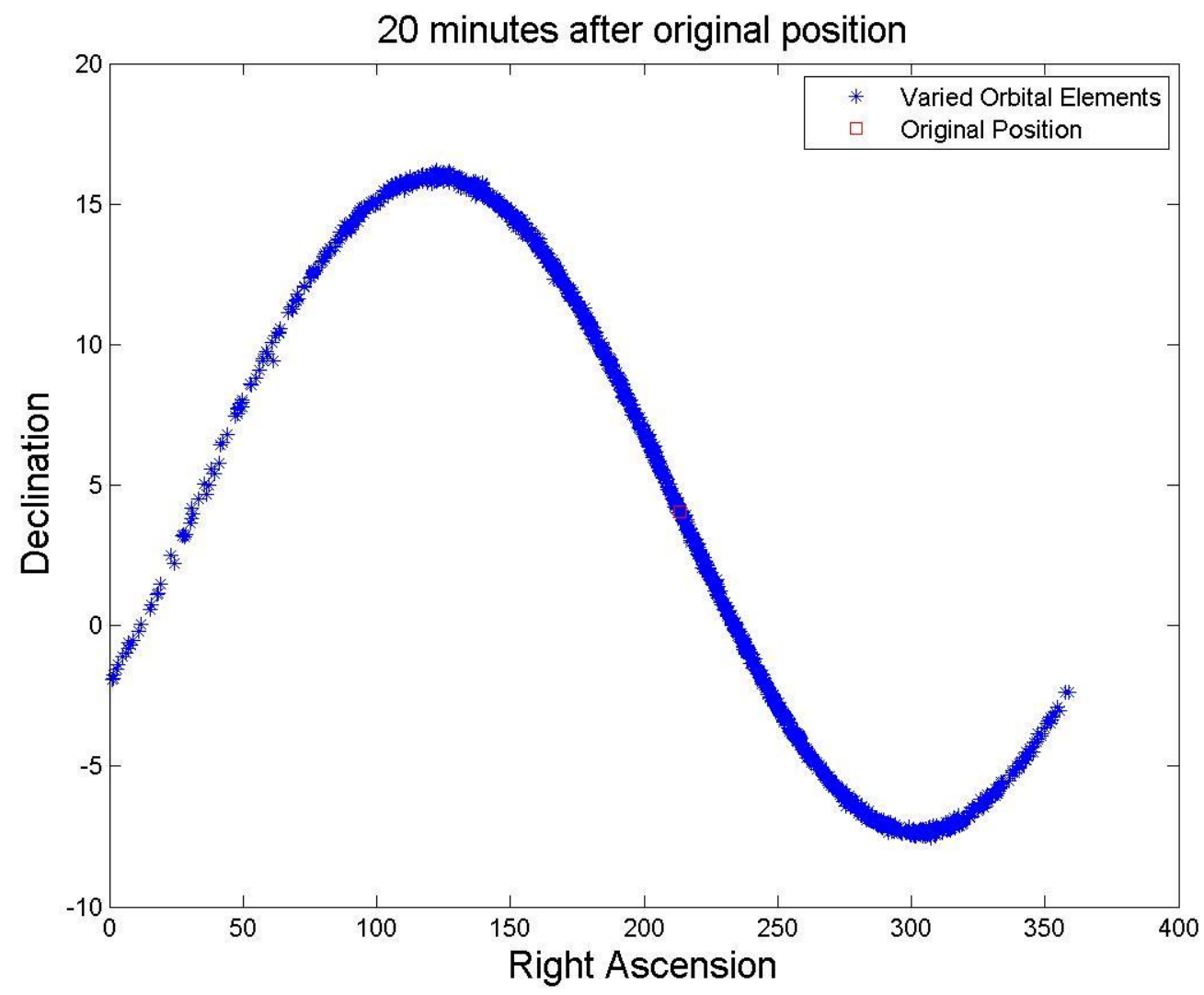



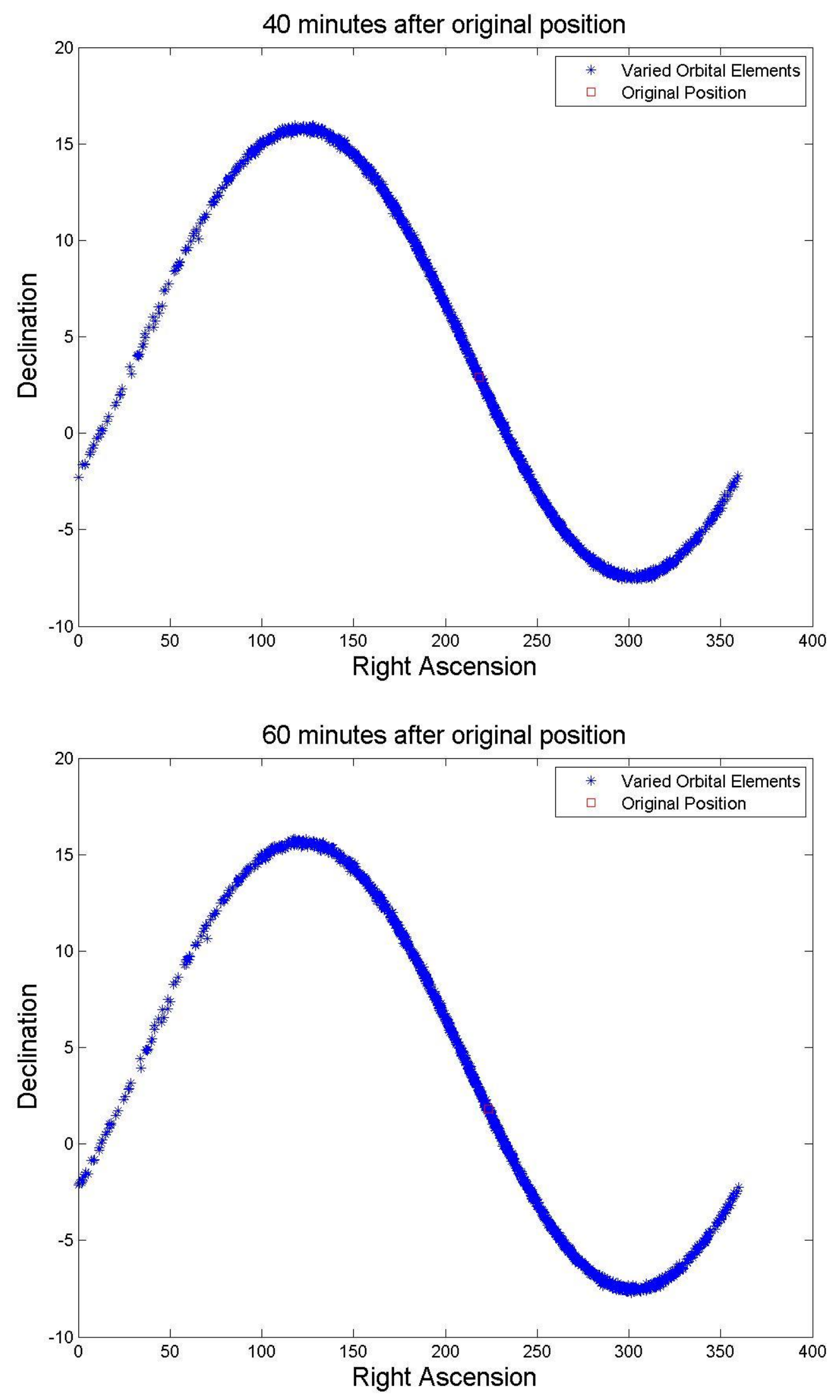


\section{Object 70310}

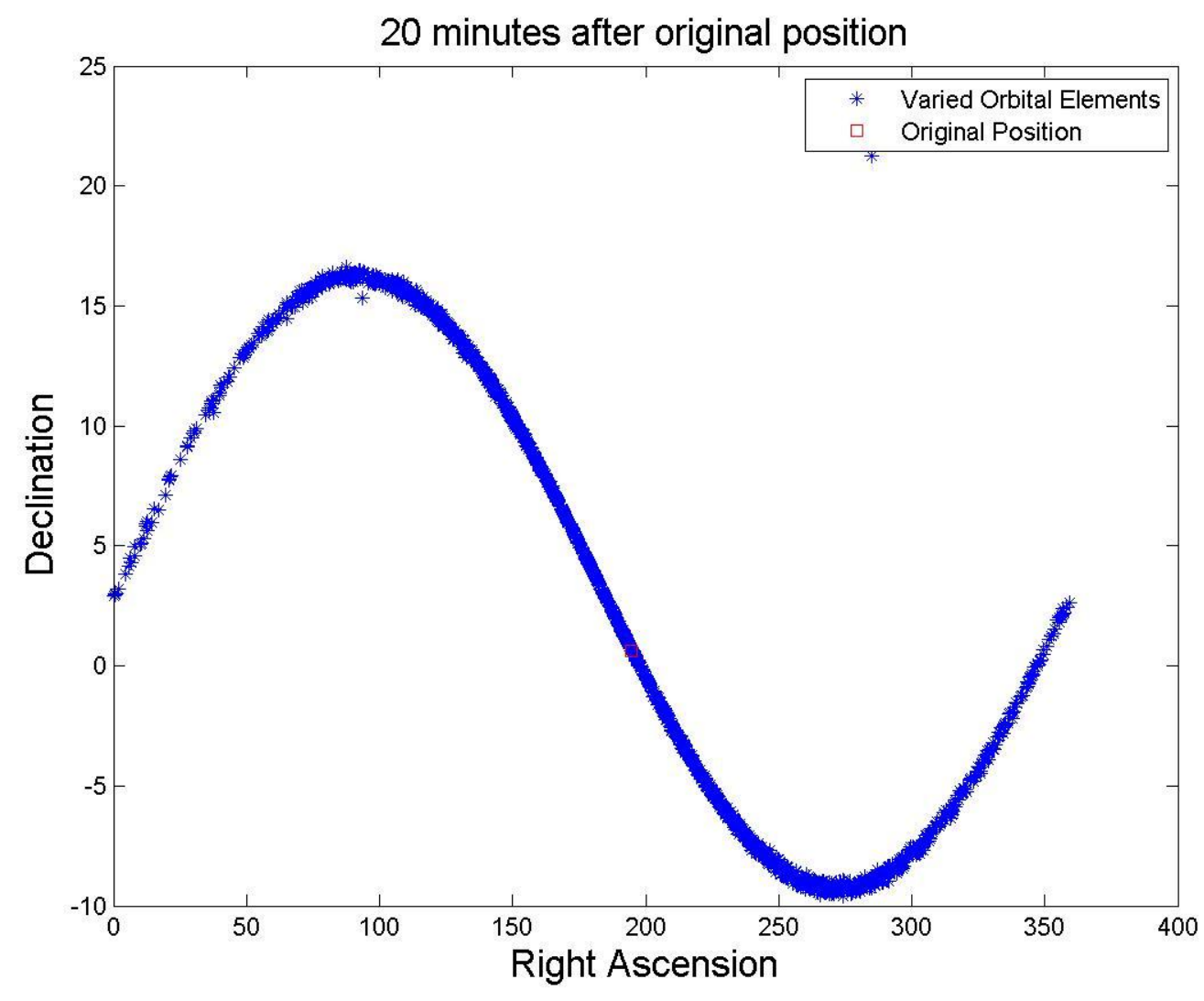



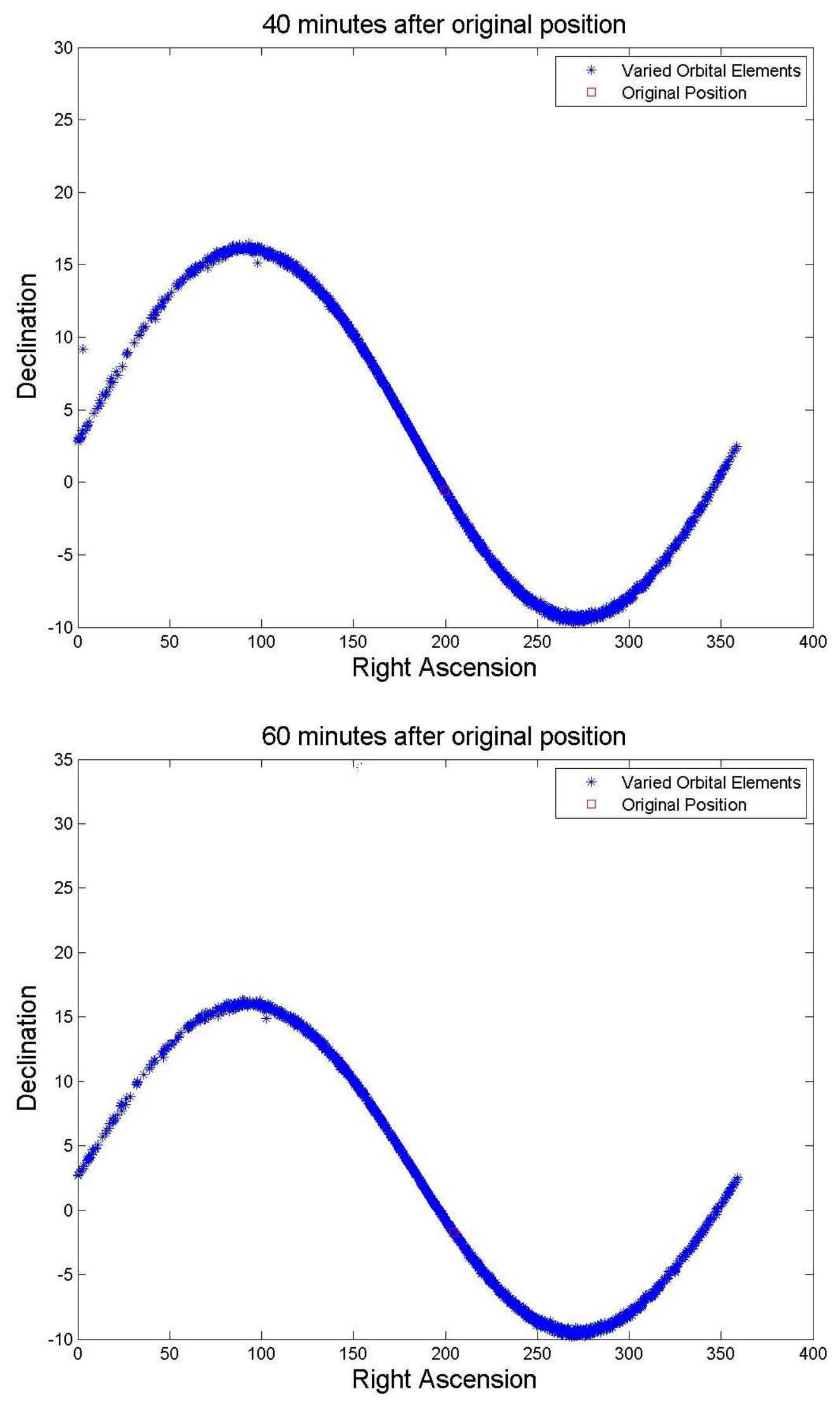


\section{Object 70311}

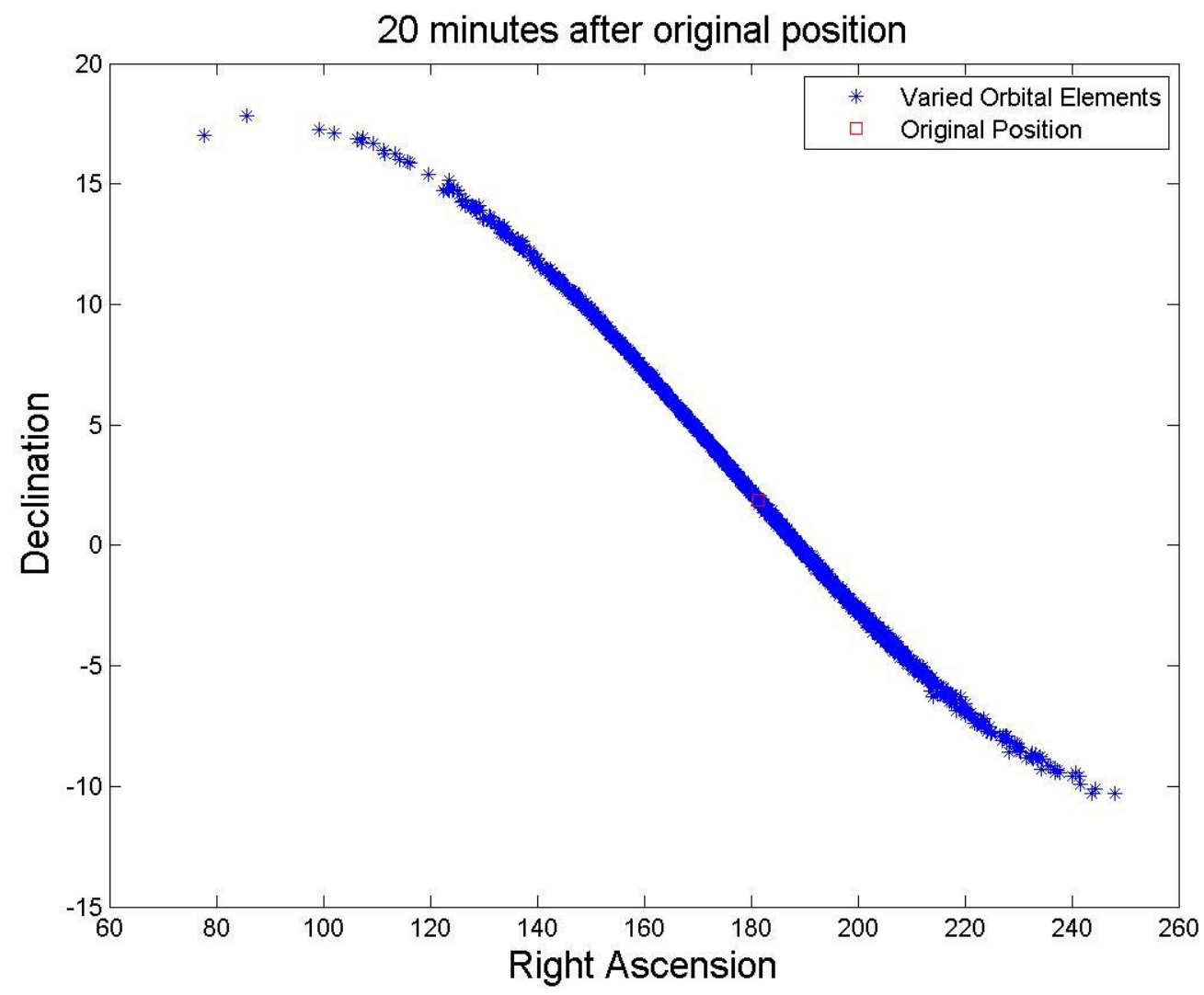



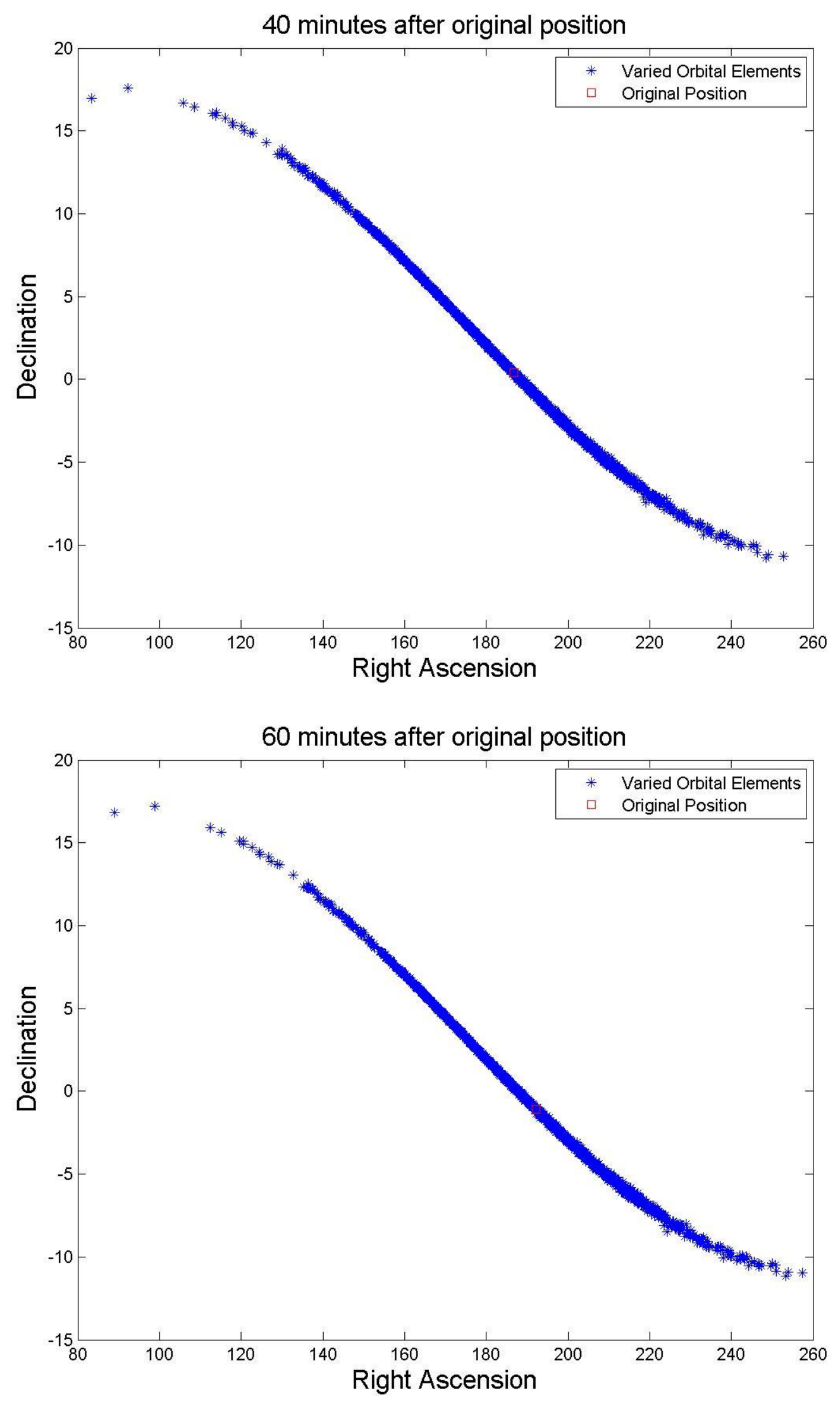


\section{Object 70312}

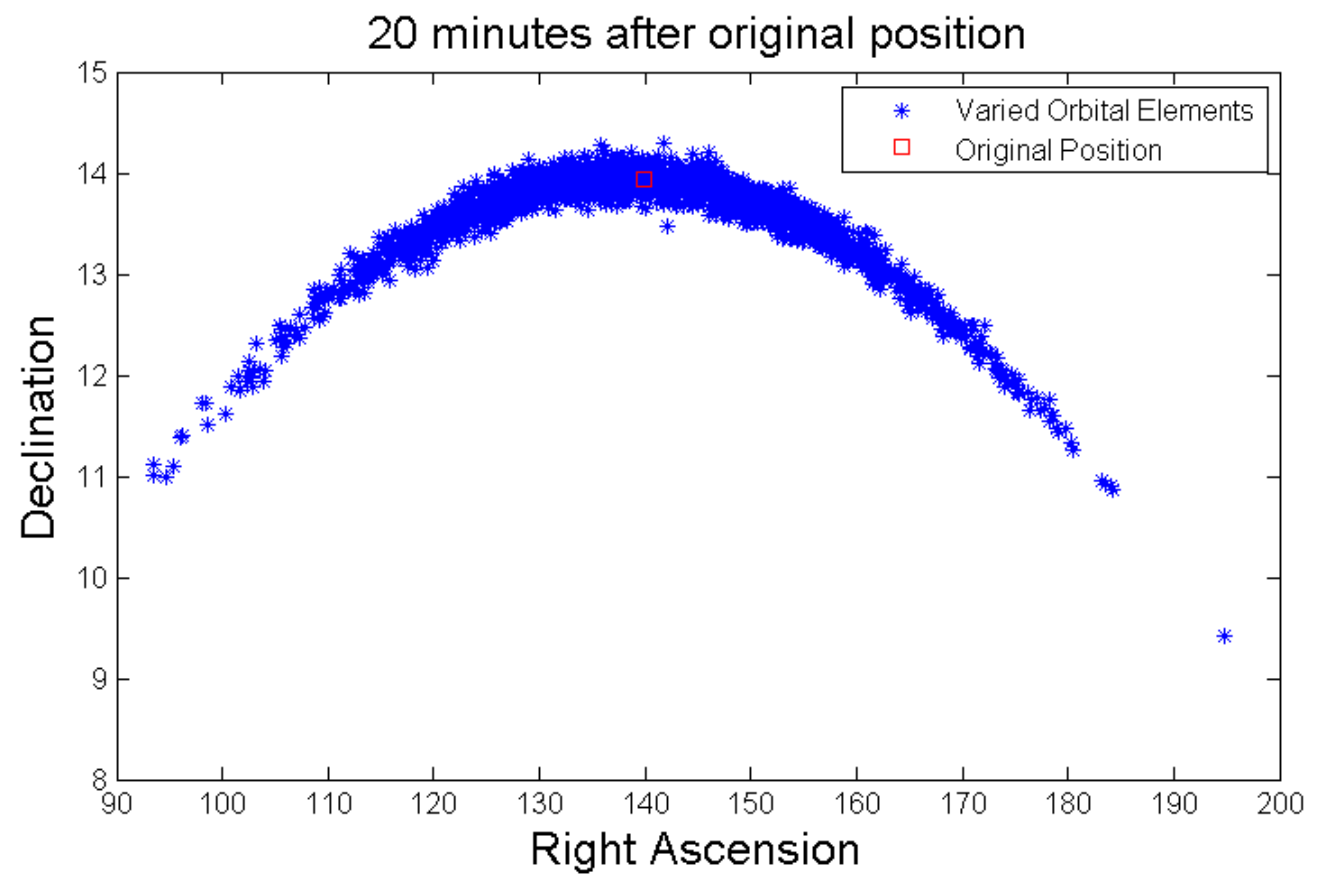



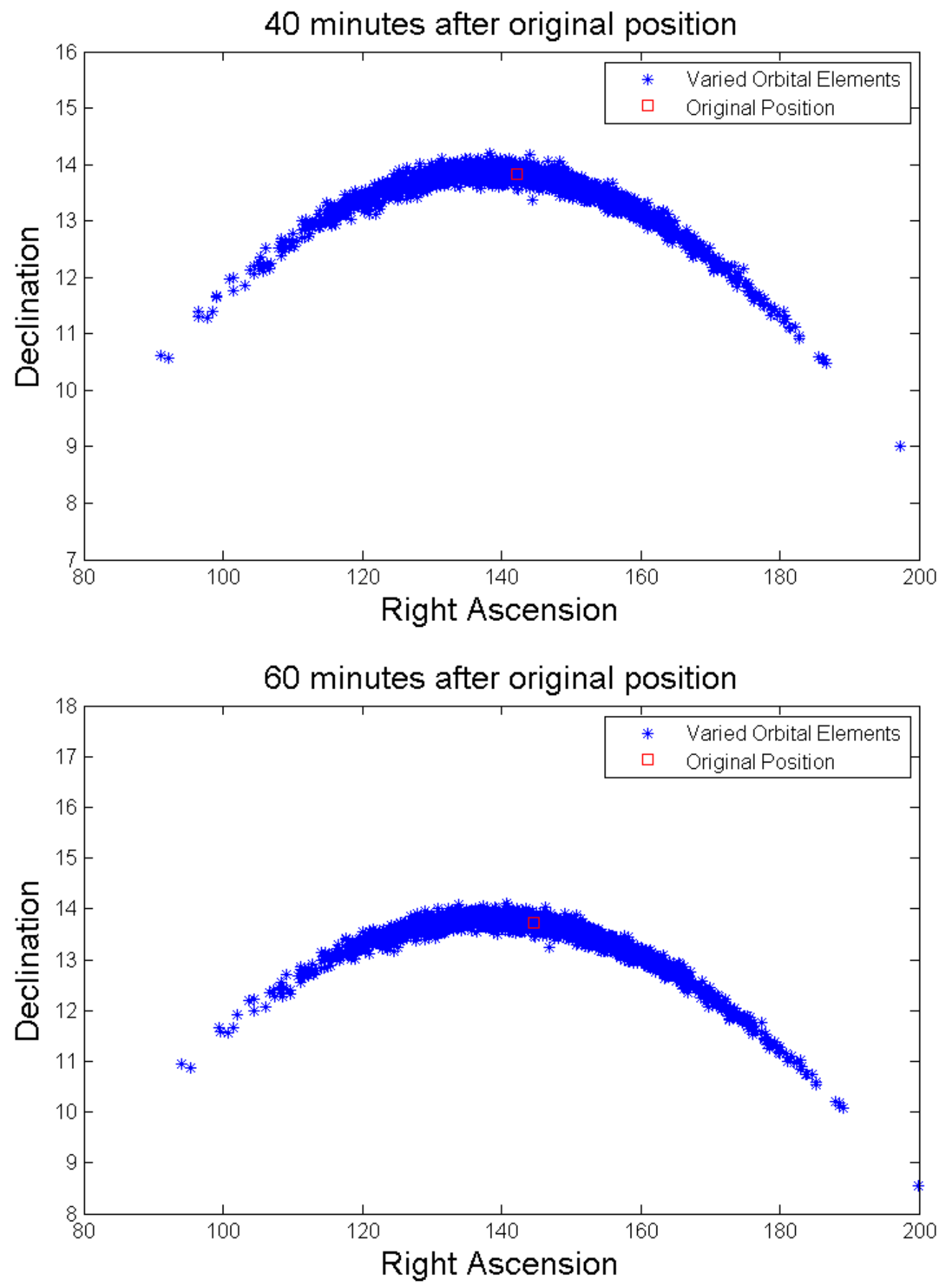


\section{Object 70313}

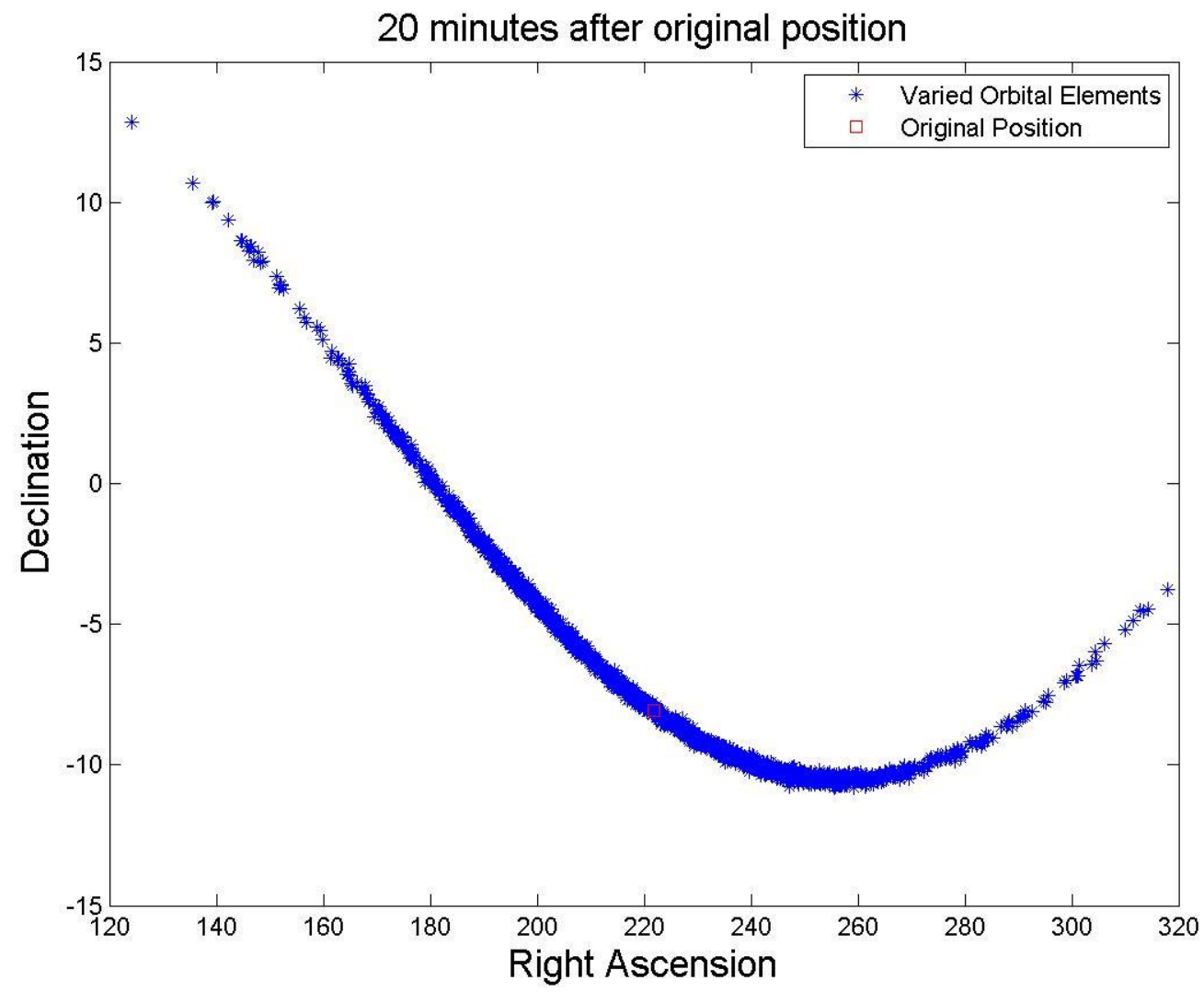



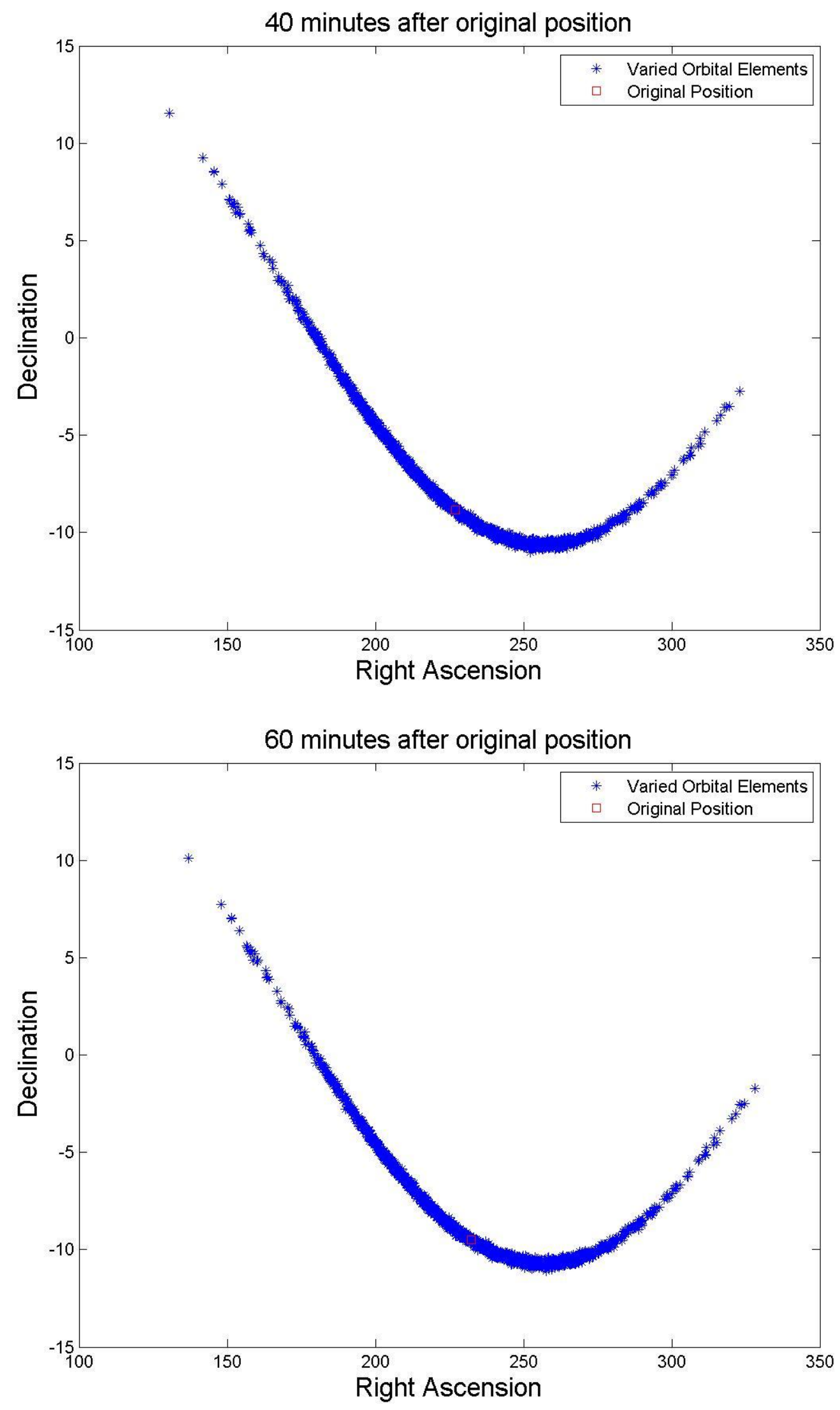


\section{Object 70315}

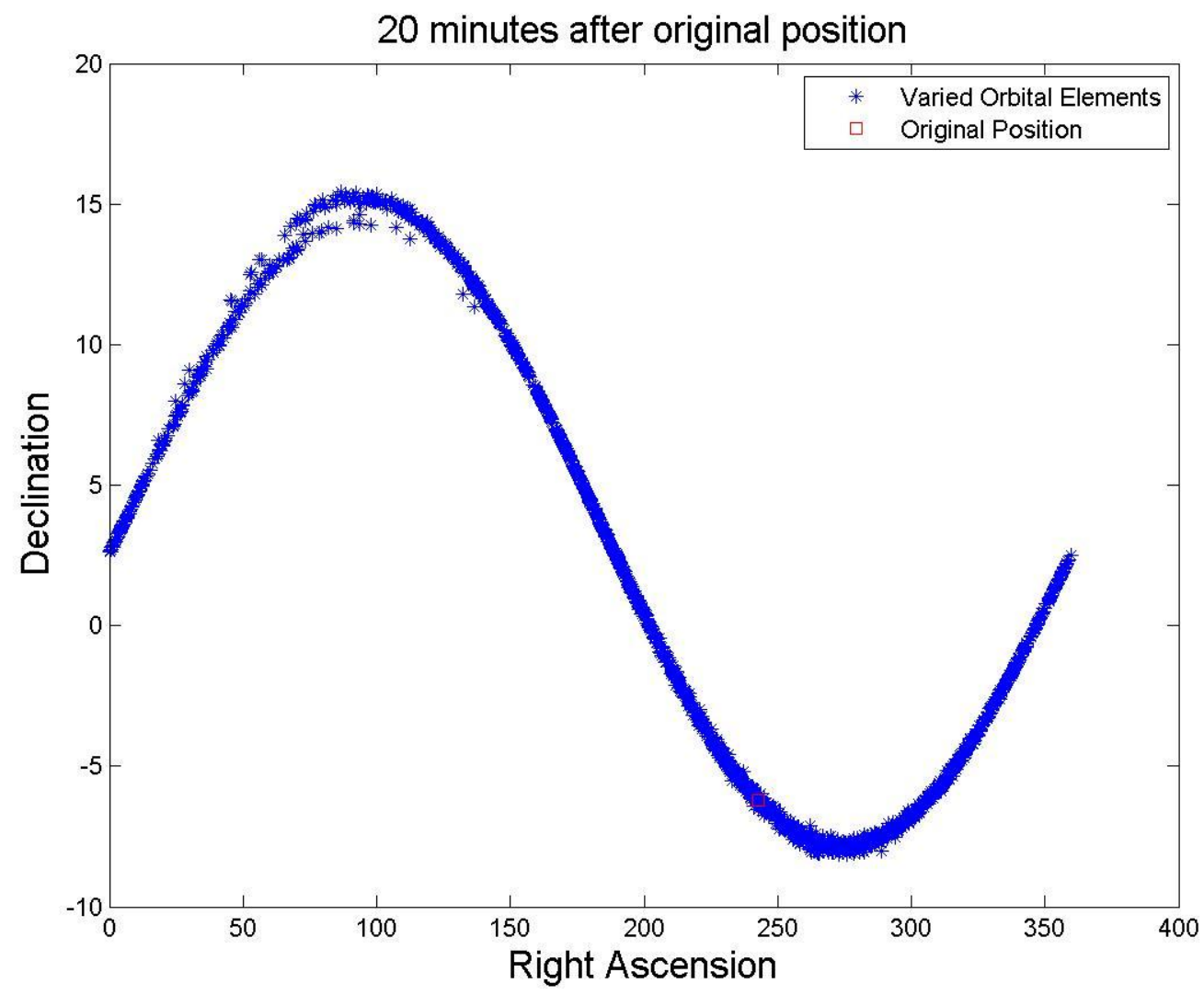



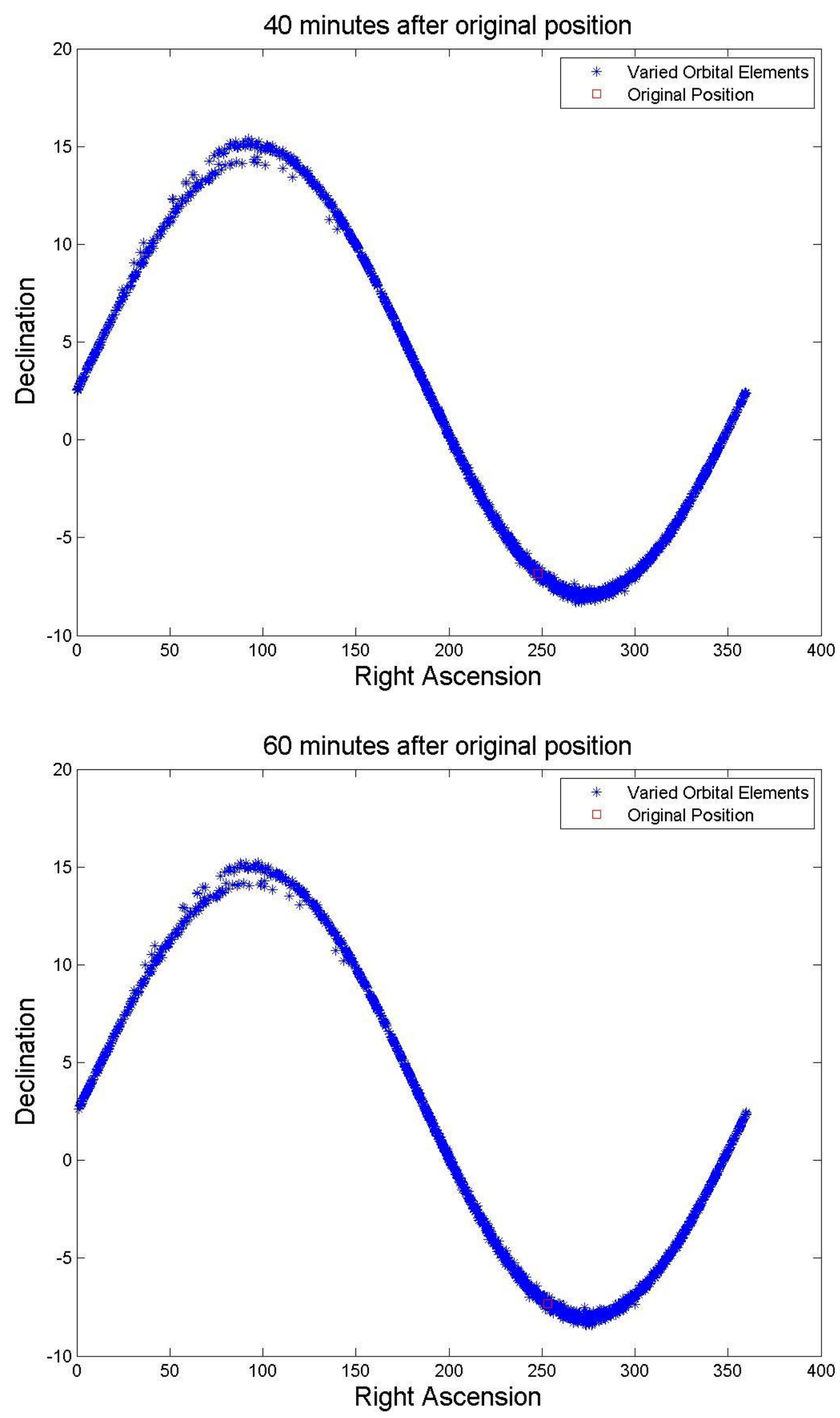


\section{Object 70316}

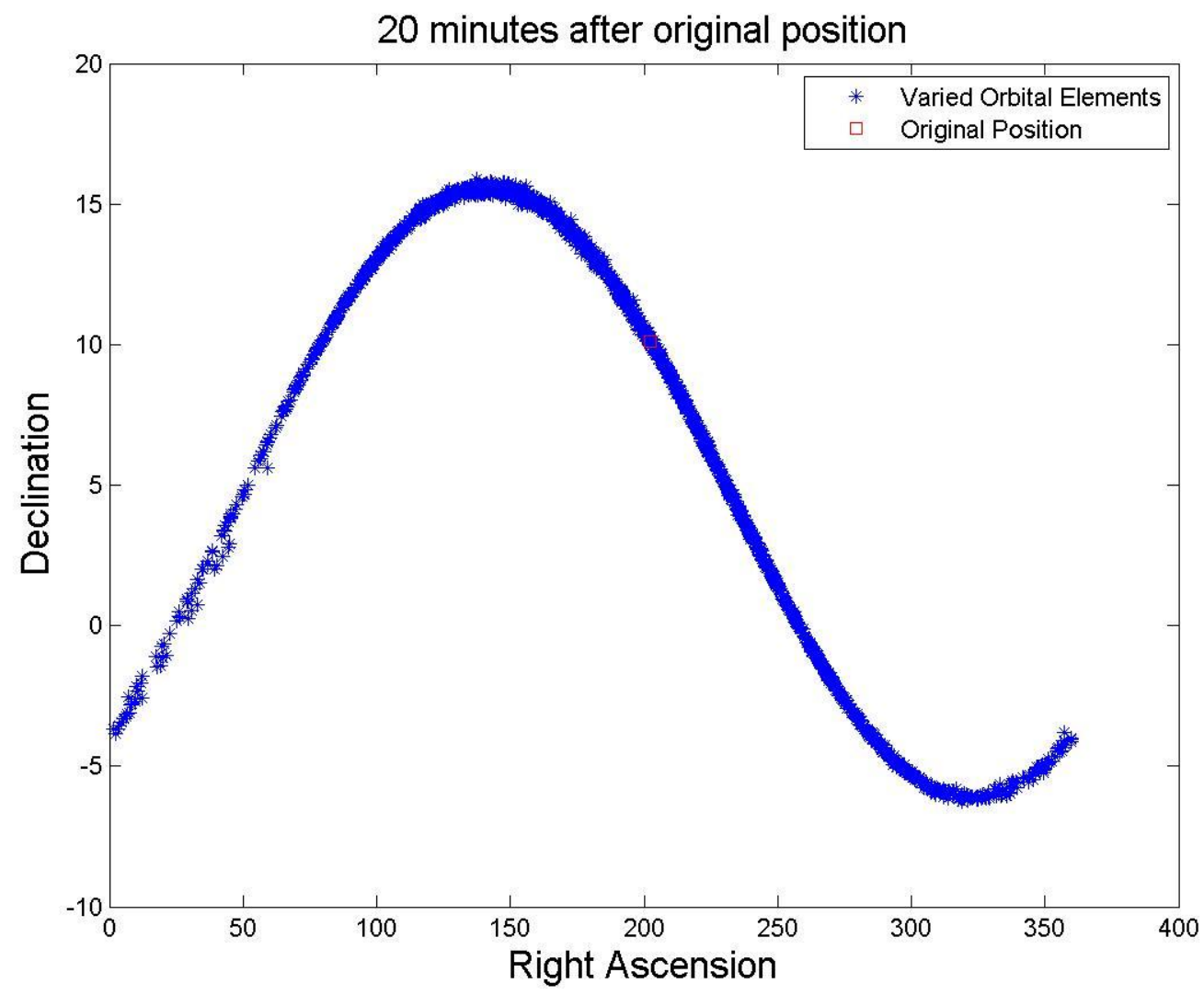



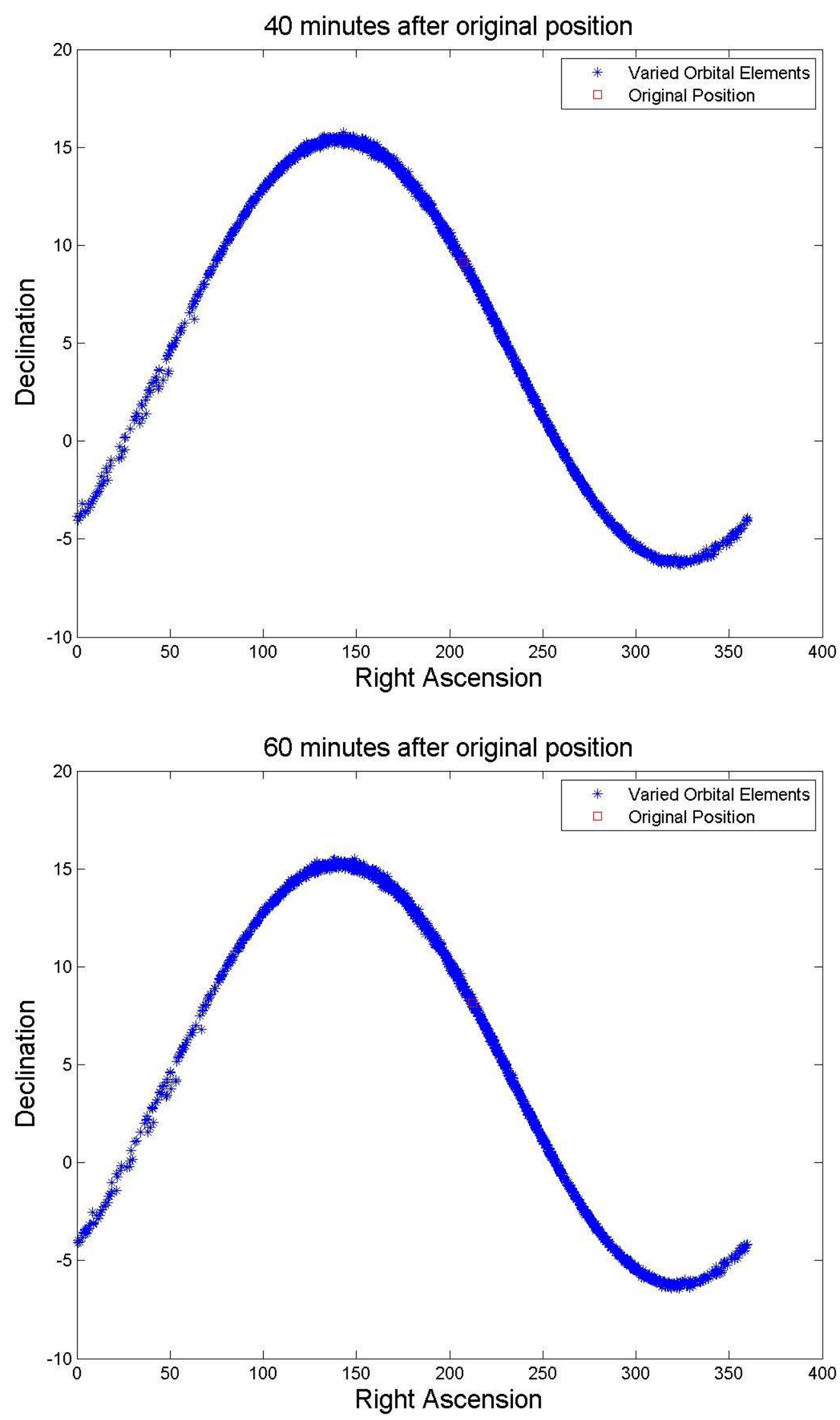


\section{Object 70317}

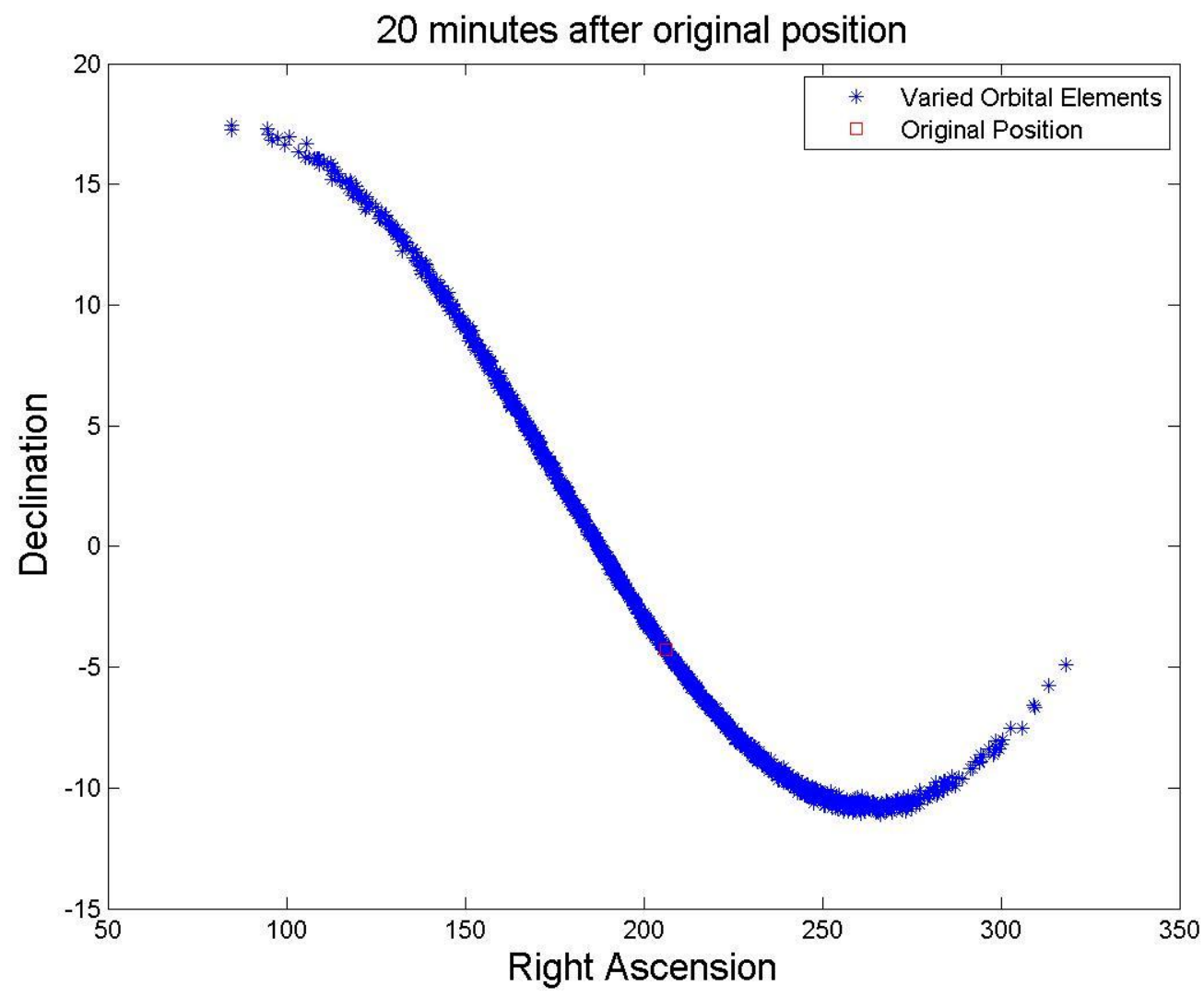



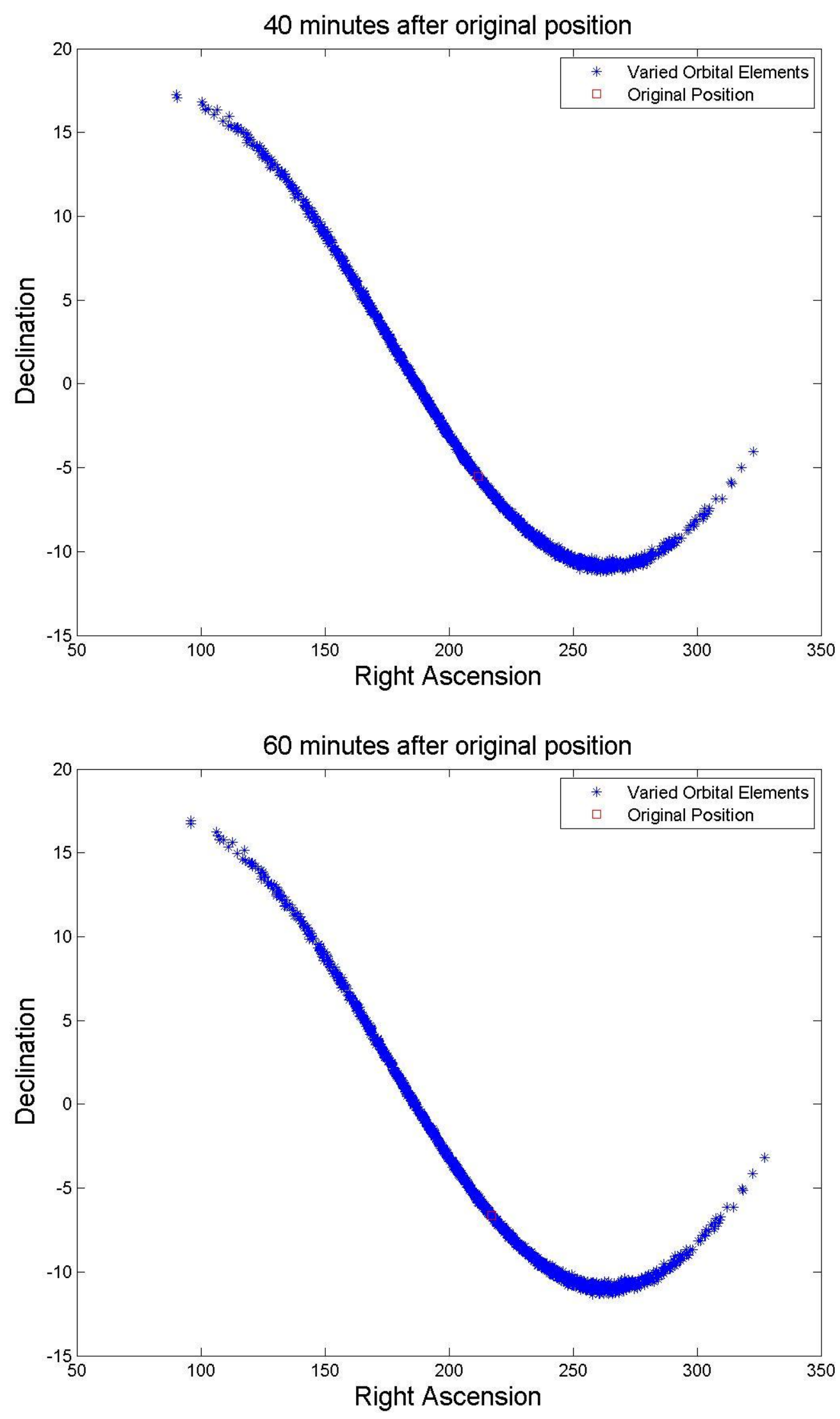


\section{Object 70318}

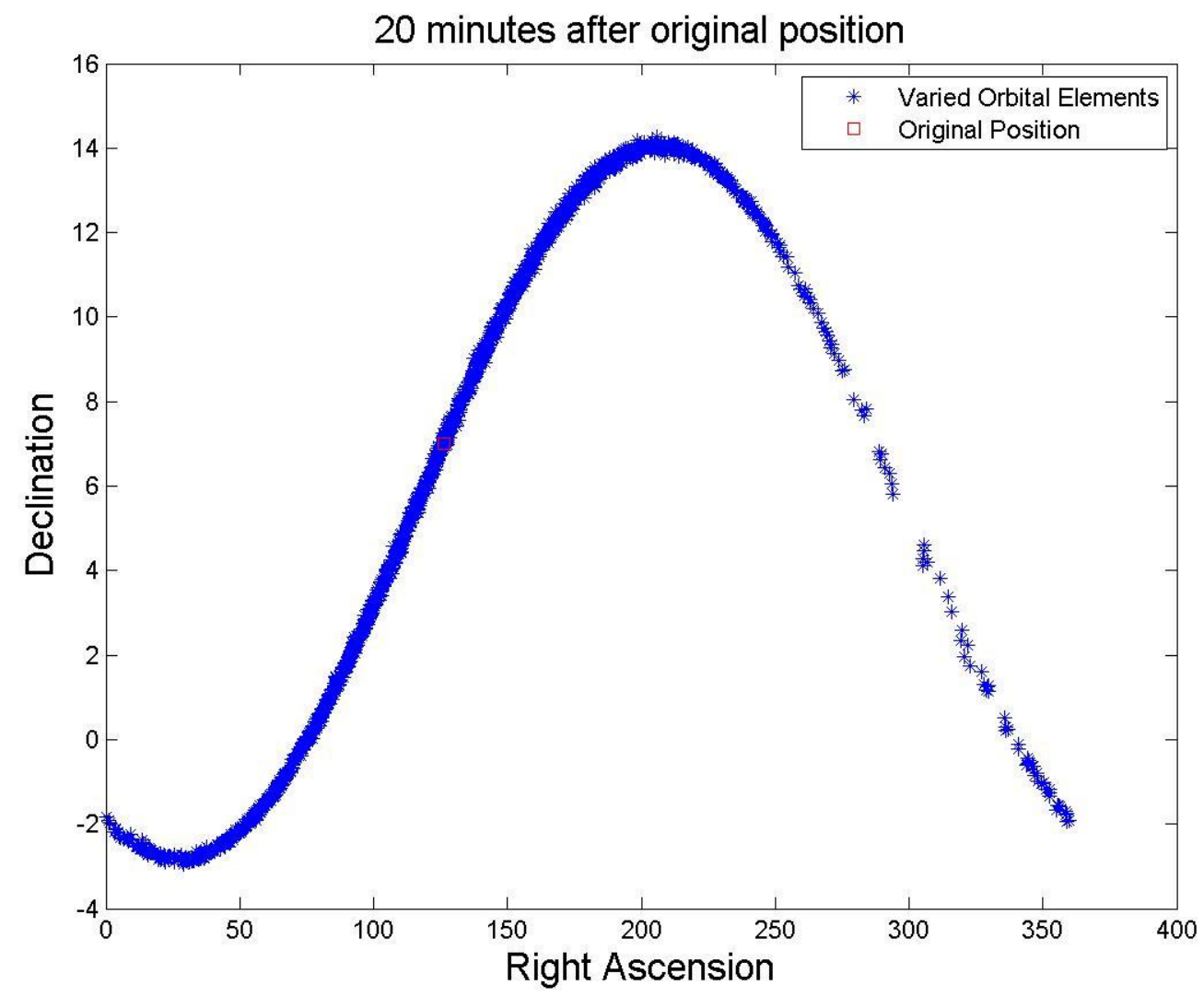



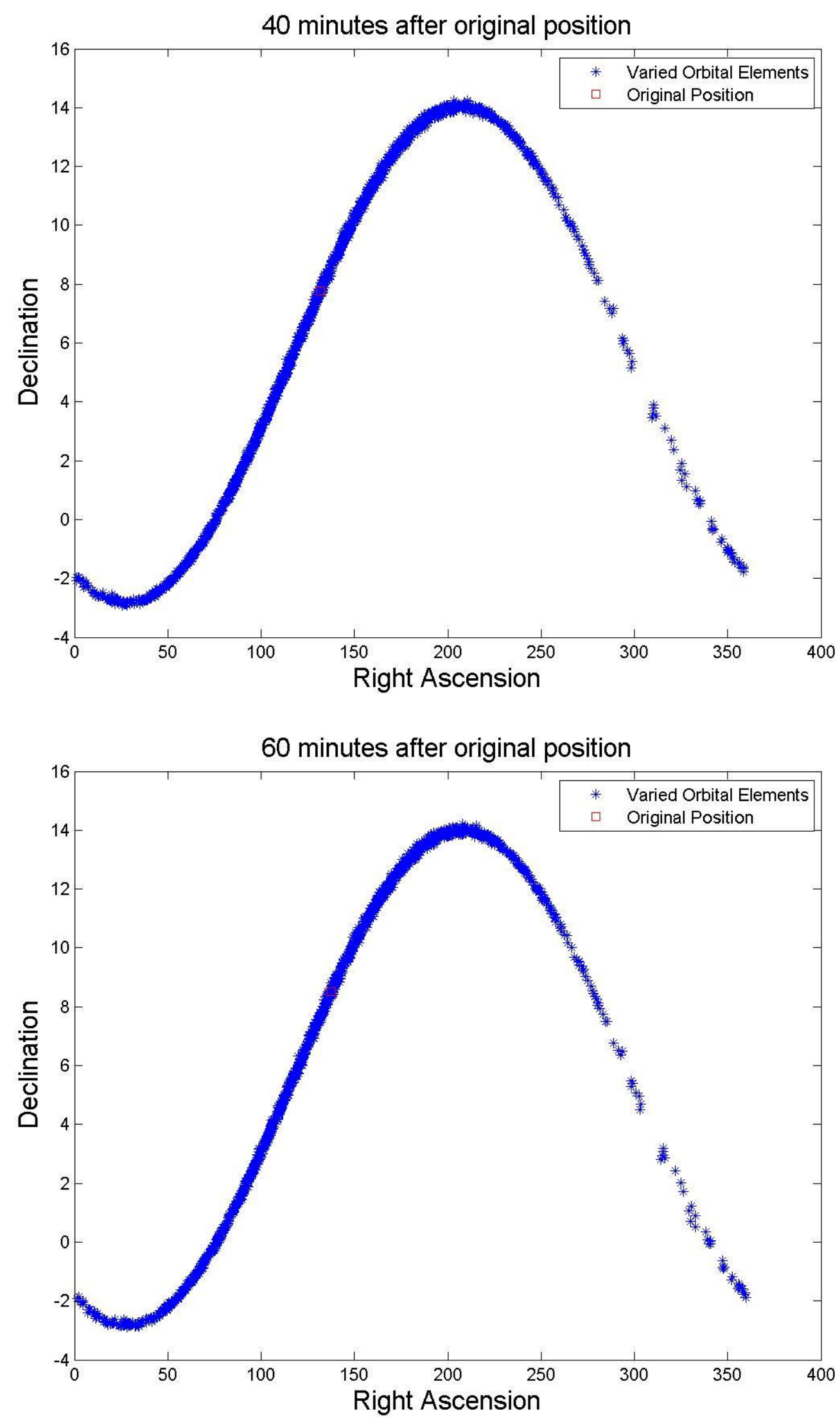


\section{Object 70319}

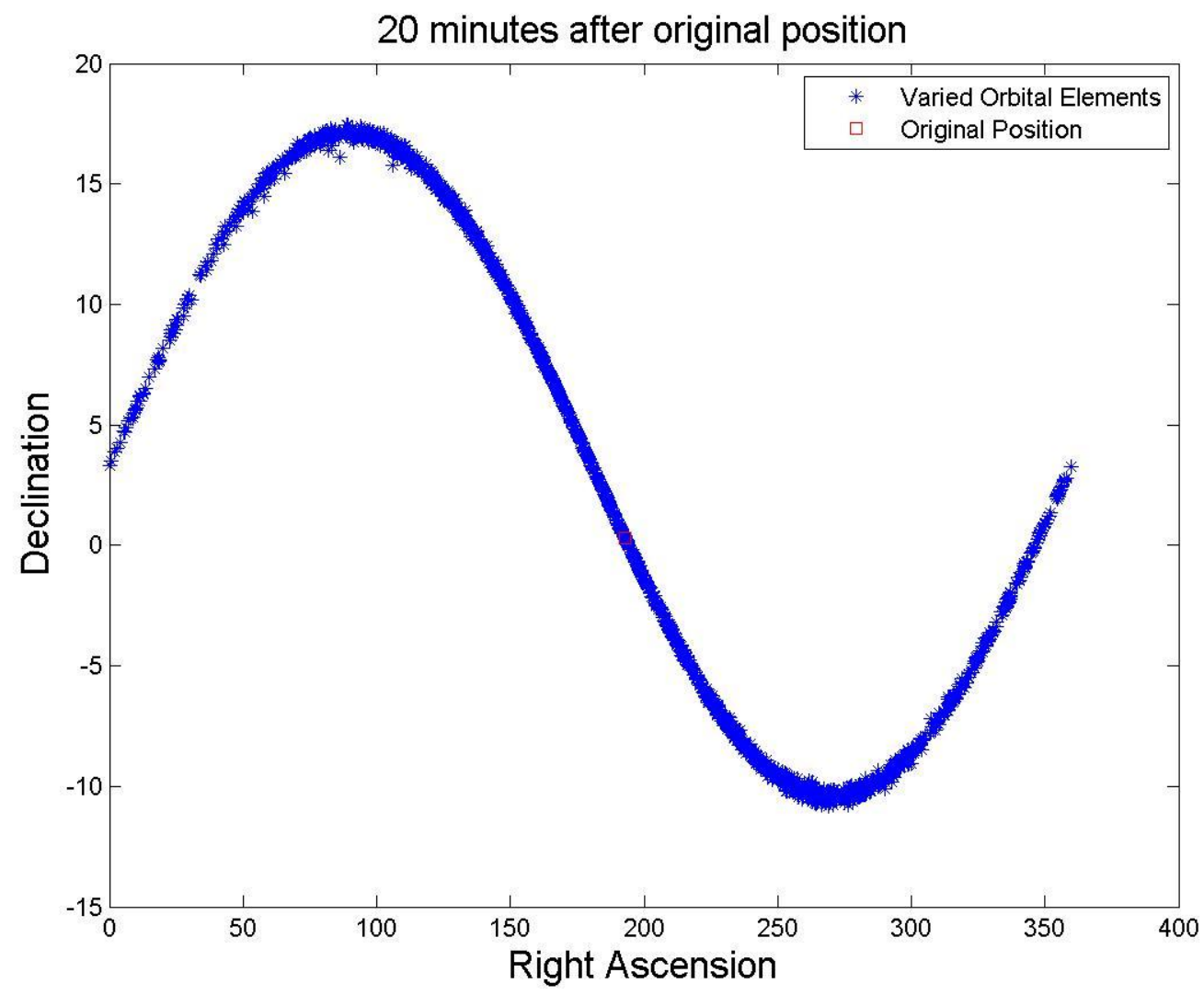



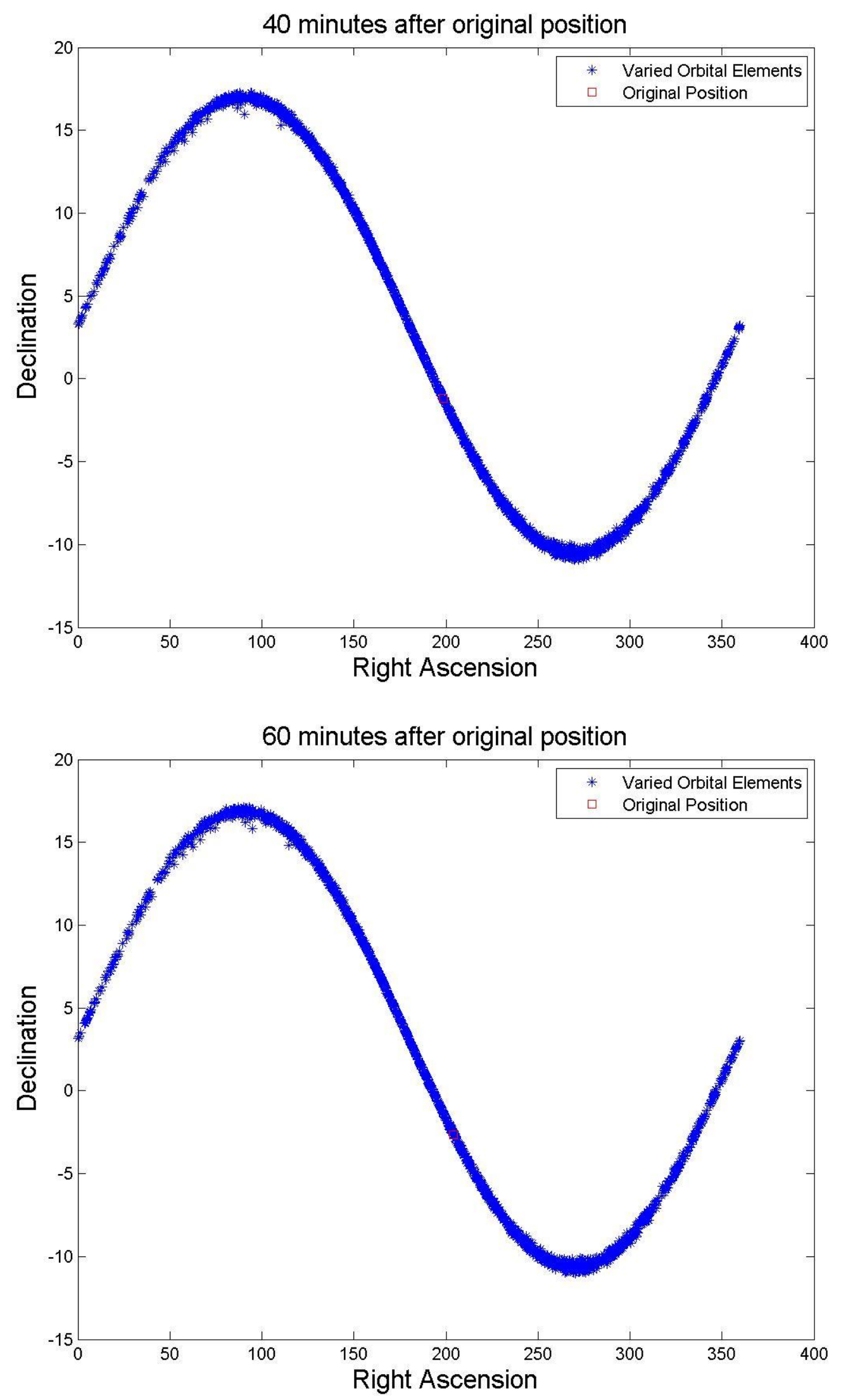


\section{Object 70320}

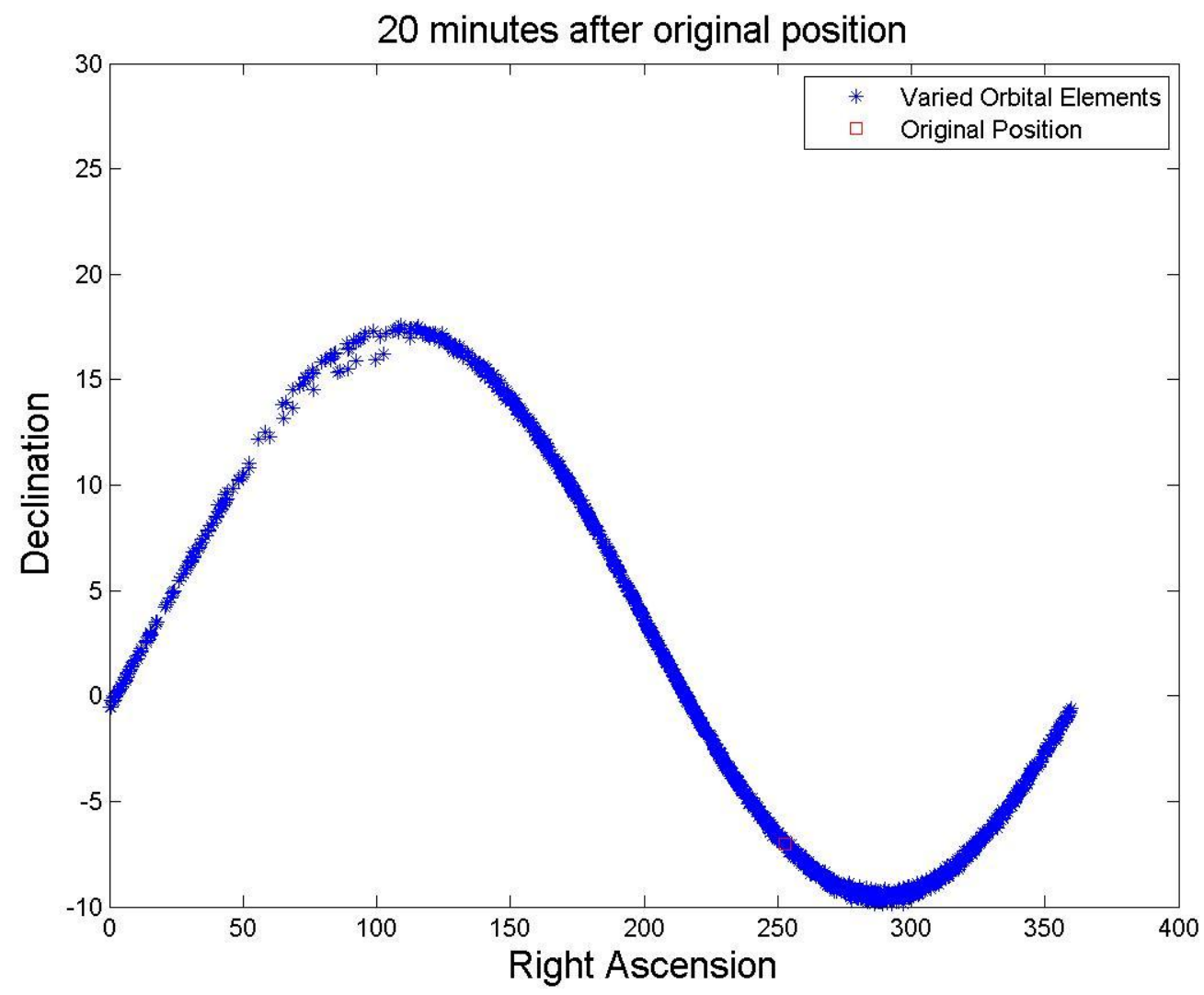



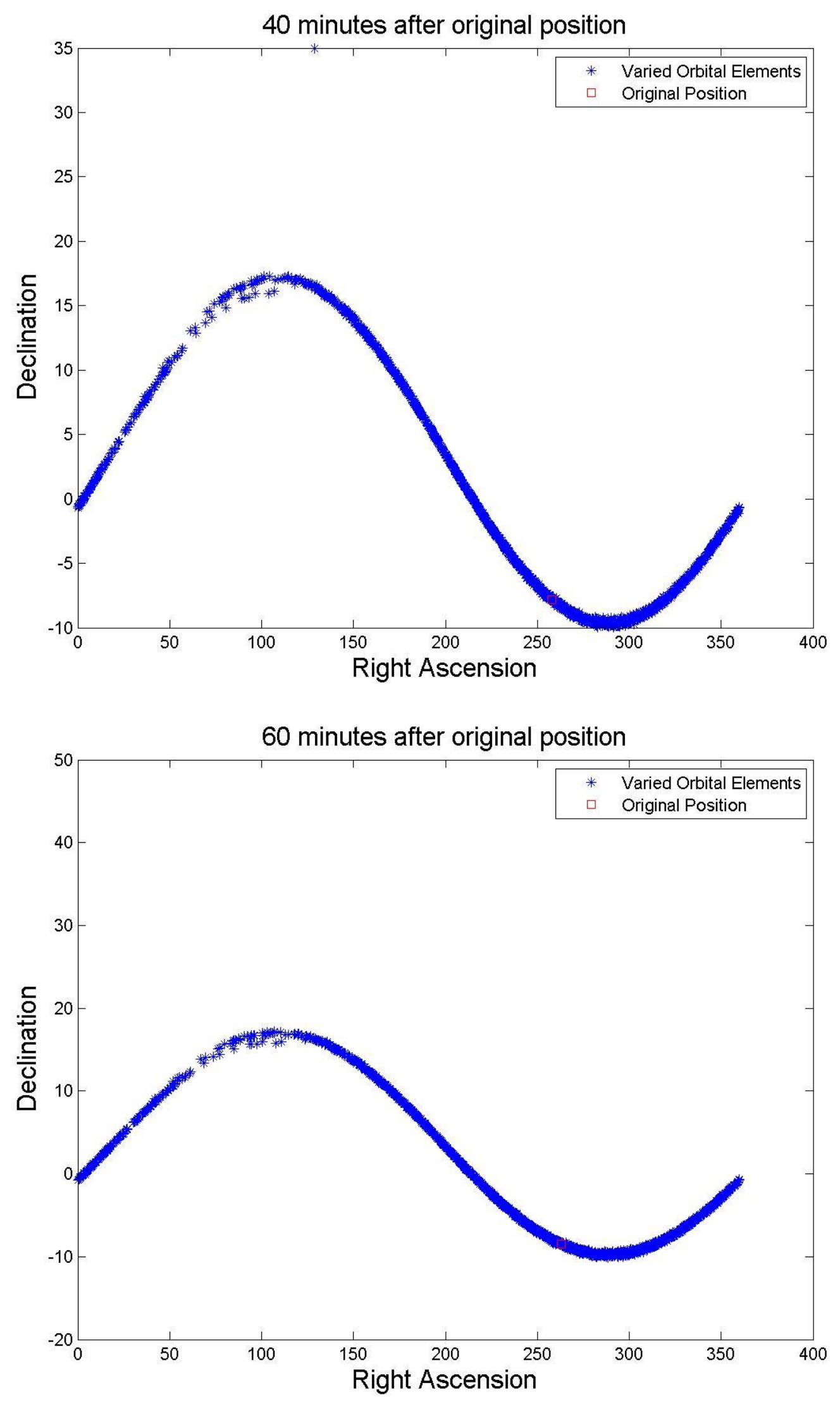


\section{Object 70321}

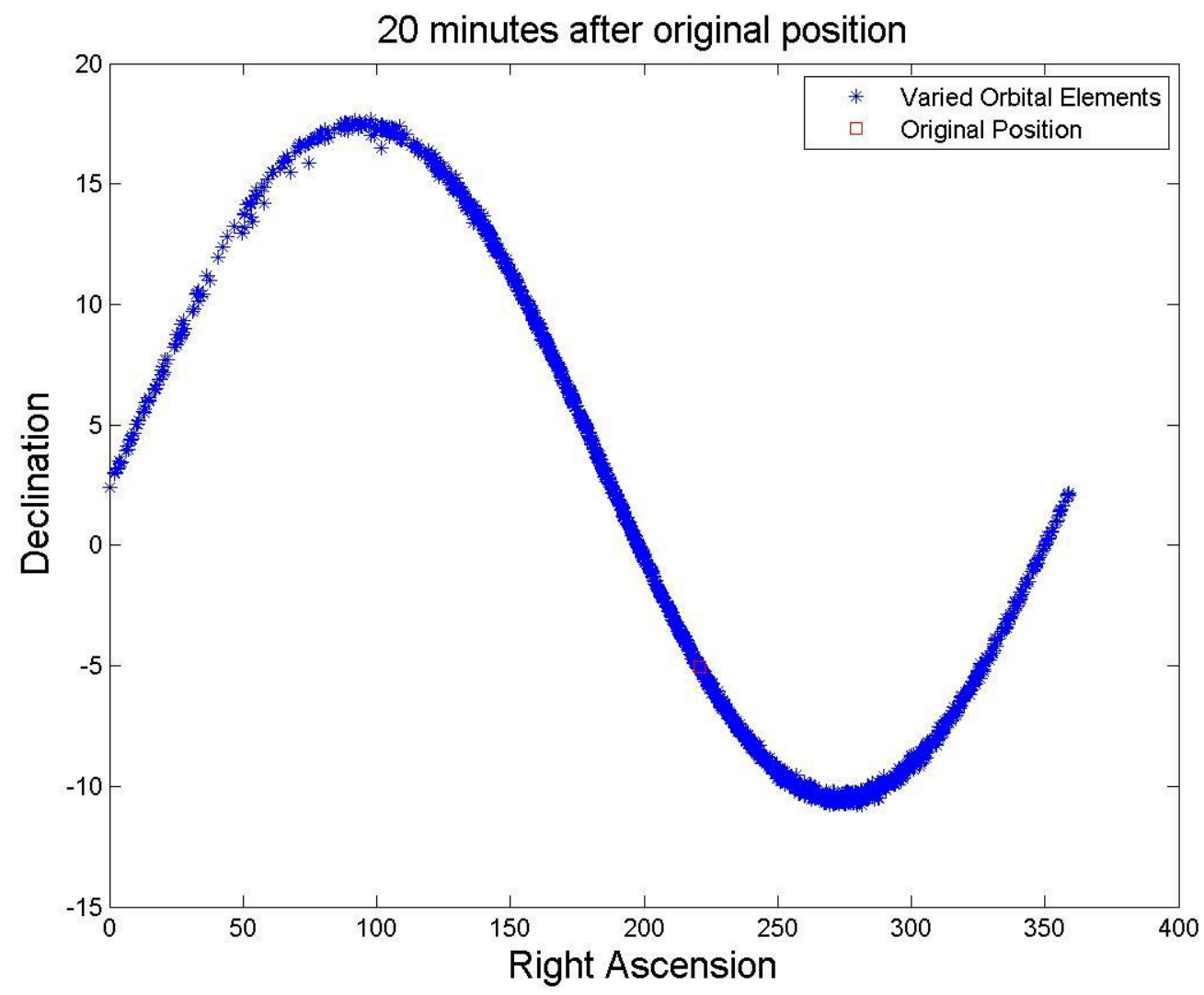



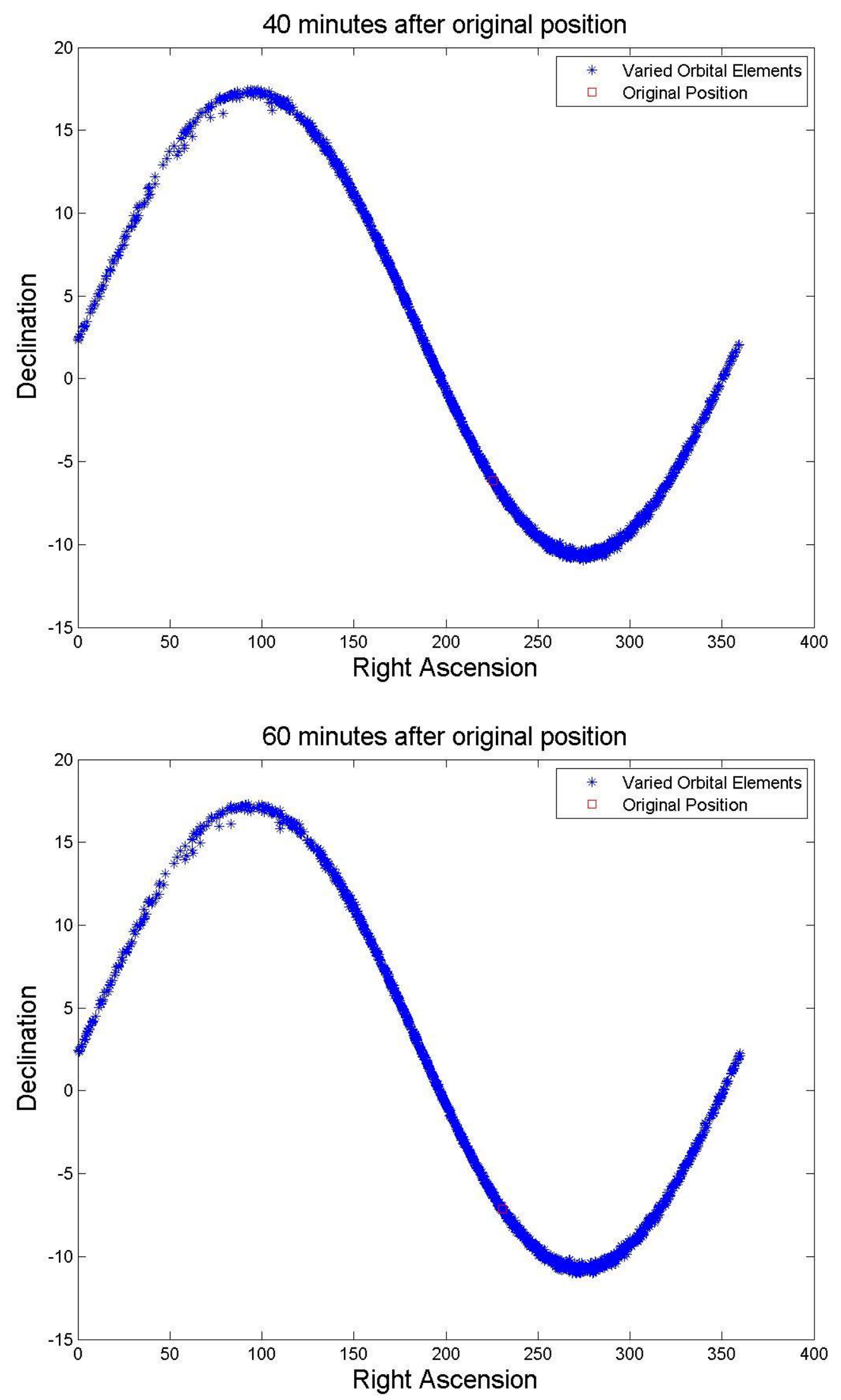


\section{Object 70322}

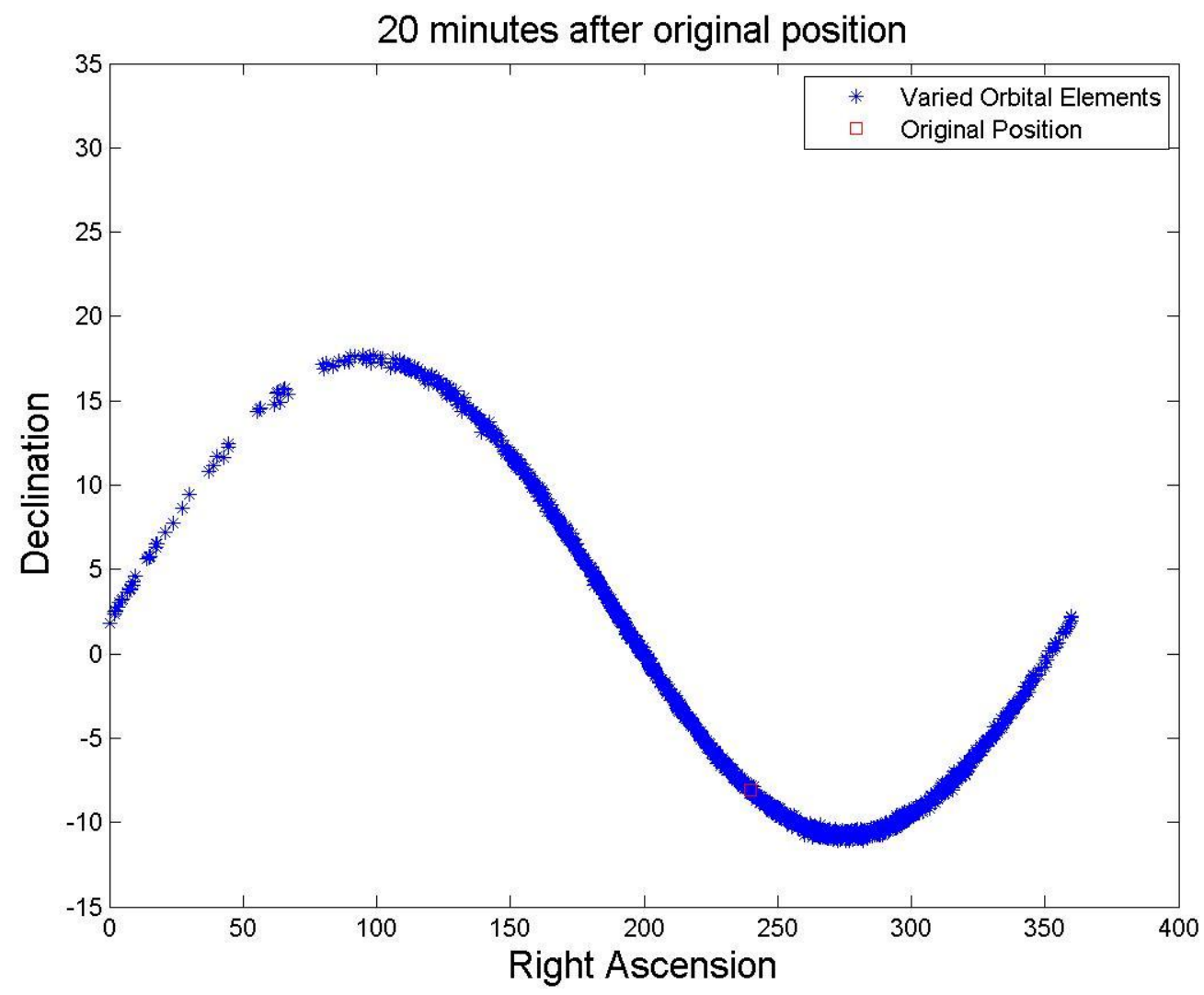



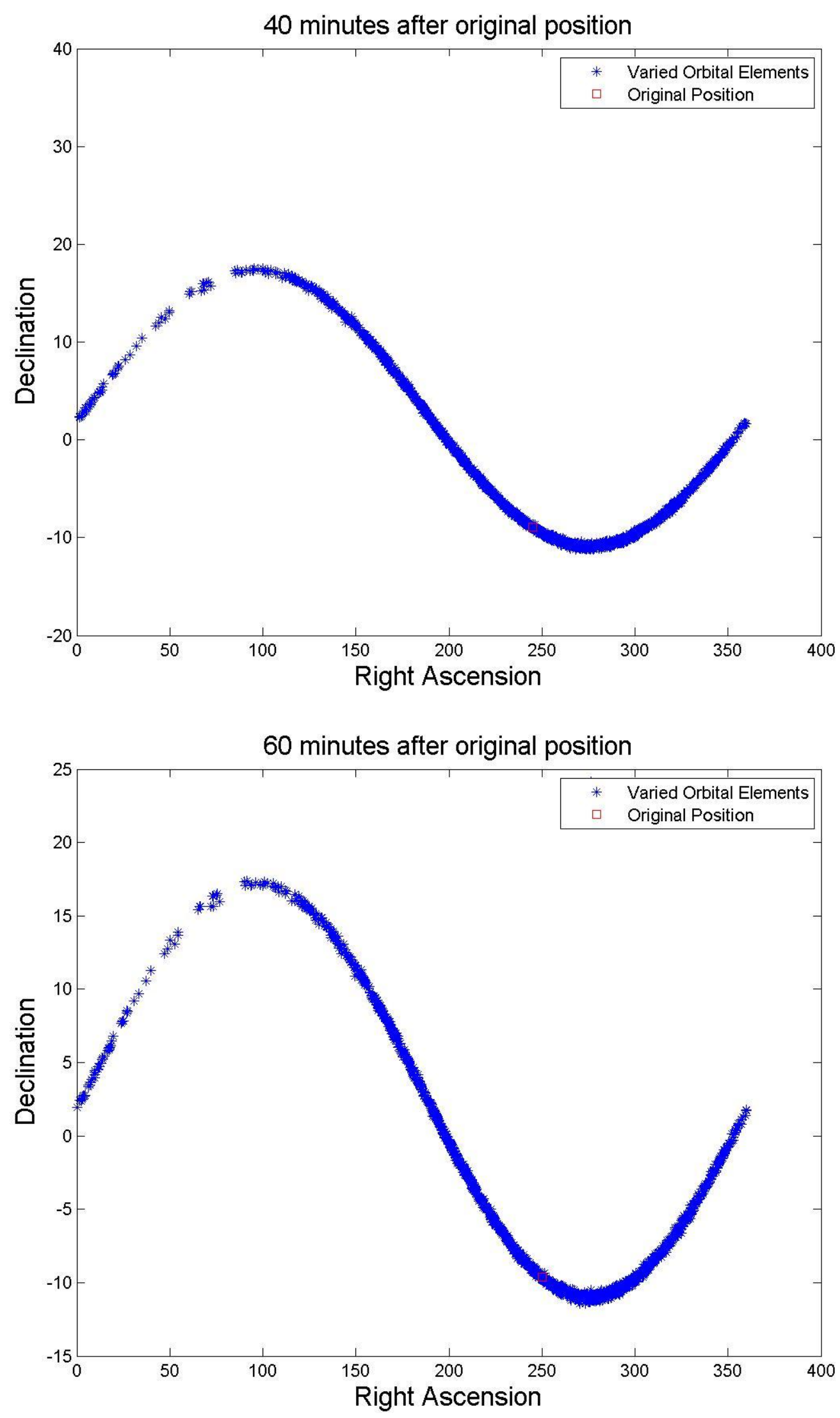


\section{Object 70323}

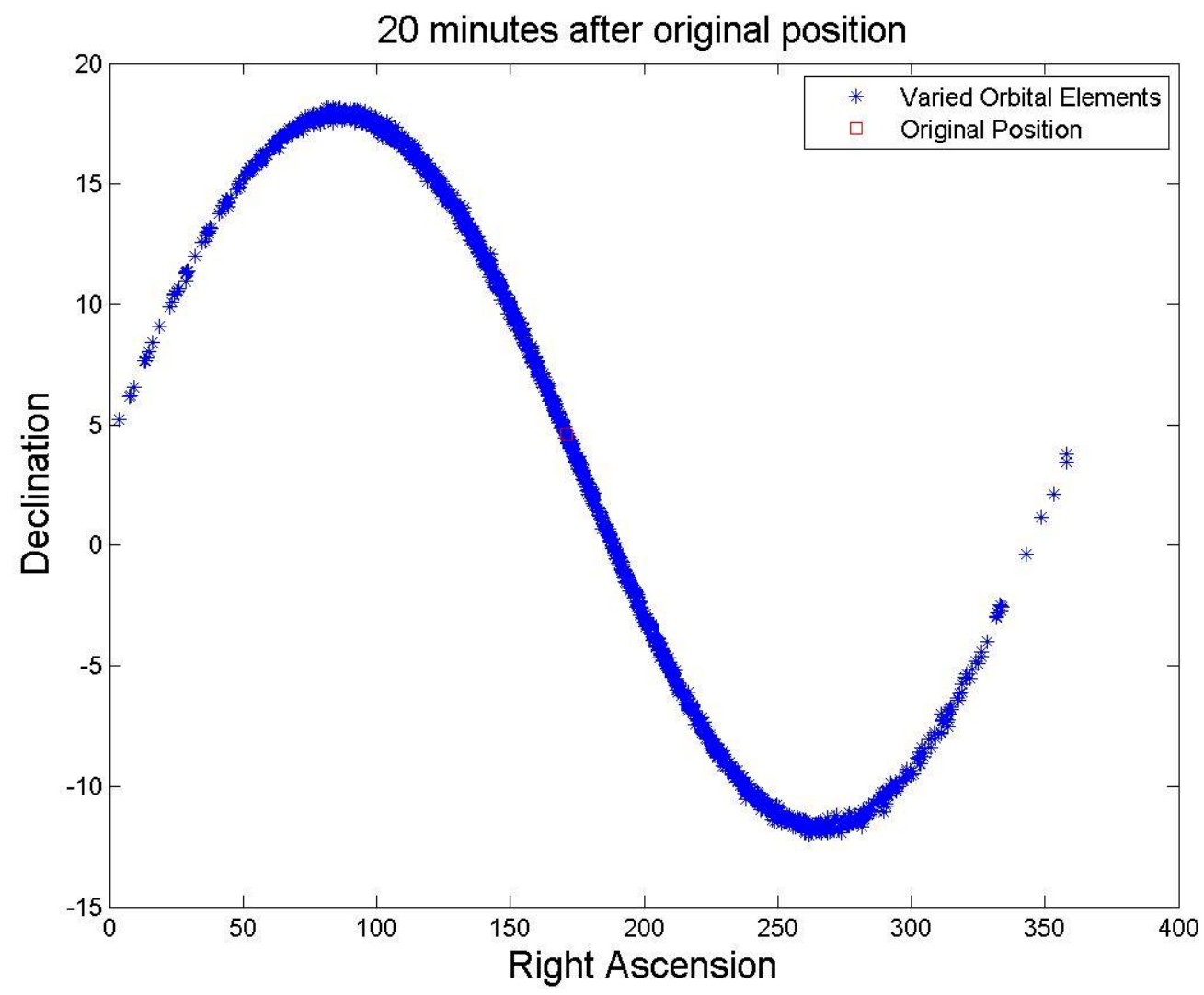



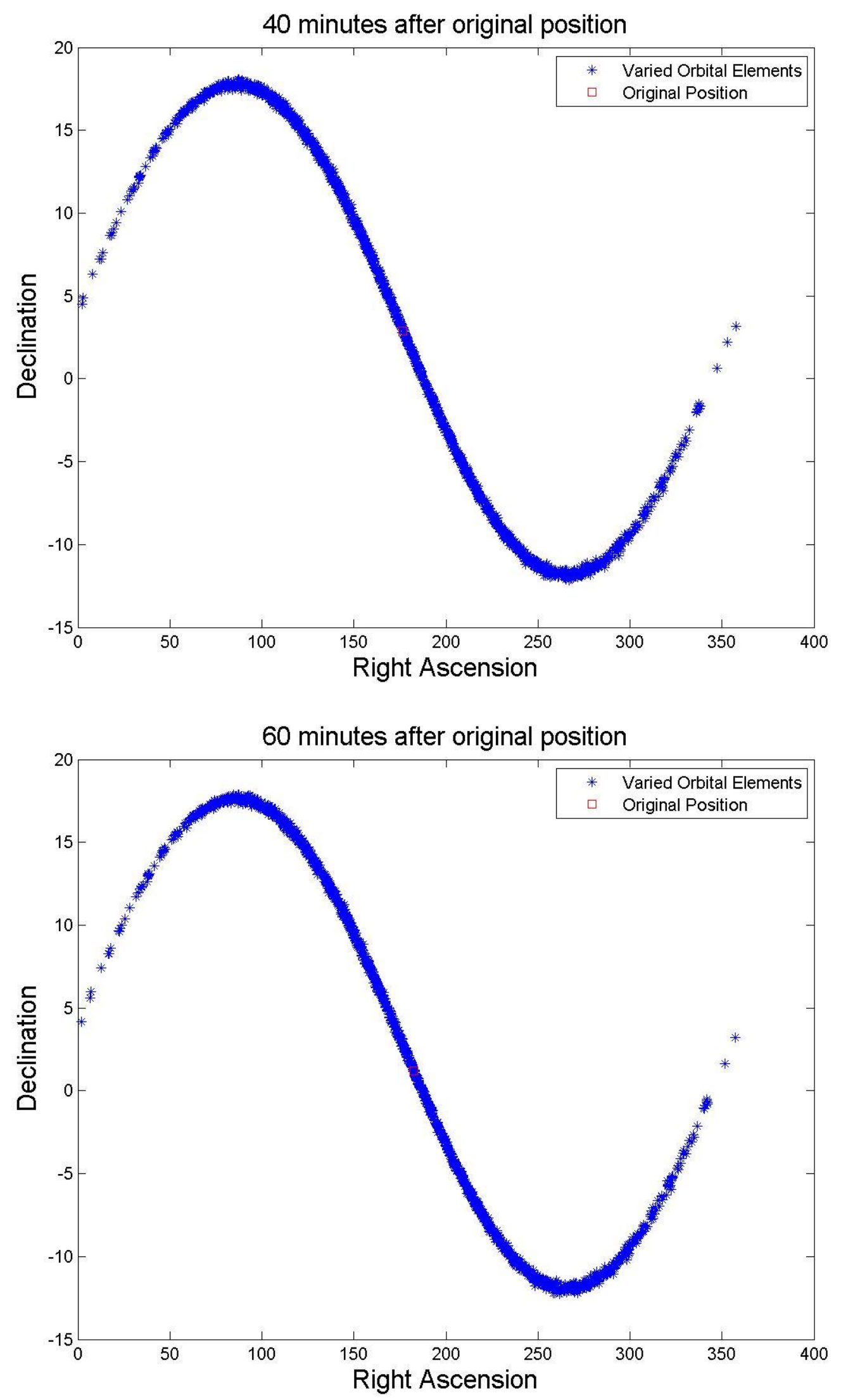


\section{Object 70324}

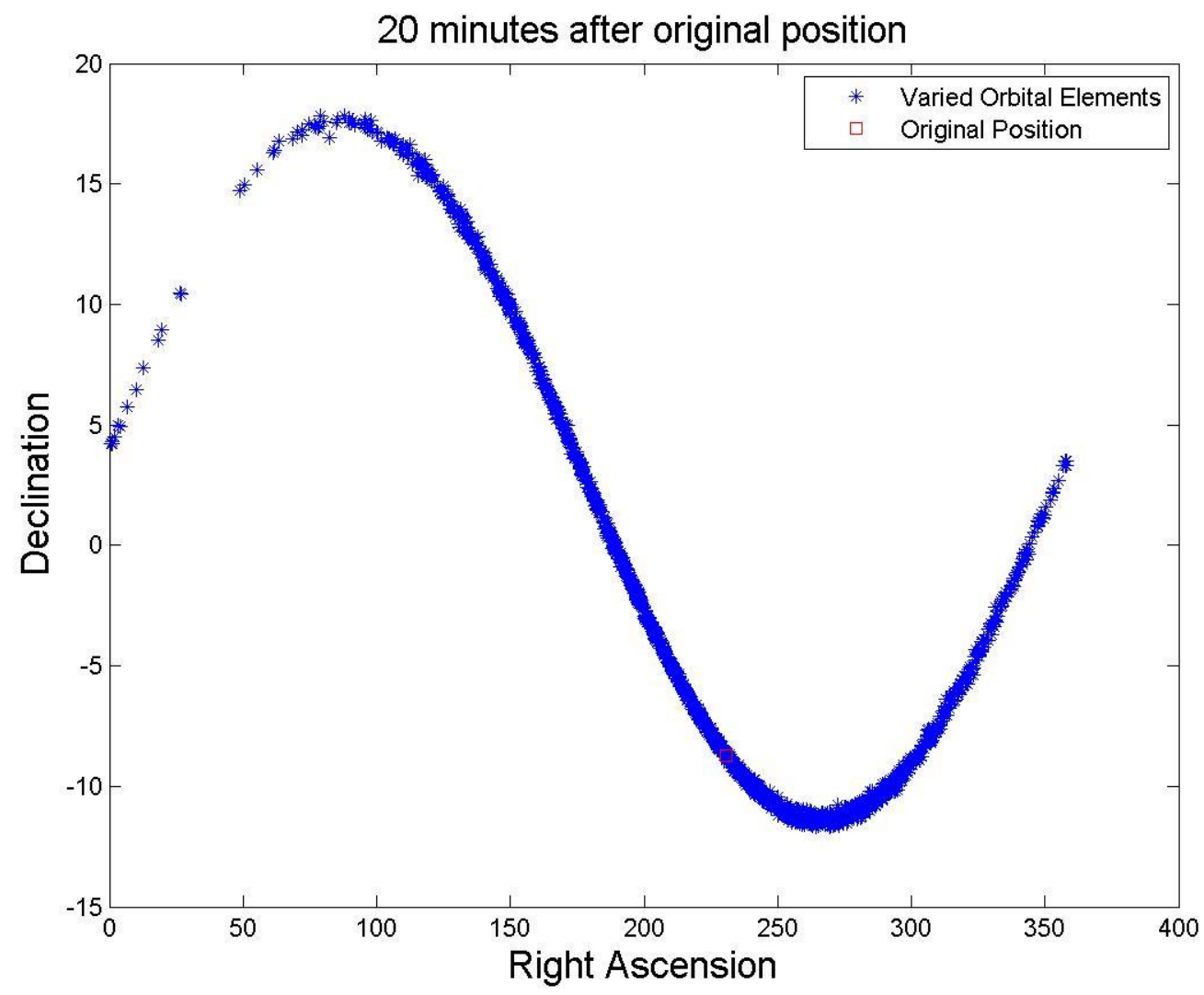



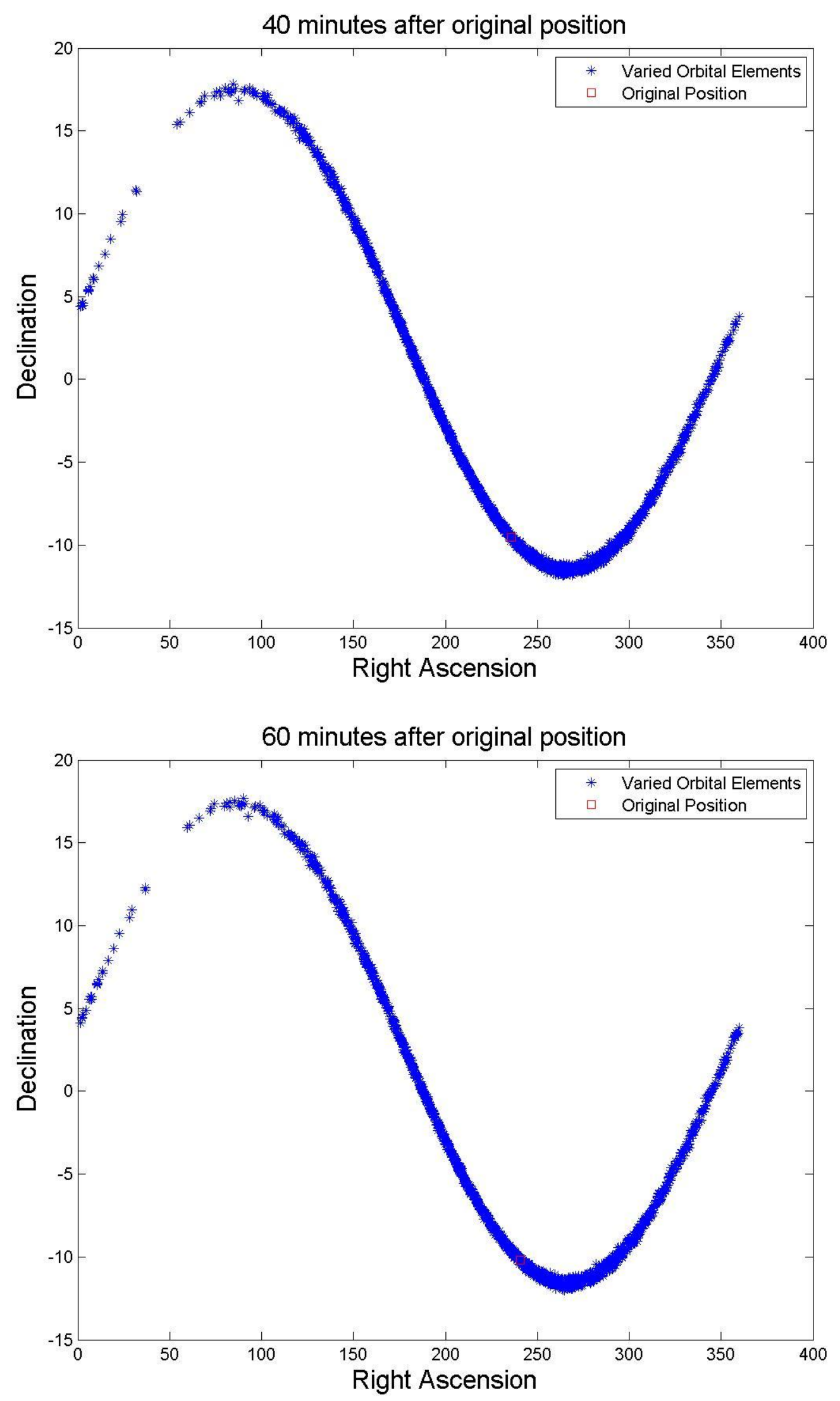


\section{Object 70325}

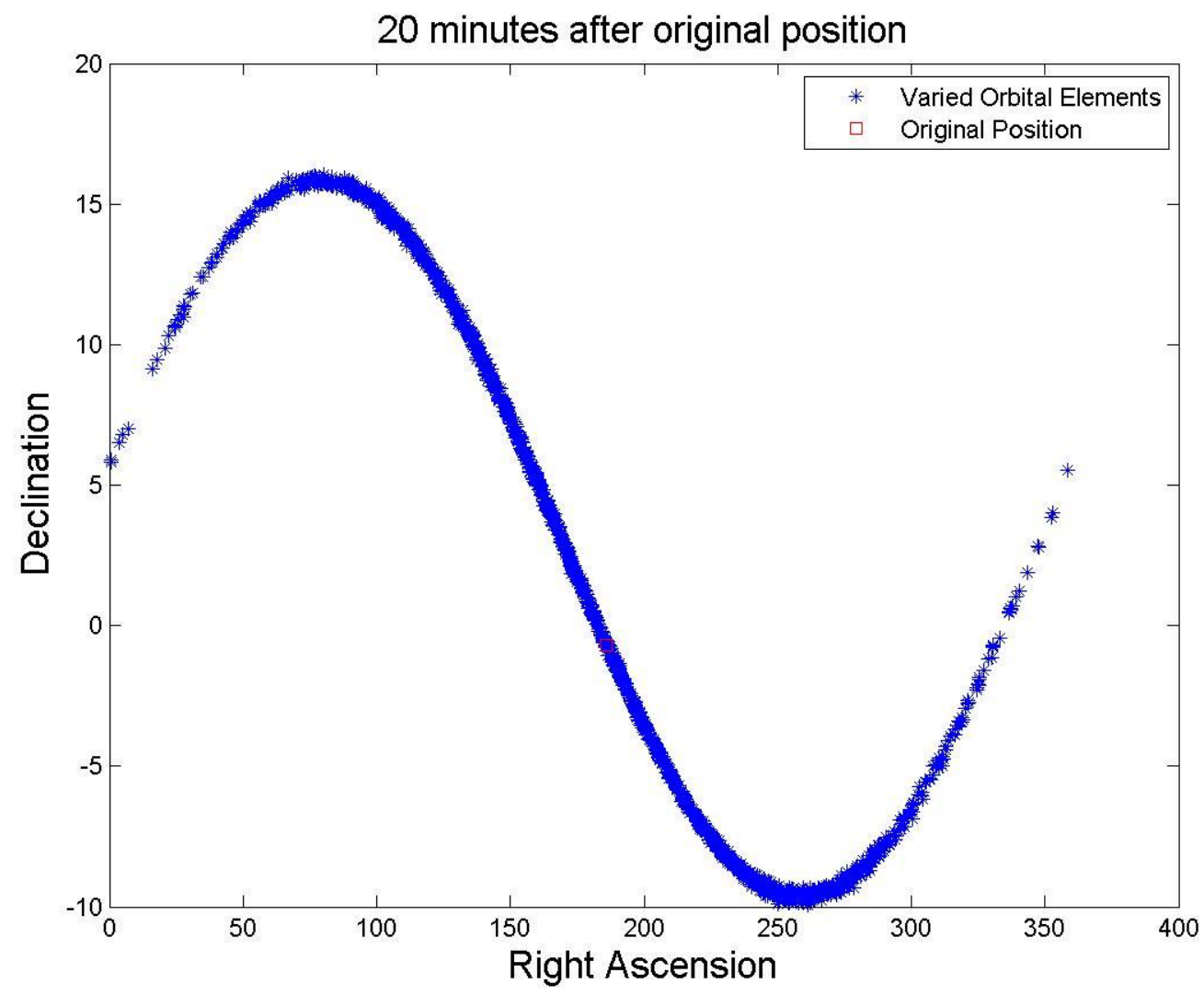



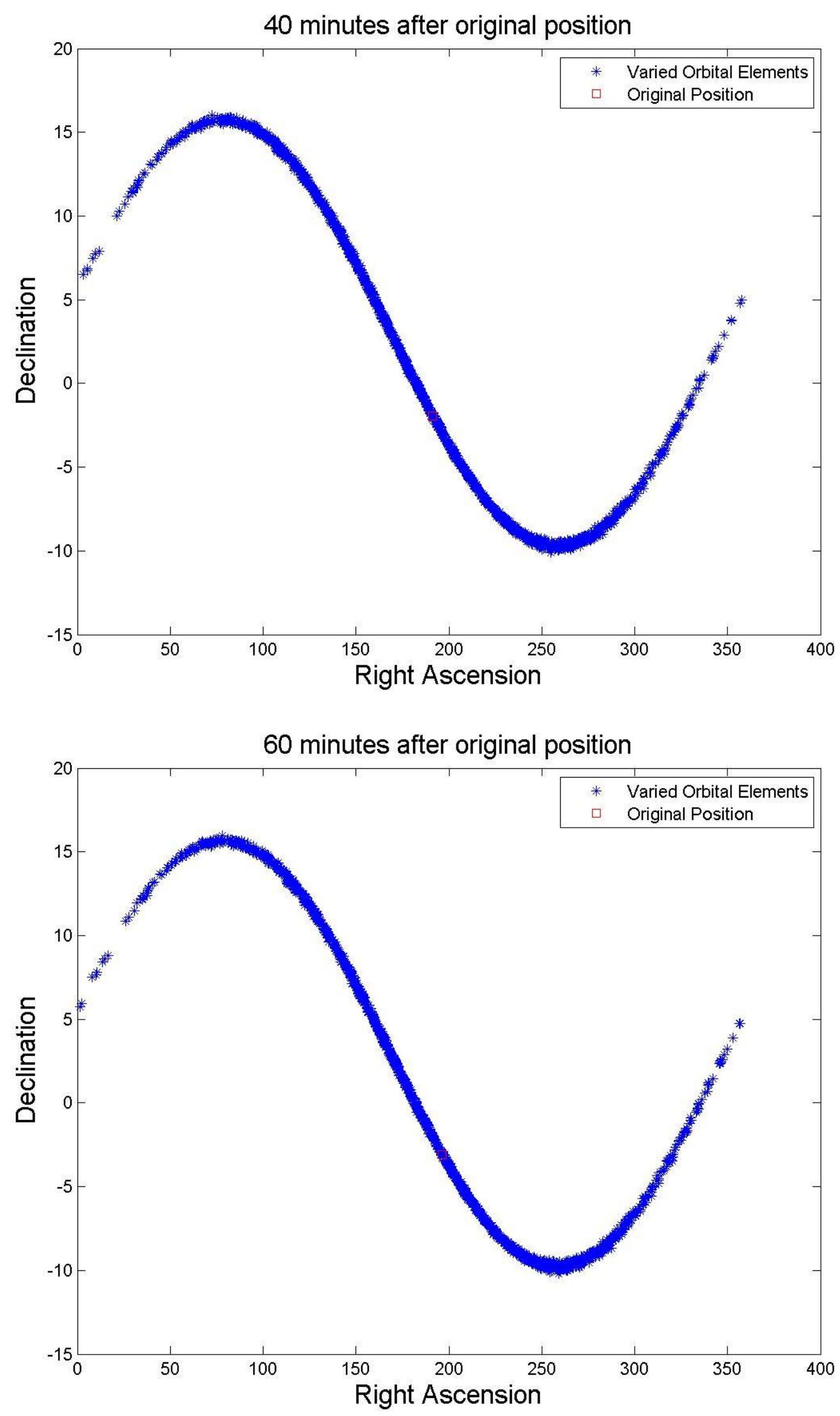


\section{Object 70326}

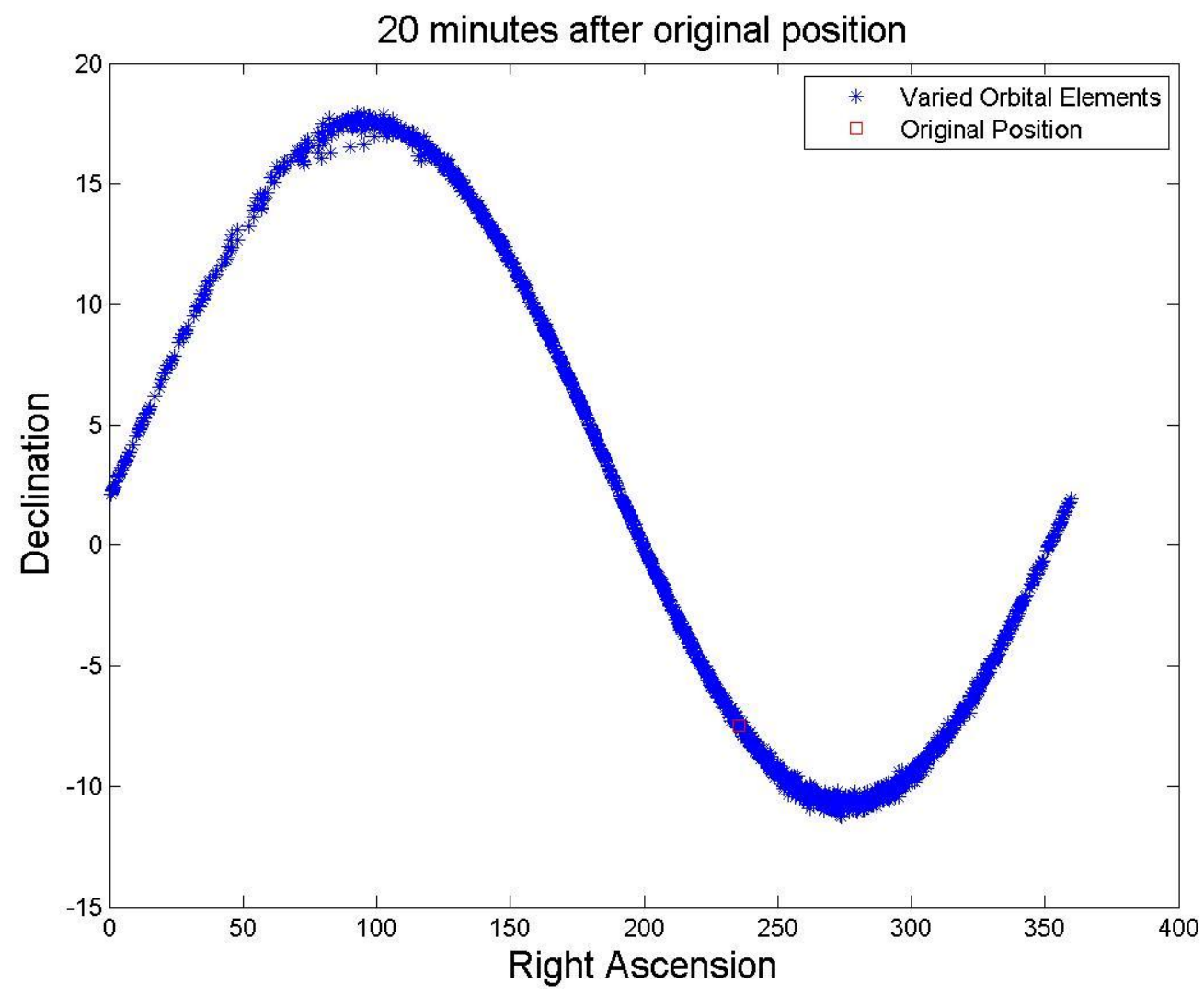



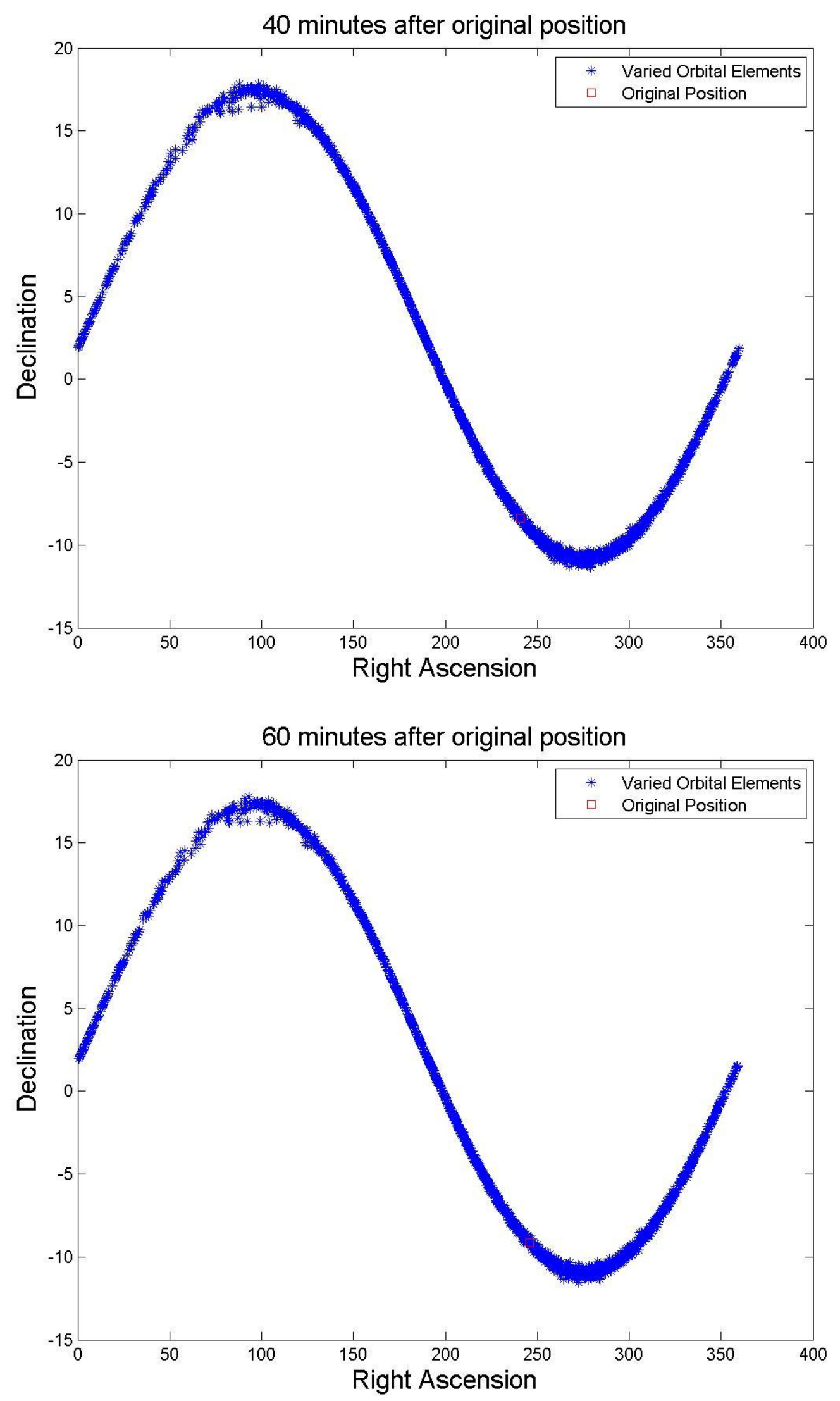


\section{Object 70327}

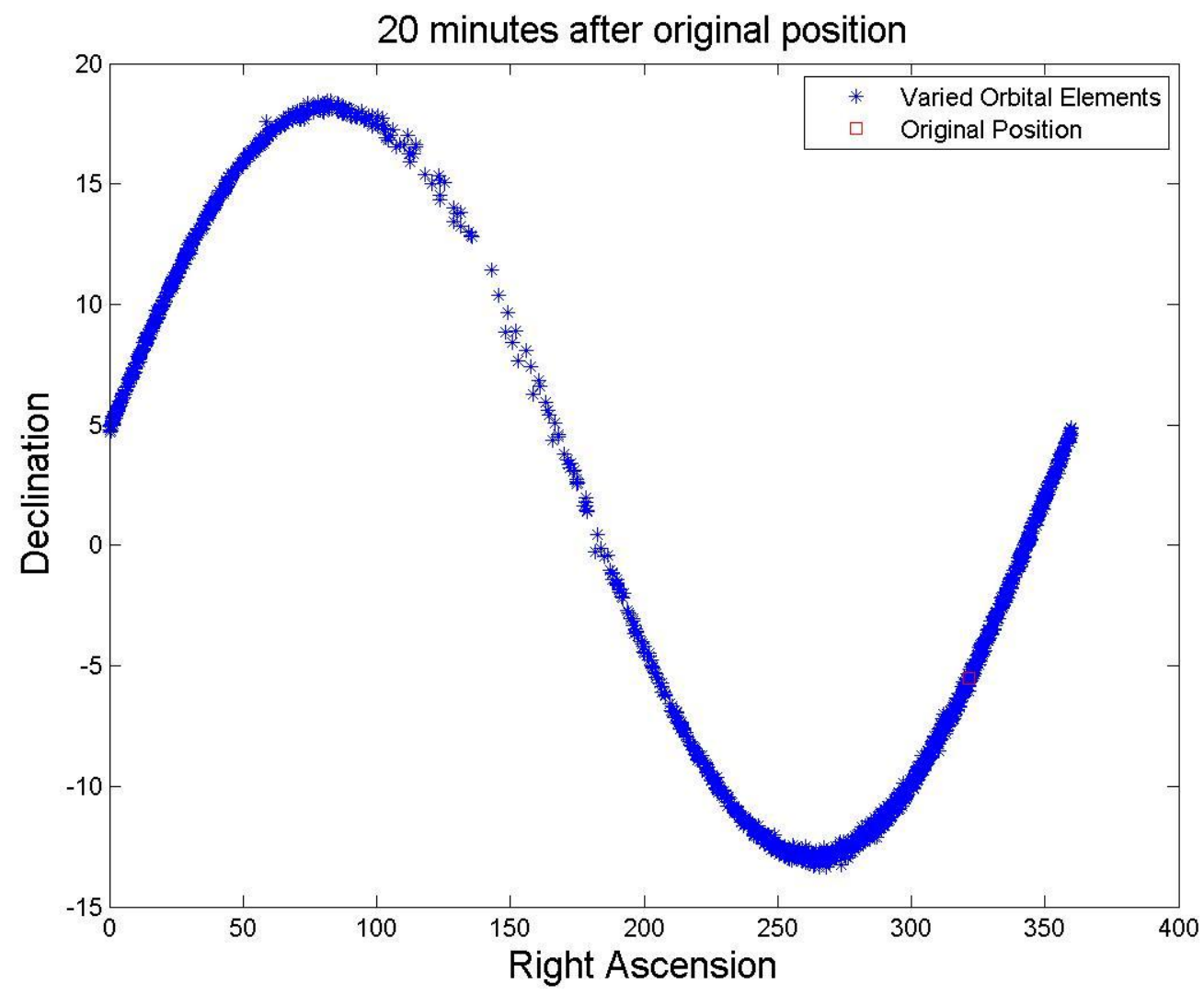



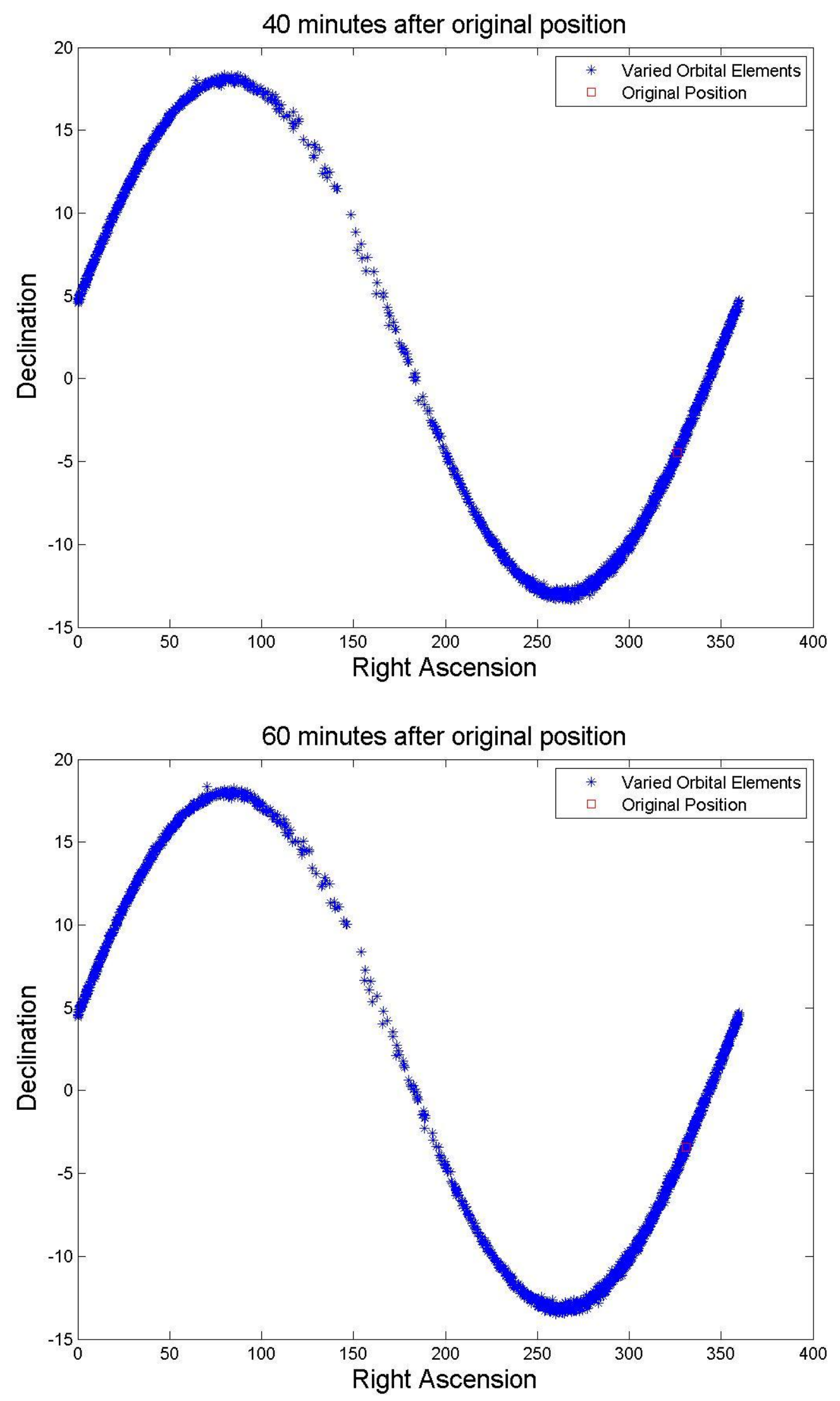


\section{Object 70328}

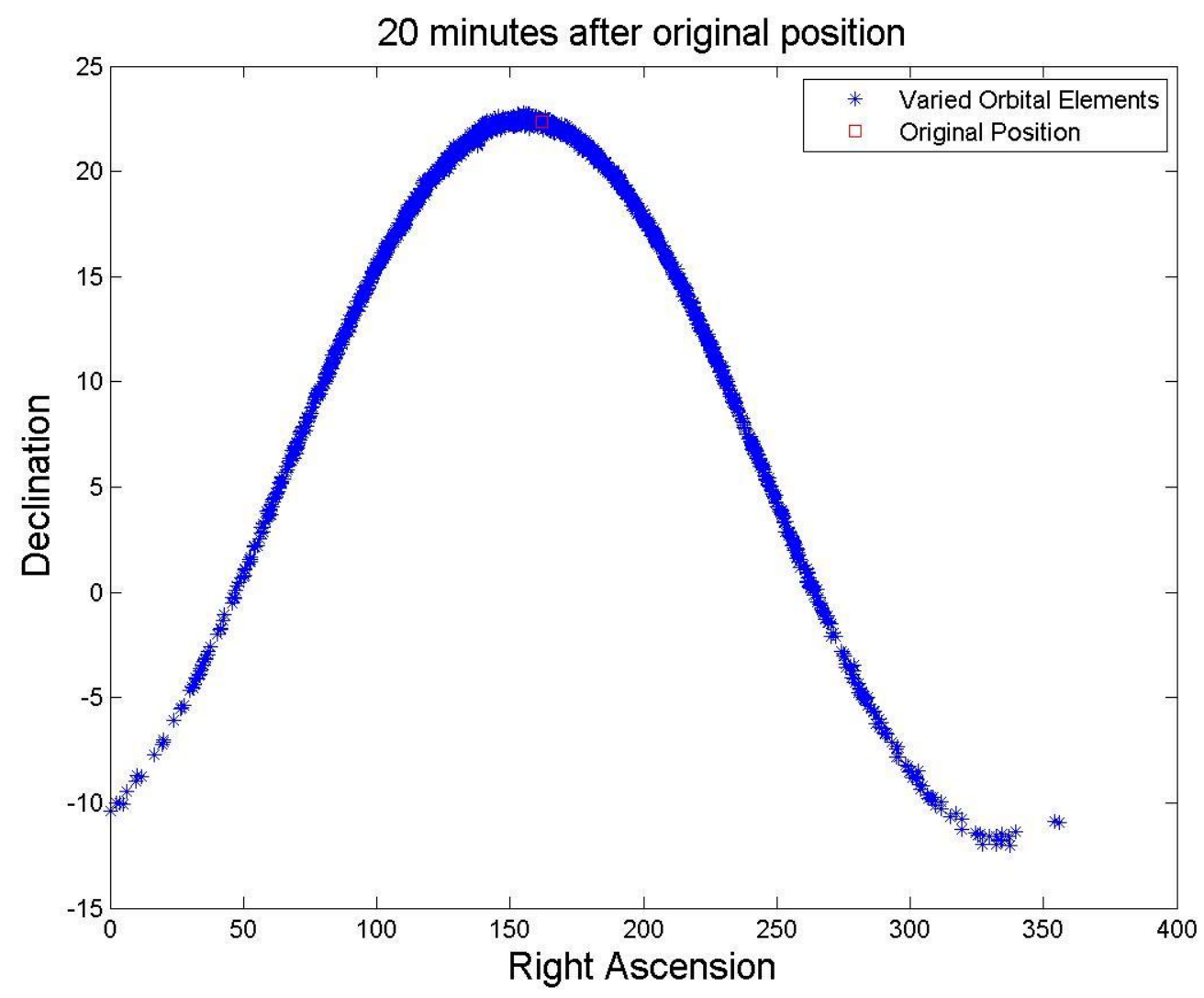



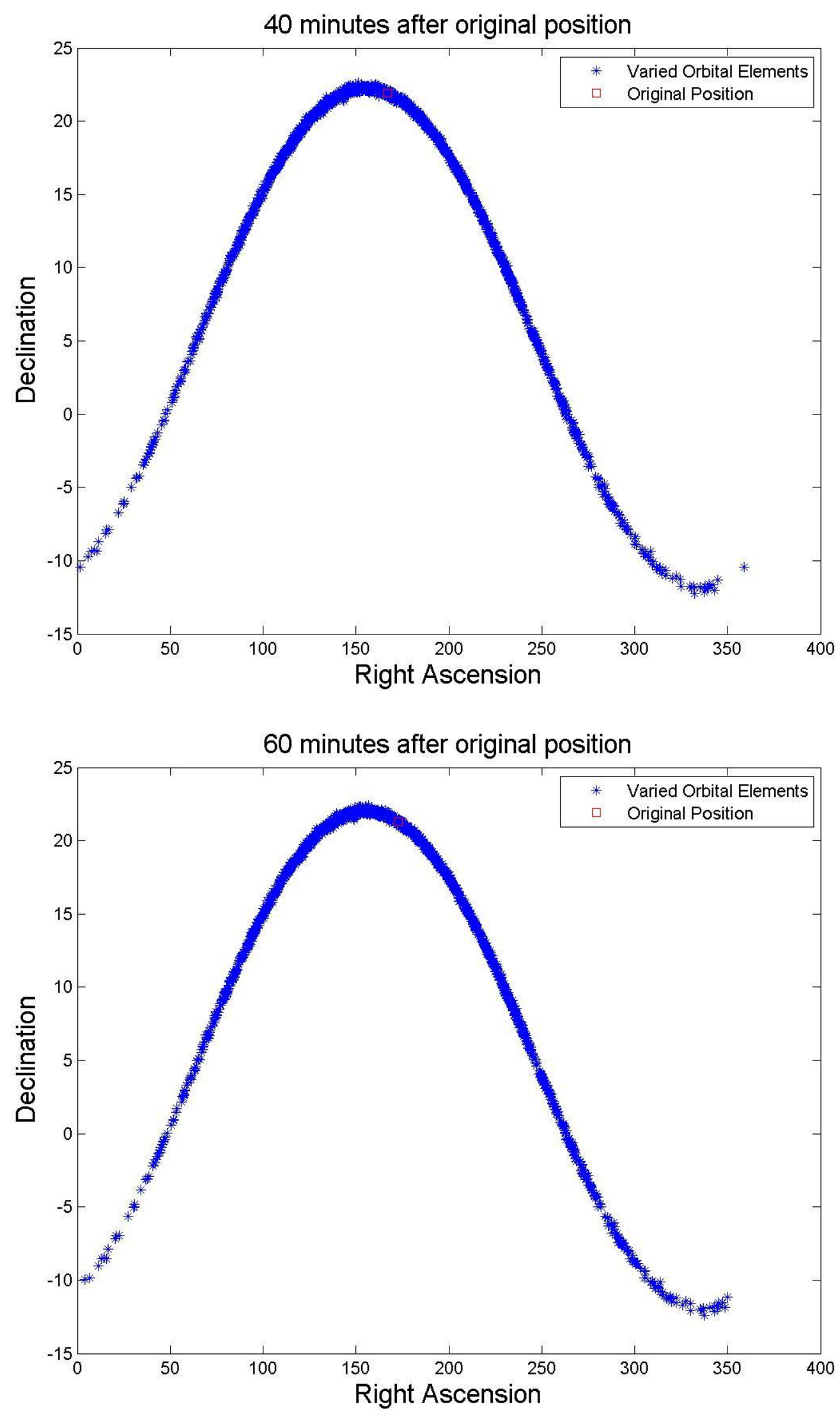


\section{Object 70329}

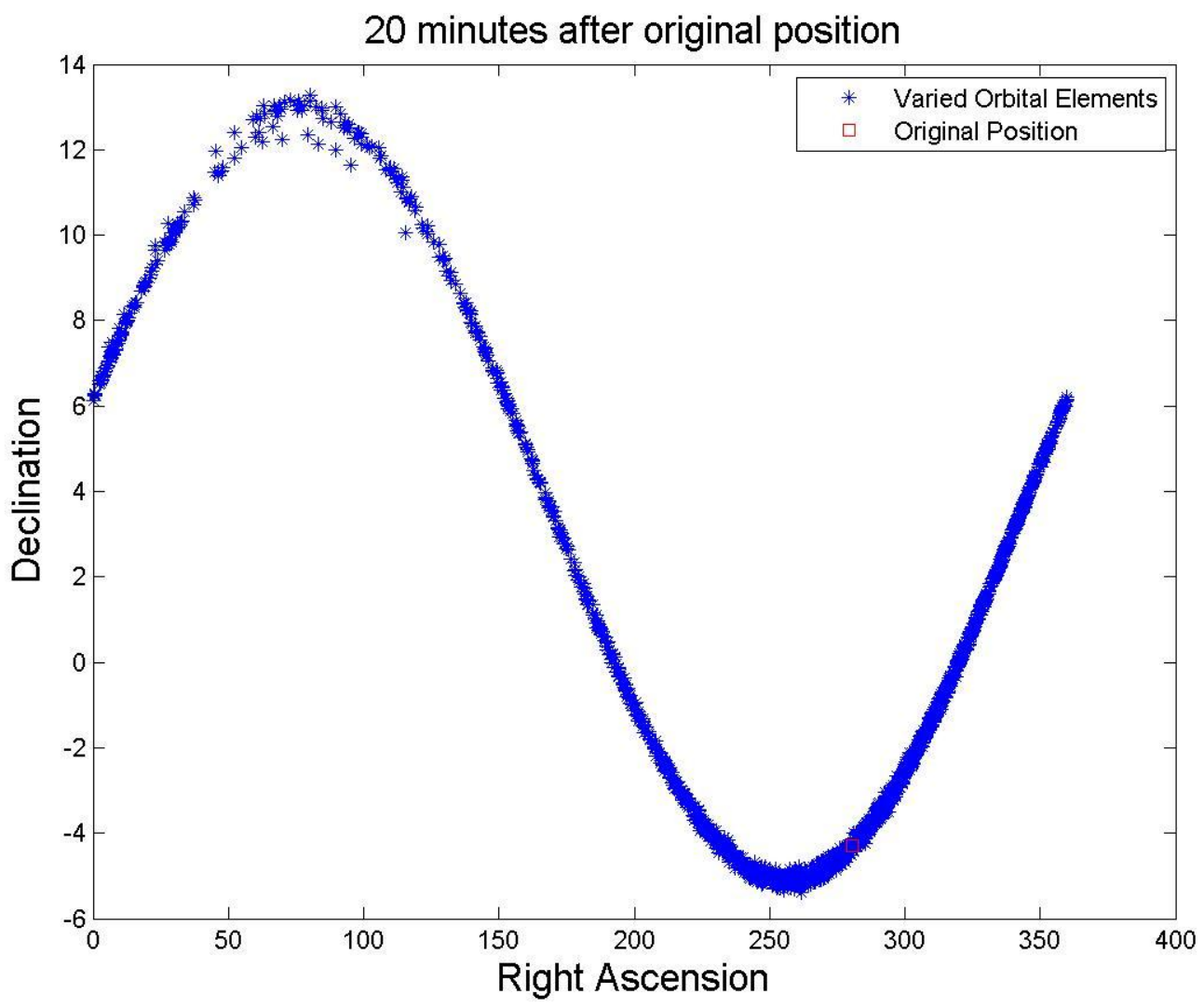



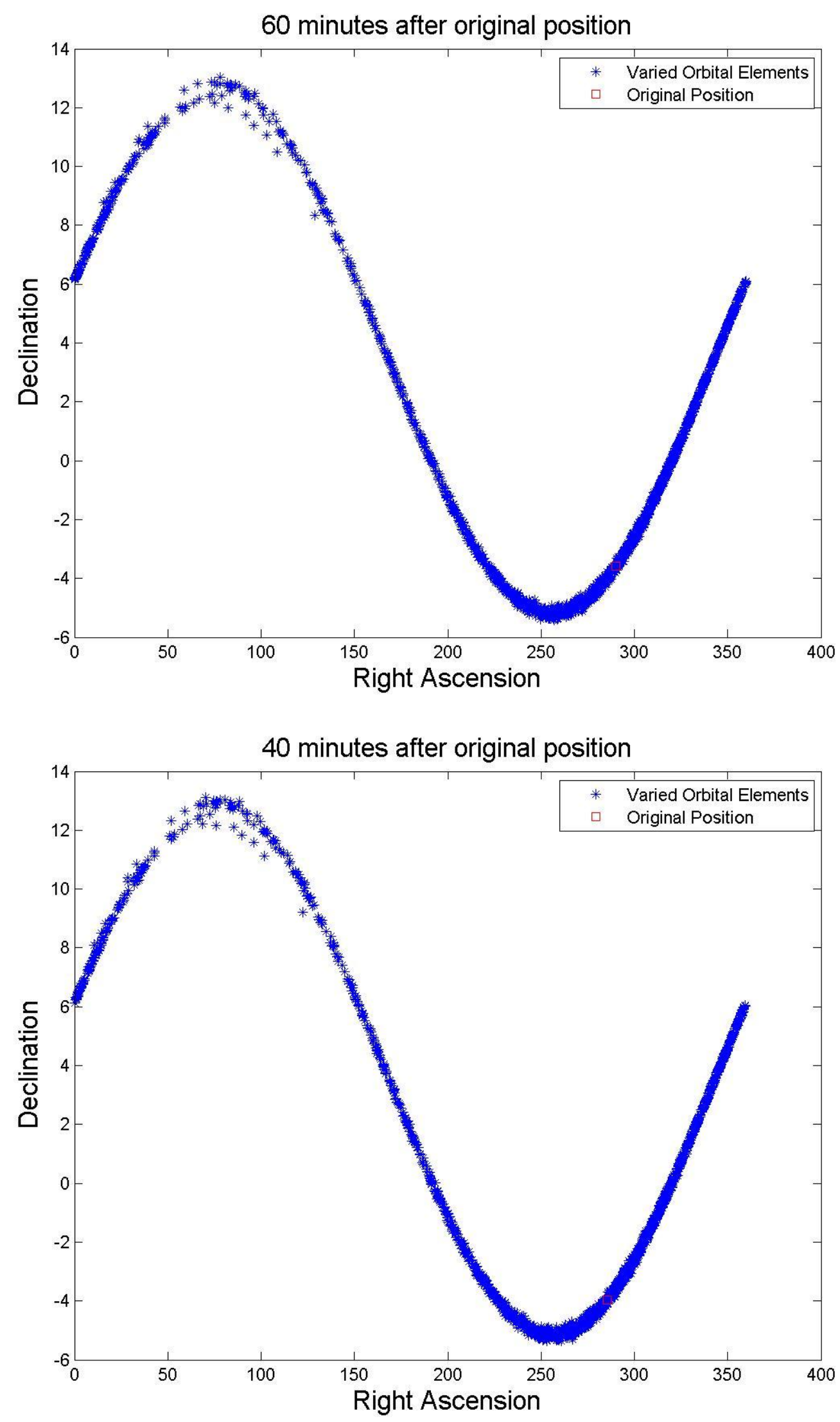


\section{Appendix D: 2009 Graphs}

Object 70701

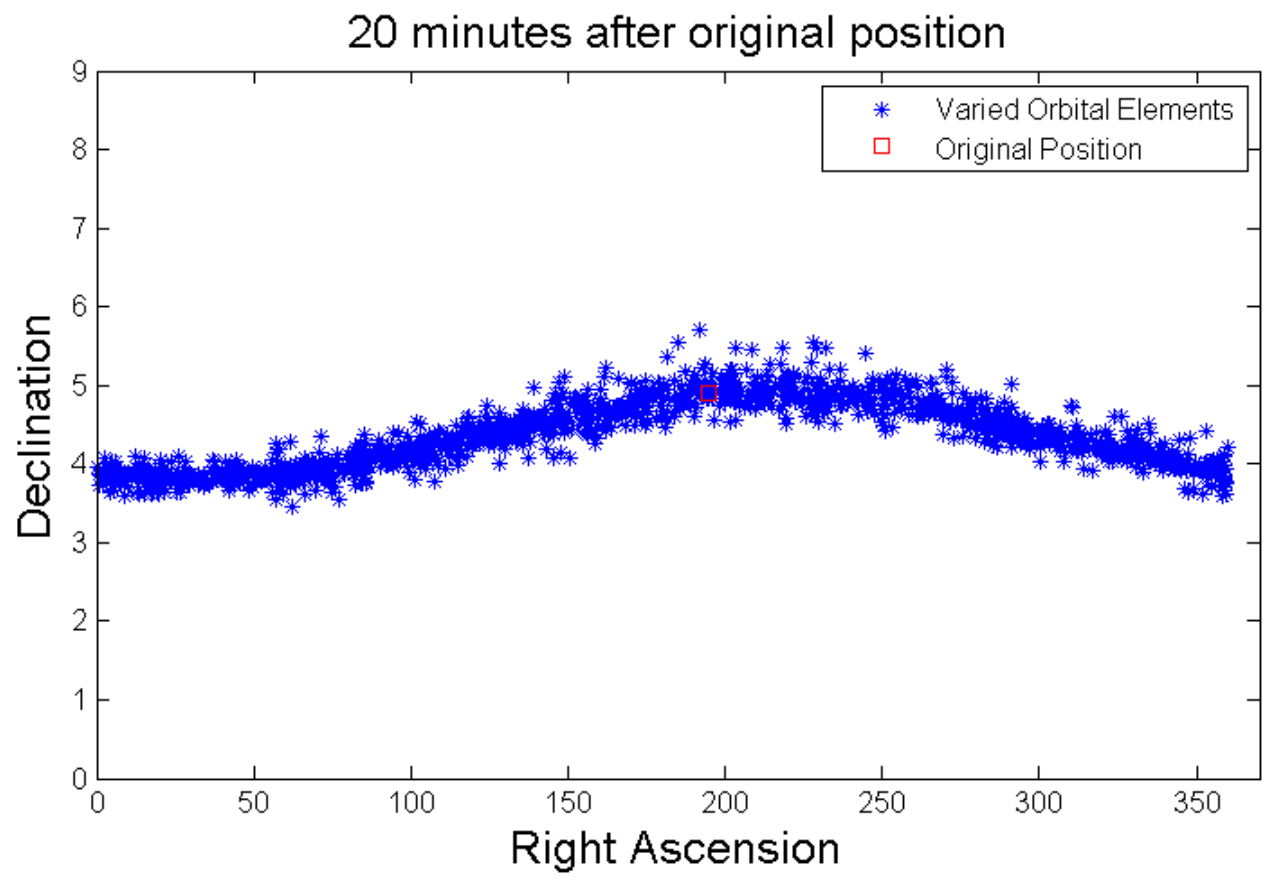



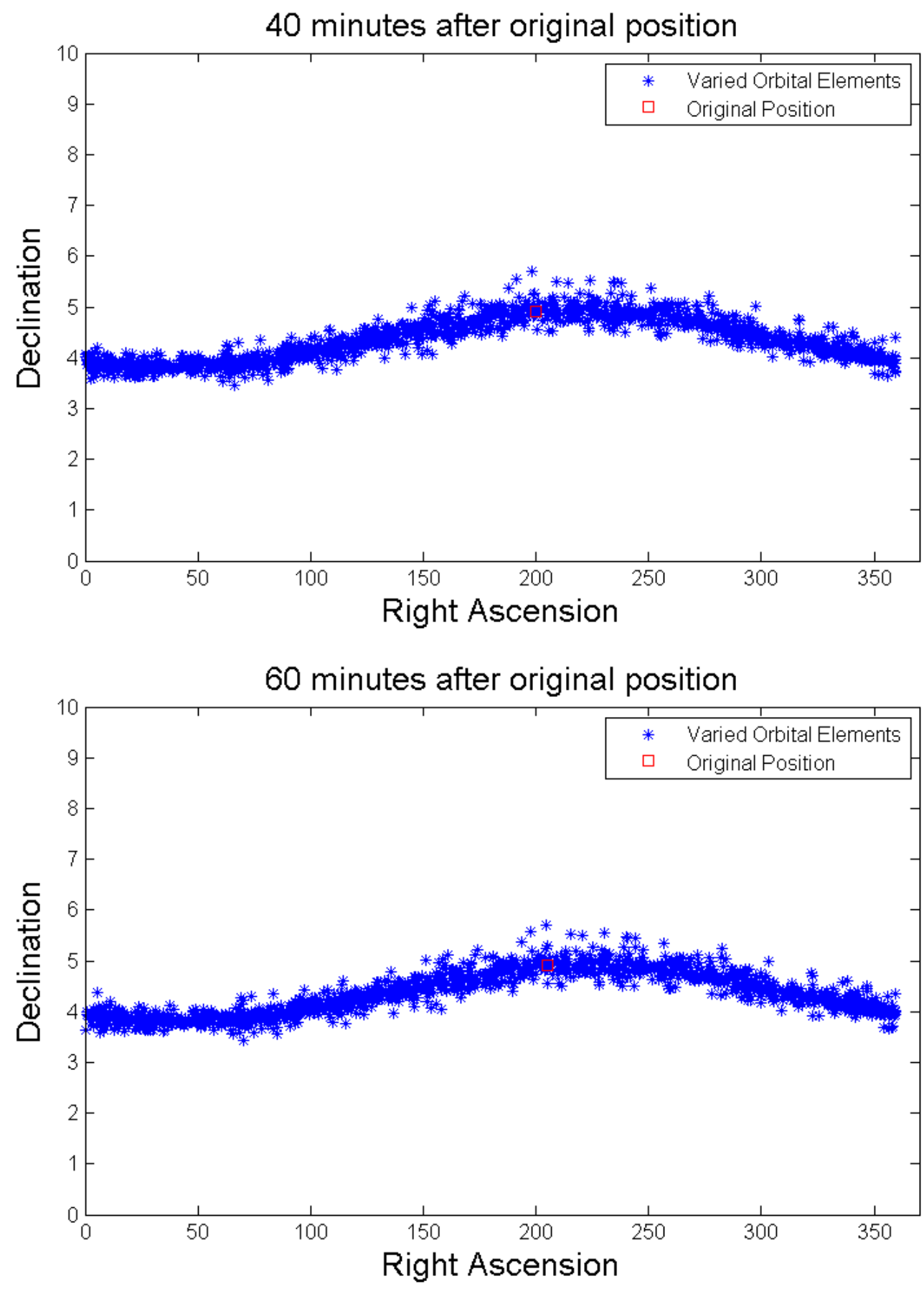


\section{Object 70702}

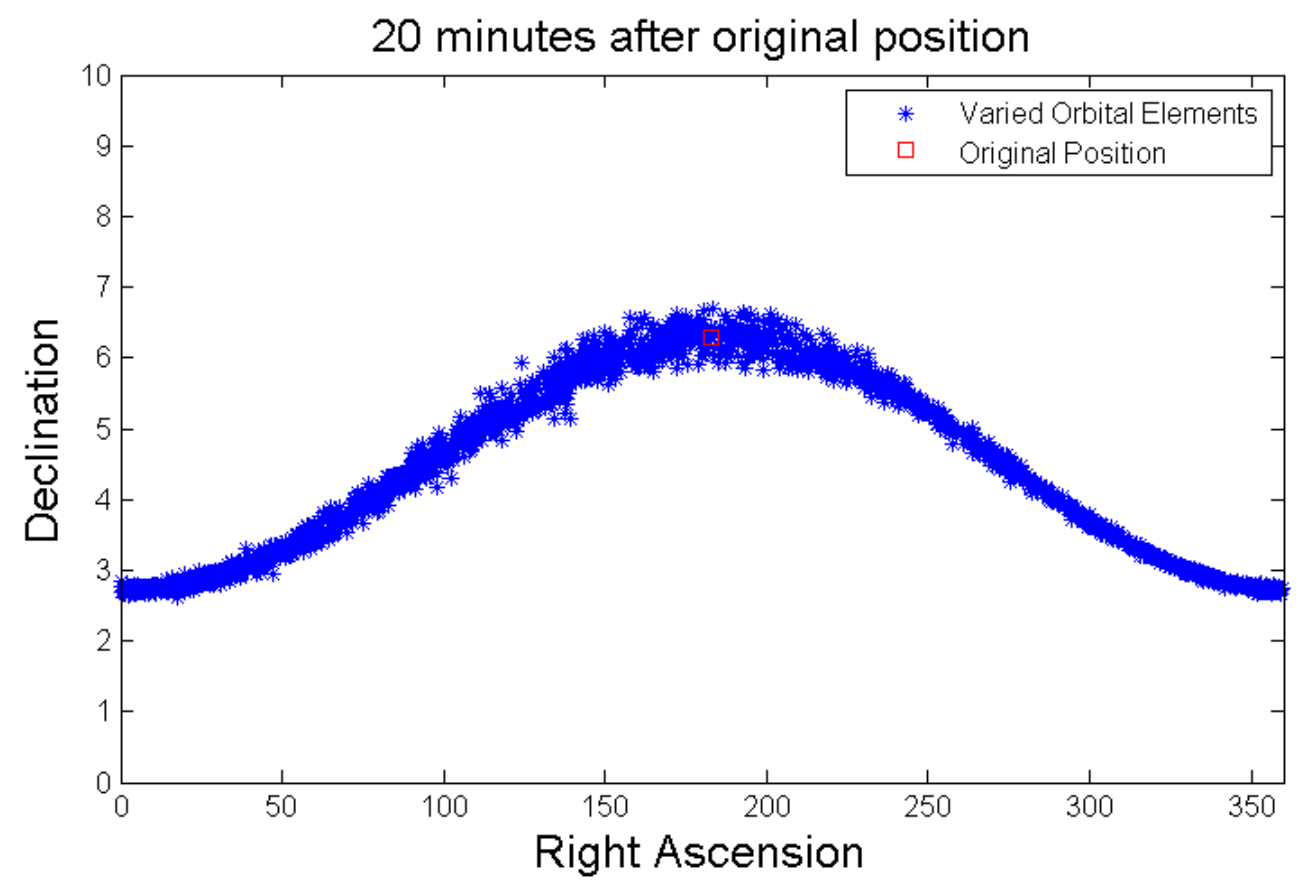



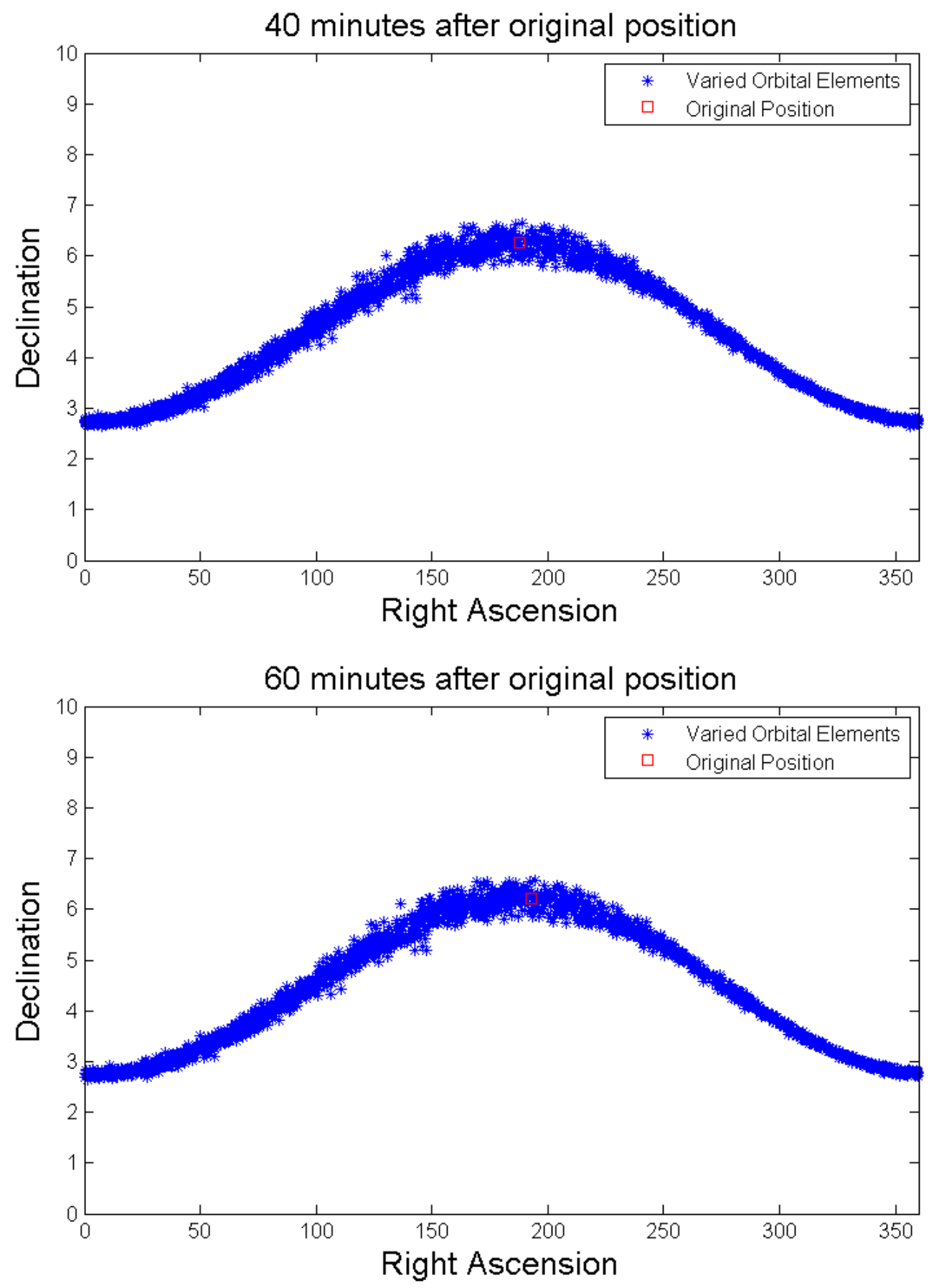


\section{Object 70703}

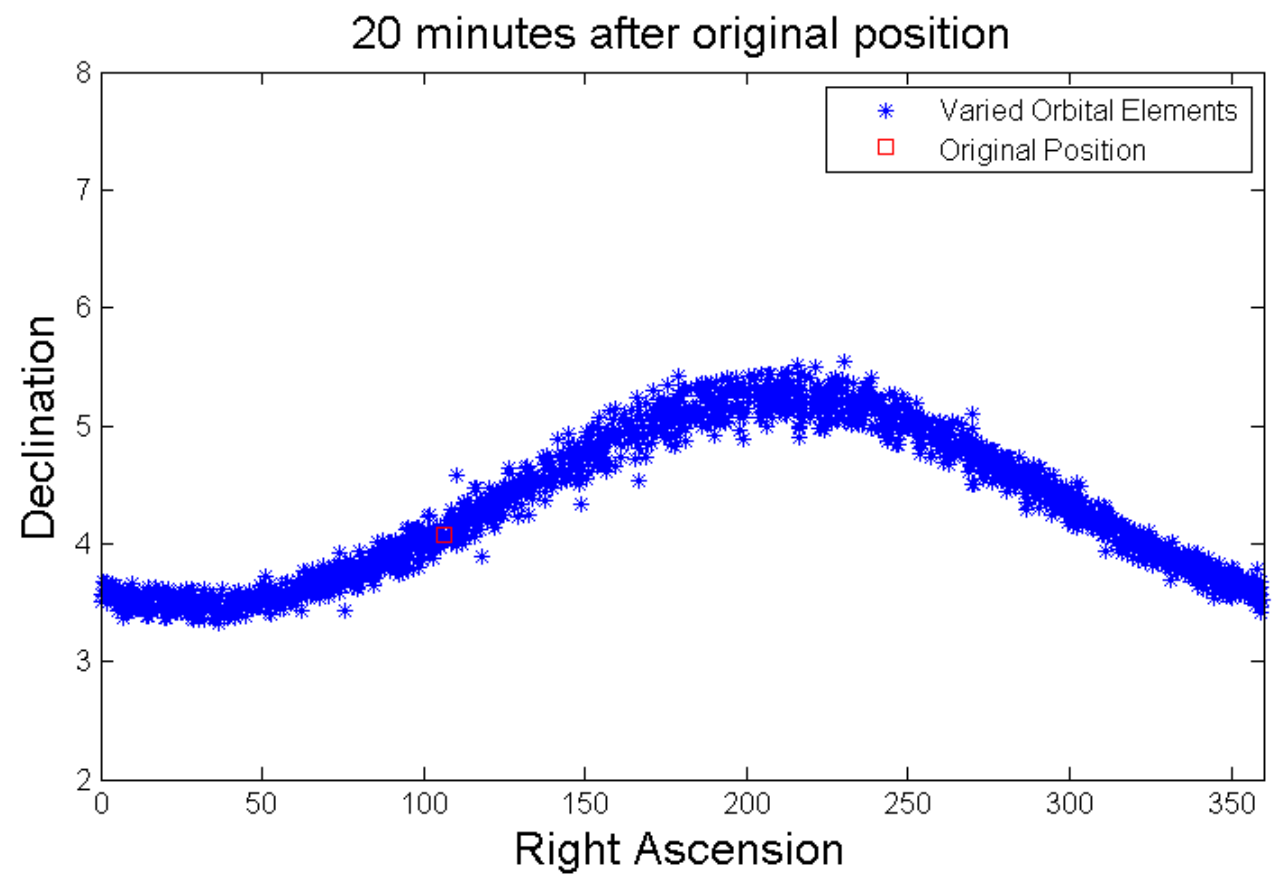



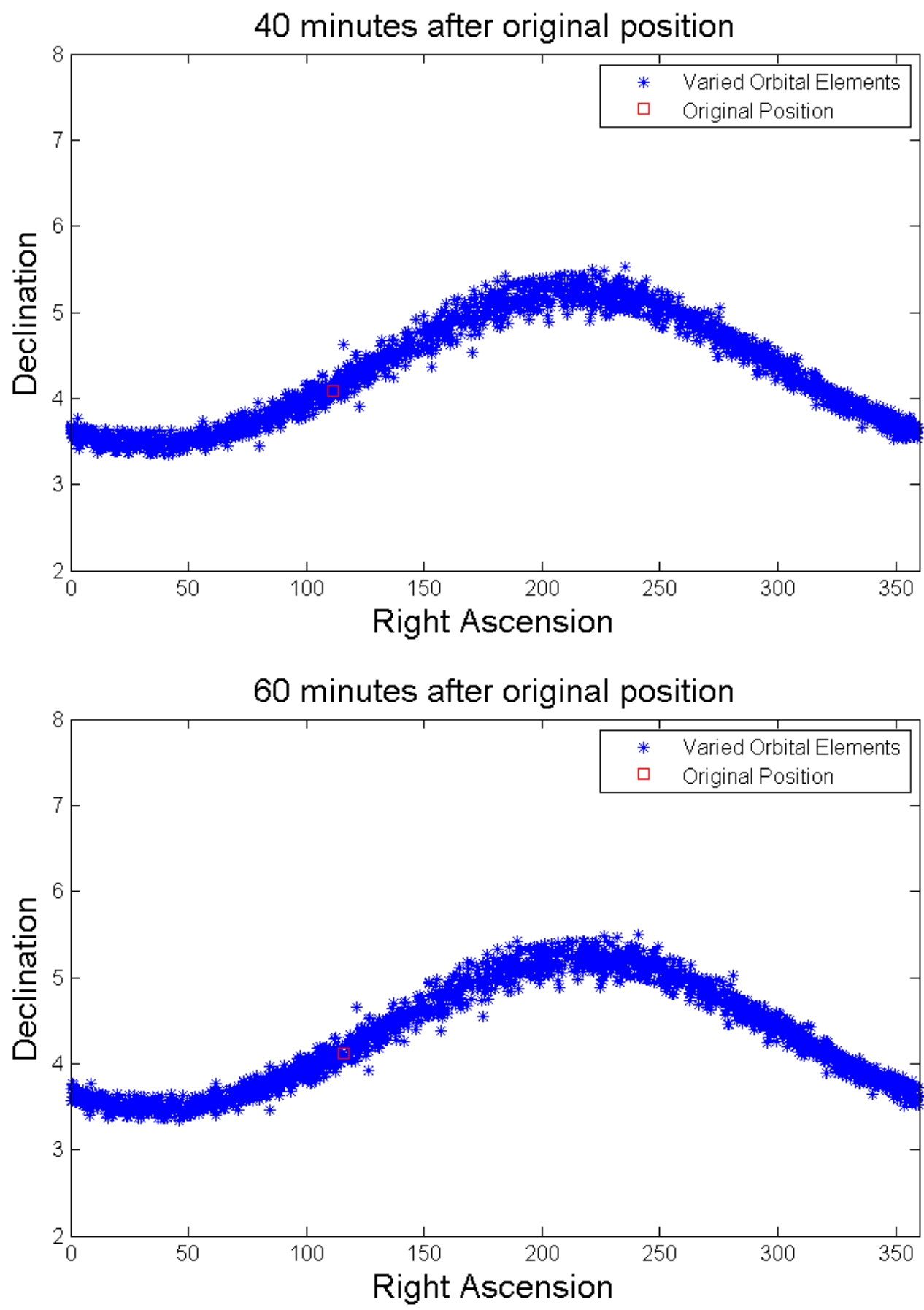


\section{Object 70704}

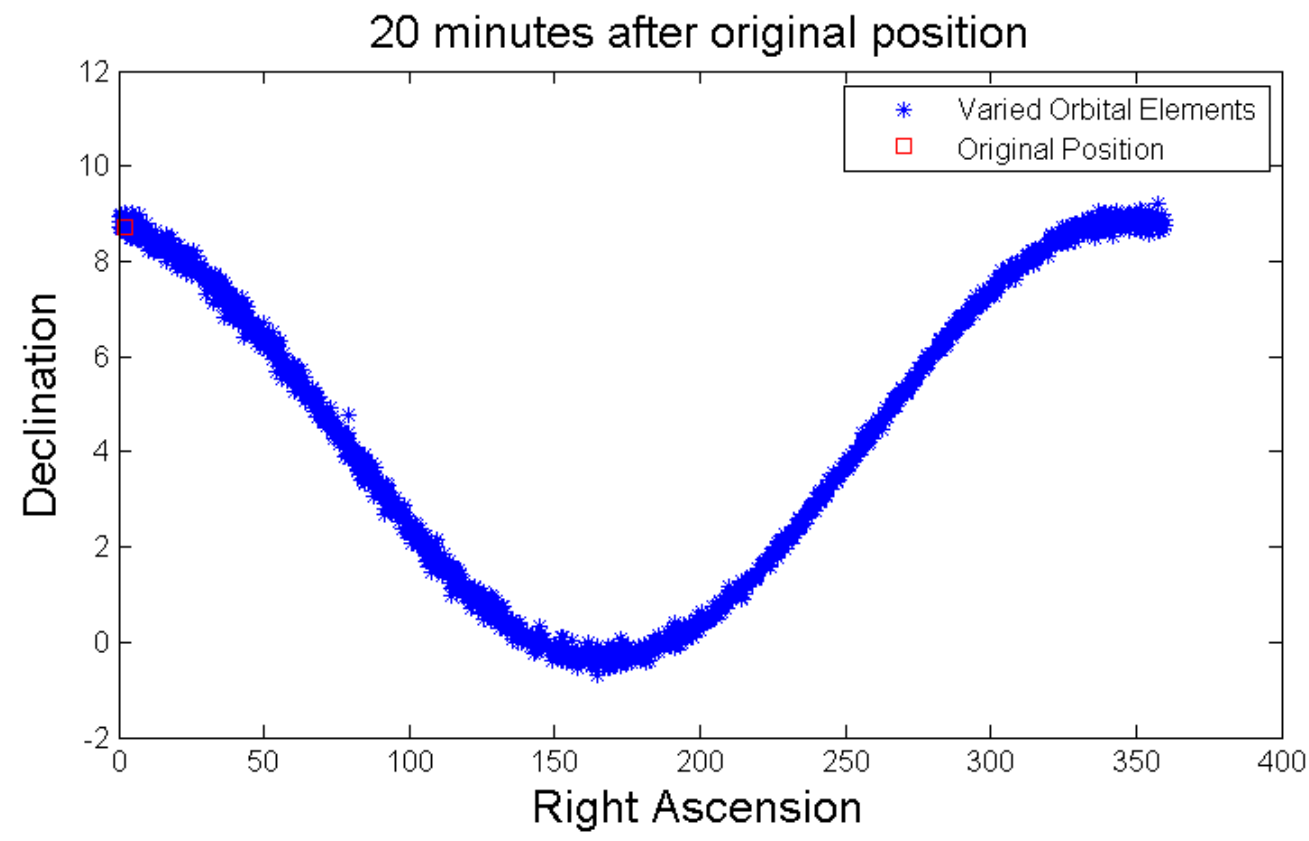



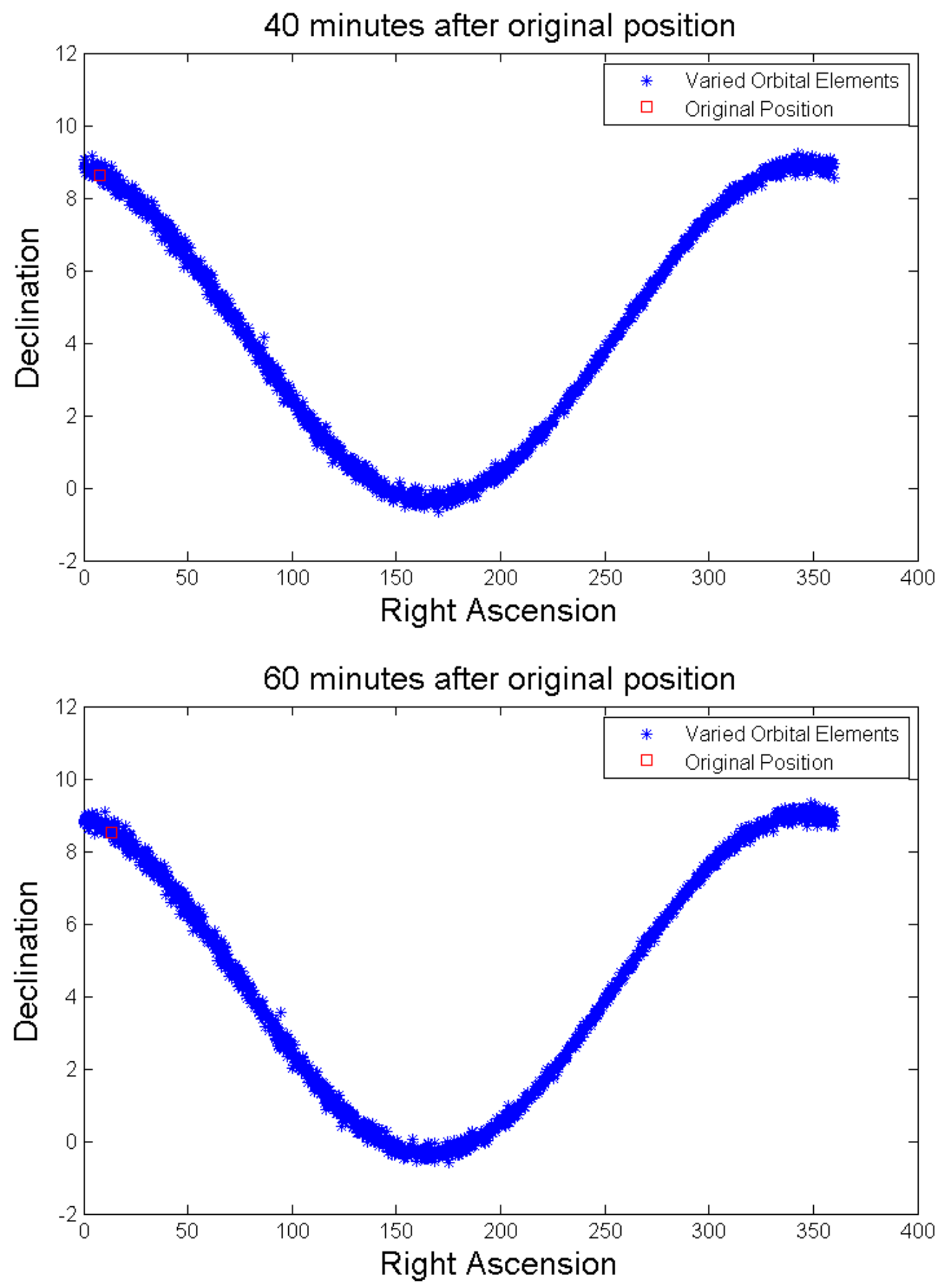


\section{Object 70705}

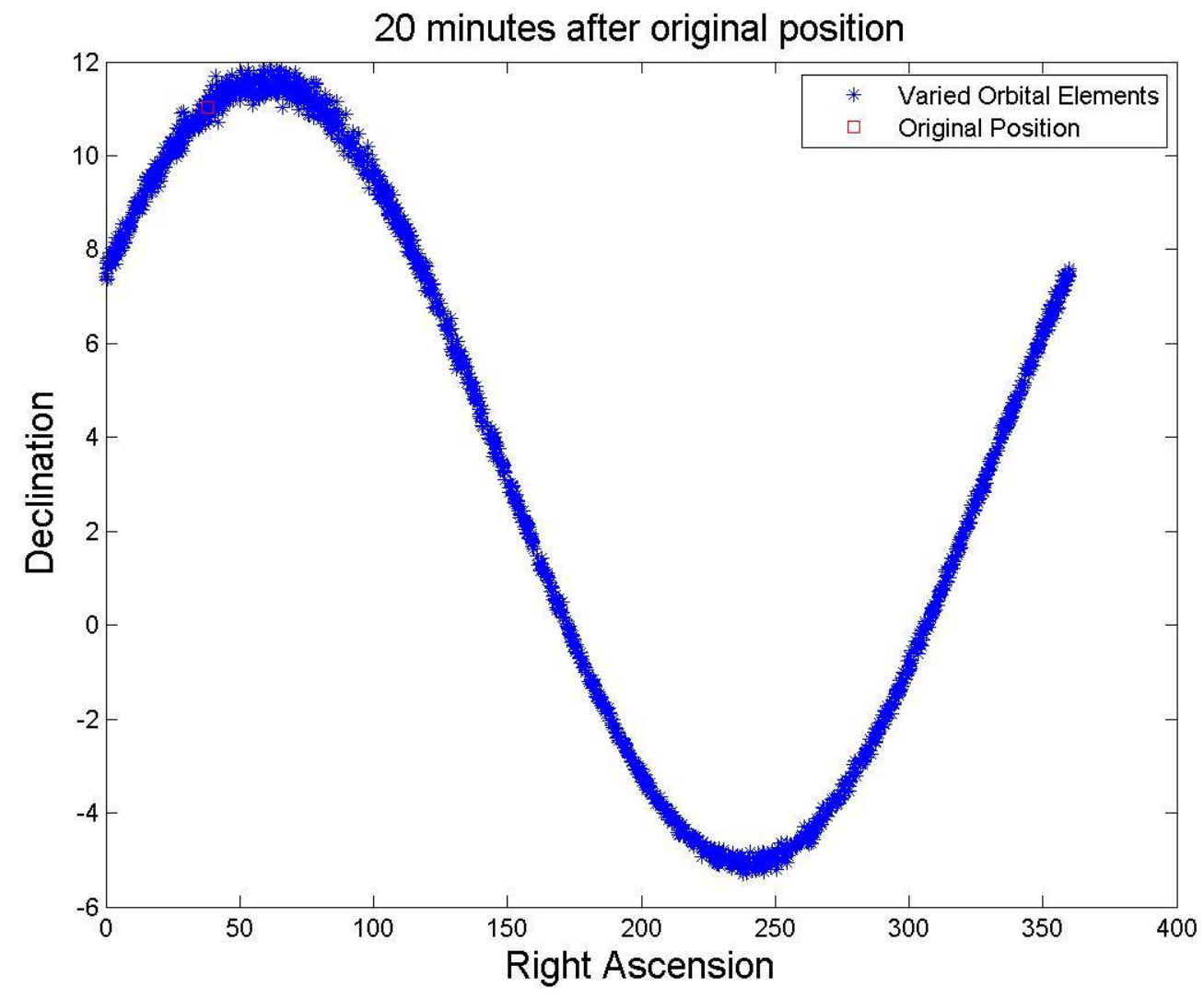



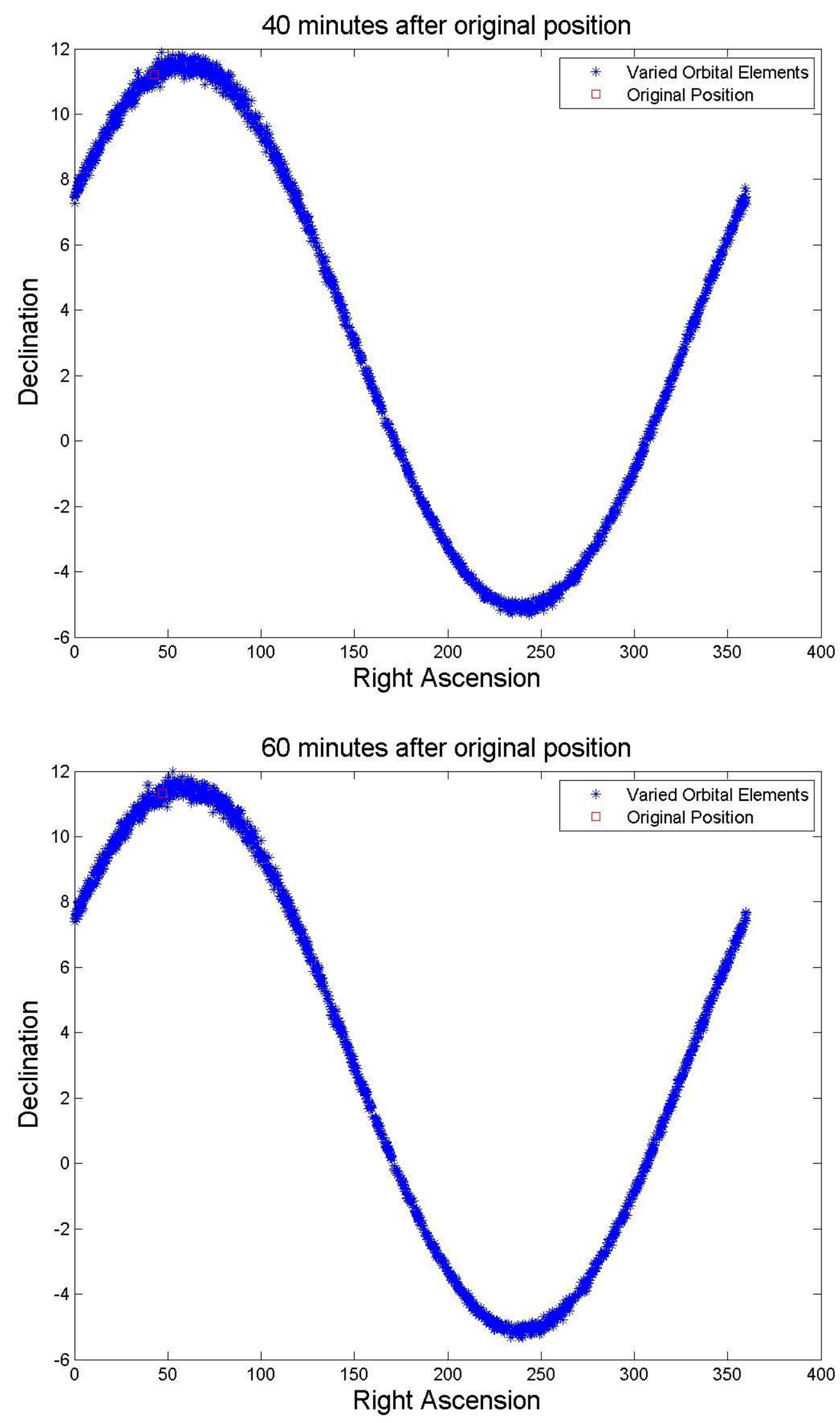


\section{Object 70706}

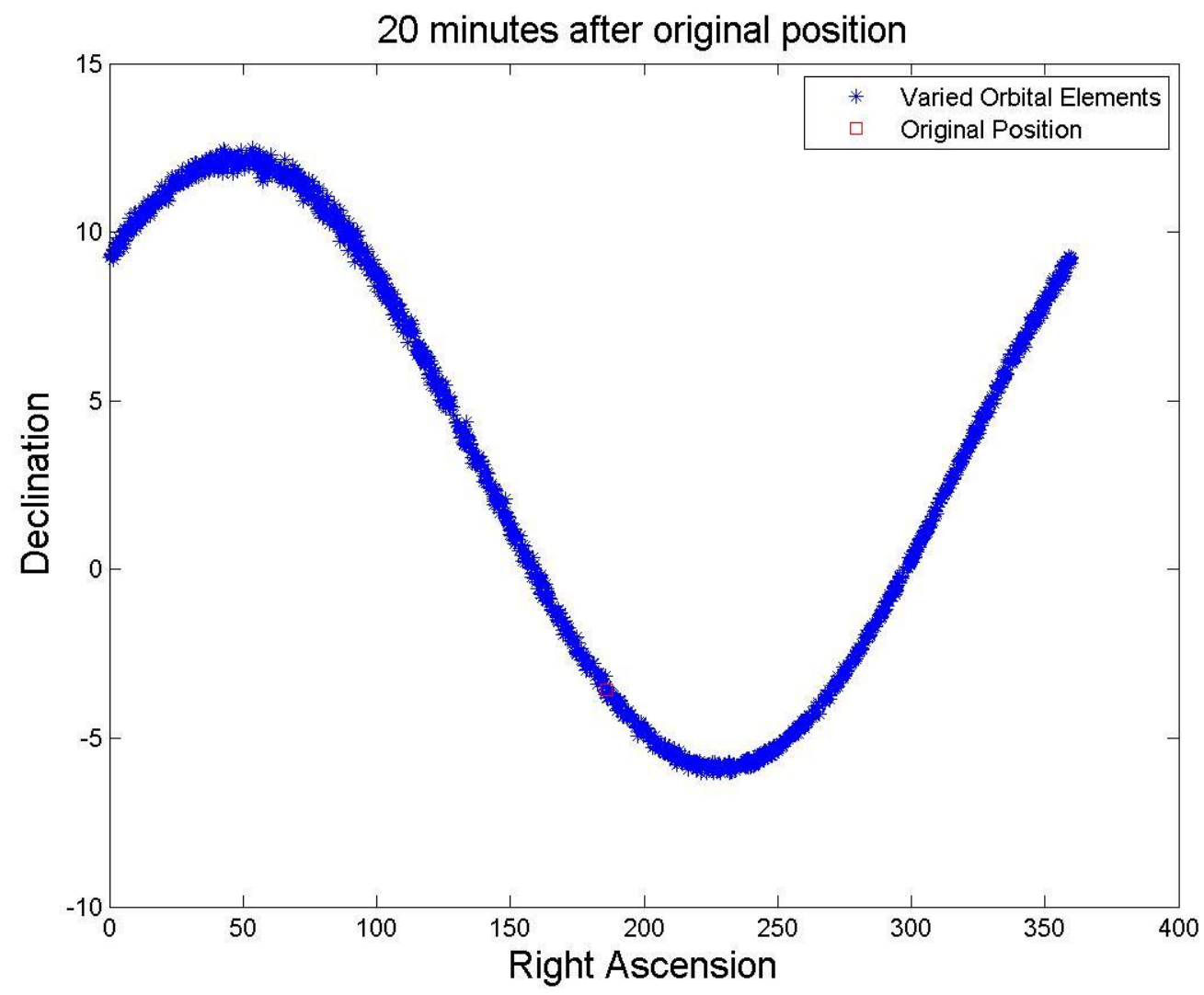



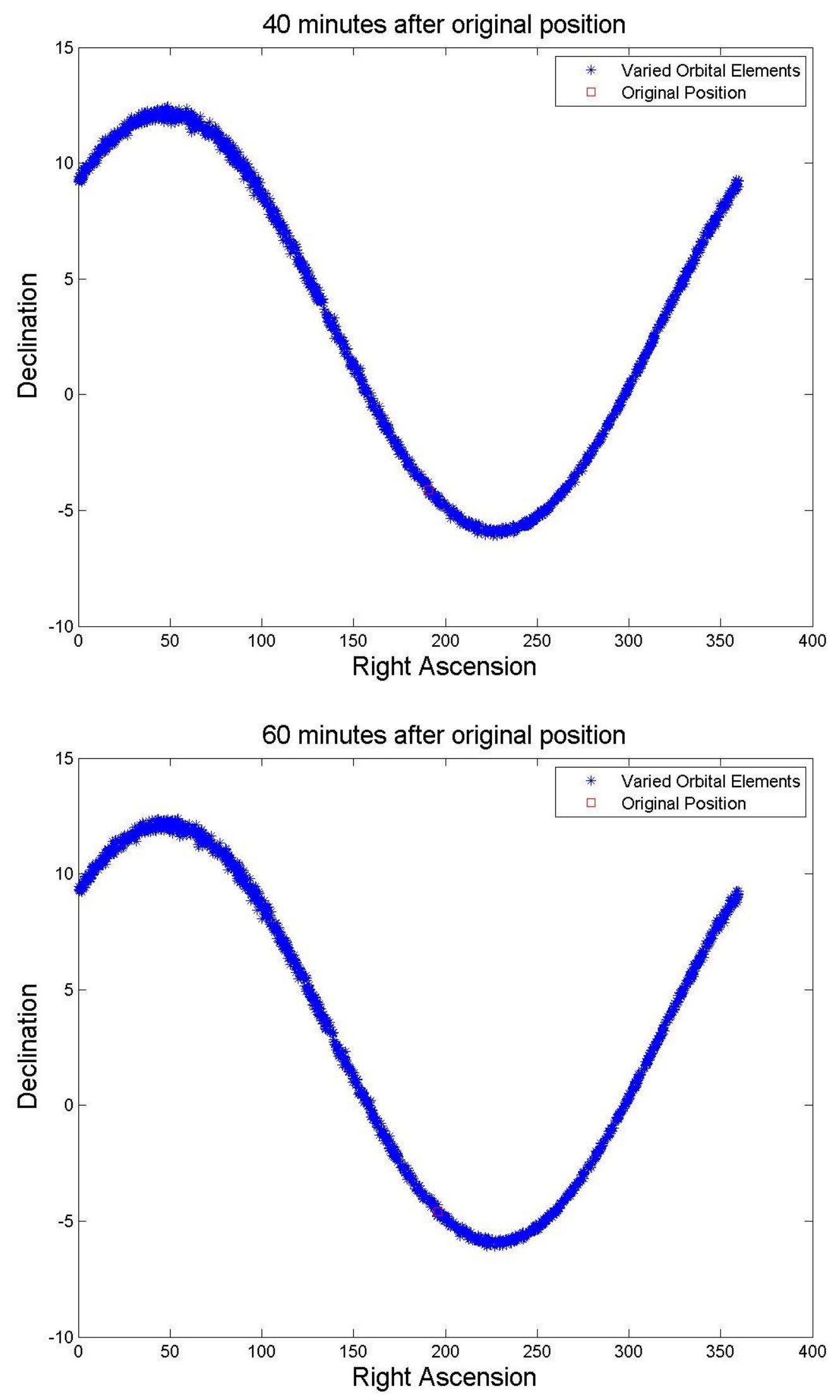


\section{Object 70707}

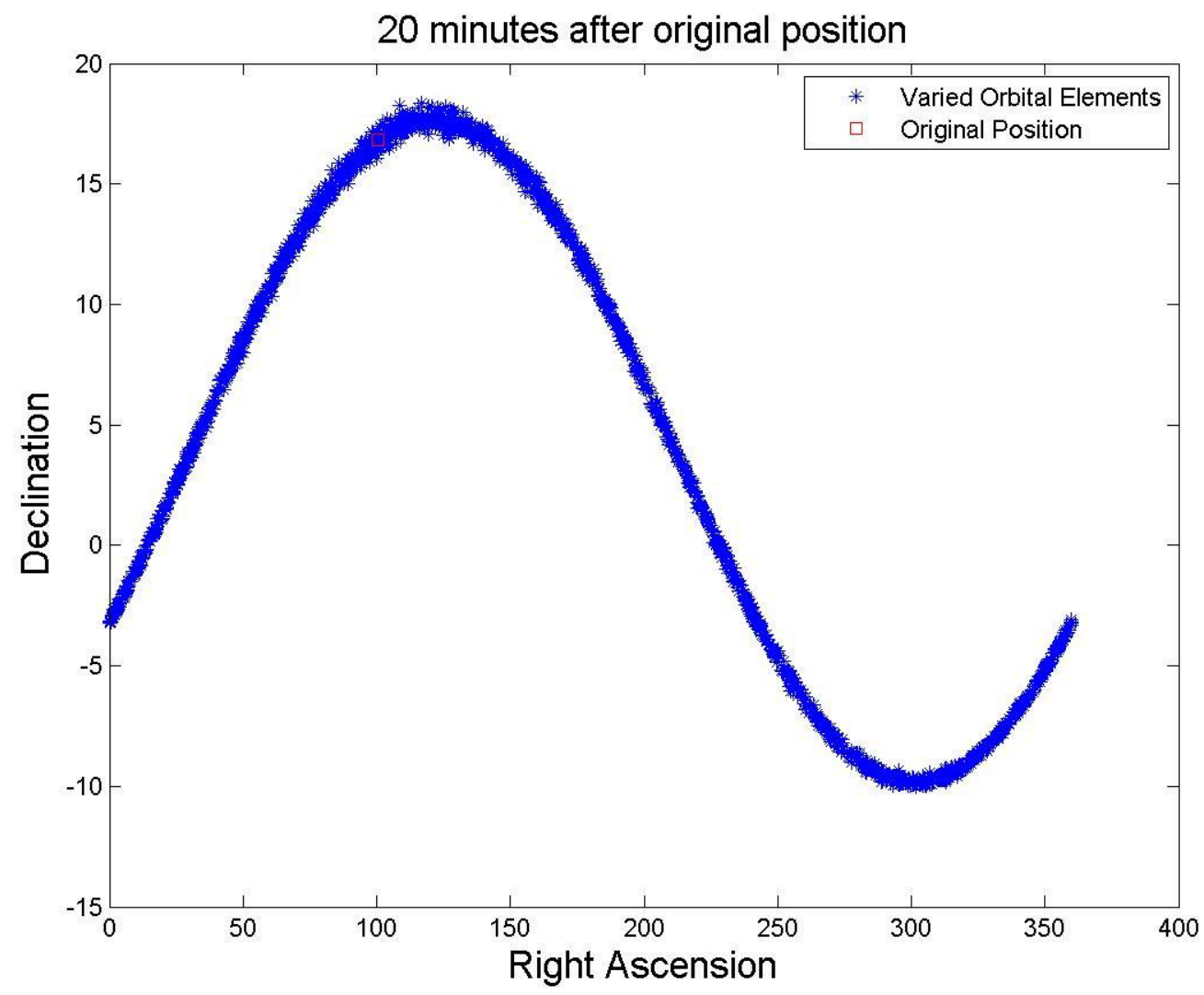



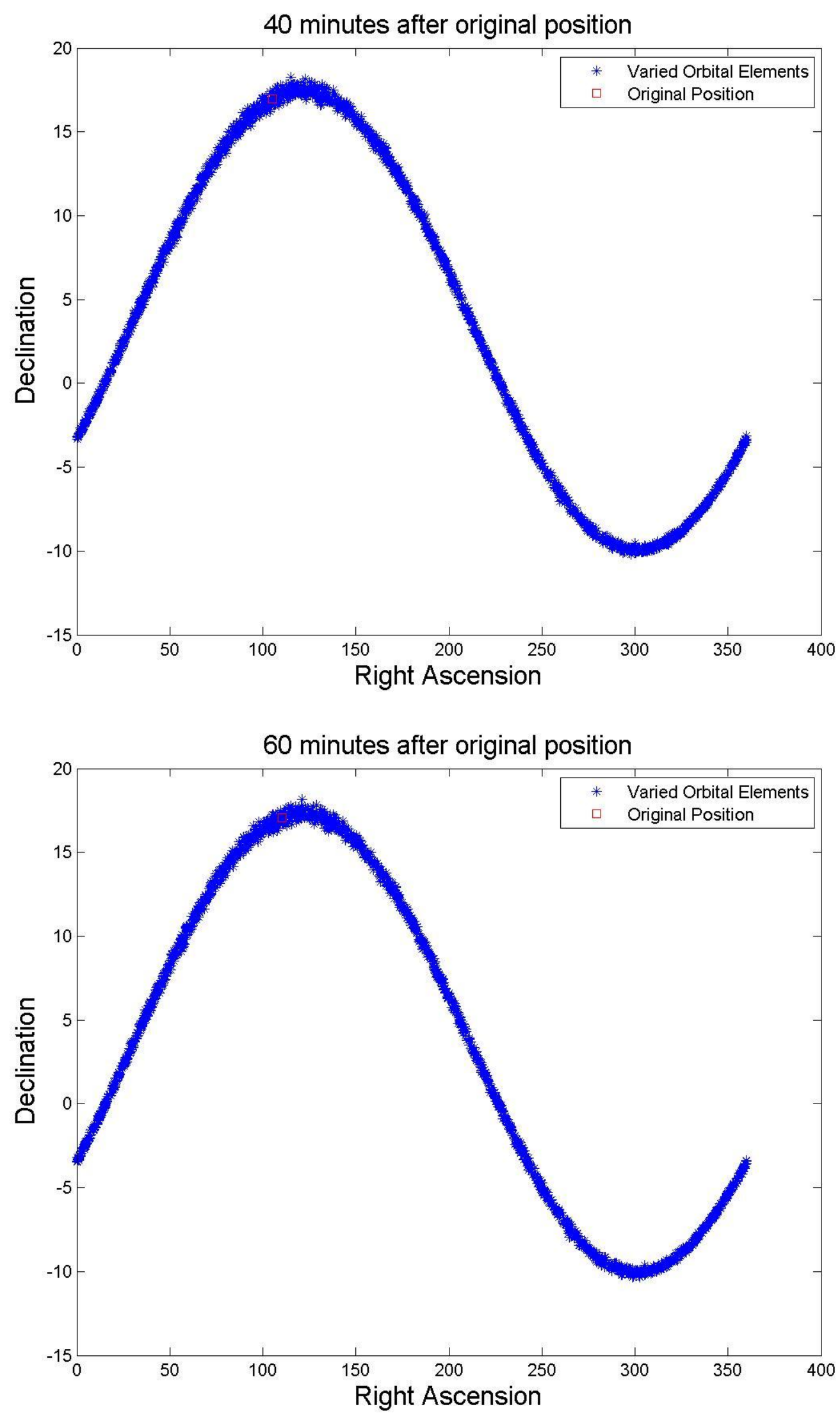


\section{Object 70708}

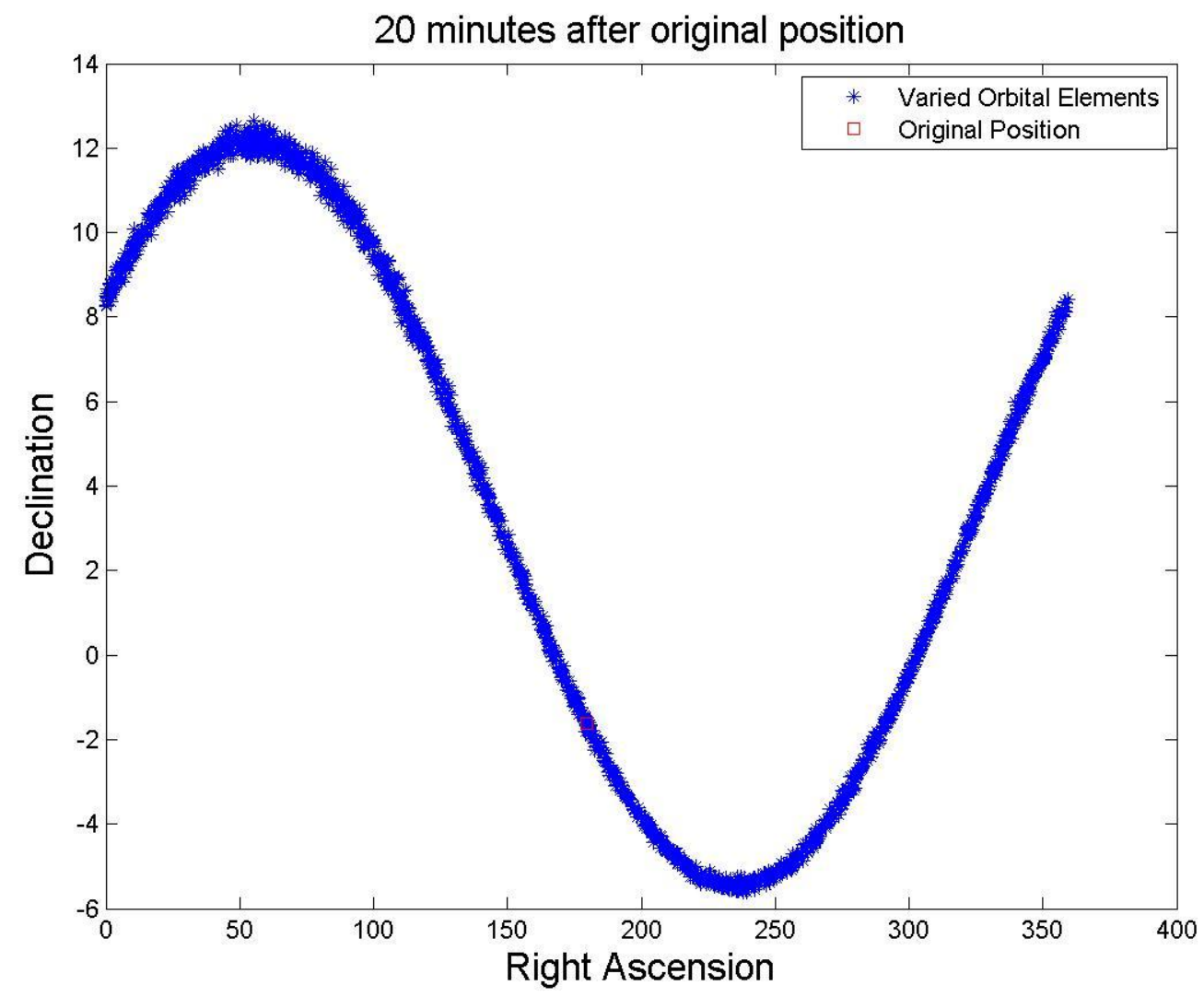



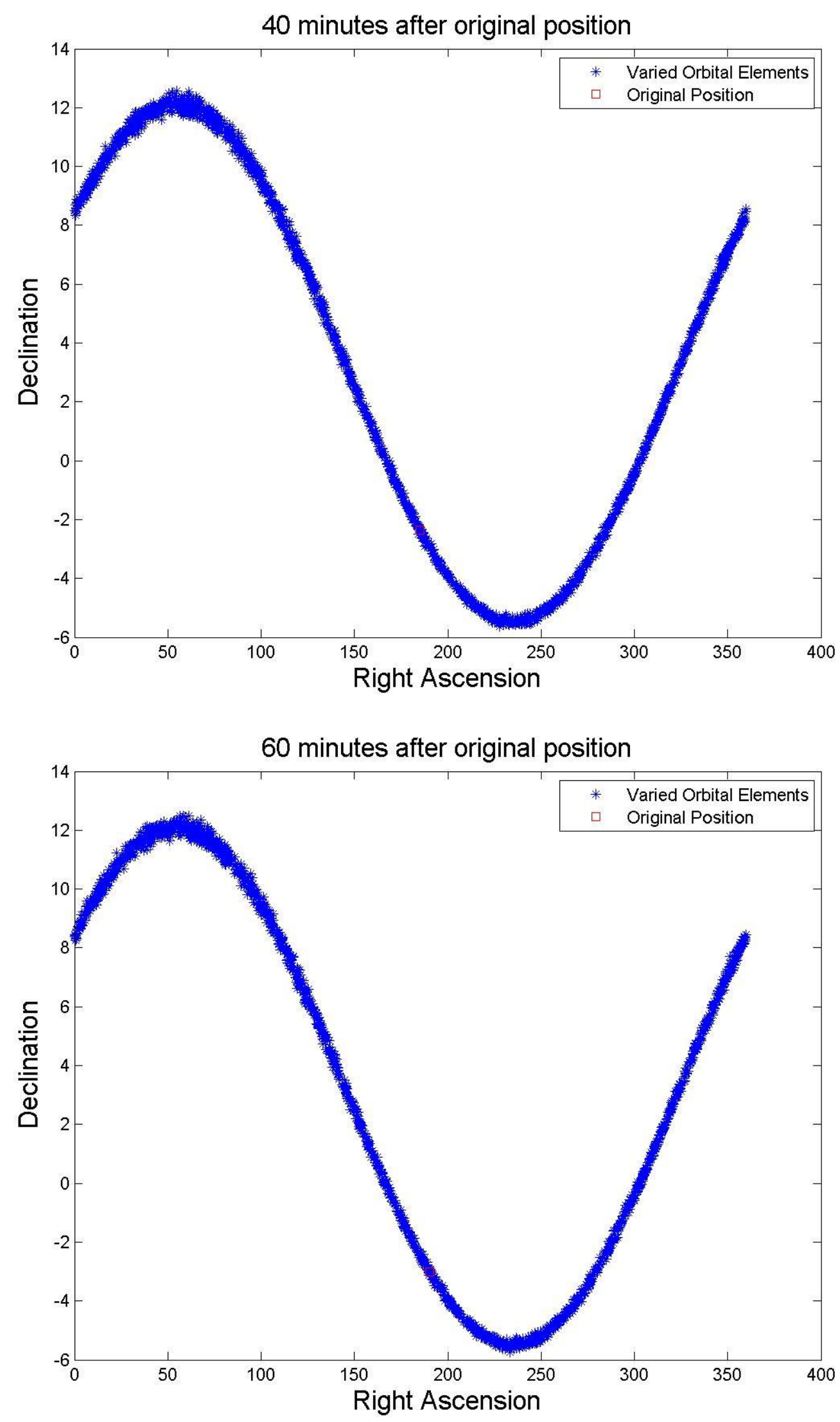


\section{Object 70709}

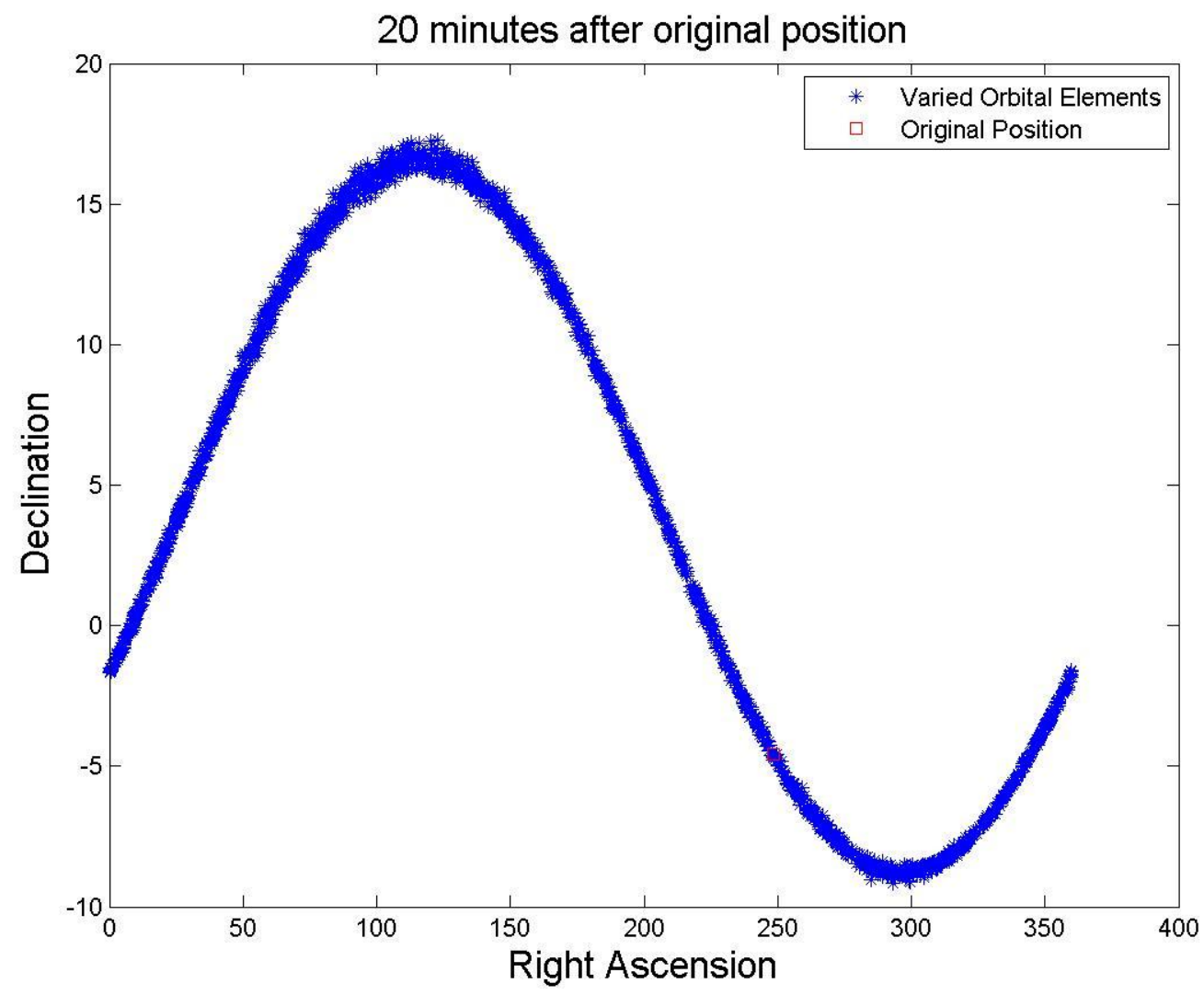



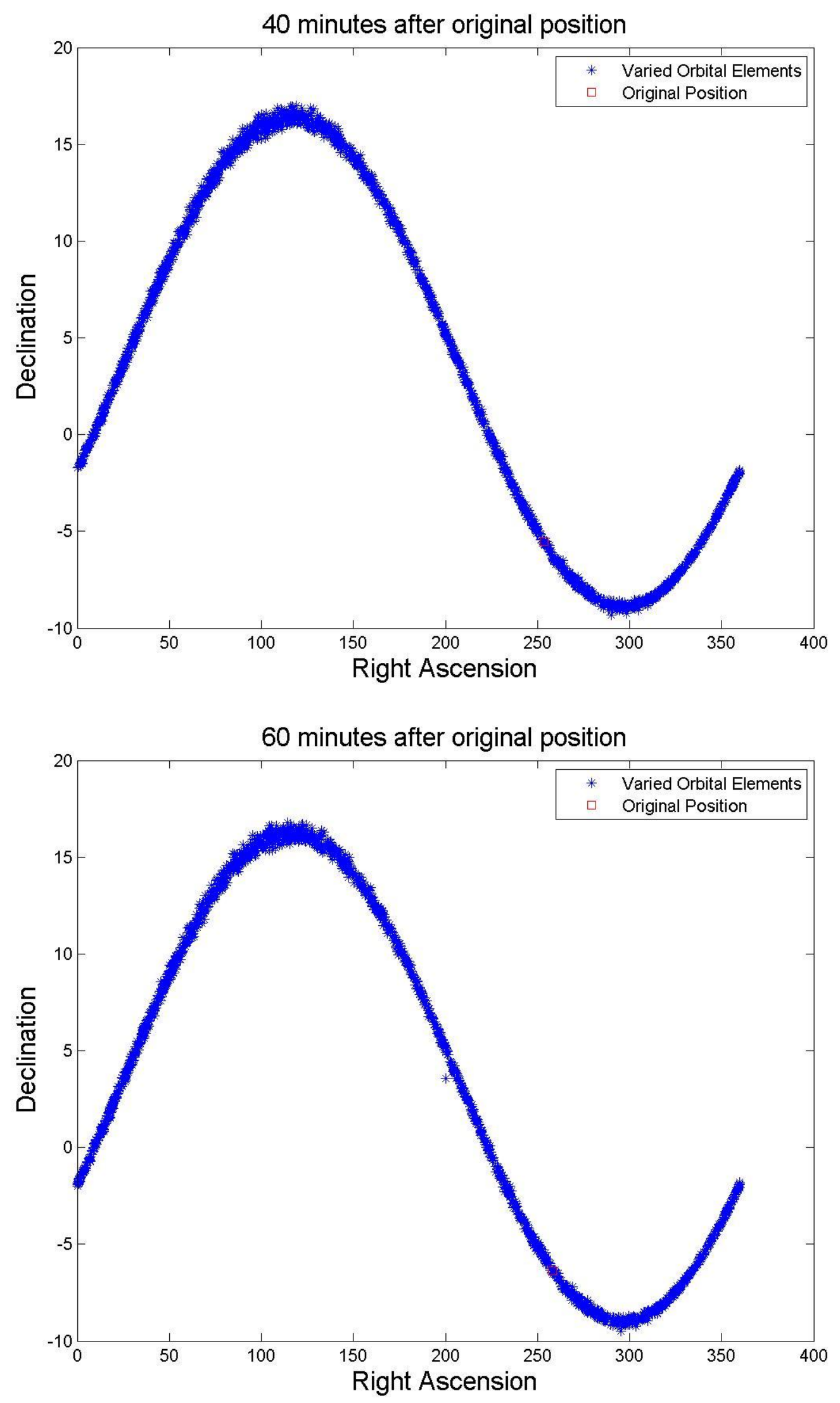


\section{Object 70710}

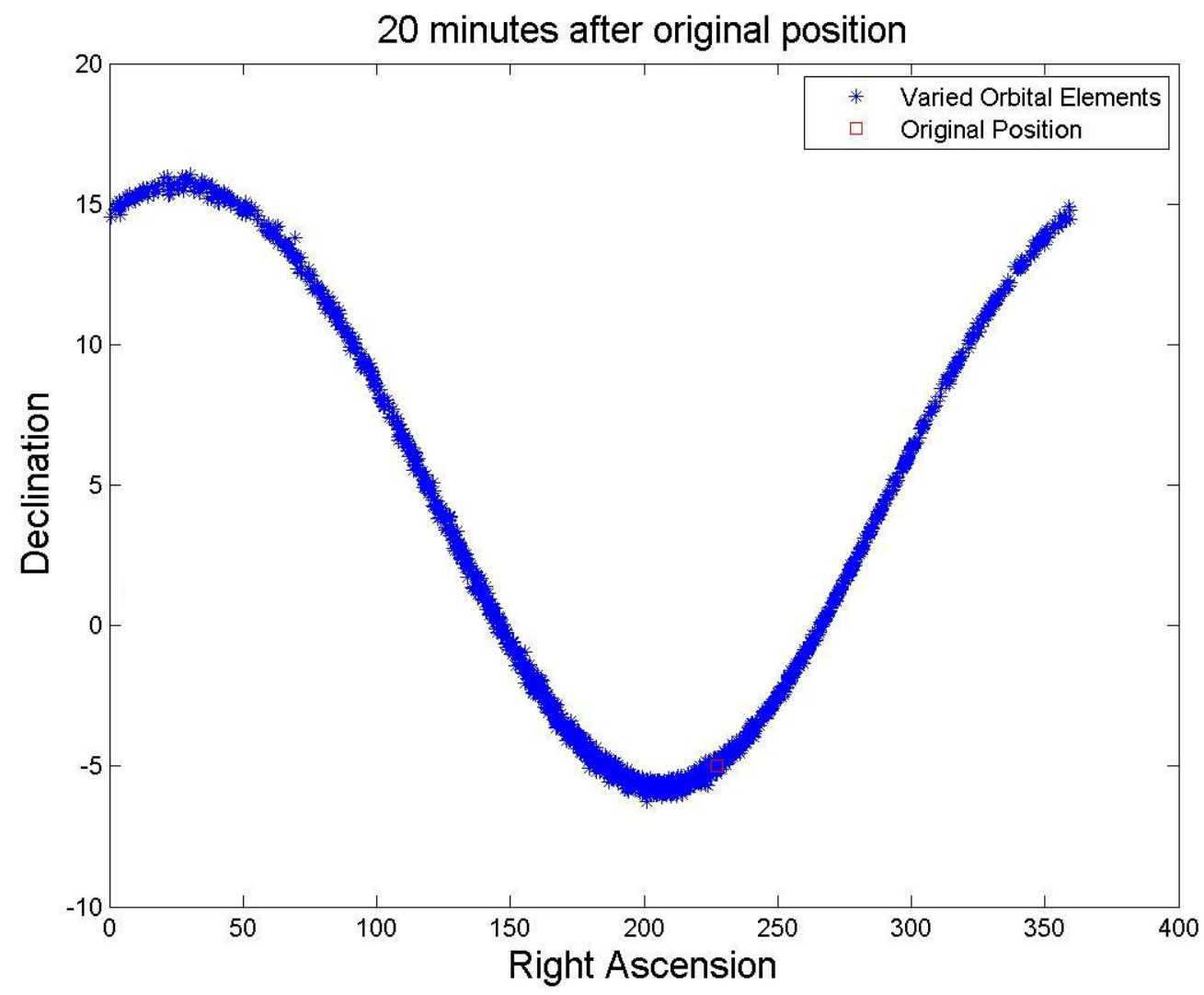



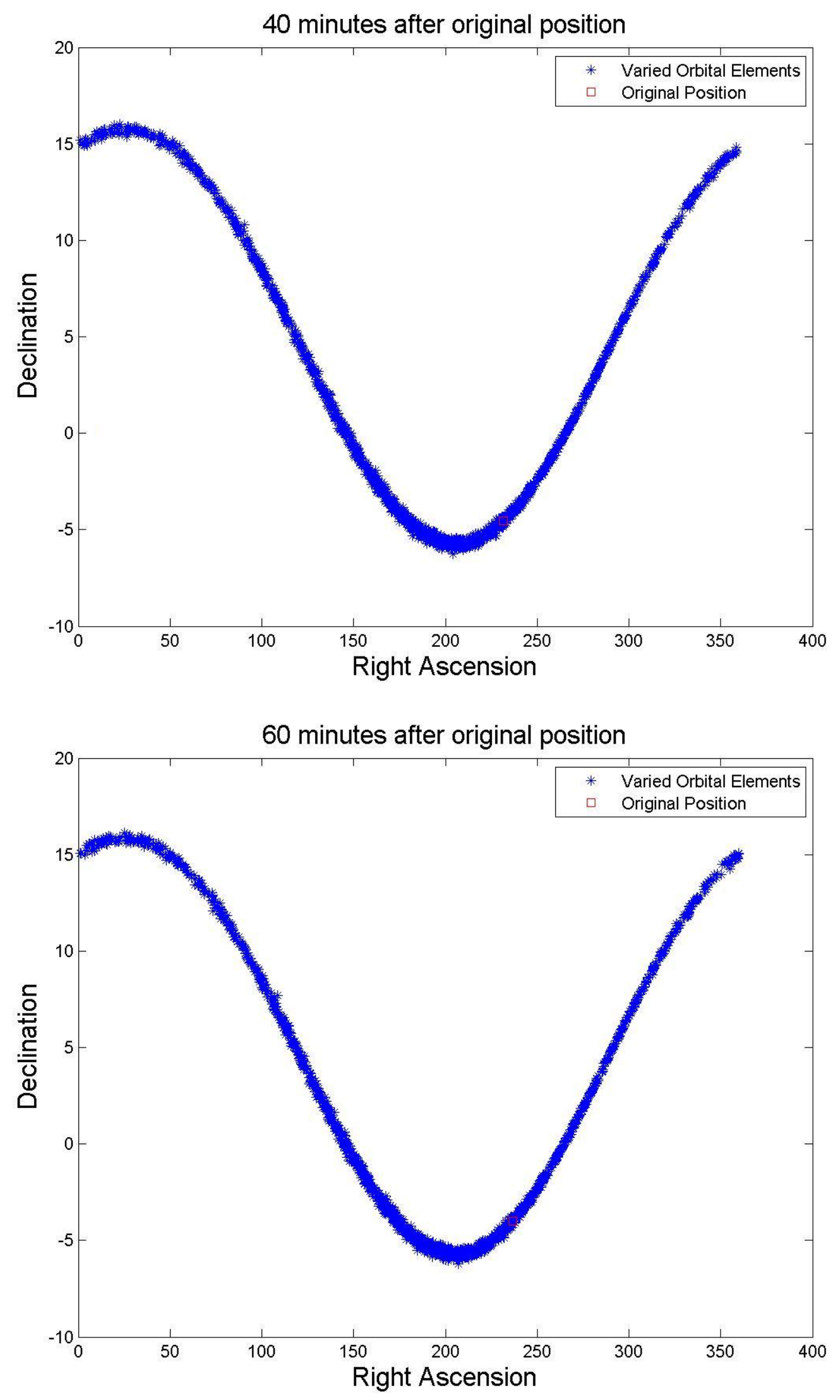


\section{Object 70711}

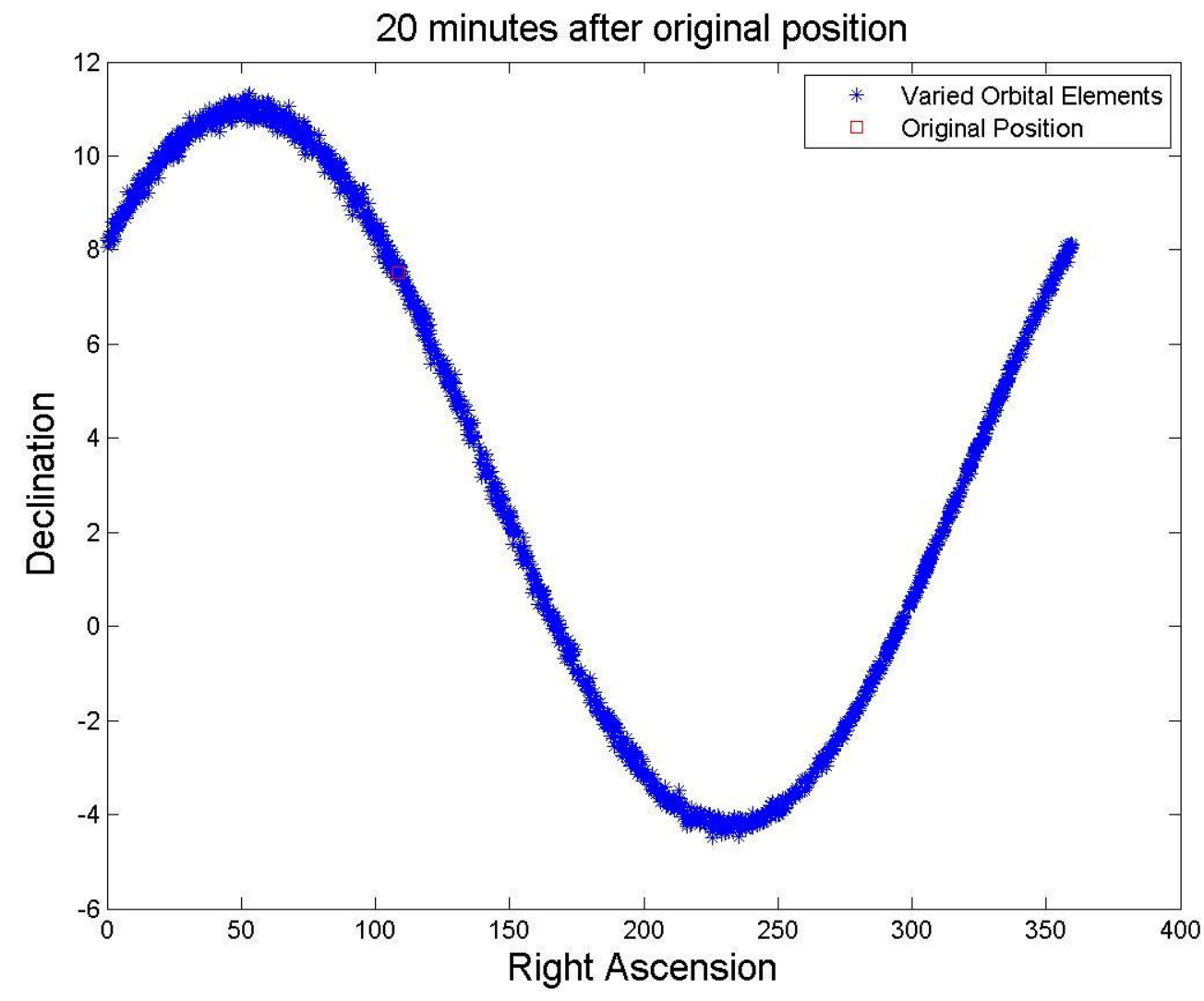



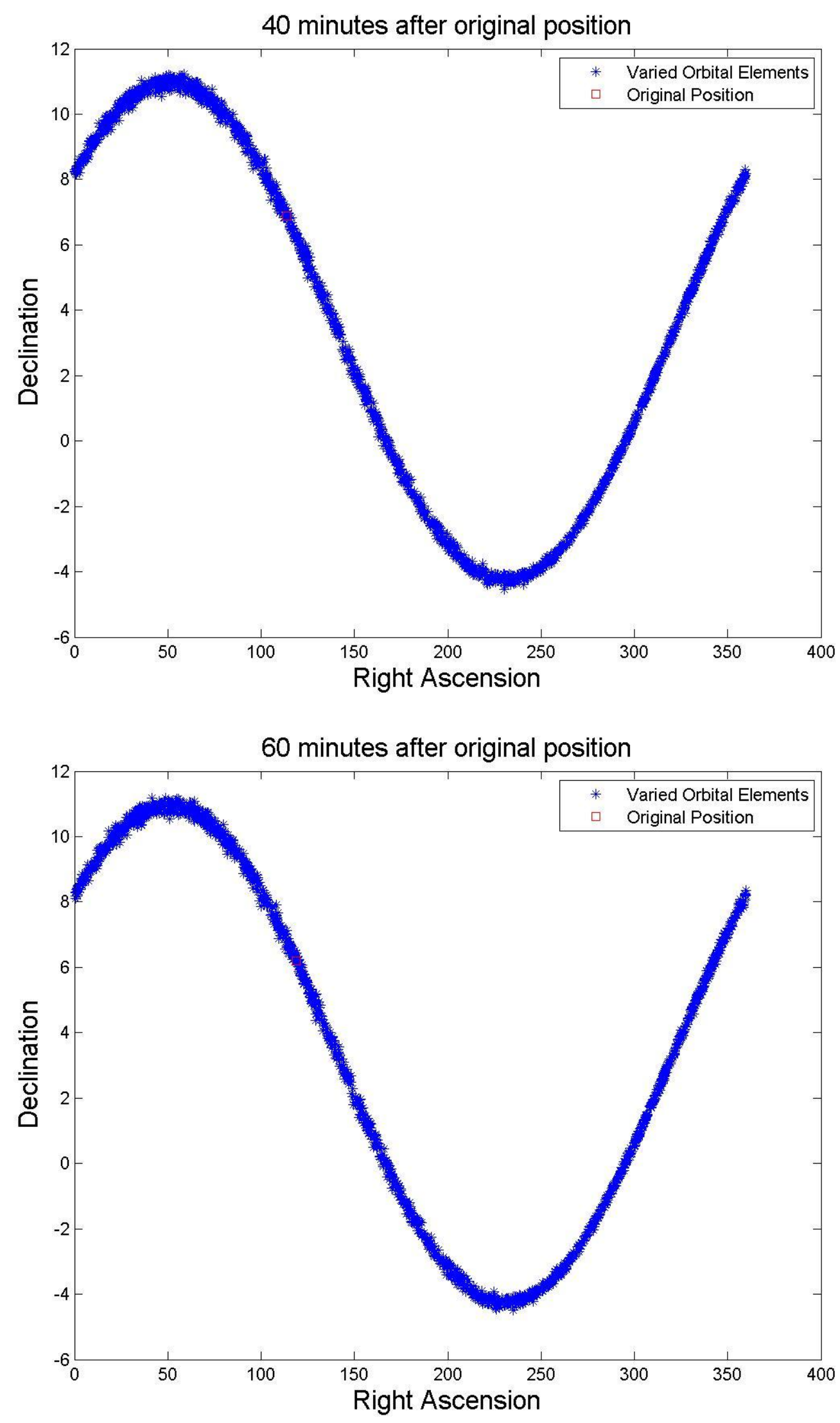


\section{Object 70712}

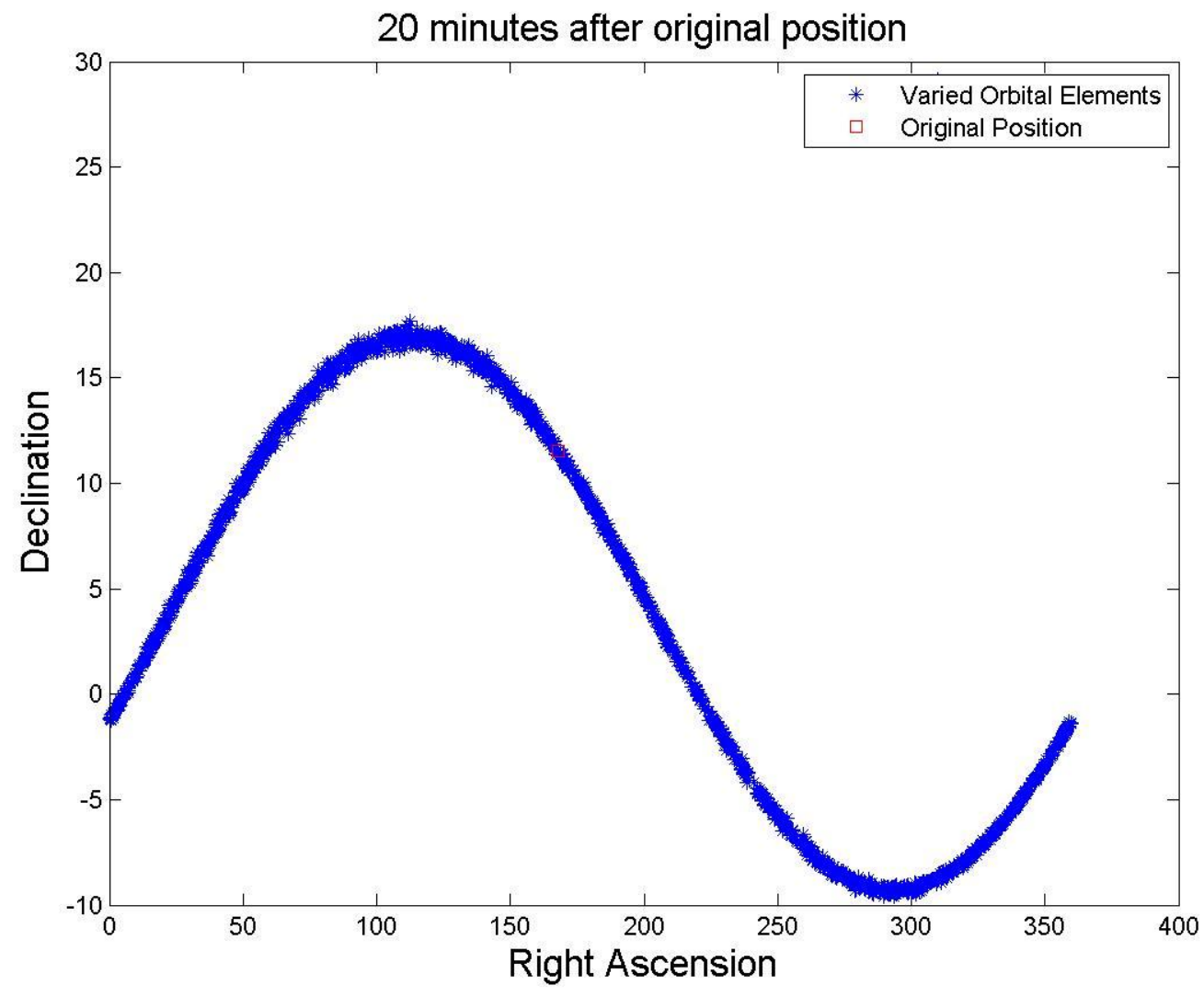



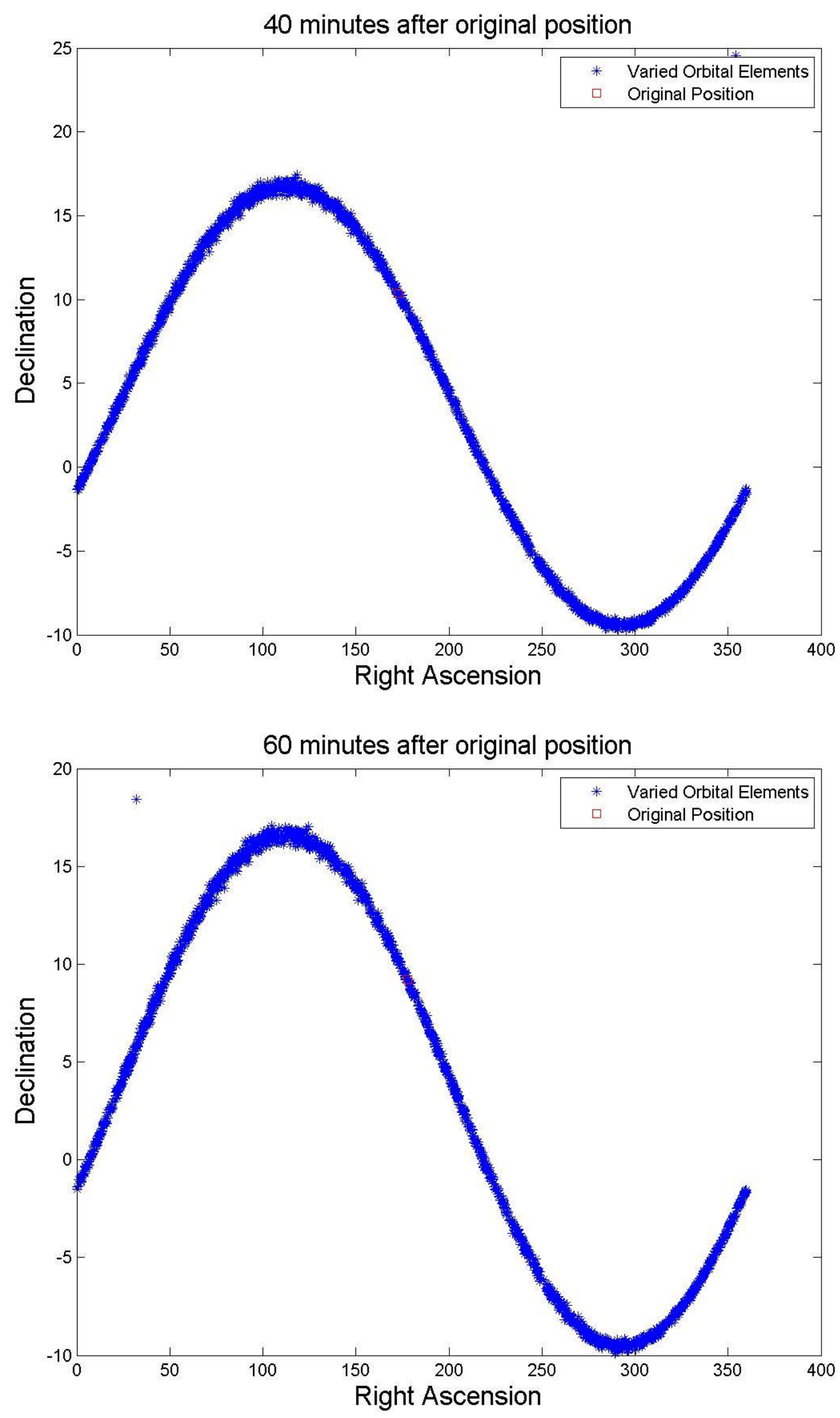


\section{Object 70713}

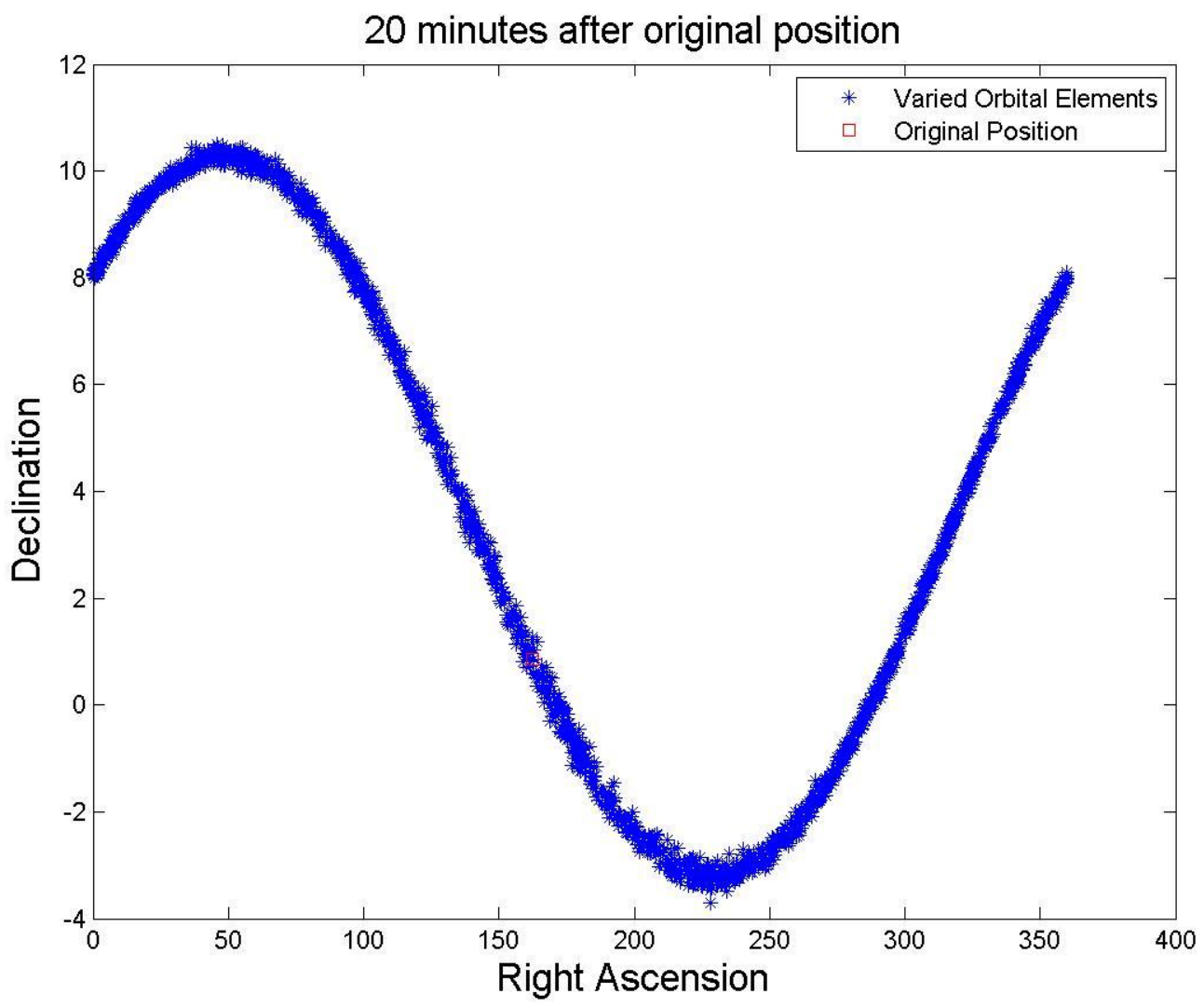



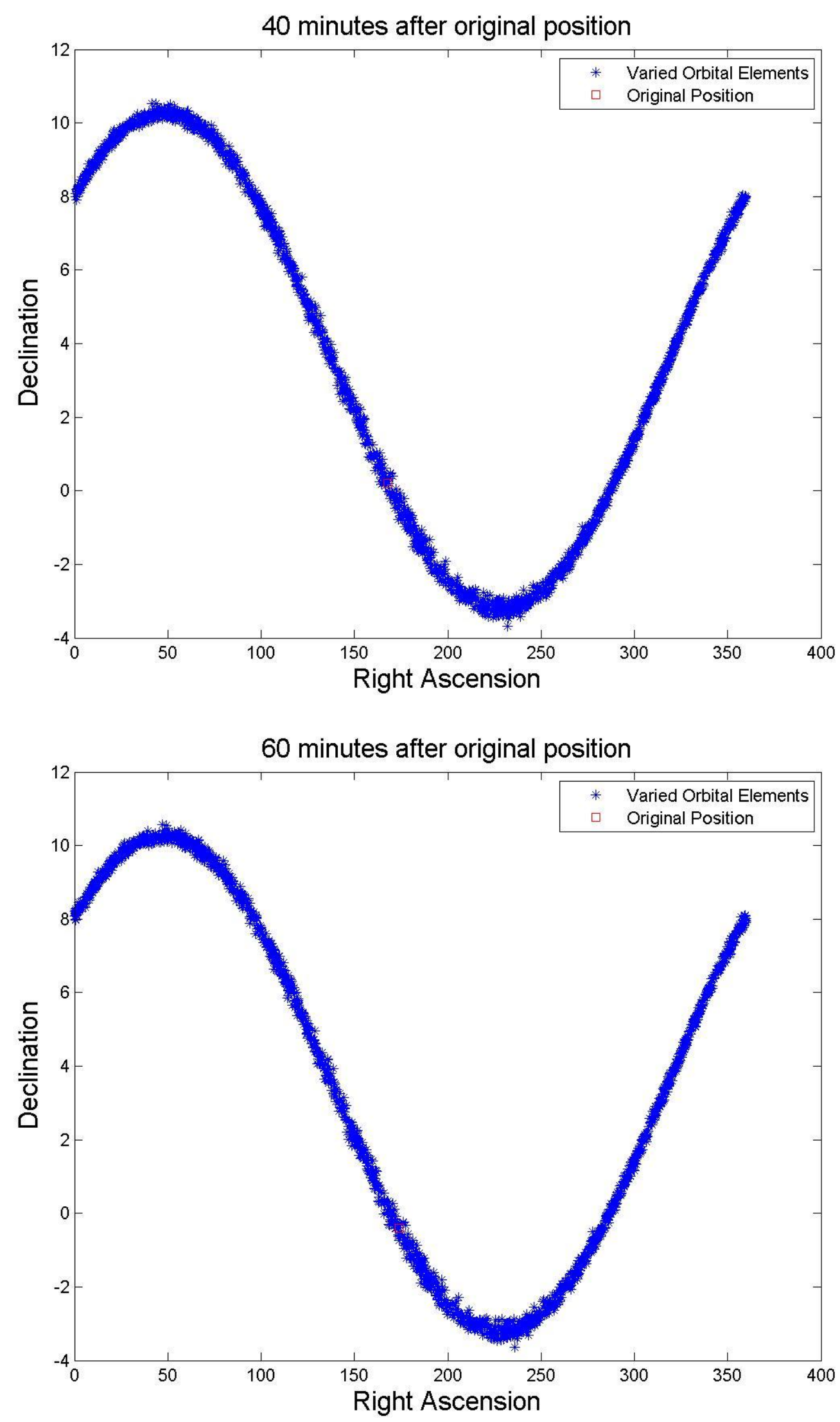
220

Object 70714

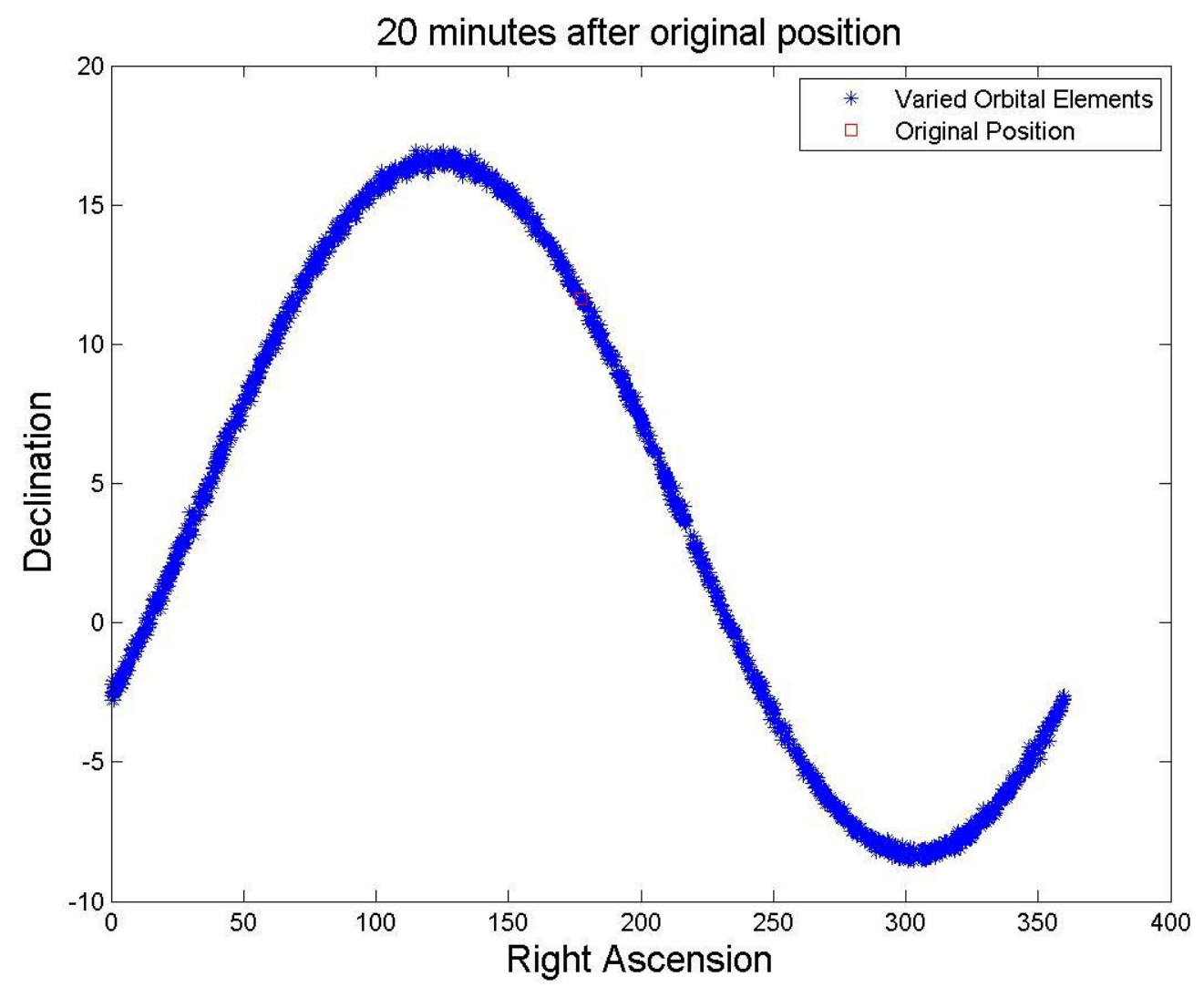



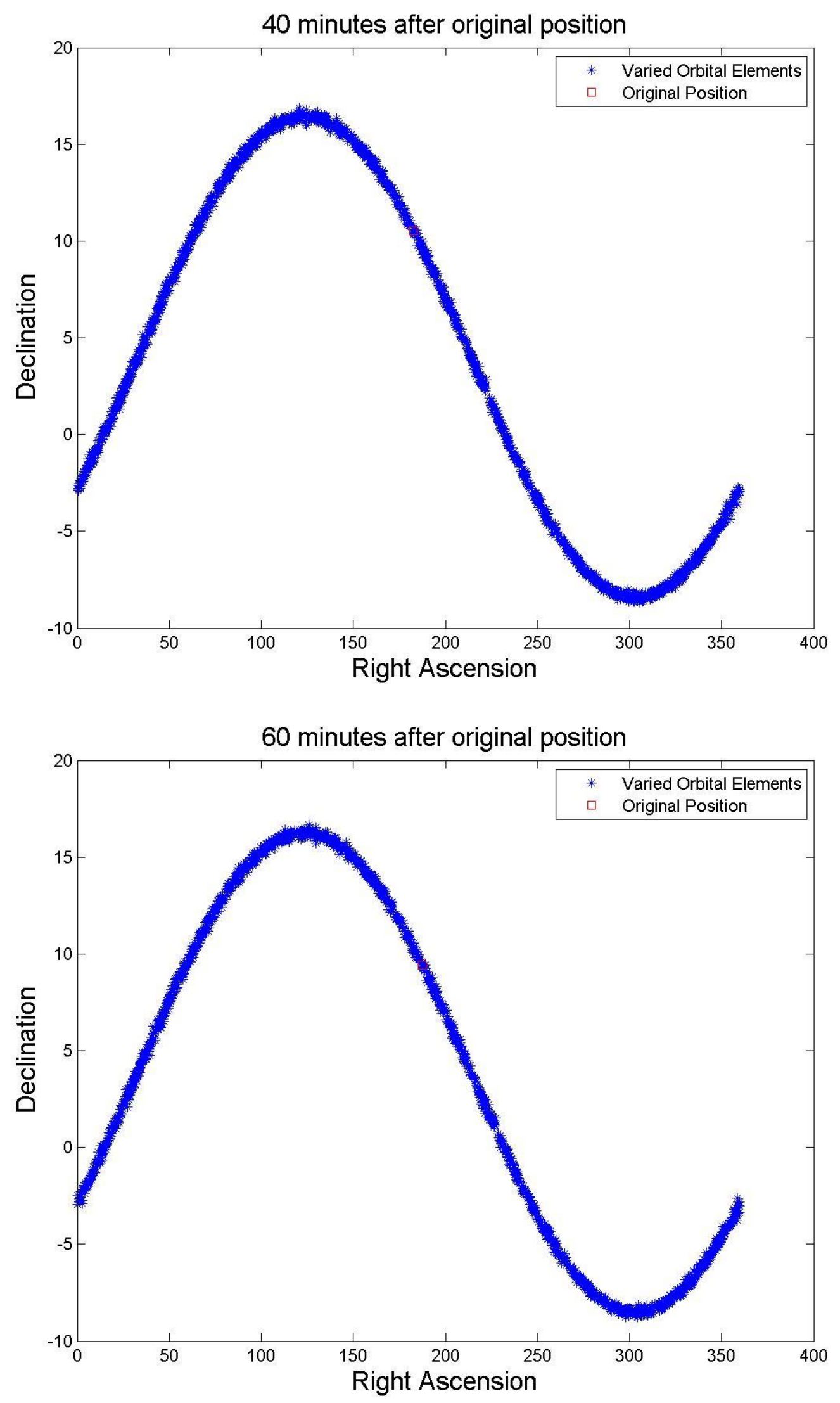


\section{Object 70715}

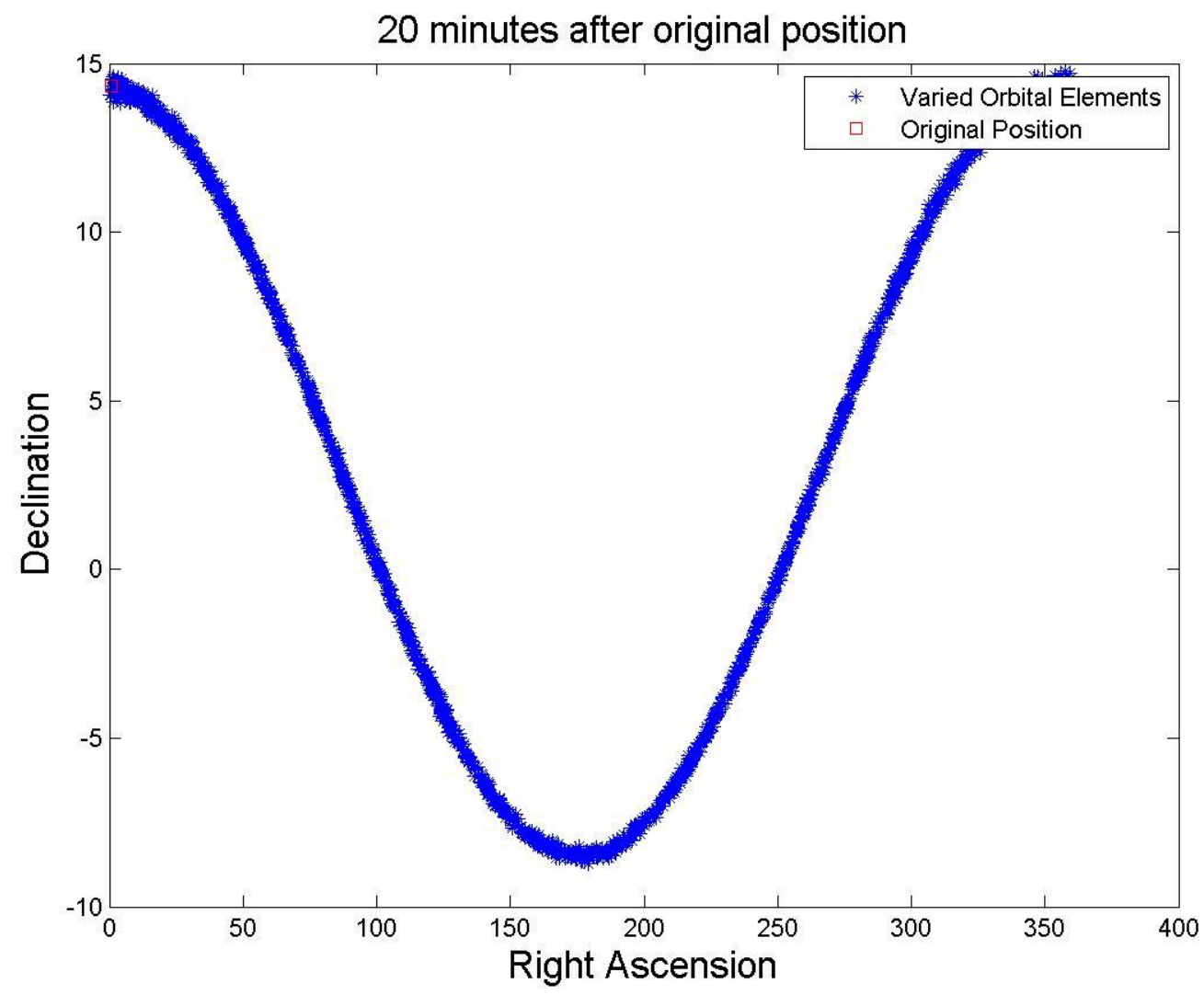



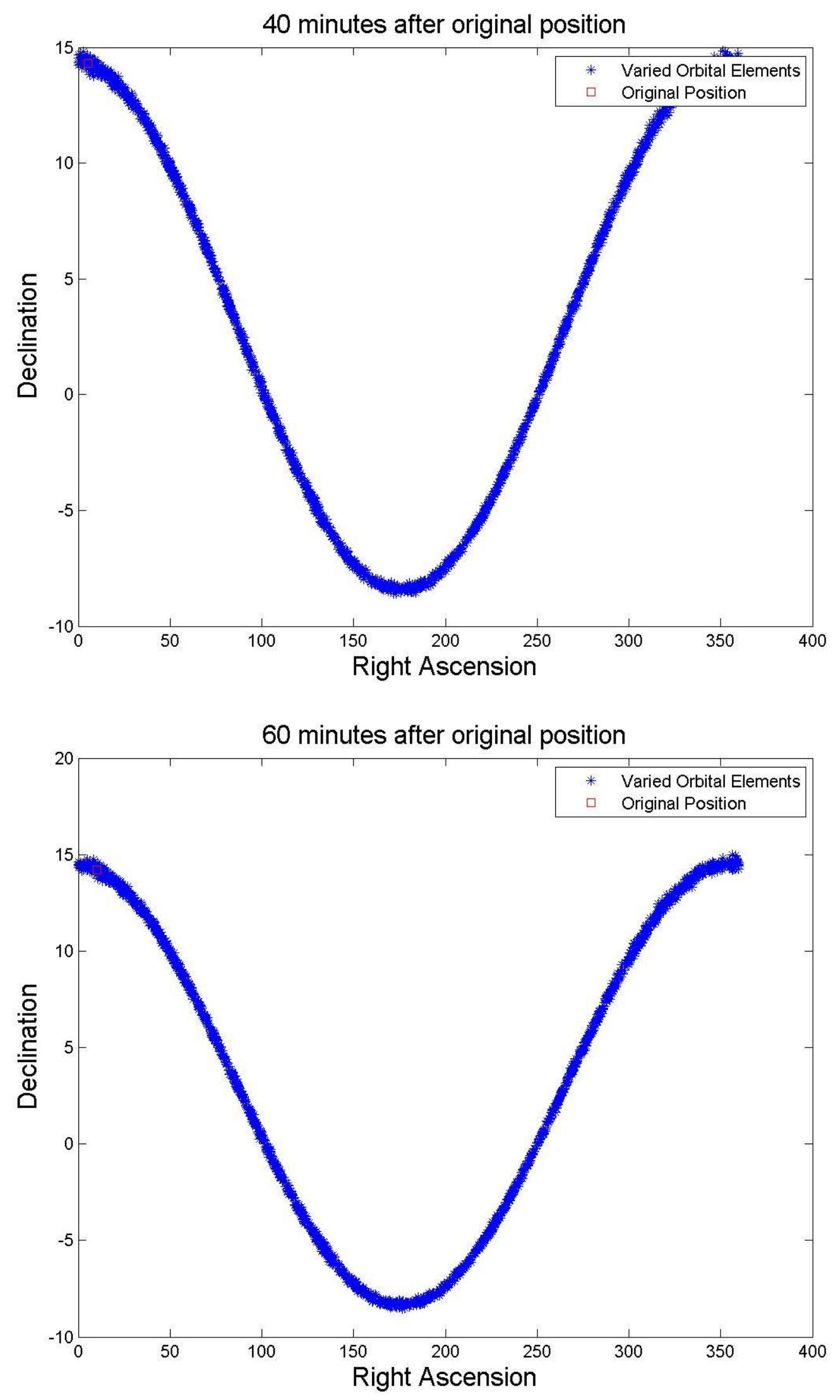


\section{Object 70716}

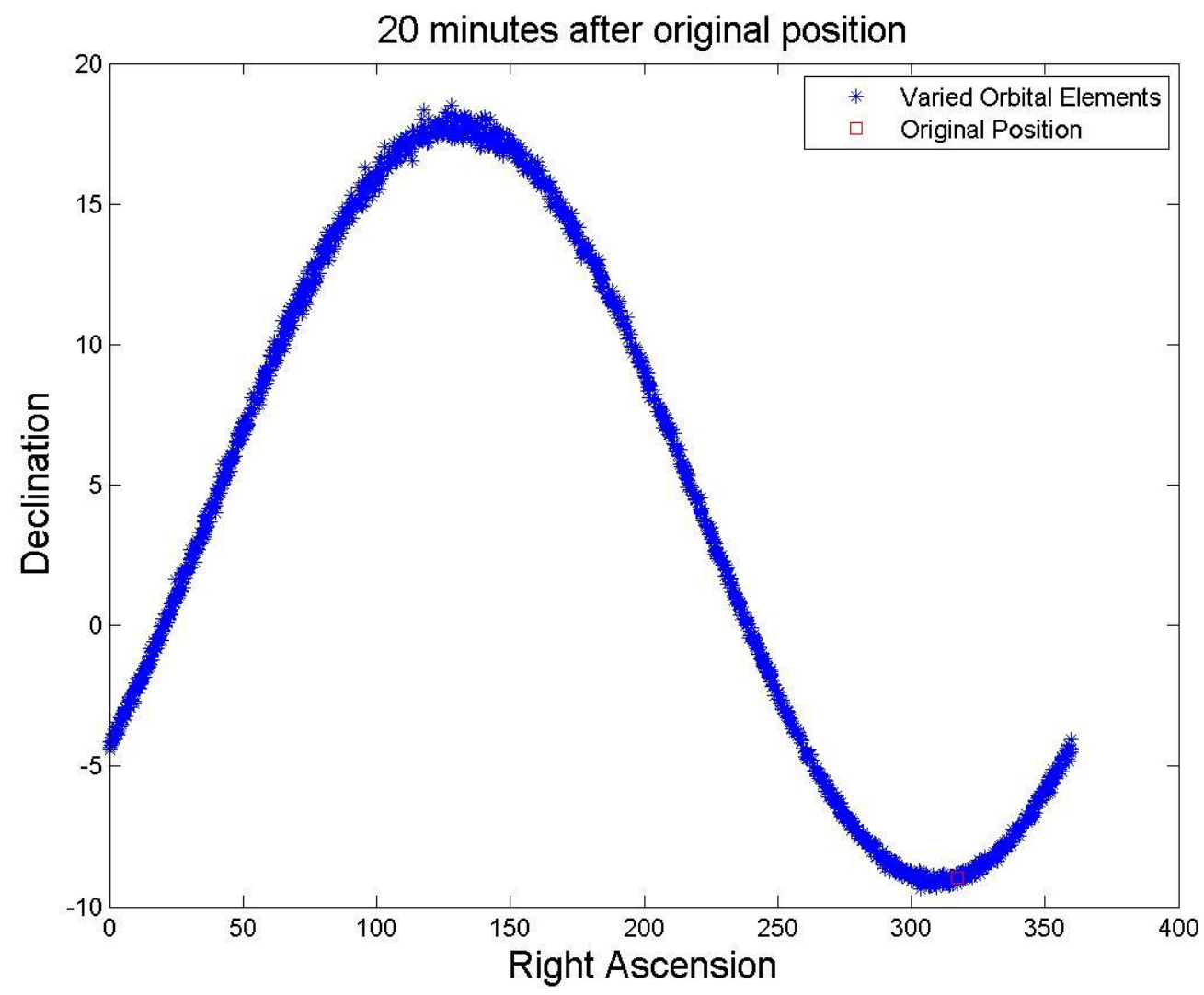



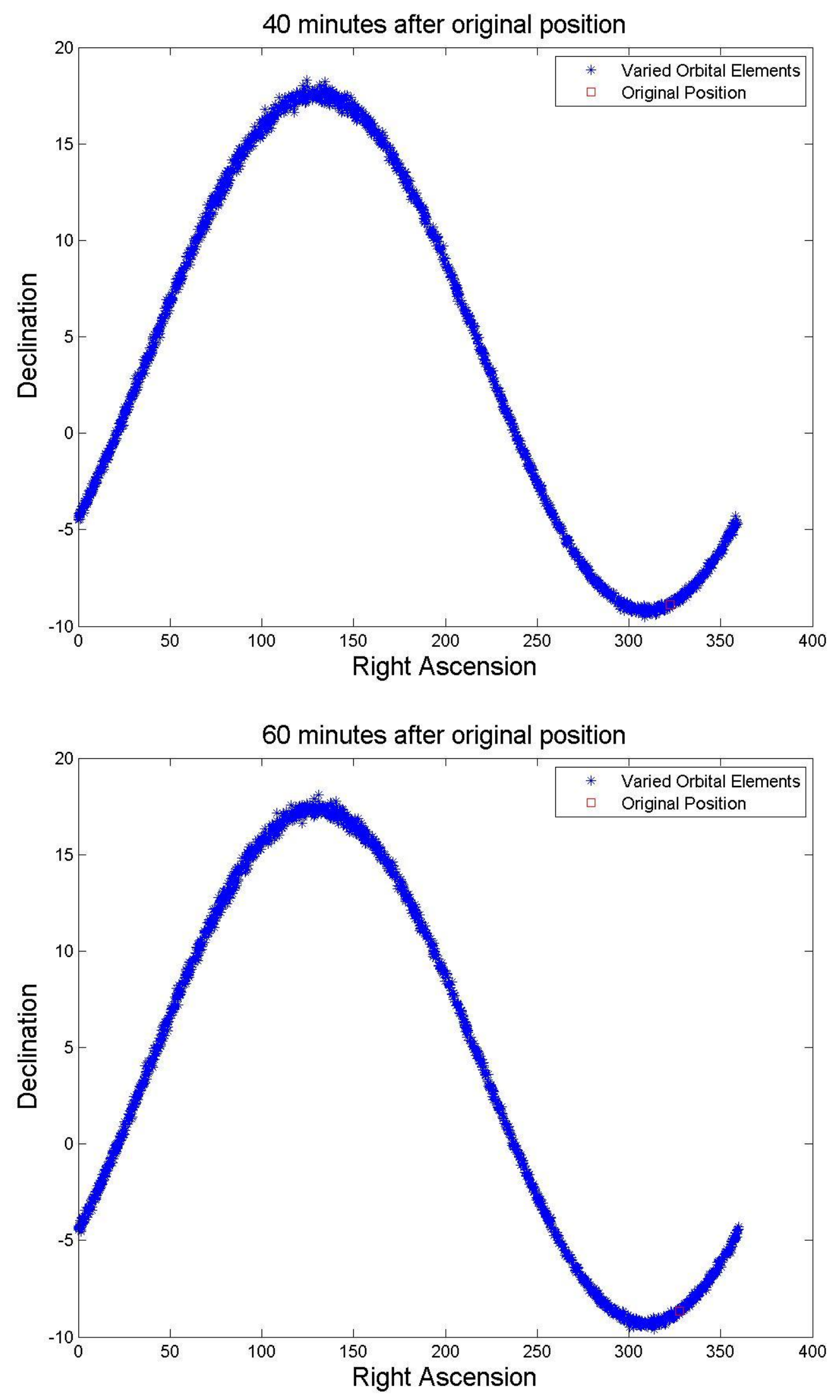